Kristina Peuschel und Jan P. Pietzuch (Hg.)

Kaleidoskop der jungen

DaF-/DaZ-Forschung

Dokumentation zur zweiten Nachwuchstagung des Fachverbandes Deutsch als Fremdsprache
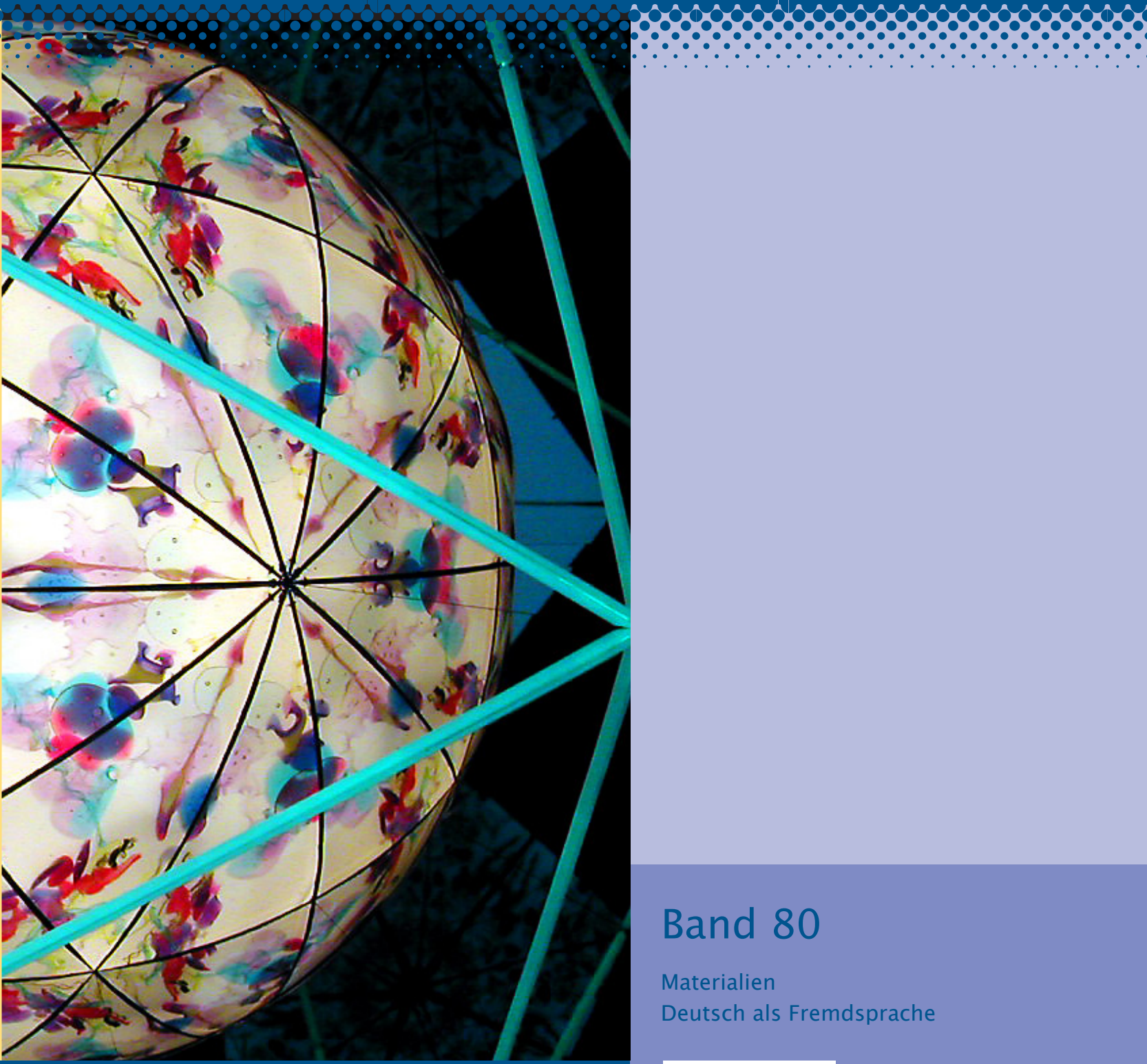

Band 80

Materialien

Deutsch als Fremdsprache

Universitätsdrucke Göttingen

\title{
fadaf
}



Kristina Peuschel und Jan P. Pietzuch (Hg.) Kaleidoskop der jungen DaF-/DaZ-Forschung

This work is licensed under the Creative Commons License 2.0 "by-nd", allowing you to download, distribute and print the document in a few copies for private or educational use, given that the document stays unchanged and the creator is mentioned.

\begin{tabular}{|c|}
\hline C) \\
SOMERTIGHISRESERVED
\end{tabular} 
erschienen als Band 80 in der Reihe „Materialien Deutsch als Fremdsprache” in den Universitätsdrucken im Universitätsverlag Göttingen 2009 
Kristina Peuschel und

Jan P. Pietzuch (Hg.)

\section{Kaleidoskop der jungen DaF-/DaZ-Forschung}

Dokumentation zur zweiten

Nachwuchstagung des Fachverbandes

Deutsch als Fremdsprache

29. und 30. Mai 2007

am Herder-Institut der

Universität Leipzig

Materialien

Deutsch als Fremdsprache

Band 80

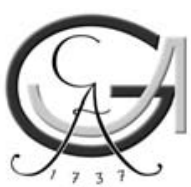

Universitätsverlag Göttingen 2009 


\section{Bibliographische Information der Deutschen Nationalbibliothek}

Die Deutsche Nationalbibliothek verzeichnet diese Publikation in der Deutschen Nationalbibliographie; detaillierte bibliographische Daten sind im Internet über $<$ http://dnb.ddb.de $>$ abrufbar.

Die „Materialien Deutsch als Fremdsprache“ sind eine Reihe des Fachverbands Deutsch als Fremdsprache e.V. $(\mathrm{FaDaF})$, in der Tagungsergebnisse, Dissertationen und andere wichtige Einzeldarstellungen aus dem Bereich Deutsch als Fremdsprache veröffentlicht werden.

http://www.fadaf.de/de/Publikationen/mat_daf/

\section{fadaf}

Dieses Buch ist nach einer Schutzfrist auch als freie Onlineversion über die Homepage des Verlags sowie über den OPAC der Niedersächsischen Staats- und

Universitätsbibliothek (http://www.sub.uni-goettingen.de) erreichbar. Es gelten die Lizenzbestimmungen der Onlineversion. Es ist nicht gestattet, Kopien oder gedruckte Fassungen der freien Onlineversion zu veräußern.

Titelabbildung: Optisches Experiment im Technorama Winterthur;

Fotograf Henning Hraban Ramm/www.pixelio.de

Satz und Layout: Jan P. Pietzuch und Kristina Peuschel

(C) 2009 Universitätsverlag Göttingen

http://univerlag.uni-goettingen.de

ISBN: 978-3-940344-76-2

ISSN: $1866-8283$ 


\section{Inhalt}

\section{Jan P. Pietzuch/Kristina Peuschel (Bielefeld/Leipzig)}

Vorwort: Junge DaF-/DaZ-Forschung zwischen Nachwuchs und Wildwuchs

\section{Uta Großmann (Leipzig)}

Fachtexte richtig lesen - Eine Untersuchung zum inkongruenten

Verstehen ausländischer Studierender bei der Rezeption von

Fachtexten der Wirtschaftswissenschaften. .9

\section{Barbara Haider (Wien)}

„Später dann, wo ich Stärke und Sprache [hatte], dann hab ich nachg'fragt..." - Eine kritische Erhebung sprachlicher Bedürfnisse von nostrifizierten Gesundheits- und Krankenschwestern/-pflegern in Österreich.

\section{Cordula Hunold (Göttingen)}

Kulturspezifische Einflüsse der Lehr- und Lernumgebung auf die (Aussprache)leistungen chinesischer Deutschlernender

\section{Isabelle Maringer (Leipzig)}

Kulturelle Deutungsmuster in deutschsprachigen Medienbeiträgen zum EU-Beitritt der Türkei

\section{Kristina Peuschel (Leipzig)}

Integrierte Textentwicklung in einem Radioprojekt mit fortgeschrittenen DaF-Lernenden.

\section{Jan P. Pietzuch (Bielefeld)}

Kulturelles Lernen als Modifikation personaler Kulturkonstrukte Eine Projektheuristik 


\section{Sabine C. Stricker (Trento)}

Das Europäische Sprachenportfolio zwischen Traum und

Wirklichkeit: Eine kritische Betrachtung dieses Instruments aus der Sicht italienischer LehrerInnen.

\section{Anja Ucharim (Leipzig)}

Die traditionelle Lehrwerkanalyse und die Diskursanalyse -

Zwei Methoden zur inhaltlichen Analyse von Lehrwerken

für Integrationskurse

\section{Paul Voerkel (Belém)}

Kulturwissenschaftliche Diskursanalyse - ein Praxisbericht 169

\section{Daniela Zahn (Glasgow)}

„Der Finger in der Wunde“ - oder einige Anmerkungen zu neurowissenschaftlicher Empirie als Basis didaktisch-methodischer Entscheidungen beim FSL 


\title{
Vorwort: Junge DaF-/DaZ-Forschung zwischen Nachwuchs und Wildwuchs
}

\author{
Jan P. Pietzuch \& Kristina Peuschel
}

Es war zwar neuer Anwuchs hier am Rande des Kulturforstes, aber er hatte Zeit gehabt, wieder mal nachzuwachsen, um die alte Grenze festzuhalten [...]. ${ }^{\mathrm{i}}$

\section{Einleitung}

Der vorliegende Sammelband bietet in Form ausgewählter Beiträge eine kaleidoskopische Dokumentation der zweiten FaDaF-Nachwuchstagung, zu der sich im Mai 2007 mehr als achtzig junge DaF-/DaZ-Nachwuchswissenschaftler/innen in Leipzig versammelten. Diese zweitägige Veranstaltung bot nicht nur Raum zur Präsentation und Diskussion laufender Qualifikationsarbeiten und zur Vernetzung der Teilnehmer/innen (vgl. Punkt 2). Ebenso war es das Anliegen der Tagung, einen intensiv(er)en Austausch über die eigene Verortung des wissenschaftlichen Nachwuchses im DaF-/DaZ-,Kulturforst' anzuregen.

Folgende Spannungsfelder, die mit dem Wortpaar, Nachwuchs und Wildwuchs' bereits angedeutet sind, standen dabei im Vordergrund: Wie systematisch sind die beiden Grundgedanken der Nachwuchsförderung - die Weiterentwicklung des Faches und die Förderung junger Wissenschaftler/innen - miteinander verschränkt? In welchem Verhältnis stehen hierbei auf etablierte Forschungsfelder ausgerichtete Interessen des Faches und neuartige, oft auch unkonventionelle, Erkenntnisinteressen und Ansätze von Qualifikationsprojekten? Wie verorten sich junge DaF-/DaZ-Forscher/innen selbst zwischen ,nachwachsenden' Forschungsfeldern und aktuellen, auch fachübergreifenden Fragestellungen und Forschungsparadigmen? Und schließlich: Wo verläuft die Grenze zwischen einer nachhaltigen, strukturierten Nachwuchsförderung und ggfs. unzureichend ressourcenbewusstem, wissenschaftlichem Wildwuchs?

In der beachtlichen thematischen, theoretischen und method(olog)ischen Diversität der Tagungsbeiträge wurde in diesem Zusammenhang deutlich, dass sich die junge DaF-/DaZ-Forschung immer wieder projektspezifisch zwischen Kontinuität und Innovation - zwischen gepflanztem ,Anwuchs` und kaum steuerbarem ,Wildwuchs ${ }^{\varsigma}$ - positioniert. In den Vorträgen und Posterpräsentationen, aber auch in zahlreichen Gesprächen am Rande zeichnete sich ab, auf welche Weise(n) dabei ,Wildwuchs', der die Grenzen etablierter Forschungsfelder perforiert und ähnlich wie in der Pflanzenwelt durch kaum beeinflussbares Wachstum neue Arten her- 
vorbringt, zur Diversität und Ökologie des Forschungsfeldes DaF/DaZ beitragen kann. Um im Bildbereich zu bleiben: Auch an den hier versammelten Beiträgen ist abzulesen, wie ,Samen' aus verschiedensten, quer zu den Einzeldisziplinen liegenden Theorie- und Forschungsdiskursen in das Feld der Fremdsprachenforschung eingetragen und von jungen Wissenschaftler/innen für $\mathrm{DaF}$-/DaZ-spezifische Fragestellungen, v.a. auch auf methodologisch-empirischer Ebene, fruchtbar gemacht werden.

Gleichsam trat in den Diskussionen zwischen den Teilnehmenden eine stark erhöhte Sensibilität für die Problematik zu Tage, dass ,Wildwuchs', der unkontrolliert wuchert und nur unzureichend gepflegt wird, Gefahr läuft, Früchte minderer Qualität zu tragen und letztlich finanzielle Ressourcen der Nachwuchsförderung ebenso wie persönliche Potentiale des Nachwuchses nicht ausreichend effizient zu nutzen. Insbesondere im Rahmen des Abschlussplenums wurden Aspekte der Qualitätssicherung und -verbesserung in der Nachwuchsförderung, dabei vor allem in der Betreuung von ,freien' Promovierenden, lebhaft diskutiert (vgl. Punkt 3).

In dieser kritisch-konstruktiven Linie ist auch der Titel der vorliegenden Tagungsdokumentation zu verstehen: An die Stelle einer - selbst in Nachwuchspublikationen gelegentlich versuchten - ,Kartografie‘ eines Forschungsfeldes möchten wir die Kaleidoskop-Metapher setzen, um das zwangsläufig Fragmentarische dieses Sammelbandes zu betonen, aber auch - im Sinne des „Schönbildschauers“ (kalós: schön; eĩdos: Gestalt, Bild; skopeĩn: betrachten, schauen ${ }^{\text {ii }}$ - den uns eigenen optimistischen Blick auf die Entwicklung der jungen DaF-/DaZ-Forschung in den Vordergrund zu rücken.

Dem Prinzip der lebendig-bunten Bilderfolge eines Kaleidoskops entsprechend, verzichtet der Sammelband deshalb bewusst auf eine Einordnung der einzelnen Beiträge in bestehende Fachsystematiken und erhebt ebensowenig Anspruch auf Repräsentativität oder die Abbildung eines ,state-of-the-art' neuerer DaF-/DaZ-Forschung.

Insbesondere aber soll das Funktionsprinzip eines Kaleidoskops metaphorisiert und für die vorliegende Publikation fruchtbar gemacht werden: Denn ebenso, wie ein Kaleidoskop meist dreier spezifisch angeordneter Spiegel und kontinuierlicher Bewegung bedarf, um aus bunten Glas- oder Plastikfragmenten im Auge des Betrachters stetig wechselnde geometrische Muster und Bilder zu erzeugen iii, wird sich auch für jede/n Leser/in nur über die mehrfache Spiegelung der hier vorliegenden Texte in den Reflexionsflächen Theorie, Empirie und Praxis sowie deren je spezifische Gewichtung ein kaleidoskopischer Blick auf junge DaF-/DaZ-Forschungsprojekte im „Reifungsprozess“ eröffnen. 


\section{Zur Nachwuchstagung}

Die zweite FaDaF-Nachwuchstagung, die am 29. und 30. Mai 2007 an der Universität Leipzig stattfand, knüpfte an die Initiativen von Jena (2005) und Hannover (2006) an und hatte sich unter dem Motto „Quo Vadis DaF-Nachwuchs“ zum Ziel gesetzt, in einem größeren Rahmen als bisher international verankerte Nachwuchswissenschaftlerinnen und Nachwuchswissenschaftler im Bereich DaF/DaZ zusammen zu bringen. In insgesamt vier Plenarveranstaltungen, fünf Workshops und einer Poster-Session diskutierten junge aktive und zukünftige Wissenschaftler und Wissenschaftlerinnen über 30 Forschungsprojekte aus mehr als zehn Ländern (z.B. Argentinien, Deutschland, Frankreich, Kolumbien, Lettland, Österreich, Polen, Türkei).

Eine Besonderheit der Tagung bestand in ihrer zweifachen Schwerpunktsetzung, die sowohl forschungsthematische als auch forschungsmethodische Tendenzen aufzugreifen beabsichtigte: Während am ersten Tag die Präsentation und Erörterung aktueller Themen und Inhalte von DaF-/DaZ-Qualifikationsprojekten aus den Teildisziplinen Linguistik, Didaktik und Kulturstudien im Vordergrund standen, boten die Plenarsitzungen und Workshops am zweiten Tag Gelegenheit zur Diskussion primär method(olog)ischer Fragestellungen in der Anfangs- und Endphase von Qualifikationsarbeiten. Dieser empirische Fokus ermöglichte jeweils projektspezifische, aber auch vergleichende Diskussionen über Zusammenhänge zwischen Erkenntnisinteressen, theoretischer Positionierung, Forschungsdesigns und Methoden der Datenerhebung und -auswertung, die in ihrem themen- und disziplinübergreifenden Charakter zum Erfolg der Tagung maßgeblich beigetragen haben und im Rahmen der Nachwuchsinitiative(n) unbedingt fortgesetzt und intensiviert werden sollten.

\section{Zur Qualitätssicherung in der Nachwuchsforschung}

Das Anliegen der Tagung, den Dialog über Struktur und Instrumente der Nachwuchsförderung im Bereich DaF/DaZ auf der Ebene junger Forschung selbst zu intensivieren, wurde vor allem in der Abschlussdiskussion aufgegriffen. Auf der Grundlage von Leitfragen, v.a. aber von Erfahrungsberichten der Beitragenden und Expert/inn/en wurden in diesem Plenum Potentiale, Qualität und Grenzen der Betreuung junger Wissenschaftler/innen in Forschung, Lehre und universitärem Alltag kritisch diskutiert und jeweils in Relation zur systematischen Förderung wissenschaftlicher Karrieren einerseits und akademischem Wildwuchs andererseits gestellt. Hierbei verdeutlichten individuelle Qualifikationsbiografien ebenso wie Verweise auf den internationalen Wettbewerb, dass die Themen Qualitätssicherung, Qualitätsstandards und verstärkte Strukturierung der Nachwuchsförderung im Bereich DaF/DaZ einer größeren Aufmerksamkeit und Diskussion bedürfen.

Entsprechend wurde im Abschlussplenum eine „Qualitätsinitiative DoktorandInnenausbildung“"vorgeschlagen, in deren Rahmen fachspezifische, institutions- 
Jan P. Pietzuch \& Kristina Peuschel

übergreifende Leitlinien bzw. Standards für Qualifikationsphasen zu entwickeln sowie unter Berücksichtung der jeweiligen Fachkulturen und Standortspezifika zu implementieren wären. Dabei sollten Erfahrungen aus erfolgreich arbeitenden Graduiertenkollegs sowie Best-Practice-Modelle aus dem internationalen Umfeld nutzbar gemacht werden - vor allem auch für die Betreuung und Förderung des nicht in Forschergruppen eingebundenen oder assoziierten wissenschaftlichen Nachwuchses im klassischen „Meister-Schüler“-Modell.

Als ein konkretes und kostenneutral einsetzbares Instrument wurden Betreuungsverträge diskutiert, die sich in der Nachwuchsförderung anderer Wissenschaftsbereiche bereits etabliert und bewährt haben. Auch in unserem Fach kann das Festhalten von Betreuungsmodalitäten und Anforderungen, die an Promovend/innen und Betreuende im Qualifikationsprozess gestellt werden, zu mehr Transparenz und Orientierung beitragen. Maßnahmen dieser Art können zudem institutionell als Wettbewerbsvorteil verstanden werden und die Qualität der im Fach DaF abzuschließenden Qualifikationsarbeiten sichern helfen (vgl. z.B. die DFG-Rundgespräche zur Förderung wissenschaftlicher Karrieren ${ }^{\text {iv }}$ sowie die DFG-Vorschläge zur Sicherung guter wissenschaftlicher Praxisv).

Aus der aktiven Auseinandersetzung mit der ,Nachwuchs-Wildwuchs-Problematik' resultiert auch die Überlegung, wie fachintern eine stärkere forschungsthematische Vororientierung von DaF/DaZ-Absolvent/innen mit Promotionsabsicht geleistet werden kann und warum eine solche Vororientierung nötig ist. So würden bspw. nach Themenbereichen und Fragekomplexen strukturierte Kataloge eine systematisch(er)e Bearbeitung zentraler Desiderata einzelner Forschungsbereiche ermöglichen und den Interessen des Faches im Bereich der Nachwuchsförderung dienen. Auch auf institutioneller Ebene wären solche Kataloge als Instrumente zur Profilbildung denkbar.

Als qualitätssichernde Maßnahmen können feldspezifische Kataloge von Forschungsdesiderata auf der individuellen Ebene des wissenschaftlichen Nachwuchses erste Weichen stellen und die weiterhin eigenständige Entwicklung einer fachlich-thematisch relevanten Fragestellung unterstützen. Auf diese Weise kann gewährleistet werden, dass der/die junge Forschende an einem (fachlich wie persönlich) ,sinnvollen' Forschungsprojekt arbeitet, das langfristige Motivation sichert, und die Notwendigkeit der eigenen Forschung transparent hält. Zudem können so mehrfache Neu- bzw. Umorientierungen oder gar der Abbruch eines Qualifikationsprojekts vermieden und die Identifikation mit der scientific community intensiviert werden.

Eine solche Verknüpfung von aktuellen Forschungsdesiderata mit eigenen, oft Fachgrenzen überschreitenden, Interessen und Themen des wissenschaftlichen Nachwuchses kann eine fruchtbare Forschungs- und Entwicklungsarbeit im DaF-/ DaZ-Kulturforst ermöglichen. 


\section{Zu den Beiträgen}

Uta Großmann widmet sich in ihrem Beitrag der Rezeption von deutschsprachigen Fachtexten durch ausländische Studierende der Wirtschaftswissenschaften. Dabei werden zunächst Erwartungen an die Fachtextrezeptionskompetenz und die Fachtextverarbeitung durch ausländische Studierende theoretisch herausgearbeitet. Diese Anforderungen bilden die Grundlage der empirischen Untersuchung zum inkongruenten Verstehen bzw. zu Leseverstehensproblemen, deren erste Ergebnisse hier vorgestellt werden.

Barbara Haider geht der Frage nach, welche sprachlichen Bedürfnisse Gesundheits- und Krankenschwestern/-pfleger mit Migrationshintergrund bei ihrem Berufseinstieg in Österreich entwickeln und wie auf diese Bedürfnisse reagiert werden kann. Auf der Grundlage der kritischen Sprachbedarfserhebung (critical needs analysis) werden sowohl die subjektiven Bedürfnisse der Krankenschwestern/-pfleger als auch die objektiven Anforderungen im Beruf (Sprachbedarfe) selbst erhoben sowie die institutionellen Rahmenbedingungen thematisiert. Ziel der Untersuchung ist es, Fördermöglichkeiten für die Betroffenen zu erarbeiten, aber auch nach Veränderungsmöglichkeiten im System zu suchen.

Cordula Hunold hinterfragt die kulturspezifischen Lehr- und Lernweisen chinesischer Deutschlernender beim Erwerb der deutschen Aussprache als Faktorenkomplex von soziopolitischen und soziokulturellen sowie personenbezogenen Faktoren im chinesischen akademischen Kontext. Das Anliegen ihres Beitrags ist es, kulturelle Unterschiede als Einfluss nehmend auf die Ausspracheleistungen chinesischer Studierender darzustellen und so auf empirischer Grundlage zu einer größeren Bewusstmachung für die Problematiken dieses Feldes zu gelangen.

Isabelle Maringer beschreibt die Entwicklung einer Methodik der Datenanalyse, die es ermöglicht, kulturelle Deutungsmuster in öffentlichen Diskursen aufzudecken. Anhand eines Fallbeispiels werden die einzelnen aufeinander folgenden Analysephasen vorgestellt. Als Weiterentwicklung des Deutungsmusteransatzes soll das gewonnene Instrumentarium dazu dienen, dem landeskundlichen Unterricht in Deutsch als Fremdsprache qualitativ neue Impulse zu geben.

Kristina Peuschel stellt eine didaktisch motivierte, explorativ-interpretative Studie vor, die sich dem sprachlichen Lernen in Projekten, speziell in Radioprojekten für DaF/DaZ, widmet. Der Schwerpunkt des Beitrages liegt auf dem Nachvollziehen von Textentwicklungsprozessen, die sich in mündlichen und schriftlichen Teilprodukt(ion)en von Lernenden zeigen. Der Beitrag soll dazu anregen, Projekte als Lehr-Lernform zu entmystifizieren, sich den Potentialen und Grenzen der Förderung sprachlichen Lernens in Projekten zuzuwenden und dahingehend weitere empirische Untersuchungen durchzuführen.

Jan Paul Pietzuch skizziert die heuristischen Grundlagen und das Forschungsdesign einer empirischen Langzeitstudie, die individuelle kulturelle Lernprozesse im Kontext des Fremdsprachenerwerbs als Modifikation personaler Kulturkonstrukte 
Jan P. Pietzuch \& Kristina Peuschel

untersucht. Dazu schlägt er eine funktional auf das empirische Design bezogene Refiguration der drei Kernkonzepte ,Kultur', ,Subjekt' und ,Lernen“ mit Hilfe der analytischen Kategorien a) Diskursivität, b) Narrativität und c) Dialogizität vor. Das daraus entwickelte heuristische Modell zum kulturellen Lernen, dessen method(olog)ische Implikationen sowie das Konzept der ,personalen Kulturkonstrukte sollen es ermöglichen, kulturelle Lernprozesse als Modifikation individuell verfügbarer Schematarepertoires zu erforschen.

Sabine Stricker beschreibt Diskrepanzen zwischen Anspruch und Realität, die seit der Einführung des Europäischen Sprachenportfolios (ESP) in Italiens Schulen im Jahr 2003 aufgetreten sind. In ihrem Beitrag werden die theoretischen Konzepte und Ziele des ESP den Erwartungen, Bedenken und Fragen italienischer DaFLehrerInnen gegenübergestellt.

Anja Ucharim geht es um den Zusammenhang von Wirklichkeitskonstruktion, Integrationskonzept und pädagogisch-landeskundedidaktischen Ansätzen in Lehrwerken für Integrationskurse in der Bundesrepublik. Im Zentrum ihrer als thematische Diskursanalyse angelegten Lehrwerkanalyse stehen die sprachlichen und visuellen Mittel, mit deren Hilfe Wirklichkeit konstituiert wird. Neben der Projektskizze und der Präsentation der Forschungsmethodik werden erste Beispielanalysen und Ergebnisse dargestellt.

Paul Voerkels Beitrag fasst die Ergebnisse einer kulturellen Diskursanalyse, die er im Rahmen seiner Magisterarbeit durchführte, am Beispiel Fußball zusammen. Er beschreibt vorwiegend Erfahrungen mit dem Verfahren der kulturellen Diskursanalyse und reflektiert die Frage, inwieweit mit der Beschreibung von ,kulturellen Deutungsmustern' das kulturelle Lernen im Fremdsprachenunterricht, insbesondere in Deutsch als Fremdsprache (DaF), gefördert werden kann.

Daniela Zahn untersucht am Beispiel prosodischer L2 Forschung Möglichkeiten der Übertragung von Ansätzen der neurowissenschaftlichen Empirie auf didaktisch-methodisches Handeln. Trotz der unterschiedlichen Gegenstände beider Disziplinen wird diskutiert im Interesse der Entwicklung einer soliden und kohärenten Fremdsprachenlerntheorie auf Ergebnisse der neurowissenschaftlichen Sprachlernforschung zurückgegriffen, um didaktisch-methodisches Vorgehen empirisch zu untermauern.

\section{Dank}

Wir freuen uns, dass der FaDaF die Initiative des fachlichen Nachwuchses fördert und die Möglichkeit der Veröffentlichung der Beiträge in der Reihe Materialien DaF unterstützt. Dafür möchten wir uns herzlich bedanken. Ebenso möchten wir uns ganz herzlich für die Unterstützung bei der Durchführung der Tagung beim FaDaF, dem Hueber-Verlag, dem Herder-Institut und der Universität Leipzig bedanken. Unser besonderer Dank gilt auch Frau Prof. Aguado, Herrn Prof. Ahren- 
holz, Frau Dr. Basteck, Frau Dr. Demmig, Herrn Prof. Fandrych, Frau Prof. Mehlhorn, Frau PD Dr. Reinke, Herrn Prof. Schmelter, Frau Prof. Schramm, Herrn Prof. Tschirner und Herrn Dr. Wormer, die die Diskussionen in den Sektionen geleitet haben und mit zahlreichen Hinweisen und Anregungen auch zur Auswahl der Beiträge für diesen Band beigetragen haben. Wir möchten uns auch bei den Studierenden des Herder-Instituts der Universität Leipzig bedanken und natürlich in ganz besonderem Maße bei den Referentinnen und Referenten und den interessierten Zuhörenden, die zum Gelingen der Tagung beigetragen haben.

Bielefeld \& Leipzig, Oktober 2008

Jan P. Pietzuch \& Kristina Peuschel

i Raabe, Wilhelm (1911): Altershausen. Berlin: Jahnke, 268. (entnommen aus dem DWDS-Kernkorpus/Textbeispiele für, nachwachsen').

ii http://www.wdrmaus.de/sachgeschichten/kaleidoskop [22.09.2008].

iii Duden (2001): Deutsches Universalwörterbuch, 4. Auflage. Mannheim: Duden-Verlag, 866.

iv http://www.dfg.de/dfg_im_profil/aufgaben/nachwuchsfoerderung/aufgaben_nachwuchsfoerderung_dokumente.html [22.09.2008].

v Deutsche Forschungsgemeinschaft (1998): Vorschläge zur Sicherung guter wissenschaftlicher Praxis: Empfehlungen der Kommission „Selbstkontrolle in der Wissenschaft". Weinheim: Wiley-VCH. [http:// www.dfg.de/aktuelles_presse/reden_stellungnahmen/download/empfehlung_wiss_praxis_0198. pdf; 22.09.2008]. 



\title{
„Fachtexte richtig lesen“ -
}

\author{
Eine Untersuchung zum inkongruenten Verstehen ausländischer \\ Studierender bei der Rezeption von Fachtexten der Wirtschafts- \\ wissenschaften
}

\section{Uta Großmann (Leiprig)}

\begin{abstract}
Dieser Beitrag orientiert sich an meinem Dissertationsprojekt zur Aufnahme von inkongruentem Verstehen bei der Rezeption von Fachtexten ausländischer Studierender der Wirtschaftswissenschaften. Zunächst wird in einer Einführung das Anliegen und Erkenntnisinteresse der Dissertation vorgestellt. Der zweite Abschnitt erörtert theoretisch fundiert, welche Fachtextrezeptionskompetenzen erwartet werden. Dabei stehen zentrale Definitionen im Mittelpunkt, die hinsichtlich der Anforderungen an die Fachtextverarbeitung durch ausländische Studierende ausgelegt werden. Diese Anforderungen bilden die Grundlage der empirischen Arbeit, die hier beispielhaft in Abschnitt drei dargestellt wird. Ausgehend vom Forschungsansatz präsentiert dieser Beitrag eine Möglichkeit der Dateninterpretation zur Ermittlung von inkongruentem Verstehen (Leseverstehensproblemen). Abschließend werden die ersten Ergebnisse hinsichtlich ihrer Relevanz zum Erkenntnisinteresse diskutiert.
\end{abstract}

\section{Einleitung}

Das Lesen ist eine „[...] heute in unserer Kultur [...] täglich benötigte Fähigkeit“ (Grzesik 1990: 9). Dabei bestimmt der Leser selbst die Ausprägung dieser Fähigkeit, da sie „[...] unabhängig vom jeweiligen Entwicklungsstand des einzelnen“ (ebd.) ist und von Individuum zu Individuum je nach Lesesituation variiert. Allgemein lässt sich das Lesen als aktiver Prozess der Bedeutungskonstruktion beschreiben (vgl. Schramm 2001: 50; Christmann \& Groeben 1996: 1536), bei dem

die Rezipienten auf der Grundlage ihrer inhaltlichen Vorkenntnisse und ihres enzyklopädischen Wissens die Textinformation aktiv-konstruktiv in ihre Wissensstruktur einfügen (Christmann \& Groeben 1996: 1536).

Dies kennzeichnet den Verstehensprozess des Studierenden als einen individuellen, der auf einer Interaktion zwischen dem kognitiven Wissen des lesenden Studierenden und dem zu lesenden Text beruht. Dabei konstruiert der Leser eine mentale Repräsentation des im Text dargestellten Sachverhaltes. Das Ergebnis der mentalen Repräsentationsleistung ist im Studium von größter Bedeutung, da nur durch textnahe Verarbeitung der Fachlektüre studienrelevante Aufgaben qualitativ hoch- 
wertig erarbeitet werden können. Dies ist entscheidend für die Qualität des Studienabschlusses, da ein Studium an deutschen Universitäten zum großen Teil aus der Rezeption von Fachtexten besteht. Diese Fähigkeit, die im Rahmen der Umstellung auf Bachelor- und Masterstudiengänge (Bologna-Ergebnis) noch verstärkt gefordert wird, da in verkürzter Studienzeit große Mengen an Wissen aufgebaut werden müssen, erfordert eine gründliche Reflexion der Leseleistungen aller Studierenden. Diese Arbeit möchte sich einem Teil der Studierenden widmen, den der ausländischen Studierenden. Damit reflektiert diese Arbeit den aktuellen Stand der Immatrikulationen der meisten deutschen Universitäten. ${ }^{i}$ Dieser immense Zuspruch, der den deutschen Universitäten widerfährt, erfordert auch eine sprachliche Integration der ausländischen Studierenden. Es gilt zwar für diese, dass Erstsemesterimmatrikulierte ein Sprachdiplom vorweisen müssen, meist die DSHPrüfung Stufe 1, doch handelt es sich hierbei um ein Zertifikat, bei dem die Studierenden in Sprachschulen oder in Eigeninitiative direkt auf die Prüfungssituation vorbereitet werden, die einem Sprachvermögenstest entspricht. Die Leseleistung wird dabei nur geringfügig getestet, vor allem aber nicht die fachsprachbezogene Textrezeption. Im Fachstudium werden jedoch neben allgemeinen Leseverstehensleistungen vor allem die fachsprachlichen Rezeptionskompetenzen gefordert. Dieser Forderung soll im Rahmen des Dissertationsprojektes nachgegangen werden.

Das Ziel der Dissertation ist es, die auftretenden Leseschwierigkeiten bei der Fachtextrezeption ausländischer Studierender der Wirtschaftswissenschaften zu erfassen, qualitative Häufigkeitsmerkmale des inkongruenten Verstehens zu beschreiben und didaktische Implikationen und Handlungsempfehlungen abzuleiten. Dabei wird folgendermaßen vorgegangen: Zuerst sollen die in der aktuellen Forschungslage zur Rezeption von (Fach)Texten problematisierten Leseverstehensschwierigkeiten systematisiert werden. Anschließend werden die empirisch erfassten Datenreihen (Ist-Zustand) ausgewertet und aufgetretene „Lesefehler" (inkongruentes Verstehen nach Schramm 2001)ii ermittelt. Im Anschluss daran werden diese Daten miteinander verglichen und die Ergebnisse kategorisiert. Das Zielprodukt stellt ein Katalog von inkongruentem Leseverstehen ausländischer Studierender dar, der (möglicherweise) Handlungsanweisungen an Dozenten aber auch Studierende enthält, wie den häufigsten Fehlern entgegengewirkt werden kann.

Im Rahmen dieses Artikels werden die Fachtextrezeptionsprobleme, die der aktuellen Forschungslage zu entnehmen sind, aufgeführt, diskutiert und als Grundlage der weiteren empirischen Forschungsarbeit dieser Dissertation zur Diskussion gestellt. Der Artikel orientiert sich an folgenden textgliedernden Fragen:

- Welche Fachtextrezeptionskompetenzen werden erwartet, um Fachtexte lesen zu können?

- Wie können die Fachtextrezeptionsprobleme der ausländischen Studierenden im Rahmen der Dissertation nachgewiesen werden?

- Welche Relevanz haben die vorläufigen Untersuchungsergebnisse? 


\section{Welche Fachtextrezeptionskompetenzen werden erwartet, um Fachtexte lesen zu können?}

Einen Fachtext aufgabengemäß und gegenstandsadäquat zu rezipieren, erfordert eine Vielzahl von Teilkompetenzen: So muss der Leser über sprachliches (semantische, syntaktische, lexikalische) Wissen verfügen, aber auch über Fertigkeiten, die fachkommunikativ und textorientierend sowie lesestrategisch einen Zugang zum Fachtext ermöglichen. Da in dem Dissertationsprojekt ausländische Studierende der Wirtschaftswissenschaften im Mittelpunkt stehen, sollen diesbezüglich textlinguistische und -pragmatische Zusammenhänge für die Fachsprache Wirtschaftsdeutsch betrachtet werden.

Das Lesen eines Fachtextes gilt als fachkommunikative Handlung, bei der ausgehend von einem schriftsprachigen Text der Rezipient mit dem Autor des Textes kommuniziert. Im Fall des inkongruenten Verstehens erfolgt die Betrachtung der Autor-Text-Interaktion in Anlehnung an die zerdehnte Sprechsituation (Ehlich 1984). Kongruentes Verstehen basiert auf der Übernahme der Wissensvermittlungsabsicht des Autors durch den Leser und des damit verbundenen leserseitigen Wissensaufbaus. Inkongruentes Verstehen beschreibt das Gegenteil, wenn die vom Autor antizipierten Wissenslücken der Leser von ihnen nicht erkannt und „gefüllt“ werden können, sei es aus Gründen einer anderen Textinformationsaufnahmeabsicht oder sei es aus sprachlichen Gründen (funktionale und inhaltliche Inkongruenz). Ausgangsbasis dieser Interaktion ist der Text, der gekennzeichnet ist durch eine begrenzte Folge sprachlicher Zeichen, die kohärent und als Ganzes eine erkennbare kommunikative Funktion signalisieren (vgl. Brinker 2005: 17). Die kommunikative Funktion bestimmt sich aus der Handlungsabsicht des Autors, neues Wissen zu vermitteln und dem damit verbundenen Wissensaufbau des Lesers. Der Autor ist dabei an eine Vielzahl textueller Merkmale gebunden. Zunächst bedarf der Autorentext eines situativen Kontextes, dessen Merkmale durch Textthematik, Handlungsaufbau/-struktur und Textform gekennzeichnet sind und sich in einer Texttypologie widerspiegeln. Der situative Kontext legt aber auch fest, in welcher Form der Text präsentiert werden muss. Dies entspricht einer Einteilung in Klassen von Texten (vgl. Satzger 1993: 301), den so genannten Textsorten (vgl. Gläser 1990: 29; 1993: 20). Sie verbinden kontextuelle (situative), kommunikativfunktionale und strukturelle (grammatische und thematische) Merkmale (vgl. Brinker 2005: 144) mit dem Ziel, Handlungs- und Bewertungsnormen zu vermitteln. Diesbezüglich werden Textsorten nach ihrer Zugehörigkeit zu spezifischen Kommunikationsbereichen klassifiziert, die sich als offiziell vs. inoffiziell, beruflich/institutionell vs. privat charakterisieren lassen (vgl. Brinker 2005: 136). Der hier untersuchte Kommunikationsbereich entspricht einem institutionellen, dem einer Hochschule, in dem ,typische Ziele/Zwecke von den in charakteristischer Weise Handelnden mit Hilfe typischer Handlungen und Sprachhandlungen verfolgt werden“ (Heinemann 2000: 702). Das dabei ,typische“ Verhalten kann als Handlung im 
Fach verstanden werden, wobei sich entsprechend die Kommunikation durch Fachtexte vollzieht. Fachtexte sind dabei

[...] Mittel und Ergebnis der kommunikativen Tätigkeit über fachliche Gegenstände. Sie sind immer in eine konkrete Kommunikationssituation eingebettet, die durch den Kommunikationsbereich (die Domäne), durch die Kommunikationspartner, ihre soziale und fachliche Stellung zueinander sowie ihre Fachkompetenz, durch das Medium der Kommunikation und die Kommunikationsintention determiniert werden (Hoffmann 1988: 53).

Für die Kommunikation im Fach im Bereich der Hochschule auf der Basis des Fachtextes haben sich konventionalisierte, funktionale Darstellungsarten (vgl. Schnotz 1996: 972) herausgebildet. Dabei erfolgt die Realisierung der textuellen Mitteilungsintentionen im Hochschulbetrieb vor allem aber nicht ausschließlich in den Fachtextsorten (siehe Gläser 1990: 29, 1993: 20) des Lehrbuchtextes und des Zeitschriftenartikels. Die Fähigkeit, die verschiedenen Fachtextsorten zu erkennen und ihre inhaltlichen Präsentationen zu erfassen, stellt die erste Kompetenz dar, die ein ausländischer Studierender besitzen muss, wenn er sich deutschen Fachtexten nähert. Dabei soll hier auf die Forschung von Gläser (1990) verwiesen werden, die englischsprachige Text analysiert, kategorisiert und funktional entschlüsselt hat. Ihre dabei erarbeiteten Inhaltskataloge der Fachtextsorten können zur Charakterisierung deutscher Fachtexte als unterstützend übernommen werden. Das Wissen um die Unterscheidung von Fachtextsorten und ihrer inhaltlichen Darbietung allein reicht jedoch nicht, um einen Fachtext kompetent rezipieren zu können. Ausländische Studierende haben zumeist in ihren Heimatländern ein Studium begonnen oder abgeschlossen, bevor sie nach Deutschland kommen, daher besitzen sie die so genannte Fachkenntnis. Problematisch an dieser Situation ist jedoch, dass die Vermittlung von vertiefendem Fachwissen in deutschen Fachtexten nur über eine entsprechende Fachsprache ,Deutsch' möglich ist.

Die Fachsprache Wirtschaftsdeutsch ist von der Wirtschaftslinguistik geprägt, die sich als Teilbereich der Fachsprachen- bzw. Fachkommunikationsforschung präsentiert.iii Bezüglich der Definition der Fachsprache Wirtschaft liegt im gesamten Forschungsbereich der Wirtschaftslinguistik noch kein schlüssiger Kriterienkatalog vor, welcher von der Komplexität und Vielschichtigkeit des Begriffes zeugt.iv Die Sprache der Wirtschaft schließt sowohl Wirtschaftspolitik, Börsensprache aber auch den konsumorientierten Sprachgebrauch in Industrie und Handel sowie studienspezifische Teilbereiche der Wirtschaft (wie Betriebswirtschaftslehre, Volkswirtschaftslehre, Internationale Beziehungen usw.) ein. Diese Heterogenität in der Sprachanwendung, zeigt das weite Spektrum auf, welches von der Fachsprachenlinguistik zu erforschen, zu analysieren und vor allem einzugrenzen ist (vgl. Höhne 1991: 434).

Einen Versuch zur Eingrenzung der Fachsprache Wirtschaftsdeutsch unternehmen Buhlmann \& Fearns (2000: 307) mit ihrer Einteilung, die Fachsprache Wirtschaft vier Kommunikationsbereichen zuzuordnen: Kommunikation von 
Betrieb zu Betrieb, Kommunikation im Betrieb, Allgemeine Information (Lexikon, Handbuch, etc.) und aktuelle Information (Zeitung, Zeitschrift, etc.). Diese Einteilung mag eine erste Abgrenzung darstellen, jedoch erscheint sie mir viel zu allgemeinsprachenorientiert und wenig nützlich, hieraus eine allgemeingültige Definition für die Wirtschaftsfachsprache abzuleiten.

Auf der Suche nach eben dieser allgemeingültigen Definition für die Fachsprache Wirtschaft, sind weit und enger gefasste Definitionsansätze zu ermitteln. Eine sehr weit gefasste Definition geben Buhlmann \& Fearns in einer Abwandlung der Hoffmannschen Fachsprachendefinition:

Wirtschaftssprache, das ist die Gesamtheit aller Fachsprachen, d.h. aller sprachlichen Mittel, die in einem fachlich begrenzten Kommunikationsbereich, nämlich dem der Wirtschaft, verwendet werden, um die Verständigung der in diesem Bereich tätigen Menschen zu gewährleisten (2000: 306).

Diese Definition zeigt auch das Dilemma, in dem sich die Wirtschaftslinguistik befindet: Wirtschaftsdeutsch wird als Sammelbegriff für diverse Fachsprachen innerhalb der Wirtschaft benutzt (vgl. Heuberger 1997: 58). Die Existenz der unterschiedlichen Wirtschaftssprachen ist eine Folge der ,arbeitsteiligen Differenzierung und Spezialisierung der industriellen Wirtschaftsgemeinschaft" (Bungarten 1988: 19f.) und wird mit den Worten Ohnackers (1992: 36) als „Summe der Fachsprachen $[\ldots]$, die in der wirtschaftsbedingten Kommunikation benutzt werden“, zusammengefasst.

Dazu stellt Bolten eine Antithese auf, indem er nachvollziehbar behauptet, dass

[...] das Sprachgeschehen im Wirtschaftsbereich nicht additiv sondern integrativ beschreibbar ist als ein komplexes, empirisch annähernd bestimmbares Beziehungsgeflecht verschiedener fach- und (im weitesten Sinne) berufssprachlicher Ebenen (1991: 74).

Bolten schlägt vor, am Begriff Wirtschaftsdeutsch festzuhalten:

Abgesehen von der Ein- und Ausgrenzbarkeit der einzelnen Subsysteme lassen sich die jeweiligen Beschreibungsergebnisse natürlich nicht zu einem übergeordneten Sprachsystem, Wirtschaftsdeutsch' zusammenfügen. [...] Sprachliche Handlungsfähigkeit, sei es im deutschen Wirtschaftsalltag selbst oder aber im Außenkontakt mit deutschen Unternehmen, lässt sich sicherlich nicht durch die Kenntnis einer oder mehrerer Subsprachen [...] erreichen (ebd.: 73).

Für das Dissertationsprojekt ist daher der Schluss zu ziehen, dass die Fachsprache Wirtschaftsdeutsch nicht als selbständige Erscheinungsform existiert, sondern eine Gesamtheit der sprachlichen Mittel auf der Wort-Satz-Ebene darstellt und damit stets mündlich oder schriftlich aktualisiert wird. Dies begründet sich $\mathrm{m}$. E. darin, dass eine die Teilbereiche der Wirtschaft übergreifende Verwendung der Lexik vorliegt. Beispielhaft demonstriert der Terminus „Wertsteigerung“ eine bedeutungsgleiche Definition in den unterschiedlichen Teilbereichen der Fachsprache 
Wirtschaftsdeutsch der Börse, des Managements, der Investitionen, des Bank- und Rechnungswesens, etc. Er kennzeichnet Maßnahmen, die eine Gewinnmaximierung zur Folge haben. Aus diesem Grund ist es sehr schwierig, Fachsprachen als Subsprachen für den diversen Fachbereich zu differenzieren, welches eine generelle Einteilung in drei Klassen rechtfertigt (vgl. Blei 1993: 398):

- sprachliche Mittel, die in allen Subsprachen auftreten;

- sprachliche Mittel, die in allen Fachsprachen auftreten;

- sprachliche Mittel, die nur in einer Fachsprache auftreten.

Eine Zuwendung von verallgemeinernden Mitteln der Fachsprachenbeschreibung ist insofern gerechtfertigt, als im Mittelpunkt der Untersuchung Texte stehen, ,[...] deren Funktion es ist, als Ensemble verschiedener morphologischer, semantischer und syntaktischer Konstituenten bzw. textorganisierender Prinzipien die Fachkommunikation möglichst umfassend zu gewährleisten“ (Baumann 1995: 333). Eine zu starke Differenzierung der Fachsprachen verwirrt mehr das Textverstehen, als Nutzen gewonnen werden kann. Denn in jedem Fall wird die fachliche Vorbildung als eine feststehende Größe für den Fachspracheneinsatz (vgl. Steinmetz 2000: 146) vorausgesetzt, die eine Fachkraft vom Laien unterscheidet.

Das Dissertationsprojekt untersucht die Kommunikation im Fachbereich Wirtschaft zwischen ausländischen Fachstudierenden und den, vom Autor vermittelten Informationen im Fachtext. Der Leserezeptionsprozess wird dabei untersucht hinsichtlich der beim Studierenden stattgefundenen Verarbeitung der autorenseitig bestimmten Wissenslücke. Wobei kongruentes Verstehen vorausgesetzt wird, wenn der ausländische Studierende eine äquivalente Lösung anbietet. Viel wichtiger jedoch für den Untersuchungsansatz dieses Dissertationsprojektes ist die Aufnahme von inkongruentem Verstehen, den sogenannten Leseproblemen. Im Rahmen der Fachsprache Wirtschaftsdeutsch ergeben sich diese vor allem aus der Problematik der Fachsprache (Fachlexik) und ihrer Verarbeitung in Textform (Mikro- und Makrostruktur von Texten, Textsorteneinteilung, etc.). Um Fachtexte richtig lesen zu können, müssen ausländische Studierende in ihren Kompetenzen gestärkt werden, die sich besonders auf die Verarbeitung von Fachsprache hinsichtlich der (1.) Trennung von Fach- und Allgemeinwörtern und (2.) der Vereinfachung durch Terminologie- und Nomenklaturordnungen beziehen aber auch auf (3.) die Kenntnis der Fachtextstruktur.

(1.) Grundlage bildet das Wissen um die Fachsprache, ,[...] eigene Struktur- und Gliederungsprinzipien, die in der Gemeinsprache, zumindest in dieser differenzierten Form, nicht vorkommen“ (Morgenroth 1993: 47) besitzt. Verallgemeinernd lässt sich feststellen, „dass nur bestimmte Gruppen semantischer und syntaktischer Stilfiguren im Fachtext vorkommen und dass sie überwiegend dem logischen Prinzip der Informationsvermittlung [...] dienen“ (Gläser 1978: 164). Aufkommende Verständnisprobleme lassen sich eingrenzen, indem man Wortschatzstrukturen der Allgemeinsprache von der Fachsprache Wirtschaft trennt. Nach Ihle-Schmidt 
(1983) lässt sich die Wortschatzstruktur der Wirtschaftsprache in fünf lexikalische Kategorien untergliedern:

- Strukturwörter: nach, für, zwei, sein, haben etc.

- Wörter der Gemeinsprache: verstehen, gut, Wort, Haus etc.

- Allgemeiner wissenschaftlicher Wortschatz: Reduktion, abstrakt, Hochrechnung, etc.

- Allgemeiner wirtschaftlicher Wortschatz: Arbeitslosigkeit, Wachstum, Inflation etc.

- Wirtschaftlicher Wortschatz: Außenfinanzierung, Investitionsrücklage, Cash flow.

Eine derartige Einteilung der Wortschatzstruktur stellt eine Möglichkeit dar, die Fachtextrezeptionskompetenz ausländischer Studierender zu stärken. Der Vorteil besteht darin, dass sich die ausländischen Studierenden nicht einer Unmenge neu zu lernender Wörter gegenübersehen, sondern nur einem begrenzten Rahmen, dem des wirtschaftlichen Wortschatzes. Der größere Umfang der Kategorien ist den Studierenden im Rahmen ihrer Allgemeinsprachenausbildung vermittelt worden, darunter verstehe ich das Erlernen von Strukturwörtern, Internationalismen, aber auch den allgemeinen wissenschaftlichen/wirtschaftlichen Basiswortschatz. Der Unterschied, der zwischen allgemein wirtschaftlichem und wirtschaftlichem Wortschatz existiert, lässt sich damit beschreiben, dass es Wörter gibt, die sowohl bezeichnend für die Nutzung in der Allgemeinsprache sind, aber gleichzeitig auch eine fachsprachliche Bedeutung implizieren. So kann das Wort „Wachstum“ allgemeinsprachlich u.a. den Prozess des Wachsens von Pflanzen, Tieren, Menschen beschreiben. Im wirtschaftswissenschaftlichen Bereich kennzeichnet das Fachwort „Wachstum“ u.a. die Anhäufung von Kapital, von Börsenwerten, etc. Das Wissen um die Mehrfachbedeutung kann positiv hinsichtlich der Unterscheidung/Verwendung der Fachlexik angesehen werden. Negative Auswirkungen zeigen sich bezüglich der Mehrfachbedeutung von Wörtern und ihres differenzierten Einsatzes.

(2.) Auch hierzu bietet die Forschung bereits einen Lösungsansatz, wie die Fachtermini erfasst werden können: Die semantisch-logische Gliederung mittels Terminologie- und Nomenklaturzuordnungen. „Die Terminologie einer Fachsprache ist das gedanklich-systematische Gerüst der Wissenschaft, während Nomenklaturen wie bloße empirische Listen bestimmten Einheiten der Terminologie zugeordnet werden" (Morgenroth 1993: 56; Herv. i.O.). Die Terminologisierung versteht sich hier als Systematisierung (Oberbegriffe), wobei sich der Leser bereits aus dem deklarativen Wissensbeständen das Wort erschließen kann, während Nomenklatur als Klassifikation (Unterbegriffe) verstanden wird (vgl. Abbildung 1), deren Bedeutung sich der Lesende aus dem Kontext mittels zusätzlicher Informationen erarbeiten muss. 


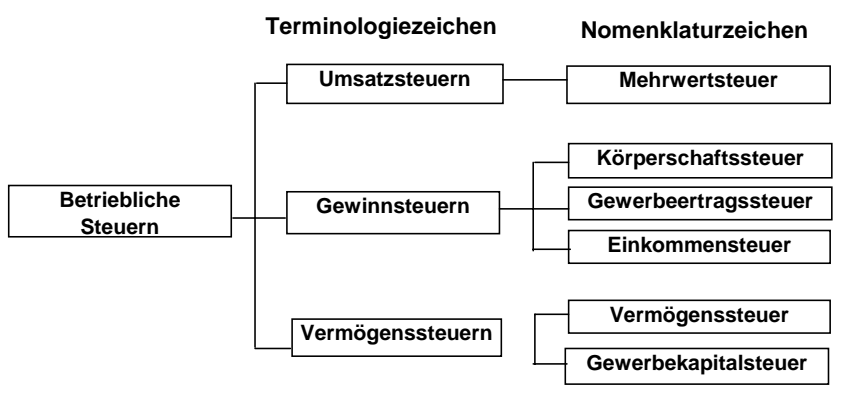

Abb. 1: Terminologisches Subsystem betrieblicher Steuern nach Morgenroth (1993: 58).

Aus diesen Ausführungen lässt sich entnehmen, dass ein hohes Maß an Vorwissen zum fachlichen Sachverhalt vorhanden sein muss, um Erklärungen zur Fachlexik verstehen zu können (vgl. Baumann 1993: 419). Dieses Vorwissen kann an Hand der aufgezeigten Mittel unterstützt werden, indem Textbearbeitungshilfen vorgestellt werden bzw. indem die Angst vor zu langen Fachtexten mit Hinweis auf die bereits vorhandene Kenntnis der einzelnen Wörter und seiner strukturellen Gliederung dem ausländischen Studierenden genommen werden kann.

(3.) Die Kenntnis von Fachtextstrukturen erweist sich ebenfalls als hilfreich bei der Rezeption von Fachtexten. Die Struktur der Fachtexte ist eine spezifische, deren Erfassung wesentlich zur Inhaltsverständniserleichterung beiträgt. Dabei zeichnen sich Fachtexte

[...] wegen der hohen Anforderungen an die Präzision der in (ihnen) enthaltenen Mitteilungen oft durch Besonderheiten in der Makrostruktur, in der Kohärenzbeziehung zwischen seinen Elementen und in seinem Bestand an syntaktischen, lexikalischen, morphologischen und graphisch/phonetischen Einheiten [...] (Hoffmann 1988: 119)

aus, welches jedoch in unterschiedlichem Maße für die einzelnen Fachtextsorten gilt. Daher verfolgt die Fachtextlinguistik relativ einheitlich die Klassifizierung von Fachtextsorten. Ziel ist es, durch methodisches Vorgehen die theoretischen Grundannahmen zu bestätigen, wie zum Beispiel:

- dass Fachtexte eine strukturell-funktionale Einheit bilden;

- dass der Fachlichkeitsgrad durch die spezifische fachsprachliche Kommunikationssituation bestimmt wird;

- dass sich der Fachlichkeitsgrad der Kommunikation aus einer wechselseitigen Beziehungen aus der strukturell-funktionalen Einheit von Fachtexten und der Kommunikationssituation ergibt; und

- dass das funktionale Zusammenwirken von Fachtextinterna und -externa die unterschiedlichen Arten der Fachtexte ermöglicht, die nach ihren wesentlichen Kennzeichen $\mathrm{zu}$ fachübergreifenden Fachtextsorten verallgemeinert werden 
können dass sich der Fachlichkeitsgrad der Kommunikation aus einer wechselseitigen Beziehungen aus Punkt eins ergibt (vgl. Baumann 1992: 75).

Vertreter der Fachtextlinguistik entwickelten verschiedene Wege, Fachtexte zu klassifizieren und zu strukturieren, um sie den Adressaten zugänglicher zu gestalten. Eine wesentliche Bedeutung für die Fachtextstrukturkenntnis ist das Wissen über die Makro- und Mikrostruktur von Fachtexten. So haben sich Analysemodelle mit dem Fokus auf die Makrostruktur linear aufeinander aufbauend entwickelt. Solche Modelle reichen von der textsemantischen Untersuchung nach Agricola (1979), van Dijk (1980) und Viehweger (1976) über die stilistische nach Gläser (1978, 1990) und kumulative nach Hoffmann (1983) zur strukturell-funktional orientierten Betrachtung der Makrostruktur von Fachtexten nach Arntz (1990). Letztere wurde von Baumann (1992, 1996, 2000) erweitert zur Untersuchung der Makrostruktur an Hand von Gliederungssignalen. Das Ziel dieser Modelle ist eine ganzheitliche Betrachtung der Fachtexte, wobei „Fachtexte als sozial und funktional-kommunikativ bestimmte, sachlogisch gegliederte und semantisch strukturierte, linear-sequentiell sowie hierarchisch organisierte sprachliche Einheiten zu erfassen“ (Baumann 1992: 76) sind.

Während die Modelle dazu dienen, die Textgroßstruktur (Makro-) zu vermitteln, müssen die Fachtexte auch auf der Ebene der Mikrostruktur analysiert werden. Die Mirkostruktur unterscheidet sich von der Makrostruktur dahingehend, dass sie nicht die gesamten Sätze zu Teiltexten analysiert, sondern die einzelnen Sätze und deren Bildungssequenzen selbst. Sie stellt eine Feingliederung dar, deren Form und Funktion sich im Allgemeinen aus der Funktion der entsprechenden Teiltexte ergibt (vgl. Roelke 1999: 94). Hierbei spielen vor allem Thema-RhemaStrukturen, Frage-Antwort-Konstruktionen, verschiedenartige Schlussverfahren sowie Rekurenz und Isotopie eine Rolle. Auf der Ebene der Mikrostruktur können aber auch Textverknüpfungsmerkmale analysiert werden wie:

- Zeitfolge,

- Satzeinleitende Konjunktionen oder

- Verwendung von Satzadverbien.

Die aus der Mikrostruktur gewonnenen Informationen „organisieren“ wiederum den inhaltlichen Zusammenhang für die Makrostruktur (vgl. Schramm 2001: 103), was einen rekursiven Vorgang darstellt.

Aus der theoretischen Beschreibung der wesentlichen Merkmale von Fachtexten ist erkennbar, dass von ausländischen Studierenden eine detaillierte Kenntnis der Gestaltung deutscher Fachtexte hinsichtlich nachfolgend zusammengefasster Kriterien erwartet wird:

1. Bewusstmachung der Integration von Gemeinsprache in der Verwendung von Fachsprache

2. Fachterminologische Reduktion durch Terminologien und Nomenklatur 
3. Wahrnehmung der verschiedenen Fachtextsorten und Unterscheidung derer hinsichtlich ihres Aufbaus und der Art und Weise ihrer Inhaltspräsentation

4. Bewusstmachung der Gliederung von Fachtexten durch Erarbeitung von Strukturmerkmalen auf Mikro-/Makroebene.

Dieser Erwartungsdruck führt unweigerlich zu einem Fehlverhalten bei der Rezeption von Fachtexten. Dabei treten entsprechende Leseschwierigkeiten auf, die anschließend erläutert werden sollen.

\section{Wie können die Fachtextrezeptionsprobleme der ausländischen Studierenden im Rahmen der Dissertation nachgewiesen werden?}

Die Nachweismöglichkeit von Leseproblemen ausländischer Studierender bei der Rezeption von wirtschaftswissenschaftlicher Fachtexte erfolgt auf der Grundlage einer von Schramm (2001) erarbeiteten Analysemöglichkeit, sogenanntes inkongruentes Verstehen zu dokumentieren. Schramm (2001) untersuchte in ihrer Studie Psychologiestudenten bei der Rezeption englischsprachiger Fachlektüre in Form mit Hilfe des lauten Denkens. Die aus den Denkprotokollen gewonnenen Aussagen hat Schramm hinsichtlich der Autor-Leser-Kooperation bewertet, um Aussagen zum kongruenten und nichtkongruenten Verstehen ableiten zu können. Die Basis der Analyse von kongruentem und inkongruentem Verstehen bildet dabei die zerdehnte Sprechsituation (nach Ehlich 1984). Kongruentes Verstehen basiert auf der Übernahme der Wissensvermittlungsabsicht des Autors durch den Leser und des damit verbundenen leserseitigen Wissensaufbaus. Inkongruentes Verstehen beschreibt das Gegenteil, wenn die vom Autor antizipierten Wissenslücken der Leser von ihnen nicht erkannt und ,gefüllt“ werden können, sei es aus Gründen einer anderen Textinformationsaufnahmeabsicht oder sei es aus sprachlichen Gründen (funktionale und inhaltliche Inkongruenz). Die von Schramm entwickelte Analysemethode der Daten kann für das Anliegen des vorliegenden Dissertationsprojektes übernommen und gegebenenfalls erweitert werden (makrostrukturelle Inkongruenz). Die auf dieser Grundlage stattfindende Datenauswertung kann das Untersuchungsziel dieser Arbeit (Verstehensprobleme ausländischer Studierender bei der Rezeption von wirtschaftswissenschaftlichen Fachtexten) realisieren und soll an dieser Stelle bezüglich des inkongruenten Verstehens ausführlicher beschreiben werden.

Lutjeharms (1988: 24) markiert eine Grundlage für die Diagnostik von Leseschwierigkeiten in der „Fehleranalyse [...] bei der Rezeption“. Schramm greift diese Systematik auf und benutzt zur Beschreibung der Fehler/Missverständnisse den Begriff des inkongruenten Verstehens, der deutlich eine Abweichung des idealen kongruenten Verstehensverlaufes kennzeichnet, indem die ,identifizierten und integrierten leserseitigen Wissenselemente den verbalisierten autorenseitigen Wis- 
senselementen nicht entsprechen“ (2001: 327). Dabei unterscheidet sie zwei Typen des inkongruenten Verstehens: die inhaltliche und die funktionale Inkongruenz (2001: 329ff).

Die funktionale Inkongruenz bezieht sich auf die vom Autor im Text angelegte Handlungsqualität und die entsprechende leserseitige Rekonstruktion der Handlungsqualität. Die Leser schreiben den autorenseitigen Verbalisierungen eine andere als die angelegte Handlungsqualität zu oder segmentieren die Handlungsschritte an Stellen, die vom Autor nicht vorgesehen worden sind. Als funktionale Inkongruenz können folgende leserseitigen Handlungen angesehen werden (2001: 363ff.):

- inkongruente Leseabsichten (der Leser verfolgt eine anderes Ziel als der Autor: Spezifische Suche nach bestimmten Informationen für Seminararbeiten);

- Finden von unbekannten Wörtern, um diese nachzuschlagen und kontextuell eingebunden zu lernen;

- Kritisches Hinterfragen von Fakten, wenn das Vorwissen und die Erwartungshaltung der Leser nicht mit dem vom Autor vermittelten Wissen harmonieren.

Es handelt sich hierbei um inkongruentes funktionales Verstehen, da die vom Autor antizipierte Leseabsicht leserseitig ignoriert wird.

Hingegen bezieht sich die inhaltliche Inkongruenz auf die propositionalen Gehalte der autorenseitigen Verbalisierung und die leserseitige Wissensstruktur. Man kann dann von einer inhaltlichen Inkongruenz sprechen, wenn ein verbalisiertes Wissenselement X nicht dem entsprechenden Wissenselement X' entspricht. Sie sind auf einfache lexikalische oder grammatikalische „Fehler“ des Lesers zurückzuführen, aber auch auf wissensbedingte Inkongruenzen bezüglich des (nicht)vorhandenen Vorwissens und können wie folgt klassifiziert werden (vgl. Schramm 2001: 331; Lutjeharms 1988: 223, 247f.):

- lexikalisch bedingte Inkongruenz

- lexikalische Interferenzen der Muttersprache

- lexikalische inkongruente Rezeption von Fachtermini, die als solche nicht erkannt werden

- Nicht-Berücksichtigung unbekannter Vokabeln bei der Entnahme von propositionalen Gehalten

- Nicht-Ausnutzung graphischer Information des Wortendes

- fehlendes Vorwissen

- vorhandenes Vorwissen.

Diese inhaltlichen Inkongruenzen beeinflussen die weitere Lesehandlung negativ, da das aufzubauende Wissen konsistent und kohärent an dieses inkongruente Wissen angelagert wird. D. h., ist ein Handlungsschritt bereits von inkongruentem Verstehen gekennzeichnet, kann kein neues Wissen aufgebaut werden, da die Inkongruenz alle weiteren Handlungsschritte überlagert. 
Dieser von Schramm typisierten Inkongruenz des Verstehens kann noch eine weitere hinzugefügt werden, die der ,strukturellen Inkongruenz“, die sich auf die autorenseitig bevorzugte Textstruktur (Makrostruktur) bezieht und leserseitig nicht identifiziert werden kann. Darunter werden die in Abschnitt 2 erarbeiteten Fachtextverstehenskompetenzen hinsichtlich der strukturellen Verarbeitung (Mikro-/Makrostruktur) verstanden. Besonders auf der Ebenen der Makrostruktur können Inkongruenten abgeleitet werden, da inhaltsabhängige Textteile an unterschiedlichen Stellen des Gesamttextes wieder aufgenommen, verknüpft, weitergeführt und ergänzt werden. Wird dies vom Leser verkannt, fehlen ihm wichtige Informationen, um den Lösungsweg nachzuvollziehen und neues Wissen aufzubauen.

Die Untersuchung dieser Fachtextrezeptionsschwierigkeiten erfolgt im Rahmen der Dissertation auf der Basis der folgenden Forschungsfragen:

\section{Welche Leseprobleme können aus der Theorie abgeleitet und empirisch mithilfe einer Kategori- sierung erfasst werden?}

Unter dieser Fragestellung sollen in umfangreicher Form die in der bestehenden Forschung erarbeiteten unterschiedlichen Verstehensprobleme beim Rezipieren von Fachtexten zusammengefasst werden.

\section{Kann kongruentes/inkongruentes Verstehen nachgewiesen werden?}

Es soll damit der Frage nachgegangen werden, ob bei den Fachstudenten der Wirtschaftswissenschaften (Versuchspersonen) überhaupt eine Art der theoretisch recherchierten Textverständnisprobleme vorliegt und die ermittelten Daten Schlüsse vorrangig auf das inkongruente Verstehen zulassen. Die primär damit verbundenen Fragen wären: Kann dieses Modell, das Schramm (2001) für die Verarbeitung der Denkprotokolle aufgestellt hat, auch auf die hier stattgefundene aufgabengeleitete Fachtextrezeption übertragen werden? Ist diese Untersuchungsvariante auch auf die Arbeit mit wirtschaftswissenschaftlichen Texten übertragbar? Lassen die schriftsprachlichen Äußerungen ein kongruentes / inkongruentes Verstehen nachweisen?

\section{Können ermittelte Ergebnisse Kategorisiert und Katalogisiert werden?}

Im Rahmen dieser Fragestellung sollen die erarbeiteten Kategorien explorativ überprüft werden mit dem Ziel, eine Erweiterung oder Gewichtung der Kategorien vorzunehmen. Es sollen die Daten dahingehend ausgewertet werden, in welcher Art und Häufung inkongruentes Verstehen auftritt (Kategorisierung). Des Weiteren soll der Frage nachgegangen werden, ob die Erkenntnisse eine Katalogisierung des inkongruenten Verstehens ermöglichen, als Grundlage für die Interaktion in der professionellen Lehre (Dozenten-Studenten-Fachtext-Interaktion) erprobt werden kann.

Die empirische Untersuchung basiert auf einer quantitativen-qualitativen Analyse der Daten. Quantitativ ist das empirische Vorgehen im Rahmen einer deskriptiven 
Untersuchung, die die Häufigkeiten der auftretenden Leseinkongruenzen herausfiltern soll. Qualitativ wird empirisch geforscht, da die erhaltenen Daten bezüglich des Auftretens des inkongruenten Verstehens untersucht und interpretiert werden müssen.

Die Erhebung der Daten vollzieht sich auf der Basis von aufgabengesteuerten Leseverstehenskontrollen an Originaltexten aus dem Studium der Wirtschaftswissenschaften zum Thema „Globalisierung“. Sie stellten den Inhalt des angebotenen Seminars „Lesekurs Wirtschaftsdeutsch“ dar. Dieses Seminar wurde für ausländische Wirtschaftsstudenten über fünf Semester zu je 30 Doppelstunden (im Folgenden mit DS abgekürzt) angeboten. Inhaltlich orientierte sich die Seminararbeit an den Themen der Fachtextverarbeitung (Fachtextstrukturanalyse, lesestrategische Herangehensweisen), die in 15 DS theoretisch-einleitend besprochen und in weiteren 15 DS selbständig an ausgegebenen Materialien (Aufgaben) geübt und erarbeitet werden sollten. Diese Materialien, die aus einem Einstufungstest, vier entsprechenden Fachtextrezeptionen und einem Abschlusstest bestanden, waren die Ausgangsbasis für die Analyse der Leseschwierigkeiten.

Die Zahl der Versuchspersonen (Probanden) ergibt sich aus dem aufgenommen Datenmaterial, das aus 72 Arbeiten von ausländischen Studierenden der Wirtschaftswissenschaften besteht. Die Probanden sind Studierende in den Fächern Betriebswirtschaftslehre, Wirtschaftsrecht, Wirtschaftsingenieurwesen, Internationale Beziehungen, Kultur und Management sowie Wirtschaftsinformatik. Sie kommen vorrangig aus Polen, aber auch aus Russland, Rumänien, Tschechien, der Slowakei, Finnland, Frankreich, China, Bulgarien, Ungarn und Kasachstan. Ihr Sprachniveau ordnet sich bei sehr guten Deutschkenntnissen auf der Stufe C1 bis C2 des Europäischen Referenzrahmens ein.

Die Dateninterpretation erfolgte qualitativ in Anlehnung an die von Schramm (2001) erarbeitete Datenanalyse zur Herausarbeitung des inkongruenten Verstehens. Diese Datenanalysevariante muss jedoch im Hinblick auf die hier vorliegende Datenreihe ergänzt und auch erweitert werden. Die Analyse der Daten sieht folgendermaßen aus und verdeutlicht den Forschungsansatz, inkongruentes Verstehen bei der Fachtextrezeption ausländischer Studierender der Wirtschaftswissenschaften nachzuweisen. ${ }^{\mathrm{v}}$ 


\begin{tabular}{|c|c|}
\hline \multicolumn{2}{|c|}{ Inhaltliche Inkongruenz } \\
\hline Texta & $\begin{array}{l}\text { „...einer komplexen Realität, welche sich auf Deutschland bezogen darin zeigt, } \\
\text { dass etwa } 40 \text { Mio. Haushalte und etwa } 2 \text { Mio. Unternehmungen existieren. Be- } \\
\text { zeichnet man Unternehmungen mit weniger als } 500 \text { Beschäftigten als kleine und } \\
\text { mittlere Unternehmungen (KMU), so liegt ihr Anteil bei über } 99 \% \text { aller Unter- } \\
\text { nehmungen. Bezeichnet man nur die Unternehmen mit weniger als } 10 \text { Beschäftig- } \\
\text { ten als KMU, so liegt ihr Anteil immerhin noch bei } 75 \% \text {. (Herdzina, Klaus: Ein- } \\
\text { führung in die Mikroökonomie. München: Franz Vahlen, 2002: 19f.) }\end{array}$ \\
\hline Aufg & Stellen Sie die komplexe Realität für Deutschland dar! \\
\hline \multirow{2}{*}{$\begin{array}{l}\text { Vp A } \\
\text { Wirt.-Jura } \\
\text { (PL, 22 J.) } \\
\text { nachweisbare } \\
\text { (In)kongruen- } \\
\text { zen }\end{array}$} & $\begin{array}{l}\text { „Nach dem Lesen kennt man schon viele Arten von Unternehmen und Haushalten, die Interak- } \\
\text { tionen zwischen ihnen und die weiter Überblick, d.h. regionale Zugehörigkeit. Das alles wurde } \\
\text { aber sehr vereinfacht. Um genaue Realität zu sehen, muss man viel mehr beschreiben. Sowohl die } \\
\text { Ursachen des Entstehens sind sehr wichtig, als auch die Koordinationsmechanismen, die erlauben } \\
\text { für gute Exstierung." }\end{array}$ \\
\hline & $\begin{array}{l}\text { - Darstellung der Antwort zeigt, dass Komplex erfasst wurde und sich für die } \\
\text { Beantwortung eigene Gedanken gemacht wurden } \\
\text { - Es liegt hier jedoch eine makrostrukturelle Inkongruenz vor: Zum einen wurde } \\
\text { nicht erkannt, welcher Textteil die entsprechende Antwort auf die Frage liefert } \\
\text { (makrostrukturelle Inkongruenz), } \\
\text { - zum anderen betrachtet die Vp die Aufgabe sehr kritisch und versucht auf } \\
\text { eigene Lösungen zu kommen (aufgabenbezogene Inkongruenz) }\end{array}$ \\
\hline \multirow{2}{*}{$\begin{array}{l}\text { Vp B } \\
\text { BWL } \\
\text { (PL, 22 J) } \\
\text { nachweisbare } \\
\text { (In)kongru- } \\
\text { enzen }\end{array}$} & $\begin{array}{l}\text { „Die vorgestellten Kreislaufmodellen sind vereinfachte Abbilder einer komplexen Realität. Sie } \\
\text { zeigen, wie Wirtschaft funktioniert. Kurz, gesagt: eine gleichgewichte Kreislaufsituation muss } \\
\text { dadurch gekennzeichnet sein, dass in jeden Pol ebenso viele Mittel hineinfließen wie aus ibm } \\
\text { herausfließen. Und die Frage ist, ob Sparen gleich Investieren, Staatseinnahmen gleich Staatsaus- } \\
\text { gaben und Export gleich Import sein müssen." }\end{array}$ \\
\hline & $\begin{array}{l}\text { - Vp beantwortet die Frage mit eigenen Worten } \\
\text { - ABER: sie entspricht nicht der Aufgabenstellung } \\
\text { - Liegt hier eine makrostrukturelle Inkongruen₹ vor: es wurde die Antwort auf die } \\
\text { Frage nicht im Text gefunden (Textgliederungsintoleran`) }\end{array}$ \\
\hline \multirow[b]{2}{*}{$\begin{array}{l}\text { Vp C } \\
\text { BWL } \\
\text { (ČS, } 20 \mathrm{~J} .) \\
\text { nachweisbare } \\
\text { (In)kongru- } \\
\text { enzen }\end{array}$} & „In Deutschland existieren etwa 40 Mio. Haubalten und 2 Mio. Unternehmungen." \\
\hline & $\begin{array}{l}\text { - sehr verknappte Antwort } \\
\text { - Vp ist in der Lage, das Wesentliche eines Textes herauszufiltern } \\
\text { - Aber: funktionale Inkongruenz, da sie dem weiteren Handlungsplan des Autor } \\
\text { nicht folgt (Lösungsweg präsentieren und neues Wissen vermitteln, sondern } \\
\text { diesen bereits am Übergang von Stufe 2, der Verbalisierung des Nicht- } \\
\text { Gewussten, zur Stufe 3, Verbalisierung des Lösungsweges“ abbricht. Es hat } \\
\text { den Anschein, dass die Vp erkannt hat, dass sie eine Wissenslücke hat (Kom- } \\
\text { plexe Realität in Deutschland), sie diese Wissenslücke mit der Vermittlung des } \\
\text { Wissenselementes (Dtl. existieren } 40 \text { Mio. Haushalte und } 2 \text { Mio Unternehmun- } \\
\text { gen) als „gefüllt" betrachtet und hier den weiteren Lösungswegprozess zur } \\
\text { Vermittlung von neuem Wissen (Stufe } 3 \text { und 4) als beendet ansieht. }\end{array}$ \\
\hline
\end{tabular}




\begin{tabular}{|c|c|}
\hline $\begin{array}{l}\text { Vp D } \\
\text { IBZ } \\
(\mathrm{L}, 23 \mathrm{~J} .)\end{array}$ & $\begin{array}{l}\text { „In Deutschland gibt es 40. Mio. Haushalte und } 2 \text { Mio. Unternebmungen. Zwischen den } \\
\text { Unternehmungen unterscheidet man KMU (kleine und mittlere Unternebmungen) und große } \\
\text { Unternehmungen. Laut der verschiedenen Kriterien gibt es in Deutschland } 99 \% \text { KMU (bis } \\
\text { zu } 500 \text { Arbeiter) und 1\% Großunternebmungen oder } 75 \% \text { KMU (bis ₹u } 10 \text { Arbeiter) und } \\
25 \% \text { Großunternehmungen." }\end{array}$ \\
\hline $\begin{array}{l}\text { nachweisbare } \\
\text { (In)konkru- } \\
\text { enzen }\end{array}$ & $\begin{array}{l}\text { - Vp ist bis zur letzten Stufe den Ausführungen des Autors gefolgt (kongruentes } \\
\text { - Ierstehen) } \\
\text { - In der Handlungsstufe „Verbalisierung neuen Wissens“ ist jedoch ein } \\
\text { funktionales inkongruentes Verstehen aufgetreten } \\
\text { - Die Vp erkennt nicht, dass die Darstellung der KMU mit weniger als } 10 \\
\text { Beschäftigten eine Weiterführung der Darstellung der gesamten KMU - } \\
\text { - Darstellung von } 99 \% \text { mit bis zu } 500 \text { Beschäftigten ist. Kurz: Von den } 99 \% \\
\text { KMU bis } 500 \text { Beschäftigten sind } 75 \% \text { davon KMU bis } 10 \text { Beschäftigte } \\
\text { - Für die Vp ist die Darstellung der } 99 \% \text { KMU ein abgeschlossener Handlungs- } \\
\text { schritt, dem ein neuer (75\% KMU mit max. } 10 \text { Beschäftigten) folgt }\end{array}$ \\
\hline
\end{tabular}

\section{Welche Relevanz haben die vorläufigen Untersuchungsergebnisse?}

Die bisher im Rahmen der Vorarbeit festgehaltenen Evaluationsergebnisse in den genannten Kursen deuten darauf hin, dass sich durch die gezielte Förderung des Rezipierens von Fachtexten unter spezifischer Aufgabenstellung zur Erlangung einer Fachtextrezeptionskompetenz tatsächlich gewisse Wiederholungsmuster des inkongruenten Verstehens nachweisen lassen, die zum einen kulturellen Hintergrundes sind, zum anderen aber auch in sprachlichen Defiziten zu verorten sind (inhaltliche Inkongruenz), vorrangig sind jedoch funktionale und makrostrukturelle Inkongruenzen nachweisbar.

Betrachtet man diese Ergebnisse im Rahmen einer Langzeitstudie, so sind im Verlauf der fortschreitenden Fachtextrezeption Fertigkeitsentwicklungen nachweisbar, die anfänglich häufig auftretende Inkongruenzen deutlich verringert nachweisbar werden lassen. Längerfristig sind diese Daten intensiver auszuwerten und zu prüfen. Es zeichnet sich bereits ab, dass die Ergebnisse in zweierlei Hinsicht betrachtet werden müssen: Zum einen sind die Fachtextrezeptionen als solche einzeln in ihrer Erfüllung zu betrachten, da hier die inhaltlichen, funktionalen (makro)strukturellen Inkongruenzen nachgewiesen werden können. Andererseits, dem Longitudinalcharakter der Studie folgend, verringert sich das Auftreten von Inkongruenzen und ein kongruentes Verstehen wird immer häufiger nachweisbar.

Davon ausgehend, dass Fachtextrezeptionsschwierigkeiten einen erfolgreichen Studienabschluss verhindern können und damit sowohl den deutschen Studienalltag als auch entsprechende berufliche Chancen im Heimatland beinträchtigen werden, kann durch die Weiterführung der Untersuchung ein Beitrag zur Integrationserleichterung ausländischer Studierender in deutsche Fachstudiengänge geleistet werden. Aus didaktischer Perspektive wäre die Entwicklung von Handlungskatalo- 
gen für Lehrende, wie mit entsprechenden Leseproblemen auf Fachtextebene verfahren werden könnte, um selbständige Studierkompetenzen der Lerner zu entwickeln, empfehlenswert. Einerseits erleichtert es den Fachdozenten den Umgang mit ihren ausländischen Studierenden, indem unterschiedliche, landestypische Verfahrensweisen kennen gelernt werden können, die auch auf Seiten der Dozenten Verständnis erwirken und die Erwartungshaltung am Anfang nicht als strikt, sondern sich über die Zeit entwickelnd charakterisiert. Andererseits können durch katalogisierte Fachtextrezeptionsschwierigkeiten auch Hilfestellungen bei der Textauswahl und -aufbereitung aufgelistet werden, die ein Zusammenspiel von Dozent und Student positiv unterstützen.

\section{Literatur}

Baumann, Klaus-Dieter (1992): Integrative Fachtextlinguistik. Tübingen: Narr.

Baumann, Klaus-Dieter (1993): Ein komplexes Herangehen an das Phänomen der Facblichkeit von Texten. In: Bungarten, Theo (Hg.): Fachsprachentheorie. Band 1: Fachsprachliche Terminologie, Begriffs- und Sachsysteme, Methodologie. Tostedt: Attikon Verlag, 395429.

Baumann, Klaus-Dieter (1995): Fachsprachen und Fachsprachendidaktik. In: Bausch, KarlRichard; Christ, Herbert \& Krumm, Hans-Jürgen (Hrsg): Handbuch Fremdsprachenunterricht. Tübingen/Basel: Francke, 332-338.

Baumann, Klaus-Dieter (1996): Fachtextsorten und Kognition - Erweiterungsangebote an die Fachsprachenforschung. In: Kalverkämper, Hartwig \& Baumann, Klaus-Dieter (Hrsg.): Fachliche Textsorten. Komponenten - Relationen - Strategien. Tübingen: Narr, 355-388.

Baumann, Klaus-Dieter (2000): Die Entwicklung eines integrativen

Fachsprachenunterrichts - eine aktuelle Herausforderung der Angewandten Linguistik. In: Baumann, Klaus-Dieter \& Kalverkämper, Hartwig (Hrsg.): Sprachen im Beruf: StandProbleme - Perspektiven. Tübingen: Narr.

Blei, Dagmar (1993): Fachlichkeit und Fachsprachlichkeit in didaktischer Sicht. In: Schröder, H. (Hrsg.): Fachtextpragmatik. Tübingen: Narr, 395-406.

Bolten, Jürgen: Fremdsprache Wirtschaftsdeutsch: Bestandsaufnahme und Perspektiven. In: Müller, Bernd-Dietrich (Hrsg.): Interkulturelle Wirtschaftskommunikation. München: Iudicum, 71-91.

Brinker, Klaus (2005): Linguistische Textanalyse. Eine Einführung in die Grundbegriffe und Methoden. Berlin: Schmidt.

Buhlmann, Rosemarie \& Fearns, Anneliese ( $\left.{ }^{6} 2000\right)$ : Handbuch des Fachsprachenunterrichts. Unter besonderer Berücksichtigung naturwissenschaftlich-technischer Fachsprachen. Tübingen: Narr.

Christmann, Ursula \& Groeben, Norbert (1996): Die Rezeption schriftlicher Texte. In: Günther, Hartmut \& Ludwig, Otto (Hrsg.): Schrift und Schriftlichkeit - Writing and its use. 
Ein interdisziplinäres Handbuch internationaler Forschung - An interdisciplinary handbook of international research. Berlin/New York: De Gruyter, 1536-1545.

Ehlich, Konrad (1984): Zum Textbegriff. In: Rothkegel, Annelie \& Sandig, Barbara (Hrsg.): Text - Textsorten - Semantik. Hamburg: Buske, 9-25.

Gläser, Rosemarie (1978): Methoden zur Erforschung von Stilmerkmalen in den Fachsprachen des Englischen. In: Zeitschrift für Phonetik, Sprachwissenschaft und Kommunikationsforschung 2, 159-169.

Gläser, Rosemarie (1990): Fachtextsorten im Englischen. Tübingen: Narr.

Gläser, Rosemarie (1993): Textsortenvergleich im universitären Fremdsprachenunterricht. In: Morgenroth, Klaus (Hrsg.): Methoden der Fachsprachendidaktik und-analyse: deutsche Wirtschafts- und Wissenschaftssprache. Frankfurt/M. [u.a.]: Lang, 17-38.

Grzesik, Jürgen (1990): Textverstehen lernen und lehren. Geistige Operationen im Prozess des Textverstehens und typische Methoden für die Schulung zum kompetenten Leser. Stuttgart: Klett.

Heinemann, Margot (2000): Textsorten des Bereiches Hochschule und Wissenschaft. In: Brinker, Klaus et al. (Hrsg.): Text-und Gesprächslinguistik. Ein internationales Handbuch zeitgenössischer Forschung. 1. Halbband, Berlin, 702-709.

Heuberger, Katharina (1997): Wirtschaftsdeutsch und seine Vermittlung: eine Bestandsaufnahme. Tostedt: Attikon Verlag.

Hoffmann, Lothar (1988): Vom Fachwort zum Fachtext: Beiträge zur angewandten Linguistik. Tübingen: Narr.

Höhne, Steffen (1991): Morpho-graphematische Probleme in der betrieblichen Kommunikation. In: Müller, Bernd-Dietrich (Hrsg.): Interkulturelle Wirtschaftskommunikation. München: Iudicium, 433-450.

Ihle-Schmidt, Lieselotte (1983): Studien zur französischen Wirtschaftssprache. Frankfurt/Main [u.a.]: Lang.

Lutjeharms, Madeline (1988): Lesen in der Fremdsprache. Versuch einer psycholinguistischen Deutung am Beispiel Deutsch als Fremdsprache. Bochum: AKS.

Morgenroth, Klaus (1993): Wortschatzanalyse als Orientierungshilfe im Fachsprachenunterricht. In: Ders. (Hrsg.): Methoden der Fachsprachendidaktik und -analyse: deutsche Wirtschafts- und Wissenschaftssprache. Frankfurt/Main [u.a.]: Lang, 47-65.

Ohnacker, Klaus (1992): Die Syntax der Fachsprache Wirtschaft im Unterricht Deutsch als Fremdsprache. Frankfurt/Main [u.a.]: Lang.

Roelcke, Thorsten (1999): Fachsprachen. Berlin: Schmidt.

Satzger, Axel (1993): Sprachliches Handeln im Fachtext. Ein Analysekonzept. In: Bungarten, Theo (Hrsg.): Fachsprachentheorie. Band 1: Fachsprachliche Terminologie, Begriffsund Sachsysteme, Methodologie. Tostedt: Attikon Verlag, 299-333.

Schnotz, Wolfgang (1996): Aufbau von Wissensstrukturen. Untersuchungen zur Kobären₹bildung bei Wissenserwerb mit Texten. Weinheim: Beltz. 
Schramm, Karen (2001): L2-Leser in Aktion. Der fremdsprachliche Leseprozess als mentales Handeln. Münster [u.a.]: Waxmann.

Steinmetz, Maria (2000): Fachkommunikation und DaF-Unterricht. Vernetzung von Fachwissen und Sprachausbildung am Beispiel eines Modellstudienganges in China. München: Iudicium.

Woll, Artur (1993): Wirtschaftslexikon. München/Wien: Oldenbourg.

i An der Universität Leipzig waren zum Wintersemester 2007/2008 ca. 30.000 Studierende eingeschrieben, wobei 4.800 Neuimmatrikulierte registriert werden konnten. Die Rate der ausländischen Immatrikulierten lag dabei mit 1.144 Studierenden bei rund 20\%. [www.db.uni-leipzig. de/aktuell/index.html; 22.09.2008]

ii Schramm (2001: 327-397) widmet sich der „Fehlersuche“ zur Entstehung von Missverständnissen bei der Rezeption von Fachtexten in Form eines ganzen Kapitels. Sie stellt jedoch voran, dass der Begriff des Missverständnisses und damit auch des Fehlers an dieser Stelle präzisiert werden muss und kreiert den Begriff des ,inkongruenten Verstehens“ (S. 327). Dieser Begriff ist auch auf diese Arbeit zu übertragen, da er den Autor-Leser-Verstehensprozess kennzeichnet. Der Autor antizipiert Wissenslücken des Lesers, bei deren Schließung aber leserseitiges Nicht-Verstehen auftreten kann, dass zu einem inkongruenten Verstehen auf der Fachkommunikationsebene zwischen Autor und Leser über den Text erfolgt.

iii Heuberger (1997: 50); Ehlich (2001: 198) beschreibt in seinem Artikel „Deutsche Wissenschaftskommunikation“ eine Entwicklung der Sprache ,[...] die die heutige Wissenschaft im Wesentlichen unterhalten, betreiben und nutzen [soll und damit] unabdingbar für den Erhalt dieser Gesellschaften“ ist. Dies führt im übertragenen Sinne auch zur Situierung der Fachsprache Wirtschaft.

iv Satzger (2001: 169) sieht hier den Fehler in der Fachtextlinguistik, die sich dem schnell wachsenden Bedarf einer Wirtschaftsfachsprache zu langsam nähert und daher eine entsprechende linguistische Fundierung vermissen lässt.

$\mathrm{v}$ Eine Kategorisierung ist zum gegebenen Zeitpunkt der Datenaufnahme und -analyse noch nicht möglich. Hypothetische Annahmen nach der Sichtung des Materials lassen eine Häufung von inkongruentem Verstehen bezüglich makrostruktureller, funktionaler Inkongruenz erkennen. Es muss jedoch diese Annahme erst empirisch gestützt werden, um verlässliche Aussagen treffen zu können. 


\title{
„Später dann, wo ich Stärke und Sprache [hatte], dann hab ich nachg'fragt..." -
}

\author{
Eine kritische Erhebung sprachlicher Bedürfnisse von nostrifizierten \\ Gesundheits- und Krankenschwestern/-pflegern in Österreich
}

\author{
Barbara Haider (Wien)
}

\begin{abstract}
Der vorliegende Artikel geht der Frage nach, welche sprachlichen Bedürfnisse Gesundheits- und Krankenschwestern/-pfleger mit Migrationshintergrund bei ihrem Berufseinstieg in Österreich entwickeln und wie auf diese Bedürfnisse reagiert werden könnte. Für die Untersuchung wurde der Ansatz der kritischen Sprachbedarfserhebung (critical needs analysis) gewählt. Dieser bietet die Möglichkeit, sowohl die subjektiven Bedürfnisse der Krankenschwestern/-pfleger als auch die objektiven Anforderungen im Beruf (Sprachbedarfe) selbst sowie die institutionellen Rahmenbedingungen kritisch zu analysieren und nach Fördermöglichkeiten für die Betroffenen, aber auch nach Veränderungsmöglichkeiten im System zu suchen.
\end{abstract}

\section{Einleitung}

Berufe im Bereich der Gesundheits- und Krankenpflege sind Sprachberufe par excellence, da sie neben einer umfassenden Fachkompetenz auch ein hohes Maß an kommunikativer Kompetenz im Umgang mit PatientInnen, KlientInnen, aber auch KollegInnen im intra- und interdisziplinären Diskurs fordern. Trotz dieses zentralen Stellenwerts der Kommunikation in der Pflege gibt es in Österreich bis dato kein (institutionalisiertes) Sprachförderungsprogramm für Pflegekräfte nichtdeutscher Muttersprache, die in Österreich traditionell einen hohen Anteil der Beschäftigten darstellen. Die derzeit gängige Praxis sieht vor, dass sich Pflegekräfte direkt in der Berufspraxis selbst - durch learning by doing - die für den Beruf notwendigen Sprachkenntnisse aneignen, ohne dabei auf ein entsprechendes Kursangebot zurückgreifen zu können. Diese Vorgehensweise stößt jedoch vor dem Hintergrund der fortschreitenden Qualitätsentwicklung und steigenden Anforderungen - vor allem im schriftlichen Bereich durch die Einführung einer umfassenden Dokumentation des Pflegeprozesses - an Grenzen: Sie stellt eine harte Form des immersiven bzw. sogar submersiven Lernens dar, die auf die Formel „Sink or swim“ (Fthenakis et al. 1985: 316) gebracht werden kann.

Berufsspezifische Deutschkurse für diese Zielgruppe werden, wenn überhaupt, nur punktuell vor allem an Volkshochschulen (VHS) angeboten, wo ich als Kurs- 
leiterin selbst mit dieser Thematik konfrontiert war und aufgrund unzureichender Unterrichtskonzepte und -materialien die dringende Notwendigkeit einer eingehenderen Auseinandersetzung mit diesem Thema sah. Im Folgenden möchte ich einen Ausschnitt der Sprachbedarfs- und -bedürfniserhebung im Berufsfeld der Gesundheits- und Krankenpflege, die ich im Rahmen meiner Dissertation ${ }^{i}$ durchgeführt habe, vorstellen.

Ich werde mich dabei auf einen Teil der erhobenen Sprachbedürfnisse von diplomierten Gesundheits- und Krankenschwestern/pflegern konzentrieren, die bereits mit einer abgeschlossenen Ausbildung nach Österreich gekommen sind und hier ihr Diplom nostrifizieren, d.h. offiziell anerkennen ließen. Anhand der Schilderungen ihres Berufseinstiegs sollen sprachliche Probleme und Herausforderungen in der beruflichen Praxis aufgezeigt werden, die Anhaltspunkte für die Entwicklung von berufsspezifischen Deutschkursen bieten. Gleichzeitig wird der Blick auf Mängel im System von Pflegeeinrichtungen gerichtet, die den (beruflichen) Sprachlernprozess erschweren und im direkten Widerspruch zum fortschreitenden Qualitätsentwicklungsprozess in der Pflege stehen. Diese weite Zugangsweise zu Sprachbedarf, die das gesamte sprachliche und soziale Handlungsfeld berücksichtigt, stammt aus der angloamerikanischen Forschungstradition zu English for Specific Purposes (ESP). Diese hat sich am längsten und bisher ausführlichsten mit der Erhebung und Analyse von Sprachbedarf (needs analysis) ,für spezielle Zwecke“ (z.B. für die Wirtschaft, Medizin, Technik) auseinandergesetzt. Im deutschsprachigen Raum entspricht dieser Disziplin die Fachsprachendidaktik sowie die erst junge Richtung der Berufssprachendidaktik, aus deren Kontext meine Differenzierung zwischen objektiven Sprachbedarfen und subjektiven Sprachbedürfnissen stammt. Ein kurzer Aufriss dieser theoretischen Grundlagen soll im Folgenden den Rahmen meiner Arbeit erläutern.

\section{Von einer deskriptiven zu einer kritischen Sprachbedarfserhebung}

Mit einer Sprachbedarfserhebung (needs analysis) wird - ganz vereinfacht formuliert - versucht herauszufinden, was Lernende brauchen, warum sie eine Sprache lernen, worauf sie vorbereitet werden sollen, durch welche sprachlichen Anforderungen bestimmte Situationen charakterisiert sind, welche Bedürfnisse und Erwartungen an einen Sprachkurs bestehen etc.: „Needs analysis is the process of establishing the what and how of a course" (Dudley-Evans \& St John 1998: 121). Auf Basis der gewonnen Informationen können einerseits allgemeine Empfehlungen für Kursmaßnahmen gegeben oder Richtlinien für Curricula entworfen werden und andererseits kann konkreter Unterricht geplant und durchgeführt werden. Im Zuge dieses Prozesses tauchen allerdings einige Fragen auf, die Richterich folgendermaßen zusammenfasst (Richterich 1983: 1): 
- who decides to identify needs?

- who compiles the information?

- what information?

- on whom?

- how?

- where and when?

- who makes use of the information?

- how?

- to do what?

- in what form? [...]

- how to assess the whole identification procedure?

Beim Versuch, diese Fragen zu beantworten, zeigt sich, dass die Antworten je nach (pädagogischem, methodisch-didaktischem, linguistischem, politischem etc.) Kontext und Ziel der Untersuchung sehr unterschiedlich ausfallen werden. Zunächst sind für die Erhebung von sprachlichen Anforderungen in einem bestimmten Kontext die dem geplanten Unterricht zugrunde liegenden methodischdidaktischen Prinzipien bestimmend. Sie lenken bereits im Vorfeld die Fragestellung, mit der man sich einer zu beschreibenden Sprachsituation nähert, und prägen die jeweils spezifische Herangehensweise (z.B. Sammlung von Themen oder linguistische Untersuchungen) an den Sprachbedarf: Im Kontext von berufsbezogenem Deutschunterricht werden in der aktuellen Diskussion drei methodisch-didaktische Ansätze genannt, die alle auf situations- und kontextgebundenes Lernen abzielen (vgl. Grünhage-Monetti et al. 2003):

a) Der aufgabenorientierte Ansatz (task-based approach) versucht, die Komplexität von realen Kommunikationsprozessen ins Klassenzimmer zu bringen, innerhalb derer die Lernenden aufgefordert sind, bestimmte Aufgaben sprachlich zu „lösen“" und mit Sprache zu handeln (vgl. z.B. Nunan 2004).

b) In einem themenorientierten Ansatz (theme-based approach) sind die Lernenden dazu angehalten, selbst die zu behandelnden Themen - gemeinsam mit der Lehrperson, die Vorschläge und Anhaltspunkte bereit hält und die Auswahl strukturiert - zu bestimmen und bildlich gesprochen in die Klasse zu tragen, z.B. indem sie ihren eigenen Arbeitsablauf darstellen, schriftliche Unterlagen vom Arbeitsplatz mitbringen, etc.

c) Der Szenario-Ansatz (scenario-based approach) basiert schließlich auf diskursanalytischen Auswertungen authentischer Kommunikation in einem bestimmten Kontext. Diese werden zu so genannten Szenarien gebündelt und können als Unterrichtsgrundlage dienen. Unter Szenarien ist eine „erwartbare Abfolge kommunikativer Handlungen" zu verstehen, „die teils als rein sprachliche Handlungen, teils als rein nicht-sprachliche Handlungen und teils in gemischter Form laufen. Szenarien erhalten ihre Kohärenz durch den gewußten sozialen Sinn"“ (Von der Handt \& Stricker 1995: 51; Herv. i.O.). 
Neben den methodisch-didaktischen Prinzipien hat auch das jeweilige Konzept von Sprache Einfluss auf die Identifizierung und Festlegung von Sprachbedarf: Wie weit wird Sprache gefasst? Wird sie nur in ihrer operationalen/funktionalen Dimension gesehen, die rein dem Austausch von Informationen dient, oder wird Sprache weiter gefasst, wie etwa im Konzept von Sprache als sozialer Praxis. Dieses aus Australien stammende Modell (vgl. Durrant \& Green 2000; Green 1988) geht von drei Dimensionen der sprachlichen Praxis aus: der bereits genannten operationalen/funktionalen Dimension und darüber hinaus der kulturellen und der kritischen Dimension. Übertragen auf den Sprachlernbereich können diese wie folgt definiert werden:

The operational/functional dimension enables language users (in our case migrant/ ethnic workers) to exchange information. For example, explaining a default in a machine/product, following instructions, etc. This is the level of formal correctness, as regards vocabulary, grammar, etc.

The cultural dimension accounts for culturally appropriate interaction. For example, using the appropriate register, body language, proxemics, intonation. This is the level of broad cultural appropriateness at the level of "genres": for example, taking or keeping the floor during a group meeting.

The critical dimension involves understanding one's relationship to others in a specific community of practice. It implies recognising and judging the social relevance of the communicative events/acts in a specific community of practice and gives the instrument to make informed choices. The knowledge and the skills connected to this dimension enable the speakers to take on an active role in communication in a specific context: for example, how to take or keep the floor as a middle-aged, Muslim woman in a "white", male-dominated group meeting of the department XY in company Z (Grünhage-Monetti et al. 2003: 13; Herv. d. Verf.).

Diese drei Dimensionen von Sprache sind untrennbar miteinander verknüpft und voneinander abhängig und können daher auch nicht unabhängig voneinander unterrichtet und gelernt werden. Das bedeutet, dass auch in einer Untersuchung der jeweiligen für Lernende zu bewältigenden sprachlichen Zielsituation alle drei Dimensionen berücksichtigt werden müssten und eine Konzentration auf die funktionale, „objektive“ Sprachebene allein nicht ausreicht - vorausgesetzt, die Lernenden sollen überhaupt umfassend auf ein berufliches Handlungsfeld vorbereitet und nicht auf ihre Funktion als reine Arbeitskraft reduziert werden. Hier wird also auch immer die Auftragssituation einer Sprachbedarfserhebung eine zentrale Rolle spielen: Wer finanziert für wen und zu welchem Zweck eine derartige Erhebung und was soll mit einem (berufsspezifischen) Sprachkurs erreicht werden?

Als ein zentraler Schritt in der Diskussion um Sprachbedarfserhebung ist in diesem Zusammenhang die Feststellung anzusehen, dass diese niemals „objektiv“ oder neutral sein kann, sondern durch den jeweiligen (linguistischen, pädagogischen, institutionellen, kulturellen, politischen, etc.) Kontext, in dem sie stattfindet, stets ideologisch gefärbt ist. 
Needs analysis [...] has falsely purported to be neutral and to be simply providing objective taxonomies of necessary skills. In fact, these taxonomies of needs have a hidden ideological basis (i.e., the representation of institutional wants). The traditional brief is now being questioned and calls are made for LSPii not simply to uncritically accept and maintain communicative norms and thus the status quo of linguistically privileged in-groups, but to challenge those norms (Basturkmen 2002: 28).

„Objektive“, rein beschreibende Erhebungen, die als Basis von Sprachunterricht dienen, laufen somit Gefahr, die Zielsituation als unveränderbar anzusehen, an die die Lernenden optimal angepasst werden sollen. Dadurch kommt es jedoch zu einer Reproduktion des dominanten Diskurses, der in dieser Herangehensweise nicht näher hinterfragt wird: „In a descriptive needs analysis [...] the researcher does not look for ways to modify current conditions but instead aims to fit students into the status quo by teaching them to make their behavior and language appropriate“ (Benesch 1996: 727). Im Gegensatz zu dieser Beschreibung des IstZustandes werden in einem kritischen Zugang (critical needs analysis) bestehende Strukturen hinterfragt und es wird nach Reformmöglichkeiten gesucht. „[C]ritical needs analysis acknowledges existing forms, including power relations, while searching for possible areas of change" (ebd.: 732). Die hierarchische Natur von Institutionen und die dadurch entstehende Ungleichheit innerhalb und auch auBerhalb dieses Feldes werden in einer kritischen Sprachbedarfserhebung zu zentralen Themen. Um diesen Kontext in den Blick zu bekommen, ist es unumgänglich, eine weite Herangehensweise, die sich nicht ausschließlich auf z.B. sprachliche Aspekte in einer bestimmten Zielsituation beschränkt, zu wählen und möglichst viele verschiedene Informationsquellen zu nutzen. Auf diese Weise erhält man ein umfassendes Bild, das durchaus aus divergierenden Sichtweisen bestehen kann: "The use of multiple sources [...] shows that different actors in a social setting have different perceptions of similar tasks and situations, which leads them to different objective and felt needs" (Jasso-Aguilar 2001: 44). Für die von mir durchgeführte Erhebung im Berufsfeld der Gesundheits- und Krankenpflege wurden dafür (politische) EntscheidungsträgerInnen, PflegewissenschaftlerInnen, PflegeexpertInnen, pflegerisches Führungspersonal, Unterrichtende der Gesundheits- und Krankenpflegeausbildung bzw. des Nostrifikationslehrgangs iii sowie Pflegepersonal mit migrantischem Hintergrund befragt sowie teilnehmende Beobachtungen im Krankenhaus und Hospitationen im Lehrgang durchgeführt. Besonderes Gewicht wurde dabei den Betroffenen, d.h. den (zukünftigen oder ehemaligen) Lernenden selbst beigemessen; sie sollten zur Sprache kommen und ihre Anliegen und Bedürfnisse formulieren, die möglicherweise im Widerspruch zu offiziellen Anforderungen einer Institution stehen.

Bevor jene Sprachbedürfnisse für das Berufsfeld der Gesundheits- und Krankenpflege vorgestellt werden, sollen die hier bereits verwendeten Begriffe „Bedarf“ und „Bedürfnisse“, die als Übersetzung für den Terminus „,needs“ dienen, noch einmal genauer unter die Lupe genommen und definiert werden. 


\section{Definition von Sprachbedarf(en) und Sprachbedürfnissen}

Im deutschsprachigen Raum finden sich zwei mittlerweile gängige Begriffe, die in das Wortfeld needs fallen: Sprachbedarf(e) und Sprachbedürfnis(se). Erstere werden dabei zumeist mit dem Adjektiv „objektiv“ versehen, während Sprachbedürfnisse ,subjektiv“ sind:iv

Der objektive Bedarf ist gleichzusetzen mit den kommunikativen Anforderungen im berufs- bzw. arbeitsplatzspezifischen Kontext. Im Unterricht wird man wohl immer auch gleichzeitig konfrontiert mit den subjektiven Erwartungen und Bedürfnissen, mit den Motivationen der TeilnehmerInnen - bezogen auf die unterschiedlichen Prozesse. [...] Insofern sind also die subjektiven Kenntnisse, Erfahrungen und Erwartungen sowie die Motivation der TeilnehmerInnen ein wichtiges Korrektiv, um sich mit den objektiven Themen- und Fragestellungen, wie der berufsorientierte Deutschunterricht konkret ausgestaltet werden soll, auseinander zu setzen (Szablewski-Çavuş 2000: 18f.).

In ähnlicher Weise definiert Klepp vom Deutschen Institut für Erwachsenenbildung die beiden Begriffe, wobei er den „objektiven Bedarf“ stärker personalisiert, d.h. als Erwartungen von Leitungsebene an die Sprachkenntnisse der MitarbeiterInnen ausweist:

Wir verwenden diese beiden Ausdrücke [Sprachbedarfe und -bedürfnisse; d.Verf.], um zwei verschiedene Sichtweisen auf die Frage, welche „Sorte“ von Sprache und welche sprachlichen Anforderungen am Arbeitsplatz auftreten, unterscheiden zu können: Sprachbedarfe sollen dabei die Anforderungen und Wünsche aus Sicht der Unternehmer, d.h. sowohl von Vorgesetzten als auch von KollegInnen, Betriebsräten usw. bezeichnen; Sprachbedürfnisse die Wünsche und subjektiven Erfahrungen der LernerInnen selber (Klepp zit. in GrünhageMonetti 2000: 13).

Anhand dieser beiden Erklärungen des „objektiven Sprachbedarfs“ sei auf eine nicht unerhebliche Differenz zwischen tatsächlichen sprachlichen Anforderungen im Job und Erwartungen von Unternehmensseite hingewiesen, die deutlich macht, dass eine umfassende Definition unbedingt beide Aspekte berücksichtigen muss. Während kommunikative Anforderungen an einem bestimmten Arbeitsplatz von außen „objektiv“ betrachtet sehr gering sein mögen, kann es von Arbeitgeberseite her durchaus hohe sprachliche Anforderungen an (potentielle) ArbeitnehmerInnen geben, die nicht unbedingt an die konkreten beruflichen Aufgaben geknüpft sind, sondern z.B. als Selektionskriterium dienen. Eine Beschreibung von Sprachbedarfen muss demnach sowohl die arbeitsplatzbezogene Kommunikation als auch - als Erweiterung bzw. Korrektiv - die Wünsche „aus Sicht der UnternehmerInnen“ integrieren.

Für die von mir durchgeführte Erhebung wurde darüber hinaus noch eine dritte Ebene des Sprachbedarfs eruiert und analysiert, nämlich die theoretischen Anforderungen eines Berufs. Darunter verstehe ich die Inhalte, die in der Ausbildung vermittelt werden, bzw. das Berufsbild oder auch das Leitbild eines Betriebs. Im 
Bereich der Gesundheits- und Krankenpflege werden auf dieser Ebene sehr hohe kommunikative Erwartungen formuliert und wird kommunikative Kompetenz als eine Schlüsselqualifikation angesehen, die jedoch in der Arbeitsrealität oftmals keine Anwendungsmöglichkeit findet.

Die Darstellung von Sprachbedarfen, die alle drei Ebenen berücksichtigt, kann demnach ein in sich widersprüchliches Bild ergeben: zwischen konkreten Anforderungen der Arbeitsrealität und Erwartungen der Unternehmensebene sowie zwischen einem theoretischen Berufsbild und der beruflichen Praxis. Darüber hinaus können sich auch die objektiven Sprachbedarfe und die subjektiven Sprachbedürfnisse der Lernenden voneinander unterscheiden. Letztere sind von ihren persönlichen (Sprach-)Lernerfahrungen, ihren beruflichen Erfahrungen, ihren persönlichen Einstellungen zu und Vorstellungen von einem bestimmten Beruf und nicht zuletzt von ihrer Lernmotivation geprägt. Je konkreter die Berufserfahrungen der Betroffenen sind, desto genauer werden in diesem Bereich ihre Vorstellungen und Wünsche sein, etwa bei einem Deutschkurs direkt am Arbeitsplatz.

Ein erster notwendiger Schritt muss es sein, diese verschiedenen Vorstellungen, Forderungen, Haltungen etc. offenzulegen und klar zu benennen, um dann in einem nächsten Schritt ein Sprachförderungsmodell aushandeln zu können, das den Lernenden einen emanzipierten Umgang mit der jeweiligen beruflichen Situation ermöglicht.

\section{Datengrundlage und methodische Vorgangsweise}

Die Bestimmung der Sprachbedürfnisse im Berufsfeld der Gesundheits- und Krankenpflege beruht auf der Auswertung von qualitativen Interviews mit ehemaligen NostrifikantInnen, d.h. mit Personen, die ihr im jeweiligen Herkunftslandv erworbenes Gesundheits- und Krankenpflegediplom erfolgreich nostrifiziert haben und bereits im Berufsleben stehen. Im Zeitraum von Juni 2003 bis Mai 2004 wurden insgesamt dreizehn halb-standardisierte Interviews mit diplomierten Gesundheitsund Krankenschwestern bzw. -pflegern aus verschiedenen Ländern geführt (s. Übersichtstabelle). Die Interviews wurden anschließend transkribiert, wobei ich mich für eine mittlere Exaktheitsstufe entschied, da in erster Linie die angesprochenen Themen im Vordergrund standen und keine feinstruktur- oder diskursanalytische Fragestellung: Der Text wurde genau wiedergegeben und nicht bereinigt; Aussprachebesonderheiten (Akzente, dialektale Färbungen etc.) wurden nicht festgehalten. Pausen wurden nur bei markant längerer Dauer eingezeichnet [Pause], nonverbale Aspekte oder Störungen des Gesprächs wurden ebenfalls in eckigen Klammern festgehalten.

Für die Auswertung und Interpretation wurde im Hinblick auf das Ziel der Sprachbedarfserhebung, einen ersten Gesamtüberblick über das sprachliche Handlungsfeld der Pflege zu geben, eine themenorientierte Darstellungsweise gewählt, die Schlüsselprobleme der MigrantInnen präsentiert, die ihre Situation treffend 
charakterisieren und die auch in einem berufsspezifischen Deutschkurs berücksichtigt werden müssten (s.o.: themenorientierter Ansatz). Daran müssten sich in weiterer Folge die Erhebung und Aufbereitung von Aufgaben und Szenarien basierend auf linguistischen Untersuchungen (z.B. Genre-Analyse) beruflich relevanter Sprechhandlungen anschließen, um konkrete Kurse durchführen zu können.

Im Zentrum der Interviews standen die (beruflichen) Sprachlern- und Sprachanwendungserfahrungen der Krankenschwestern/-pfleger sowie ihr Blick auf die Bedeutung kommunikativer Kompetenz in der pflegerischen Arbeit. Darüber hinaus wurden auch die beruflichen und sozialen Rahmenbedingungen, innerhalb derer die Befragten sprachlich handeln, berücksichtigt, da diese ihren (sprachlichen) Handlungsspielraum und ihre Handlungsweisen stark bestimmen und prägen. Mit dieser Berücksichtigung des Kontexts, in dem sich die InterviewpartnerInnen bewegen, wurde auch der Forderung nach einem weitem Zugang zum Untersuchungsfeld Rechnung getragen, wodurch allererst Verantwortlichkeiten im System und Machtmechanismen sichtbar gemacht werden können (s.o.).

Ausgangspunkt für die Reflexion des beruflichen Sprachlernprozesses war eine Beschreibung der ersten Berufserfahrungen der ehemaligen NostrifikantInnen, woraus sich drei große Themenbereiche herauskristallisierten: der Berufseinstieg mit unzureichenden Deutschkenntnissen, die Auseinandersetzung mit dem österreichischen Berufsbild und die Reaktionen des beruflichen Umfelds auf die Befragten. Bereits hier zeigte sich, dass Sprache zwar einen zentralen Faktor für einen erfolgreichen Arbeitsbeginn darstellt, dass jedoch andere Themen (z.B. Einführung in die Arbeit, Aufnahme ins Team, Pflegeverständnis, Umgang mit Statusverlust, Konfrontation mit Ablehnung und Rassismus) ebenso wichtig sind und im Rahmen einer umfassenden kritischen Erhebung, die auf eine Systemveränderung abzielt, auch berücksichtigt werden müssen. Im Folgenden möchte ich mich auf den ersten Themenbereich, den Berufseinstieg, konzentrieren und von dort den Bogen zu einer kritischen Systemeinschätzung spannen.

\section{Sprachbedürfnisse beim Berufseinstieg}

9f $\backslash 2^{\mathrm{vi}}$ : Und die Anfang hab ich sehr schwierig in Station wegen Sprache, aber eine Monat habe ich eigentlich die Arbeit, hab ich alles gelernt, aber Probleme mit Deutsch, Probleme [...], wenn eine Kollegin spricht, verstehe schon, aber ich kann nicht äh, zurücksprechen, weißt du. Es war ein bisschen schwierig, ich haltete zurück eigentlich, weißt, aber nach einem Jahr angefangen ein bisschen sprechen.

Gute Deutschkenntnisse spielen beim Berufseinstieg eine zentrale Rolle. Sie geben gewissermaßen eine Grundsicherheit, die diese generell sensible Phase deutlich erleichtert. Sonia aus Indien, die eingangs zitierte diplomierte Gesundheits- und Krankenschwester, brachte diese Grundvoraussetzung damals nicht mit; sie und drei weitere Personen aus der Stichprobe gehören zu einer Gruppe von Pflegekräften, die Mitte der 90er Jahre binnen kürzester Zeit und zum Teil ohne entspre- 
chende Deutschkenntnisse nostrifiziert hat. Die Folgen sind bei ihr noch immer sichtbar: sie verfügt über ein stark fossiliertes Deutsch, das streckenweise nur sehr schwer verständlich ist, kompensiert diese Defizite jedoch mit einer äußerst freundlichen und fröhlichen Umgangsart. Auch heute werden immer wieder Personen - v.a. PflegehelferInnen, die ihr Gesundheits- und Krankenpflegediplom noch nicht nostrifiziert haben -, die nur über sehr geringe Deutschkenntnisse verfügen, angestellt. Dies führt zu einer schwierigen Arbeitssituation, die nicht nur für die PatientInnen, sondern für das ganze Team als belastend erlebt wird:

5f $\backslash 15$ : Z.B. manche haben Glück und kommen zwischen die Leute, die von seinem Land sind oder so und die schaffen, aber mit Bewohner ist auch sehr schwierig, z.B. sind nicht diese Kollegen jeden Tag da, dass sie übersetzen können. Die sollten schon vorher Deutschprüfung oder einen Kurs besuchen, dass sie bissl Ahnung haben. Nicht so, weil der Bewohner verlangt etwas und dann verstehst du nicht, dann musst du laufen, rennen, rufst du jemanden und „Was sagt der, was will er?“ ist bissl peinlich, bissl unangenehm. Für mich auch, wenn ich so was höre auch, naja, bissl schwierig. Die sollen doch. Aber vielleicht die nehmen solche Leute, durch so wenig Arbeitskraft und die nehmen auch so ohne Deutschkenntnisse, naja, nur dass Kraft da ist, aber das ist nicht alles! Müssen schon bissl auf die Sprache achten.

Die nachstehenden Interviewausschnitte machen deutlich, dass auch Personen mit relativ guten Deutschkenntnissen zu Beginn ihrer Berufstätigkeit in Österreich mit sprachlichen Schwierigkeiten zu kämpfen hatten. Diese bewegen sich auf verschiedenen Ebenen. Einerseits betreffen sie den berufsspezifischen Wortschatz, und hier v.a. das Wissen um die Bezeichnung von Pflegeutensilien:

$1 \mathrm{~m} \backslash 30$ : Ich habe keine Ahnung gehabt und eine von die Schwestern hat mir gesagt, bringst du bitte Waschlabor und ich hab gar nichts verstanden, was ist eine Waschlabor, ja. Weil wir lernen zu Hause etwas anderes. Das ist English eigentlich, die Wort für die Waschlabor kenne ich in English, aber das kenne ich in Deutsch nicht, eigentlich, Waschlabor und die Pflegeutensils. Es gibt verschiedene Namen für die Pflegeutensils, so was wir brauchen zu Hause und hier. Das ist verschiedenes. Statt Waschlabor ich habe Waschlotion genommen. Und hat mir geschimpft und das ist mein erster Tag.

$2 f \backslash 21$ : [denkt] Da gibt's einige [lacht]: Leintuch, das hab ich auch nicht gewusst, was das bedeutet, so ganz einfache Wörter auch. [...] So: Inkueinlage

9f $\backslash 8$ : Ja, manche, Anfang, erste Monate hab ich so nervös geworden, weil ich die, manche versteh, z.B. Konsellarzettel, Konsellarzettel, was ist der Konsellarzettel, eh, Konsellarzettel [Konsiliarschein; Anm.d.Verf.]? Solche Dinge ich muss, ich muss wissen, was ist das English ist das, muss man übersetzen jemand. Das ist sehr wichtig, z.B. nimm das, nimm das, muss alle wissen, ja. Das muss man genau so lernen, was ist der Decke, was ist der Windel, jede Wort muss man wie eine kleine Kind, muss man lernen, was ist der Kugelschreiber, was ist das, was ist das muss jede Wort lernen.

12f $\backslash 25:$ Alle Ausdrücke, z.B. bei Betten machen diese Ausdrücke, ja, Leintuch, das kennt man eh jede, aber für mich war diese Immerstraff, was ich in Deutschland gelernt habe, ich wusste nicht, was ist das. [...] Ja, solche Ausdrücke, was man bei Bet- 
tenmachen und bei der Umgebung, z.B. Nierenschale, Nierentasse oder solchane. Oder Einlauf, was ist ein Einlauf ist? Weil da ham wir auch so [lacht] Schwierigkeiten gehabt, darüber haben wir nur gelacht. Eine unsere Kollegin wusste nicht, was ist das und hat die deutsche Schwester gefragt, kannst du einen Einlauf machen und sie hat gesagt: ja. Sie hat die Patienten umgezogen und mit ihr spazieren gegangen.

Klassische (medizinische) Fachtermini bereiteten hingegen kaum Probleme, da diese aus der eigenen Ausbildung bekannt waren bzw. im pflegerischen Alltag wenig zum Einsatz kommen. Dieses Ergebnis deckt sich mit Beobachtungen in unterschiedlichen beruflichen Kontexten (vgl. z.B. Egbers 1993; Fluck 1992: 144; Müller 1996), die bestätigen, dass der Erwerb spezifischer Fachterminologie oft nicht das Kernproblem für Lernende darstellt, vielmehr fehlt „die Sprache rundherum“, sei es in einfachen Gesprächssituationen oder im Textverständnis:

10f $\backslash 16$ : Medizinische Fachsprache, eigentlich ist kein Problem, weil das ist meistens alles Latein und das lernt man in der Schule eigentlich.

13f $\backslash 26$ : Die Terminologie, nein, nicht so [wichtig; Anm. d.Verf.]. Wir verwenden auch nicht so. [...] Weil die lateinisch brauchen wir eh nicht beim Arbeit, aber die deutsche Wörter schon.

Andererseits betreffen die Schwierigkeiten das Verstehen der PatientInnen und KollegInnen, was auch auf die Verwendung von Dialekt zurückzuführen ist, der von den Betroffenen als große Verstehensbarriere empfunden wird.

6f $\backslash 24$ : „Bist kuma!“ Und ich: Was?! Ich soll Sie kämmen? [lacht] „Bist kuma, is net kuma?" Und „Host a Feierzeig?" Und ich schau nur, was will von mir.

13f $\backslash 20$ : Ja, gemma! Am Anfang hab ich so, was ist gemma? Die manche Patient sagt: „Gemma, Schwester!“ [lacht]

Auch wenn manche Aussagen in diesem Zusammenhang mit einem gewissen $\mathrm{Hu}-$ mor getätigt wurden (s.o.), so empfanden fast alle Befragten ihren ersten Kontakt mit ÖsterreicherInnen als „Schock“, da sie plötzlich mit unbekannten Varietäten konfrontiert waren und - oftmals trotz guter Deutschkenntnisse - große Verstehensschwierigkeiten hatten.

3f $\backslash 10$ : Sag ich Ihnen: Ich bin mit „Guten Tag“ und „Auf Wiedersehen“ gekommen und solchen Grundkenntnissen und damit will ich sagen, hab ich schon verstanden, aber nicht alles. Und wenn die Stationsleiterin im XXX damals die Klosterschwester mich angesprochen hat, bumsti, Gottes Willen: Wienerisch. Hab ich nicht verstanden. Aber dann ist eine Kollegin, ganz junge Schwester, Österreicherin, mit mir durchgegangen. In zwei Wochen habe ich durch Praktikum auf die Station sehr viel verstanden, sehr viel gelernt.

Anna war von der neuen Sprachsituation anfangs gänzlich überfordert und hat erst durch die persönliche Hilfestellung einer Kollegin Zugang zum „Wienerischen“, wie sie es nennt, erhalten. Auch Natascha und Agnes berichten, dass sie einige Zeit brauchten, um sich in den Dialekt bzw. die Umgangssprache in Wien einzuhören 
und sie zu verstehen, da sie sich stark von der erlernten Standardvarietät (im Fall Nataschas (11f) war es - zusätzlich erschwerend - jene Deutschlands) unterscheidet.

11f $\backslash 8$ : Also ich hab ungefähr so eine richtige Hochdeutsch gekonnt, aber der, der Slang, der hier läufig ist, hat mir auch Schwierigkeiten gemacht, also Wienerisch und ich mein jetzt nicht überhaupt allgemein Dialekt, aber überhaupt Wienerisch Deutsch ist ganz anders wie ein richtige Deutsch. Also Deutsch in Deutschland ist so ganz deutlich und hier wird eher so verwaschen gesprochen und das war für mich auch, für mein Ohr, musst ich mich auch ein bisschen einstellen [lacht].

2f $\backslash$ 6: Das ist dann schwierig, weil Dialekt ist bissi schwierig zu verstehen. aber ich hab schon langsam, also ich hab schon angewöhnt sozusagen zu Dialekt auch.

Alle Befragten haben jedoch Verständnis für den Einsatz von Dialekt bzw. Umgangssprache und betonen, dass es wichtig und unumgänglich ist, diese zumindest verstehen zu lernen und, falls dennoch Verstehensprobleme auftauchen, bewusst die Verwendung von Hochdeutsch, also der österreichischen Standardvarietät des Deutschen, einzufordern. Die große Bandbreite an sprachlichen Varietäten erfordern eine hohe Verstehensleistung und Flexibilität, um - etwa im Fall von Verstehensschwierigkeiten - angemessen und den Bedürfnissen der Personen entsprechend reagieren zu können. Auf der Seite der DialektsprecherInnen, und hier vor allem der ArbeitskollegInnen, ist ebenfalls ein hohes Maß an Bewusstsein und Sensibilität in ihrer Sprachverwendung gefragt; Sprache stiftet Identität und schafft Gruppenzugehörigkeit, wodurch Personen, die einer bestimmten Varietät nicht mächtig sind, automatisch ausgeschlossen werden und/oder sich selbst abgrenzen, indem sie sich bewusst nicht auf diese Varietät einlassen möchten.

$1 \mathrm{~m} \backslash 42$ : Wenn wir zusammen in der Küche sitzt und Kaffee trinkt, äh, z.B. [...] ich bin nur drei Jahre in Österreich und ich verstehe ganz wenige Dialekt, äh, wenn die anderen Kollegen, z.B. Österreicherinnen oder Österreicher oder bei uns nur Österreicherinnen, die sitzt, die redet Dialekt, ja, und ich verstehe das. Mit mir redet vielleicht Hochdeutsch, vielleicht, ich verstehe. Aber miteinander, ja, miteinander sie schon redet Dialekt und ich verstehe „boh“. Und ich habe kein Interesse zum das richtig hören und Dialekt wollte ich nicht eigentlich lernen.

$3 f \backslash 13$ : In solchen Anstalten wie xxx ist, kann ich sagen, $80 \%$ von ausländischen Personal die Arbeit abgedeckt ist. Man lernt dort diverse Aussagen oder überhaupt, das ist spezielle deutsche Sprache. Das ist nicht richtig Deutsch.

In diesen Aussagen schwingt auch eine tendenziell negative Bewertung der vorgefundenen Varietäten bzw. Soziolekte mit, die mit einem niedrigeren sozialen Wert oder Prestige konnotiert sind: „Das ist nicht richtig Deutsch“ - gegenüber dem Hochdeutsch, das die meisten in formellen Kontexten erlernt haben. Diese Identifikation und Beurteilung verschiedener sprachlicher Ebenen verlangt bereits ein relativ differenziertes Sprachbewusstsein, dessen Entwicklung auch ein Ziel eines berufsspezifischen Deutschkurses sein müsste: Denn nur durch einen differenzierten Umgang mit verschiedenen sprachlichen Erscheinungsformen und nicht durch 
ihr unreflektiertes Imitieren wird eine situations- und adressatenadäquate Kommunikation möglich.

In den folgenden Stellungnahmen wird über das Verstehen hinaus auch die Unsicherheit im kommunikativen Umgang mit PatientInnen und KollegInnen thematisiert. Dabei spielt das fehlende Wissen um die sozialen und kommunikativen Spielregeln auf einer Station bzw. die Existenz beruflicher Hierarchien eine wesentliche Rolle.

2f $\backslash 18$ : Also ich hab damit am Anfang sehr viele Schwierigkeiten gehabt. Die Stationsschwester hat mir auch gesagt, dass ich, dass die Seiten noch ein bissi, hm, mich entwickeln soll, weil ich muss mehr mit Bewohnern reden. Und das war nicht der Problem, dass ich nicht reden wollte, sondern [lacht], das war schwierig, wirklich. Weil die meisten, meistens Bewohnern sind bissi so ältere Menschen und die reden auch noch mit Dialekt.

7f $\backslash 26$ : Ja, bei Visite ist unangenehm [beide lachen]: „Bitte? Noch einmal?“ Die sagen immer, die sind lieb, ich kann nicht schlecht sagen, aber für mich ist das.

6f: Das war jetzt am Sonntag, wir haben zusammen Dienst gehabt und sollte Arzt kommen und Gordana [7f; d.Verf.]: „Wer geht zum Arzt, wer geht mit ihm?" und dann wir sind aber zu zweit gegangen und das war schon gut, weil wenn ich hab etwas nicht verstanden, hat sie verstanden und wenn sie nicht, dann ich. Und wir haben das zusammengegeben. [...] Aber wir haben schon Angst, weil er ist nicht streng, aber er ist so

7f: Wie sagt man: Respekt wir haben?

11f $\backslash 13$ : Eigentlich kaum [Kontakt mit ÄrztInnen; Anm. B.H.]. Und wenn, dann war nur Anordnung und viele, also Chef z.B. von meiner Abteilung hat geflüstert für sich, den hat ma überhaupt net verstanden, generell jetzt, egal ob Sprache oder nicht, nur Anfang hab ich mich nicht getraut ihm zum fragen, z.B. weil er ist sehr neurotisch gewesen, also, wenn ma nachgfragt hat, ist er explodiert. So später dann, war das für mich, wo ich Stärke und Sprache, dann hab ich nachgfragt und wenn er sich aufgregt hat, dann hamma das halt anders geregelt. Auf jeden Fall musste ich nachfragen, dann hab ich halt gefragt eine Schwester oder einen Arzt oder so. [Herv.d.Verf.]

Natascha (11f) fasst diesen Lernprozess sehr prägnant zusammen: erst als sie „Stärke und Sprache“, d.h. das nötige Selbstvertrauen und (sprachliche) Sicherheit, hatte, konnte sie aktiv am Kommunikationsprozess auf ihrer Station teilnehmen und diesen mitgestalten bzw. in schwierigen Kommunikationssituationen Stellung beziehen.

Ein weiteres Thema, das sich für viele zu Beginn problembehaftet darstellte, war der Umgang mit Humor, v.a. das Verstehen und Reagieren auf Witze, die von PatientInnen und KollegInnen gemacht werden, wobei Witze von Vorgesetzten zusätzlich Unsicherheit erzeugten und zu Vermeidungsstrategien führten:

6f $\backslash 21$ : Ich versteh auch, wenn jemand macht Witz oder so, aber ich weiß nicht, was dazu sagen, aber ich versteh schon. 
13f $\backslash 20$ : Oder manchmal die Oberarzt wollt mich auch bissi sekkieren, er sagt auch manchmal, ich habe mit die Oberarzt sehr gute Kontakt, wir machen immer so viel Spaß, manchmal sagt er auch so [lacht], da versteh ich nicht.

11f $\backslash 12$ : Einmal war, das werde ich nie vergessen, das warn ja urologische Patienten, muss man sagen, sind manchmal ordinär und, und halt die Männer, ja, wiss ma, und wenn eine junge Schwester kommt, die ham halt so Witze gemacht, das war ganz normal. Ja, und der hat mir da gesagt: „Na, hupfst zu mir ins Bett!“ und ich hab das nicht verstanden [lacht] und ich hab gesagt: o.k.! [lacht] Da war das schon blöd! [lacht] Aber das war eigentlich die erste zwei Monate so, dass ich, so, ,ich hab Schmerzen" oder so das Wichtige hab ich verstanden. Es war eher dann schwierig, wenn wer angefangen hat, Witze zu machen oder irgendwie tiefere Kommunikation zu führen, das war in die erste zwei Monate nicht so möglich. [...] \15: Z.B. wenn ein Arzt gekommen ist in Dienstzimmer und wollte Witz erzählen, bin ich lieber rausgegangen, weil Witze zu verstehen hab ich mir noch schwer getan. Aber so alltägliche Kommunikation, die man im Dienst gebraucht hat, hab ich eigentlich beherrscht.

Im Umgang mit diesen sprachlichen Herausforderungen benutzten die Befragten unterschiedliche Strategien: die wichtigsten dabei waren Beobachten und Imitieren sowie Nachfragen, wobei das Nachfragen teilweise von den Befragten abgeblockt wurde oder der Selbstzensur unterworfen war: Man wollte nicht zu viel fragen, um den anderen nicht auf die Nerven zu gehen. Nur drei InterviewpartnerInnen gaben an, systematisch von einer Betreuerin / einem Betreuer angeleitet worden zu sein.

4f $\backslash 15:$ Ja, ja. Ich habe ein bisschen so eine Schwester, die betreut, nicht gerade konkret, aber einfach von jede Kollegin, wenn ich was frage, will ich auch nicht so viel fragen, dass man mich, also wenn viel zu tun ist, dann ist das ein bisschen lästig neue Kollegin.

2f $\backslash 21$ : Also manchmal haben wir keine Zeit, das alles zu erklären, das ist während Arbeit, manchmal muss man sehr flexibel sein und entweder schauen - aha, sie nimmt das, dann wahrscheinlich das ist das. [lacht] Manchmal hat, muss man dann selber darauf kommen, aber nicht immer, wenn ich Zeit habe natürlich, frag ich nach. [...] Aber ich frag nicht immer nach, weil - das find ich unnötig und ich weiß das ganz genau, dass ist auch manchmal ärgerlich, wenn ich jede Minute nachfrage, was ist das, was ist das, was ist das. Also, das ist sicher nicht angenehm. [lacht]

4f $\backslash 24$ : Entweder nachschauen, wie hat die vorher geschrieben. Das sind dann oft die selbe Patient und dieselbe Diagnose, also andere Patienten, aber ähnliche Diagnose: unauffällig, gut geschlafen, ohne Schmerzen, bei Bewusstsein [...] Oder fragen. Aber wenn man selber noch Schreibfehler macht, das liegt dann schon an mich, da muss ich aufpassen. Wörterbuch anschauen.

11f $\backslash 10$ : An und für sich sehr wenig [Einweisung; Anm. B.H.], da war ich sehr unzufrieden. Sehr wenig! Ich bin richtig in wie ein Radl läuft, in dem Radl musste ich einpassen und ich musste alles dafür machen, dass ich reinpass und keine Schwierigkeiten hab. Eigentlich Einführung, Erklärung Operationen, System, wie das abläuft, nichts! Überhaupt nichts! Du bist hergekommen und du musst dich anpassen, so ungefähr war das. Sehr, sehr hart, eigentlich. 
Die Aussagen der interviewten diplomierten Gesundheits- und Krankenschwestern/-pfleger zeigen, dass die Unterstützung beim Berufseinstieg und dem damit verbundenen sprachlichen Lernprozess (z.B. Einführung in die Dokumentation, Ablaufklärung einer Dienstübergabe, etc.) nicht systematisch und flächendeckend erfolgt beziehungsweise überhaupt nicht vorhanden ist. Die Betroffenen sind hier einerseits vom Entgegenkommen der jeweiligen Teammitglieder abhängig und andererseits liegt es in ihrer Eigenverantwortung, sich die erforderlichen Kenntnisse anzueignen. Sie werden dabei mit einem System konfrontiert, das auf ihre (sprachlichen) Bedürfnisse als BerufseinsteigerInnen wenig bis keine Rücksicht nimmt. Umgekehrt wird auch eine Mehrbelastung des Kollegiums durch die erforderlichen Hilfestellungen und Einweisungen deutlich. Ein berufsvorbereitendes oder -begleitendes berufsspezifisches Deutschkursangebot, das auf die hier erhobenen (sprachlichen) Anforderungen und Probleme Bezug nimmt, könnte hier zum einen zu einer großen Entlastung des gesamten Personals beitragen und zum anderen den Betroffenen die notwendige Sicherheit und „Stärke“ geben, sich von Anfang an in ihrem neuen beruflichen Handlungsfeld gut zurechtzufinden, dieses aktiv mitzugestalten und Aufstiegschancen zu entwickeln.

\section{Kritische Einschätzung des Umgangs mit Sprachkenntnis- sen in der Pflege und Veränderungspotential}

Das Gelingen des (sprachlichen) Anpassungsprozesses ist - wie die Erhebungen der sprachlichen Bedürfnisse zeigten - zum Großteil den Betroffenen selbst überantwortet bzw. wird erwartet, dass die fehlenden berufsspezifischen Sprachkenntnisse und auch das Wissen über professionelle Gesprächsführung automatisch in der Praxis erworben werden. Meine Untersuchung hat in Bezug auf die Effizienz dieses Systems durchaus Widersprüchliches zu Tage gefördert: Die Interviews haben, wie auch an den Transkripten zu erkennen ist, zum Teil gravierende sprachliche Schwierigkeiten der Befragten sichtbar gemacht, die auch während der Interviews immer wieder zu Verstehensproblemen geführt haben. Dennoch sind alle befragten Pflegekräfte - zum Teil schon jahrelang - erfolgreich in der Pflege tätig, d.h. sie erfüllen ihre Berufspflichten, arbeiten in und mit einem Team zusammen, halten sich an vorgegebene Pflegestandards, dokumentieren ihre Arbeit etc. Hier drängt sich natürlich die Frage auf, wie das mit den vorhandenen „mangelhaften“ Sprachkenntnissen möglich ist und - nachdem es ganz offensichtlich möglich ist warum es dann überhaupt die von mir geforderte berufsspezifische Sprachförderung geben sollte: Die gängige Praxis, adäquate Sprachkenntnisse direkt in der Berufspraxis selbst - durch learning by doing - zu erwerben, „funktioniert" doch und kommt zudem billiger. Ich möchte exemplarisch, anhand von einer in vielerlei Hinsicht typischen Sprachlern- und Berufsbiographie einer Interviewteilnehmerin, einen Erklärungsversuch unternehmen und diesen - vermeintlichen - Widerspruch auflösen. 
Sonia (9f) ist ohne Deutschkenntnisse nach Österreich gekommen und hat im Zuge des großen Personalmangels Anfang der 90er Jahre - nach einem kurzen Deutschkurs - sehr rasch nostrifiziert. In weiterer Folge hat sie aufgrund von fehlenden Angeboten und Zeitmangel keine weiteren Deutschkurse mehr besucht, sondern hat die notwendigen Sprachkenntnisse ungesteuert direkt im Beruf erworben. Ihr mündliches Deutsch zeichnet sich durch starke Fossilierung aus, schriftlich fallen weniger Schwächen auf. Trotz ihrer unzureichenden mündlichen Kommunikationskompetenz ist sie gut in das Team integriert, erledigt ohne Probleme ihre Aufgaben und pflegt einen äußerst freundlichen Umgang mit den Patienten. Sie ist erfolgreich in ihrem Beruf tätig, obwohl man von ihr sagen würde, dass sie „schlechte Deutschkenntnisse“ hat.

Sonia betont, dass sie in einem sehr guten Team arbeite und dass die Stationsschwester darauf bedacht sei, alle nach ihren besonderen Interessen und Fähigkeiten einzusetzen. Das bedeutet für sie, dass sie sich v.a. um medizinische Tätigkeiten (z.B. Medikamente einschachteln, Infusionen geben etc.) kümmern kann, die wenig Kommunikation voraussetzen:

9f $\backslash 17$ : Z.B. unsere Stationsschwester ist sehr gute Stationsschwester, sie, sie nimmt jede, z.B. jede hat eigene, z.B. ich interessiere mehr für medizinische Sachen, ja, manche interessieren baden gerne, die gehen diese Richtung, ja, z.B. ich gerne mit medizinische Richtung, ich habe mehr dort, meine Land mit medizinische Richtung gelernt, dann gehe ich, nehme Medikamentenwagen, nehme ich meine Spritze und solche Infusion und solche Behandlung. [...] Dann funktioniert schon gut, eigentlich, ja.

Bei der Dokumentation kommt ihr einerseits entgegen, dass sich in der Langzeitpflege Pflegehandlungen stark wiederholen und andererseits hält sie selbst ihre Einträge sehr knapp.

9f $\backslash 11$ : Das ist, Anfang schreiben nicht so viel: Patient wurde gebadet, nur kurze, kurze, kurze Worte, ja, das ist nicht viel geschrieben, jetzt fangt schon so viele Dokumentation und so, alles eintragen, ja was wichtig ist, schreiben schon, das geht, ja, aber nicht so viel, Roman kann ich nicht [lacht].

Die hohe Routinisierung und Ritualisierung der Arbeit dürfte Sonia auch in der mündlichen Kommunikation mit den Patienten - hier handelt es sich um eine reine Männerstation mit z.T. schwer dementen Patienten - entlasten. Die Gesprächsanlässe und -themen sind - entlang der immer relativ gleich bleibenden Tagesstruktur mit fix vorgegebenen Aufgaben - meist die gleichen und stellen nach einer gewissen Zeit keine große Herausforderung dar. Zudem kompensiert sie hier ihre Schwächen mit nonverbaler Kommunikation sowie sehr hoher sozialer Kompetenz und Empathie.

Ein eingespieltes Team, gleichförmige Tagesabläufe und Kompensationsstrategien ermöglichen Sonia somit ein erfolgreiches Handeln in ihrem Beruf. Sie selbst weiß, wo ihre Schwächen liegen und würde sich auch gerne im Bereich der mündlichen Kommunikation verbessern, wenn es ein entsprechendes Angebot gäbe. Allerdings war bisher auch keine dringende Notwendigkeit gegeben, sich sprach- 
lich zu verbessern; die kommunikativen Herausforderungen in der Altenpflege haben bei ihr keine dringenden Sprachbedürfnisse ausgelöst oder Impulse zur Weiterentwicklung ihrer Berufssprache gesetzt.

Sonia kann auf kommunikativer Ebene eine Routinepflege oder sog. notwendige Pflege (vgl. Korečić 2005)vii, die dem pflegerischen Minimalstandard entspricht, durchführen. Für eine angemessene oder optimale Pflege fehlen ihr grundlegende kommunikative Kompetenzen, um etwa ein reaktivierendes Gespräch nach dem psychobiographischen Pflegemodell (vgl. Böhm 2001) oder ein Beratungsgespräch mit Angehörigen zu führen. Auch im schriftlichen Bereich ist es ihr nicht möglich, über dem Minimalstandard zu arbeiten. Sie verwendet in der Dokumentation kurze formelhafte Sätze, mittels derer sie komplexere Beobachtungen oder Pflegeplanungen jedoch nicht ausdrücken könnte, was an ihrem Arbeitsplatz aber auch nicht gefordert ist.

Sonias sprachliche Mängel, die nach Hutchinson und Waters (1987) die Differenz zwischen Sprachstand und sprachlichen Anforderungen in der Zielsituation darstellen, sind in ihrer jetzigen Arbeitssituation in der Altenpflege demnach relativ gering. Würde man sie an den theoretischen Anforderungen aus der Pflegeliteratur, die eine optimale Pflege anstreben, messen, wäre die Differenz hingegen enorm und ihre berufliche Handlungsfähigkeit plötzlich drastisch eingeschränkt.

Hier lässt sich ein eindeutiger Zusammenhang zwischen kommunikativer Kompetenz und Pflegequalität herstellen: Mit steigender Pflegequalität steigen auch die kommunikativen Anforderungen im jeweiligen Arbeitsfeld. Besonders offenkundig wird dies bei der Personalpolitik von Privatspitälern, die mit einer besonders hohen Pflegequalität werben. Laut Berger würden hier nur Leute mit exzellenten Sprachkenntnissen genommen, da es sich Privatspitäler nicht leisten könnten, (sprachlich) mangelhaft qualifiziertes Personal anzustellen. viii Auch Ford betont, dass Sprache ein wichtiger Bestandteil der Qualitätssicherung in der Pflege (vgl. Ford 1997) ist. Die Umsetzung des Pflegeprozesses, der hohe sprachliche Kompetenzen erfordert, ist beispielsweise ein zentraler Faktor der Qualitätsentwicklung in der gesamten Pflege. Wenn er aufgrund von sprachlichen Defiziten nicht adäquat umgesetzt werden kann, so hat dies negative Folgen für die Qualität der Pflege und kann im schlimmsten Fall ,gleichzeitig eine Gesundheitsgefahr für die Patienten und ein betriebswirtschaftliches Risiko für die Gesundheitseinrichtung" (Ford 1997: 171) darstellen. Vor allem in der Altenpflege wird dieses Risiko jedoch offenbar in Kauf genommen bzw. gering eingeschätzt.

Der Großteil der zugewanderten Pflegekräfte beginnt ihre Tätigkeit - oftmals mit mangelnden Deutschkenntnissen - in diesem Bereich, der innerhalb der Pflege für die Befragten jedoch das mit Abstand unattraktivste Arbeitsfeld darstellt, was einerseits an der physisch und psychisch besonders belastenden Arbeitssituation, aber auch am niedrigen Sozialprestige dieses Bereichs liegt. Zudem fühlten sich die meisten InterviewpartnerInnen für diese Arbeit, die sie mit „waschen, füttern, wickeln“ zusammenfassen, überqualifiziert. Tatjana bringt dies im Interview folgendermaßen auf den Punkt: 
6f $\backslash 30$ : Und wozu haben wir diese Nostrifikation, wenn ich muss im Pflegeheim arbeiten? Das ist gut für Anfang, diese Pflegeheim, das ist klar, und das wissen alle, nur niemand das sagt.

Fast alle Befragten möchten daher den Arbeitsplatz wechseln und einen Job im Spital finden. Voraussetzung dafür sind - nach ihren eigenen Aussagen - bessere Deutschkenntnisse aufgrund höherer Verantwortung. Man könnte in diesem Zusammenhang dem System auch fehlendes Interesse unterstellen, die Leute sprachlich weiterzubilden, um sie in der Langzeitpflege zu halten. - In meiner Stichprobe gab es hier übrigens eine eindeutige Korrelation zwischen dem Sprachstand und Karriereweg. Die Befragten mit ausgezeichneten Deutschkenntnissen arbeiteten mit einer Ausnahme entweder im Spital oder selbständig oder waren nicht mehr in der Pflege tätig.

Die Bedeutung, die kommunikativer Kompetenz in der Langzeit- bzw. Altenpflege zugemessen wird, kann hier auch als Indikator dafür gelten, wie viel die Pflege alter Menschen wert ist: Routinepflege „mit wenig Worten“ scheint hier ausreichend $\mathrm{zu}$ sein. Es muss davon ausgegangen werden, dass Koch-Straubes Aussage über das Pflegeheim als einen „Ort verkürzter Sprache oder des Verstummens" (Koch-Straube 1997: 11) auch heute noch in vielen Fällen zutrifft, wobei es sich hier um einen Circulus vitiosus handelt: Die Mängel im System (Personalmangel, Zeitdruck, Routinepflege, mangelndes Prestige) ermöglichen und verlangen einerseits den Einsatz von Pflegepersonal auch mit unzureichenden Deutschkenntnissen, gleichzeitig prolongiert dieser aber wieder einen Teil dieser Mängel. Möchte man eine höhere Qualität in der Pflege erreichen, die auf einem ganzheitlichen, auf die Bedürfnisse der einzelnen PatientInnen ausgerichteten Pflegeverständnis beruht, so müssen diese Rahmenbedingungen kritisch analysiert und verändert werden. In diesem Zusammenhang wird es auch notwendig sein zu fragen, ob ein Verzicht von offizieller Seite auf ein berufsspezifisches Sprachlernangebot sowie eine einheitlich geregelte Einstiegsphase (z.B. in Form eines Mentoring-Systems direkt am Arbeitsplatz) für zugewandertes Pflegepersonal heute vor dem Hintergrund steigender Qualitätsansprüche und Anforderungen in der Pflege noch leistbar ist. Der Status quo im Umgang mit sprachlicher Kompetenz - der sich mit dem Slogan „Forderung ohne Förderung“ zusammenfassen lässt -, wirft somit ein Schlaglicht auf die Gesamtsituation in der Pflege.

Eine hohe Qualität, die in einem Beruf wie der Gesundheits- und Krankenpflege ohne hohe kommunikative Kompetenzen der Pflegekräfte aufgrund zunehmender Professionalisierung nicht mehr erreichbar ist, ist zweifelsohne von allgemeinem Interesse, sowohl aus wirtschaftlicher als auch aus humaner Sicht. Demnach gehört es in die öffentliche Verantwortung, für dringend benötigte Arbeitskräfte ein Angebot zu schaffen, das ihnen einen guten und sicheren Berufseinstieg, höhere Berufszufriedenheit sowie eine Weiterentwicklung in diesem Beruf durch aktive und kompetente Mitgestaltung ermöglicht und gleichzeitig eine hohe Pflegequalität für PatientInnen und KlientInnen garantiert. 


\section{Literatur}

Abt-Zegelin, Angelika \& Schnell, Martin W. (Hrsg.) (2006): Die Sprachen der Pflege. Interdisæiplinäre Beiträge aus Pflegewissenschaft, Mediæin, Linguistik und Philosophie. Hannover: Schlüttersche Verlagsgesellschaft [Wittener Schriften].

Arndt, Peter S. (1998): Sprachbedarfsanalyse für berufsorientierten Deutschunterricht. Bildungsarbeit in der Zweitsprache Deutsch 1, 26-32.

Basturkmen, Helen (2002): Towards a framework for analyzing theory and practice in LSP. In: IRAL. International Review of Applied Linguistics in Language Teaching. 40(1), 23-35.

Benesch, Sarah (1993): ESL, Ideology, and the Politics of Pragmatism. In: TESOL Quarterly $4,705-716$.

Benesch, Sarah (1996): Needs Analysis and Curriculum Development in EAP: An Example of a Critical Approach. In: TESOL Quarterly 4, 723-738.

Benesch, Sarah (1999): Thinking Critically, Thinking Dialogically. In: TESOL Quarterly 3, 573-580.

Böhm, Erwin (2001): Psychobiographisches Pflegemodell nach Böhm, Bd. 1: Grundlagen; Bd. 2: Arbeitsbuch. Wien: Maudrich.

Canagarajah, A. Suresh (1993): Critical Ethnography of a Sri Lankan Classroom: Ambiguities in Student Opposition to Reproduction throught ESOL. In: TESOL Quarterly 4, 601-626.

Darmann, Ingrid (2000): Kommunikative Kompetenz in der Pflege. Ein pflegedidaktisches Konzept auf der Basis einer qualitativen Analyse der pflegerischen Kommunikation. Stuttgart [u.a.]:

Kohlhammer.

Dudley-Evans, Tony; \& St John, Maggie Jo (1998): Developments in ESP. A multi-disciplinary approach. Cambridge: CUP.

Durrant, Cal \& Green, Bill (2000): Literacy and the new technologies in school education: Meeting the 1(IT)eracy challenge? In: The Australian Journal of Language and Literacy 23(2), 89-108.

Dymarczyk, Christina (2003): Das Altenheim aus Perspektive des Pflegepersonals - Lebensort für die eigene Zukunft? Empirische Studie zur Verbesserung der Lebensbedingungen in der stationären Altenpflege. Dissertation: Bonn. [http://www.ilr1.uni-bonn.de/wiso/DissDipl.htm; 20.09.2008]

Egbers, Ursula (1993): Überlegungen zum Fachsprachenunterricht in einer Berufsvorbereitungsklasse für Ausländer (BVKA) in der Fachrichtung „Hauswirtschaft und Pflege" - dargestellt am Bereich Gesundheitspflege. In: Bildungsarbeit in der Zweitsprache Deutsch 1, 27-35.

Friebe, Jens (2006): MigA - Migrantinnen und Migranten in der Altenpflege. Bestandsaufnahme, Personalgewinnung und Qualifizierung in Nordrbein-Westfalen. Eine Handreichung für Bildung und Praxis in der Altenpflege. Bonn: Deutsches Institut für Erwachsenenbildung. [http://www.diebonn.de/publikationen/online-texte/details.asp?ID=3535; 20.09.2008] 
Fluck, Hans-Rüdiger (1992): Didaktik der Fachsprachen. Aufgaben und Arbeitsfelder, Konzepte und Perspektiven im Sprachbereich Deutsch. Tübingen: Narr [Forum für FachsprachenForschung, 16].

Ford, Yvonne (1997): Workshop „Sprachliche Implikationen eines multikulturellen Pflegeteams“. In: Zegelin (Hrsg.), 167-172.

Fthenakis, Wassilios E.; Sonner, Adelheid; Thrul, Rosemarie \& Walbiner, Waltraud (1985): Bilingual-bikulturelle Entwicklung des Kindes. Ein Handbuch für Psychologen, Pädagogen und Linguisten. Hrsg.vom Staatsinstitut für Frühpädagogik. München: Max Hueber.

Funk, Hermann (1992): Berufsbezogener Deutschunterricht. Grundlagen - Lernziele Aufgaben. In: Fremdsprache Deutsch, Sondernummer 1992, 4-16.

Green, Bill (1988): Subject-specific Literacy and School Learning: A Focus on Writing. In: Australian Journal of Education, 32(2), 156-179.

Grünhage-Monetti, Matilde (Hrsg.) (2000): Odysseus - Zweitsprache am Arbeitsplat: Sprachbedarfe und-bedürfnisse von ArbeitsmigrantInnen: Konzepte des Sprachelernens im berufsbezogenen Kontext (Projekt 1.2.5). Graz: European Centre for Modern Languages.

Grünhage-Monetti, Matilde; Halewijn, Elwine \& Holland, Chris (Hrsg.) (2003): Odysseus Second language at the workplace. Language needs of migrant workers: organising, language learning for the vocational/ workplace context. European Centre for Modern Languages. Strasbourg: Council of Europe Publishing. [incl. CD-Rom]

Grünhage-Monetti, Matilde \& Klepp, Andreas (2004): Zweitsprache am Arbeitsplatz als Herausforderung für Integration und Partizipation. Europäische Perspektiven. In: Deutsch als Zweitsprache 1, S. 15-20.

GuKG (1997) = Gesundheits- und Krankenpflegegesetz; Weiss-Faßbinder, Susanne \& Lust, Alexandra (Hrsg.) (2004).

Haider, Barbara (2003): Na, wie geht es Ihnen heute? Deutsch für gesundheitsorientierte Berufe an einer Wiener Volkshochschule. In: ÖDaF-Mitteilungen. Informationen des Vereins „Österreichischer Verband für Deutsch als Fremdsprache / Zweitsprache"2, 43-50.

Haider, Barbara (2004): Deutsch für Pflegepersonen nicht-deutscher Muttersprache. In: Österreichische Pflegezeitschrift. Zeitschrift des Österreichischen Gesundheits- und Krankenpflegeverbandes 3, 32-34.

Haider, Barbara \& Laimer, Thomas (2006): Deutsch für Gesundheits- und Krankenpflege. Materialienmappe. Wien: Österreich Institut [Materialienreihe Fachsprache Deutsch].

Holliday, Adrian (1995): Assessing Language Needs Within an Institutional Context: An Ethnographic Approach. In: English for Specific Purposes 2, 115-126.

Hutchinson, Tom \& Waters, Alan (1987): English for Specific Purposes. A learning-centred approach. Cambridge: CUP [New Directions in Language Teaching].

Jasso-Aguilar, Rebeca (2001): Sources, Methods and Triangulation in Needs Analysis: A Critical Perspective in a Case Study of Waikiki Hotel Maids. In: English for Specific Purposes 1, 27-48. 
Juchli, Liliane (71994): Pflege. Praxis und Theorie der Gesundheits- und Krankenpflege.

Stuttgart/New York: Georg Thieme.

Koch-Straube, Ursula (1997): Fremde Welt Pflegeheim. Eine ethnologische Studie. Bern [u.a.]: Hans Huber.

Korečić, Jasenka (42005): Pflegestandards Altenpflege. Heidelberg: Springer.

Krajic, Karl \& Nowak, Peter (2003): Pflegenotstand in Österreich? Diagnosen und Lösungsmöglichkeiten mit einem Schwerpunkt auf Entwicklung der Arbeitsbedingungen des diplomierten Pflegepersonals. Wissenschaftliches Gutachten des Ludwig Boltzmann Instituts für Medizin- und Gesundheitssoziologie: Wien.

Müller, Annette (1996): Förderkonzept „Deutsch als Zweitsprache“ im ausbildungsbegleitenden Deutschunterricht. In: Bildungsarbeit in der Zweitsprache Deutsch 3, 13-20.

Nispel, Andrea \& Szablewski-Çavuş, Petra (1997): Über Hürden, über Brücken. Berufliche Weiterbildung mit Migrantinnen und Migranten. Frankfurt am Main: DIE.

Nispel, Andrea \& Szablewski-Çavuş, Petra (Hrsg.) (1996): Lernen, verständigen, handeln. Berufsbezogenes Deutsch. Frankfurt am Main: DIE [Praxishilfen berufliche Weiterbildung mit Erwachsenen ausländischer Herkunft; 4].

Nunan, David (2004): Task-Based Language Teaching. Cambridge: CUP [Cambridge Language Teaching Library].

Oertle Bürki, Cornelia (1994): Fachsprachliche Aspekte der Sprache der (Kranken-)Pflege. Hrsg. v. der Abteilung Berufsbildung SRK Bern [Hefte Bereich Berufsbildung; 1].

Richterich, René (Hg.) (1983): Case Studies in Identifying Language Needs. Prepared for the Council of Europe Modern Languages Project. Oxford [u.a.]: Pergamon Press.

Robinson, Pauline C. (1991): ESP Today. A Practioner's Guide. New York [u.a.]: Prentice Hall.

Sachweh, Svenja (2002): „Noch ein Löffelchen?": Effektive Kommunikation in der Altenpflege. Bern [u.a.]: Hans Huber.

Szablewski-Çavuş, Petra (1997): Verstehen und Verständigung: Grundzüge einer berufsbereichsübergreifenden Didaktik. In: Nispel \& Szablewski-Çavuş (Hrsg.), 87-110.

Szablewski-Çavuş, Petra (2000): Verstehen und Verständigung in Deutsch. Grundzüge einer berufsbereichsübergreifenden Didaktik. In: Bildungsarbeit in der Zweitsprache Deutsch 2, 16-30.

Von der Handt, Gerhard \& Stricker, Heinrich (Hrsg.) (1995): Das Zertifikeat Deutsch für den Beruf. Lernziele, Wortliste, Testmodell, Bewertungskriterien. Hrsg. v. Deutschen Volkshochschulverband (DVV) e.V. und dem Goethe-Institut zur Pflege der deutschen Sprache im Ausland und zur Förderung der internationalen kulturellen Zusammenarbeit e.V.

Walther, Sabine (2003): Sprache und Kommunikation in der Pflege. Forschungsarbeiten und Publikationen zur mündlichen und schriftlichen Kommunikation in der Pflege. Eine kommentierte Bibliographie. Duisburg: Gilles \& Francke. 
Weinhold, Christine (1997): Kommunikation zwischen Patienten und Pflegepersonal. Eine

Gesprächsanalytische Untersuchung des sprachlichen Verbaltens in einem Krankenhaus. Bern [u.a.]: Hans Huber.

Weiss-Faßbinder, Susanne \& Lust, Alexandra (Hrsg.) (2004): Gesundheits- und Krankenpflegegesetz - GuKG samt ausführlichen Erläuterungen. Wien: Manz.

Zegelin, Angelika (Hrsg.) (1997): Sprache und Pflege. Berlin, Wiesbaden: Ullstein Mosby.

i Der Titel der Dissertation lautet „Deutsch für Gesundheits- und Krankenpflegepersonal. Eine kritische Sprachbedarfserhebung vor dem Hintergrund der Nostrifikation von ausländischen Pflegediplomen in Österreich“ (Universität Wien, April 2008). Das Eingangszitat stammt aus einem qualitativen Interview mit Natascha (Name geändert), einer slowakischen Krankenschwester, die 1994 nach Österreich gekommen ist und im gleichen Jahr ihr Gesundheits- und Krankenpflegediplom nostrifiziert, d.h. eine offizielle Anerkennung ihrer Ausbildung erhalten hat (s. Übersichtstabelle im Anhang).

ii $\quad$ LSP $=$ Language for specific purposes (Sprache für spezielle Zwecke).

iii Im Rahmen der Nostrifikation sind Ergänzungsprüfungen in verschiedenen Fächern (z.B. Pharmakologie, Berufsspezifische Rechtsgrundlagen, Allgemeine Gesundheits- und Krankenpflege) abzulegen. Eine Vorbereitung auf diese Prüfungen erfolgt in sog. Nostrifikationslehrgängen. In Wien werden derzeit an zwei Schulen eigene Lehrgänge dieser Art angeboten.

iv Gelegentlich findet sich auch der Terminus subjektiver Sprachbedarf (z.B. Szablewski-Çavuş 1997: 104), der in der Bedeutung von Spracbbedürfnis (s.o.) verwendet wird.

v Nostrifikationen betreffen Personen, die eine Ausbildung außerhalb des EWR-Raums abgeschlossen haben. EWR-BürgerInnen erhalten eine so genannte Berufszulassung, die bei harmonisierten Ausbildungen nur einen Formalakt darstellt, während Nostrifikationen im Allgemeinen mit Ergänzungsprüfungen verbunden sind. Für die neuen EU-Mitgliedsstaaten gibt es in diesem Bereich unterschiedlich lange Übergangsfristen. Vgl.: http://www.wequam.at (Stand: 20.09.2008).

vi Die erste Zahl bezeichnet den/die Interviewpartner/in samt Angabe des Geschlechts (m./f.) (s. Übersichtstabelle im Anhang), die zweite Zahl bezieht sich auf den jeweiligen Abschnitt im Transkript.

vii Nach Korečić (2005) können vier Qualitätsstufen für Pflegequalität unterschieden werden: optimale Pflege (ideal, erstklassig), angemessene Pflege (gut), notwendige Pflege (ausreichend) und mangelhafte Pflege (gefährlich, unzureichend) (ebd.: 40ff.). Die höchste Qualitätsstufe der optimalen Pflege sollte dabei immer als richtungweisend gelten.

viii Die Informationen stammen aus einem Interview mit der Leiterin des Nostrifikationslehrgangs am Allgemeinen Krankenhaus der Stadt Wien, DGKS Elfriede Berger, vom 3.7.2007. 



\title{
Kulturspezifische Einflüsse der Lehr- und Lernumgebung auf die (Aussprache)leistungen chinesischer Deutschlernender
}

\author{
Cordula Hunold (Göttingen)
}

Außersprachliche Faktoren spielen beim Fremdsprachenlernen eine nicht zu unterschätzende Rolle. In Arbeiten zu Lernstrategien oder über autonomes Lernen wurde dabei asiatischen Lernenden auch schon Aufmerksamkeit geschenkt. Aber auch für die Ausspracheschulung ergeben sich kulturspezifische Lehr- und Lernweisen aus einem Faktorenkomplex von soziopolitischen und soziokulturellen sowie personenbezogenen Faktoren in einem bestimmten akademischen Kontext. Diese Faktoren bestimmen auf verschiedene Weise das Curriculum und damit die Prüfungs- und Unterrichtspraxis, aber auch Motive und Motivationen jedes einzelnen Lernenden und Lehrenden. Gerade im Zusammenhang mit dem Chinesischen als Sprache und chinesischen Lernern als Zielgruppe dürfte eine jede Analyse, die die kulturellen Unterschiede zwischen dem Chinesischen und dem Deutschen nicht bewusst macht, ein unvollständiges und fragwürdiges Unterfangen bleiben. Im Folgenden sollen einige dieser Faktoren angesprochen werden und auf ihren Einfluss auf die Leistungen - speziell chinesischer Deutschlernender - hin untersucht werden. Ausgehend von der generellen Situation chinesischer Deutschlernender besonders beim Deutschlernen in China, aber auch in Deutschland, soll der Fokus auf Einflüssen auf die Ausspracheleistungen liegen. Die Ausführungen basieren zunächst auf der Auswertung der Forschungsliteratur zu chinesischen Lernenden und schließen eigene Untersuchungen (Fragebögen, Sprechwirkungsanalysen) ein.

\section{Einleitung - einige Zahlen vorweg}

Zur Situation in Deutschland: In den deutschen Universitäten ist der Anteil chinesischer Studierender in den letzten ca. 30 Jahren kontinuierlich gestiegen. Chinesische Studierende bilden heute bei weitem die größte Gruppe ausländischer Studierender; ihr Anteil an der Gesamtzahl ausländischer Studierender (248 357) beträgt dabei ca. $12 \%$. Andere ostasiatische Studierende bilden gegenüber chinesischen Studierenden eher kleinere Gruppen (Wintersemester 2005/2006: Studierende aus der Republik Korea: 5096; Japan: 2339; Vietnam: 2982). Chinesische Studierende sind dabei in allen Fachbereichen zu finden, jedoch befinden sich überdurchschnittlich viele in ingenieurwissenschaftlichen, rechts-, wirtschafts- und sozialwissenschaftlichen Studiengängen. 


\begin{tabular}{|c|r|r|r|r|r|r|}
\hline & $1980 / 81$ & $1985 / 86$ & $1990 / 91$ & $1995 / 96$ & $2000 / 01$ & $2005 / 6$ \\
\hline VR China & 317 & 1117 & 4230 & 5425 & 9109 & 27390 \\
\hline Taiwan & 475 & 583 & 942 & 1433 & 1265 & 1302 \\
\hline
\end{tabular}

Tabelle 1: Chinesische Studierende aus der VR China und aus Taiwan in Deutschland vom WS 1980/81 bis WS 2005/06 (bis einschl. WS 1990/91 früheres Bundesgebiet, seit 1992/93 gesamtdeutsches Gebiet) (Zahlen bis 2000/01 zit. nach Zeilinger 2006) (Zahlen für 2005/2006 Statistisches Bundesamt 2006).

Zur Situation in China: Mit der 1972 eingeleiteten Öffnungspolitik in China, die zu einer Wiederbelebung und Neugründung von Deutsch- und Germanistikabteilungen in vielen Landesteilen führte (vgl. Fluck 2004), entwickelte sich das Bildungssystem seit 1978 in eine neue Richtung, in deren Folge auch der Fremdsprachenunterricht reformiert wurde. Seit Ende der 1980er Jahre gibt es vier Arten von Deutschunterricht bzw. Germanistikstudium in China: das ,klassische“ Germanistikstudium, Intensiv- und Aufbaukurse für Wissenschaftler anderer Fakultäten, Deutsch als 2. Fremdsprache für Studierende aller Fachrichtungen und Deutschunterricht in Betrieben und Firmen, Abendschulen bzw. Selbstlernprogramme (Kleppin 1987: 252f.). Dies sind nach wie vor die wichtigsten Formen, in denen Deutsch vermittelt wird, wenngleich sich die Qualität und Quantität sowie die Anteile der einzelnen Formen an der Gesamtzahl der Deutschlernprogramme stark gewandelt haben dürften.

Laut Statistik zur Lage der deutschen Sprache im Ausland von der „Ständigen Arbeitsgruppe Deutsch als Fremdsprache“ (StADaF: 2000) war zwar die Anzahl der Germanistik-Studierenden und auch die Zahl der universitären Deutschkurse in den Jahren 1995 - 2000 gesunken, hingegen stach ein enormer Anstieg an DaFSprachkursen und Sprachkursteilnehmern außerhalb universitären Kontextes ins Auge, was für ein nach wie vor starkes Interesse am Deutschlernen - vermutlich vorzugsweise um in Deutschland ein Studium aufzunehmen - spricht. Im Jahr 2000 haben beispielsweise 3790 Teilnehmer im Goethe-Institut Beijing an Sprachkursen teilgenommen. Ein immer wichtiger werdender Bereich ist auch Deutsch als Fremdsprache an (Mittel)Schulen. Im Bericht des DAAD für das Jahr 2005 ist demgegenüber wieder von einem Anstieg der Anzahl der chinesischen Studierenden, die Deutsch in einem vierjährigen Bachelor-Studium als Hauptfach wählen, die Rede. Der Bericht geht von derzeit jährlich 1500 Studienanfängern und insgesamt 6000 Studierenden in DaF-Studiengängen aus (DAAD 2005). Die meisten Deutsch-Studienangebote beziehen sich auf Bachelor-Studiengänge. Es gibt darüber hinaus ca. 20 Germanistik-Masterstudiengänge mit insgesamt jährlich ca. 80 Studienanfängern. Eine Promotion im Fach Germanistik ist an drei Universitäten möglich. Insgesamt geht die DAAD-Studie (2005) von einer Anzahl von ca. 180 Hochschulen aus, an denen Deutsch unterrichtet wird. Deutsch als 2. Fremdsprache lernten 2005 ca. 5500 Studierende, wobei über die Jahre 2002 - 2005 ein An- 
stieg von $77 \%$ zu verzeichnen war (DAAD 2005). Die Studie nennt die wachsende Präsenz deutscher Wirtschaftsunternehmen, das weiterhin große Interesse an einem Auslandsstudium - noch dazu bisher z. T. ohne Studiengebühren - und die derzeitige pro-europäische Politik der chinesischen Regierung als Gründe für das nach wie vor starke Interesse am Deutschlernen bzw. an einem Studium in Deutschland.

\section{Kulturspezifische Lehr- und Lernweisen}

Seit Mitte der 1980er Jahre sind im Rahmen des stetig anwachsenden fachlichen und bildungspolitischen Austausches zwischen Deutschland (und anderen Ländern) und China zahlreiche Fremdsprachenlektorinnen und -lektoren in die Volksrepublik China (und umgekehrt) vermittelt worden. Seit dieser Zeit beschäftigt sich auch die Forschungsliteratur mit den Besonderheiten chinesischer Lehrender und Lernender. Dabei wird sowohl deren chinesische „Ausgangs“-Lernumgebung als auch die nicht-chinesische Lernumgebung beschrieben, in die sich chinesische Studierende begeben, wenn sie im Ausland studieren. Kirby et al. (1996: 141) beschreiben im Einzelnen, dass es einen Unterschied mache, ob sich die chinesischen Lernenden in China oder im Ausland aufhalten. Zur Sprachbarriere und möglicherweise unterschiedlichen akademischen Ansätzen komme noch der meist große kulturelle Unterschied mit den damit verbundenen Schwierigkeiten:

However, Asian students who are studying in a second language (L2) frequently face a triple challenge: not only must they master the content and concepts of their discipline, and do so through the medium of a language which they may not fully command, but frequently they must do this within an educational and cultural context quite different from their own (Kirby et al. 1996: 141).

Auf der einen Seite werden der Fleiß und das zielstrebige leistungsorientierte Lernen chinesischer Studierender gelobt (Gao 2003: 41), auf der anderen Seite werden unterschiedliche Vorstellungen vom Lernen kritisiert. Sprachkenntnisse seien essentiell für das akademische „Überleben“ und den Erfolg, jedoch zeigten sich besonders in der ersten Zeit gravierende Defizite sowohl in der akademischen wie in der sprachlichen Studierfähigkeit (ebd.). Kennzeichnend für das Verhalten der Studierenden in nicht-chinesischer Lernumgebung sei, dass sich viele Studierende nicht oder nur wenig in anderskulturelle Studierendengruppen integrieren und häufig in der eigenen (Sprach)gruppe verblieben, was ihren Spracherwerb nicht fördere. Ebenso fiele es den meisten chinesischen Studierenden außerordentlich schwer, ein Studium selbständig und eigenverantwortlich zu gestalten (Zeilinger 2006: 1): selbständiges Erarbeiten von Unterrichtsaspekten und Lernerautonomie müssten zunächst einmal gelehrt und trainiert werden.

Hinter vielen Aussagen zur Effektivität heutzutage praktizierter kulturspezifischer Lernweisen steht die Annahme, dass Lerntraditionen eine entscheidende Rolle im Lernverhalten bestimmter Lernergruppen spielen. Einigkeit besteht dar- 
über, dass chinesische Studierende z. T. andere Lehr- und Lernmethoden bevorzugen als etwa ihre europäischen Kommilitonen, ohne dass mit dieser Aussage etwas über die Effizienz ausgesagt würde (Mitschian 1992; Zeilinger 2006).

Westliche Lernende halten Sprachbegabung und Talent für einen nicht zu unterschätzenden Teil ihrer Sprachlerneignung bzw. des möglichen schnellen Erfolgs. Für chinesische Lernende spielt dies nur eine untergeordnete Rolle, harte und fleiBige Arbeit ist für sie durchaus ein Motor zum gewünschten akademischen bzw. Sprachlernerfolg (Biggs \& Watkins 1996: 277). Die Vorstellung vom Lernen als lebenslanger Prozess und davon, dass Fleiß und harte Arbeit genau so wichtig wie Begabung und Talent sind, stellt eine der wichtigsten Motivationskräfte der meisten chinesischen Studierenden dar. Eine Fokussierung auf ein bestimmtes Ziel das Bestehen einer Prüfung oder ein nahendes Auslandsstudium - und Disziplin sind demnach sehr wichtig für ein Studium und setzen strenges Selbststudium und Vorbereitung auf den Unterricht für einen Erfolg voraus (Watkins \& Biggs 2001; Cortazzi \& Jin 2001). Mitschian $(1997,1999)$ führt die Spezifika asiatischer und besonders chinesischer Lernender vor allem auf lernkontextuelle Faktoren wie schulische Sozialisation und die Studienorganisation zurück. Gegen die viel verbreitete Vorstellung, diese gingen auf konfuzianische Lehr- und Lerntraditionen zurück, wendet sich Mitschian, indem er nachweist, dass konfuzianische Schriften vielmehr die Ausbildung zum selbständigen Lernen propagieren und die Anleitung, nicht die Erklärung die häufigste Führungsmethode sei, so dass die Studierenden motiviert würden, selbst den Sinn des Lernens zu erkennen (Mitschian 1997: 394).

Auch Zeilinger (2006: 1) konstatiert Charakteristika wie Gruppenorientierung, Passivität, Schweigsamkeit und Kritiklosigkeit als typisch für chinesische Lernende. Diese Eigenschaften führten auf Seiten von Lehrenden und Beratenden zu Ratlosigkeit gegenüber den Problemen chinesischer Studierender. Haben es Lehrende in Deutschland mit Studierenden aus China zu tun, ergeben sich in verschiedenster Richtung demnach immer wieder zahlreiche Probleme, die sich auf den gesamten Deutschunterricht bzw. das Studium in Deutschland beziehen.

Einige Aussagen deutscher Lehrender aus der Praxis, mögen die zahlreichen Beobachtungen über das Verhalten chinesischer Studierender im deutschen universitären System - und dies bezieht sich sowohl auf den Deutschunterricht als auch auf ihre allgemeine Studierfähigkeit - illustrieren (vgl. Krull 2004):

Chinesische Studierende

- zeigen einen Mangel an Aktivität, der sich in allgemeiner Diskussionsscheu äuBert.

- geben kaum ihre persönliche Meinung preis.

- hinterfragen selten Nichtverstandenes.

- verhalten sich zu einem großen Teil rezeptiv.

- zeigen eigene Aktivitäten nur zögernd.

- „vergraben“ sich in der Textrezeption am liebsten in ihr Wörterbuch. 
- zeigen ein aufmerksam freundlich lächelndes Schweigen.

- zeigen Stärken im Memorieren, im Behalten und im fleißigen Sammeln von Belegen.

- schreiben alles mit.

- greifen als Gruppe sofort soufflierend ein, sobald durch das Ausbleiben einer Antwort ein „Stocken“ des Unterrichtsflusses entsteht oder wenn eine individuelle Meinung oder Leistung gefragt ist.

- haben Schwierigkeiten, Zusammenhänge zu erkennen und quer zu denken.

- sprechen sofort alles nach.

- verstehen Aufforderungen/Aufgabenstellungen nicht

Alle hier formulierten Äußerungen weisen - lässt man etwaige Stereotypisierungen außen vor - darauf hin, dass deutsche und chinesische lernkontextuelle Faktoren sowie Lehr- und Lerntraditionen offenbar unterschiedlich sind bzw. wahrgenommen werden. Im Spannungsfeld des Fremdsprachenunterrichts als Faktorenkomplex aus soziopolitischen Faktoren, Lehr- und Lernumgebungsfaktoren, wissenschaftlichen Faktoren, personenbezogenen Faktoren (Lehrende und Lernende) sowie dem Unterricht bzw. dem Curriculum an sich ergeben sich für die Situation chinesischer Deutschlernender zahlreiche Schwierigkeiten (vgl. Edmondson 1984: 57).

Chinesische Lernende haben ein anderes Verhältnis zu ihren Lehrern als deutsche Studierende und auch das Verhalten im Unterricht erscheint westlichen Lehrern ungewohnt. Mitschian (1992: 4) (vgl. auch Cortazzi \& Jin 2001) sieht schon allein in den unterschiedlichen Ausprägungen des Wortes „Ausbildung / Erziehung“ im Chinesischen einen deutlichen Unterschied:

Jiaoyu, das chinesische Wort für Ausbildung/Erziehung, setzt sich aus den Wörtern Jiao und $Y u$ zusammen, wobei Jiao eine Verkürzung von Jiaoshu (Lehren/Unterrichten (wörtlich, die Bücher lehren) darstellt, während der zweite Teil von Yuren (Menschen erziehen, züchten, aufziehen) herrührt. Jiaoyu bedeutet demnach wörtlich: Buchwissen vermitteln und dadurch Menschen erziehen.

$$
\begin{array}{cc}
\text { Jiaoyu } & \text { Ausbildung/Erziehung } \\
\text { Jiaoshu }+ \text { Yuren } & \text { Buchwissen vermitteln }+ \text { Menschen erziehen }
\end{array}
$$

Diese Vorstellung impliziert neben fachlichen Qualifikationen auch einen moralischen Anspruch an den Lehrenden, der viel mehr „erzieherisch“ als ,vermittelnd“ wirken soll. Schon diese kurze Sequenz zum Schlüsselbegriff der Bildung allgemein mag erklären, dass chinesische Sprachlernende und deutsche Sprachlehrende häufig unterschiedliche Vorstellung von der Lehrer-Schüler-Beziehung haben.

Kleppin beschreibt ihre Rolle als Lektorin in China wie folgt: 
Man wird [...] als ausländischer Lehrer, der bisher nur mit meist chancenlosen europäischen Studierenden zu tun hatte, mit Erstaunen und Begeisterung einer Gruppe von hochmotivierten Lernern gegenüberstehen, die alles aufzusaugen und $\mathrm{zu}$ verarbeiten scheinen, was aus dem Mund oder der Hand des Lehrers kommt. Auf der anderen Seite wollen oder können chinesische Studierende meist noch keine eigenständigen Entscheidungen treffen, so dass der Lehrer seinen Unterricht kaum geäußerten Kommunikationsbedürfnissen anpassen kann (1987: 253).

Die Aussage von Kleppin - die sich durchaus mit den Aussagen anderer Lektorinnen und Lektoren deckt, die ich selbst in China erlebt habe - zeigt eine Verunsicherung im Umgang mit chinesischen Studierenden, die sich eben nicht nur auf die Persönlichkeit bezieht, sondern auch auf grundsätzliche Unterschiede in Zielen, Methoden und im Verhalten der Lehrenden den Lernenden gegenüber.

\section{Konzeptualisierungen der Lehrerpersönlichkeit im chinesischen Umfeld}

Asiatische Lerner haben eine sehr konkrete Vorstellung von der Persönlichkeit eines Lehrers und vom Lehren an sich. Aspekte wie Autorität und Respekt - den Lehrer als Vorbild für das Lernen und für Verhaltensstandards betrachtend - prägen den Schul- und Universitätsalltag chinesischer Bildungseinrichtungen. Hinter dieser Vorstellung verbergen sich intellektuelle und moralische Ansprüche an die Lehrenden, die die meisten deutschen Lehrenden wohl nicht einmal an sich selbst stellen. Die lehrerseitige Dominanz - zur Schau gestellt durch nur dem Lehrer vorbehaltenes „Herrschaftswissen“ (Hess 1992b: 705), das z. T. wörtlich wiedergegeben werden muss - bekräftigt die soziale Rolle des Lehrers als intellektuelle Autorität.

Watkins \& Biggs (2001: 119) beschreiben in diesem Zusammenhang einen Hongkonger Mittelschul-Lehrer, der den idealen Lehrer als hingebungsvolle, hart arbeitende, gut gelaunte Persönlichkeit darstellt, die ihren Beruf liebt und moralisch gefestigt und verantwortungsbewusst bis zur Selbstaufopferung ist. Der Lehrer sei wie eine Kerze, die sich voller Selbstaufopferung verzehre. Er verstehe sein Handwerk und sei ein Fachmann, der sich darüber hinaus guter Gesundheit erfreue.

Hess (1992) liefert in seiner starken Kritik an der „sinisierten Fassung“ (Hess 1992b: 693) des Medienpakets „Alles Gute“, das sich in seiner chinesischen Bearbeitung sehr deutlich vom Original unterscheidet, eine detaillierte Beschreibung der unterschiedlichen Vorstellungen, wie Lernprozesse in Deutschland und China verstanden werden. Während die deutsche Fassung auf die Entwicklung eines „Sehverstehens“ gerichtet ist, ,also der Nutzung visueller ,Daten’ und der Fähigkeit, diese zusammen mit vorhandenem Vorwissen hypothesenartig zur Dekodierung der eigentlichen sprachlichen Information zu nutzen“ (Hess 1992b: 697), 
wird die chinesische Fassung durch systematische Grammatik- und Wortschatzerläuterungen (auf Chinesisch) durch einen zusätzlichen Moderator ergänzt; es werde nicht zum „Sehverstehen“ animiert, sondern „von oben herab gelehrt“ (ebd.: 699):

Ein Text, und dies ist die implizite Hypothese über den stattfindenden Lernprozess, ist erst dann ,verstanden', wenn - in eindimensionaler, linearer Reihung - die Summe der in ihm enthaltenen Einzelwörter bekannt ist, d. h. übersetzt werden kann. Die Reduzierung des Lernens zu einer bloßen organisatorischen Unterrichtshülle wird aber noch weiter getrieben. Das Verständnis nämlich wird dabei nicht von den Lernern (Zuschauern) selbst erarbeitet; es wird vielmehr autoritativ durch Übersetzung vorgegeben, durch den Lehrer... (ebd.)

Dass Lehrerinnen und Lehrer eine phonetische Modellrolle innehaben und hierin auch Multiplikatorenfunktion haben (vgl. Dretzke 2006: 132) dürfte hinlänglich bekannt sein, dass asiatische Lernende von ihren Lehrenden aber eben auch eine Modellfunktion hinsichtlich des Verhaltens erwarten, ist so zumindest in europäischen Zusammenhängen häufig nicht klar. Das Konzept der allwissenden - chinesischen - Lehrerpersönlichkeit korrespondiert mit einer hohen Sensibilisierung der Studierenden für die Persönlichkeit des Lehrenden, seine Unterrichtsvorbereitung, seinen Status und sein “Gesicht” (Watkins \& Biggs 2001; zum Konzept „Gesicht“ siehe Weidemann 2004). Die Sorge „Gesicht zu verlieren“, beispielsweise durch das öffentliche Eingeständnis von Nicht-Wissen, führt - gerade im mündlichen Bereich - dazu, dass Fehler von Sprachlernern durch den Lehrer eher ignoriert werden, wenn diese sich unsicher sind bzw. ihren eigenen Sprachkenntnissen nicht trauen. Diese Versagensängste chinesischer Deutschlehrer gelten im besonderen Maße für das Gebiet der Ausspracheschulung, weil es sich hier nicht nur um einen wichtigen Teil des Deutschunterrichts handelt, sondern weil es auch ein sehr sensibler Bereich ist, da eine Kritik an der Aussprache häufig als persönliche Kritik aufgefasst wird, stärker als das etwas bei grammatischen Korrekturen der Fall ist. Für Lehrende und Lernende stellt beispielsweise die Forderung lauter, langsamer oder schneller zu sprechen, den Mund weiter zu öffnen oder zu schließen oder physiologisch ungewohnte Bewegungen zu machen - im wahrsten Sinne des Wortes und im übertragenen Sinne - eine Kritik am „Gesicht“ dar und wird vielfach als Angriff auf die Gesamtpersönlichkeit wahrgenommen.

Haben Lehrer dazu noch selbst eine fehlerhafte Aussprache und sind sich dessen bewusst, wird vermutlich noch weniger korrigiert. Darüber hinaus sind Lehrer häufig wenig geschult Fehler zu hören oder gar zu analysieren, d.h. sie können vielleicht noch die Fehler heraushören, wissen aber nicht, wie sie darauf reagieren sollen und/oder es fehlen ihnen die Mittel und das Wissen Wege zur Korrektur aufzuzeigen. Viele chinesische Deutschlehrende haben daher auch große Angst vor der Fehlerkorrektur und müssen auf diesem Gebiet aus- und fortgebildet werden. Mit entsprechender Sensibilität und geeigneten Unterrichtskonzepten lässt sich dem „Gesichtsverlust“ auf Seiten der Lehrenden und Lernenden sehr wohl entgegenwirken, beispielsweise durch individuelle Förderung oder Möglichkeiten der Selbstkorrektur und des autonomen Lernens. 


\section{Lernziele chinesischer Studierender}

Eine gute Aussprache als eines von mehreren anzustrebenden Zielen im Fremdsprachenunterricht spielt für die meisten chinesischen Studierenden keine Rolle. Sie lernen häufig mit viel pragmatischeren Interessen als ihre westlichen Kommilitonen. Intrinsische Motive, wie etwa das Interesse an deutscher Kultur oder der Sprache selbst bzw. deren Aussprache, spielen dabei eher eine untergeordnete Rolle. Für die meisten chinesischen Deutschlernenden ist das Bestehen einer bestimmten Prüfung des Bildungssystems (z.B. Aufnahmeprüfungen für weiterführende Studiengänge, Stipendienprogramme oder Sprachprüfungen, die mit einem eventuellen Deutschlandstudium zusammenhängen) die höchste Motivation für das Studium. Dies führt dazu, dass die Studierenden hochgradig prüfungsorientiert lernen (Watkins \& Biggs 2001, Zeilinger 2006).

Auch die Curricula sind demnach an Prüfungen, nicht an Zielen zum Erreichen bestimmter Fertigkeiten orientiert. Als Prüfungsformen dienen dann meist Klausuren oder schriftliche Arbeiten, so dass mündliche Kommunikationsfähigkeit keine oder kaum eine Rolle spielt, d. h. in den üblichen Prüfungsformen nicht abgeprüft wird. Sind mündliche Prüfungen Bestandteil von Prüfungen oder Tests, ist üblicherweise darüber hinaus die Ausspracheleistung kein Bewertungskriterium, d. h. es besteht aus dieser Sicht heraus kein Bedarf nach guten Ausspracheleistungen zumindest nicht im Heimatland.

$\mathrm{Zu}$ individuellen Zielen und Motiven für die Ziele der Studierenden kommt häufig noch der Erfolgsdruck hinzu, der durch andere Personen aufgebaut wird: Familie, die soziale Gruppe oder „die Gesellschaft als Ganzes“ (Biggs \& Watkins 1996: 274) erwarten Leistung für die in den einzelnen Studierenden gesetzten Ambitionen. Kollektivistische Ansichten wie der Drang nach Stabilität und Ordnung als Ausdruck der Loyalität gegenüber der Familie sind dabei entscheidend. Zumindest in chinesischer Lernumgebung spielen auch generelle kollektivistische Orientierungen eine weitere große Rolle im Leistungswettkampf. Auf der einen Seite steht die streng hierarchisch orientierte Lehrer-Schüler-Beziehung, auf der anderen eine nicht nur auf den Unterricht beschränkte Gruppendynamik bzw. ein Gruppendruck, der einzelne verpflichtet gruppengemäß zu handeln.

Haben die Studierenden ihr vordergründigstes Ziel - die Aufnahme eines Studiums an einer deutschen Universität - erreicht, nimmt die prüfungsorientierte Motivation häufig ab, da nun zunächst der für viele Studierende schwierig zu bewältigende „,normale“ Studienalltag in den Vordergrund tritt und Prüfungen bzw. Lernkontrollen in weitere Ferne rücken. Hier kommt es nun wieder zunächst darauf an, verstanden zu werden; eine gute Aussprache wird für scheinbar nebensächlich gehalten. Nach einem kurzzeitigen starken Lernfortschritt auf Grund der neuen - nun deutschen - Lernumgebung fossilisieren die Sprach- und Sprechleistungen dann häufig (vgl. Mehlhorn 2006: 228). 
Dabei könnten unterschiedlichste Formen von Sprachlernberatung oder auch Aussprachelernberatung hilfreich sein, neue Motive herauszubilden und neue Ziele zu setzen (Zeilinger 2006, Mehlhorn 2006).

Bisherige Desiderata der sprechwissenschaftlichen und Deutsch-als-Fremdsprache-Forschung wie Untersuchungen zur Verständlichkeit von Äußerungen bzw. zur Akzeptanz beim Hörer sowie zur emotionalen Wirkung von Gesprochenem wie sie von Hirschfeld (2001b: 89) angemahnt werden, könnten hier Aufklärung bringen. Im Rahmen von Einzelfallstudien zu meiner Dissertation wurden Polaritätenprofile wie das abgebildete (vgl. Abb. 1) erstellt. Einer Hörergruppe wurden dazu die Aufnahmen von drei vorgelesenen Textes mit der Aufgabe vorgespielt, die Wirkung des ausländischen „Akzents“ mit Hilfe von vorgegebenen gegensätzlichen Adjektivpaaren zu beschreiben. Obwohl die Muttersprachen der Deutschlernenden nicht bekannt waren, wurden sie von fast allen (20 von 22) Hörern erkannt. Die chinesische Sprecherin wurde im Vergleich zu anderen Deutschlernenden tendenziell negativ bewertet. Im Beispiel ist zwar zu sehen, dass die russische Muttersprachlerin noch negativer bewertet wurde (erkennbar am Minus hinter dem durchschnittlichen Wert), der portugiesische Muttersprachler wurde hingegen bis auf einen Wert durchgehend positiver bewertet. Würde man beispielsweise dieser chinesischen (oder im konkreten Fall auch der russischen) Studierenden vor Augen führen, wie der chinesische „Akzent“ durch deutsche Hörer bewertet wurde, nämlich überdurchschnittlich negativ bzw. ,anstrengend/angestrengt", wäre sicher der Wunsch nach einer Verbesserung dieses vermeintlich schlechten Images chinesischer Studierender ein weiterer motivationsfördernder Aspekt.

\begin{tabular}{|c|c|c|c|c|c|c|c|c|c|c|}
\hline & 1 & 2 & 3 & 4 & 5 & 6 & 7 & & russisch & $\begin{array}{l}\text { portu- } \\
\text { giesisch }\end{array}$ \\
\hline angenehm & & & & 4,05 & & & & unangenehm & $4,5-$ & $2,8+$ \\
\hline akzeptabel & & & 3,18 & & & & & inakzeptabel & $3,7-$ & $2,3+$ \\
\hline interessant & & & 3,86 & & & & & uninteressant & $3,9-$ & $2,8+$ \\
\hline anregend & & & & 4,5 & & & & ermüdend & $4,6-$ & $3+$ \\
\hline anziehend & & & & 4,45 & & & & abstoßend & $4,5-$ & $3,1+$ \\
\hline vertraut & & & & 4,82 & & & & ungewohnt & $4,6+$ & $2,8+$ \\
\hline sympathisch & & & 3,73 & & & & & unsympathisch & $3,8-$ & $2,5+$ \\
\hline ausdrucksvoll & & & & 4,05 & & & & ausdruckslos & $4,6-$ & $3,2+$ \\
\hline bewegt & & & 3,73 & & & & & unbewegt & $4,9-$ & $3,3+$ \\
\hline engagiert & & 2,73 & & & & & & unengagiert & $4,5-$ & $2,9-$ \\
\hline farbig & & & 3,90 & & & & & farblos & $4,9-$ & $3,2+$ \\
\hline warm & & & & 4,18 & & & & kalt & $4,6-$ & $3,3+$ \\
\hline entspannt & & & & & & 6,18 & & angestrengt & $5,6+$ & $3,9+$ \\
\hline
\end{tabular}




\begin{tabular}{|c|l|l|l|l|l|l|l|c|c|c|}
\hline erholsam & & & & & 5,43 & & & anstrengend & $5,8-$ & $3,6+$ \\
\hline weich & & & & & 5,05 & & & hart & $4,9+$ & $3,3+$ \\
\hline niedlich & & & 3,86 & & & & & derb & $4,2-$ & $2,9+$ \\
\hline
\end{tabular}

Abb. 1: Polaritätenprofil einer Sprecherin, Muttersprache Chinesisch, durchschnittliche Bewertung (22 Bewertungen durch Teilnehmer eines Seminars zur Kontrastiven Phonetik / Fehleranalyse Herder-Institut Leipzig) und zum Vergleich Bewertungen für eine russische Muttersprachlerin und einen portugiesischen Muttersprachler, die beide den gleichen Text lesen wie die chinesische Muttersprachlerin.

\section{$5 \quad$ Methoden des Lehrens und Lernens}

Methoden des kommunikativen Ansatzes werden von Studierenden, die bisher konventionellere Methoden (v. a. Grammatik-Übersetzungs-Methode) gewöhnt waren, häufig zu Beginn abgelehnt. Eine Thematisierung der Lehr- und Lernmethoden führt aber auch zu einem Umdenken und einer guten Annahme von neueren Ansätzen (Dai 2001; Zeilinger 2006). Einige der ,konventionellen“ Techniken, die hier von Dai angesprochen werden, wie etwa das laute Lesen von Texten, könnten durchaus für die Ausspracheausbildung genutzt werden. Sie müssen allerdings durch Kontrollmechanismen (beispielsweise regelmäßige Aufnahmen, die dann von Lehrenden oder auch den Lernenden selbst kontrolliert werden) ergänzt werden, damit das laute Lesen nicht nur für sich selbst steht, sondern kontextuell in den Unterricht bzw. den gesamten Lern- und Erwerbsprozess eingebunden wird.

Ausgangspunkt der meisten chinesischen Lehrmaterialien und -methoden sind Texte (Cortazzi \& Jin 2001), wobei die Lehrenden ihr textbasiertes, z. T. sehr zeitaufwändiges Lehren aufmerksam und detailliert planen. Mündliche Kommunikationsfähigkeit spielt dagegen in den meisten Kursen nur eine sehr untergeordnete Rolle, weswegen schriftliches Lernen (Gao 2003: 54) mit viel Zeitaufwand für Vorund Nachbereitung eine starke Priorität gegenüber mündlichem Lernen hat. So wirkt sich dies im Ergebnis auch auf die phonetischen Leistungen der Lernenden aus. Der mündliche akademische Diskurs im Studium wird wie das Einüben mündlicher Sprachfertigkeiten in der chinesischen Ausbildung bisher nicht oder nicht ausreichend gepflegt, weil Vorlesungen und Seminare, aber auch Sprachkurse mit starken lehrerseitigen Redeanteilen die üblichen Lernformen sind.

Für chinesische Deutschlernende ist Deutsch in den meisten Fällen die zweite Fremdsprache nach Englisch. Dennoch fehlt es ihnen häufig an Sprachbewusstheit und Sprachbewusstsein sowohl über ihre erste Fremdsprache als auch über die Muttersprache Chinesisch. Rückschlüsse, die das Lernen der zweiten Fremdsprache erleichtern könnten, werden häufig nicht wahrgenommen oder nicht verstanden. Vergleiche zur chinesischen Sprache, die das Lernen erleichtern könnten oder die einfach nur zur Sichtbarmachung von Phänomenen der jeweiligen Sprachen 
dienen könnten, sind nicht Teil des Unterrichts, wohl auch nicht des Chinesischunterrichts an chinesischen Mittelschulen bzw. Gymnasien.

Im Zusammenhang mit der Analyse unterschiedlicher Lernkulturen bzw. der Missverständnisse zwischen chinesischen Studierenden und britischen Lehrenden in Kursen zum studienbegleitenden Englischunterricht sehen Cortazzi \& Jin (2001) eine Reihe von Dilemmas. Üblicherweise würden Studierende aus asiatischen Ländern („Confucian-heritage cultures“ ebd.) unter Lernbedingungen unterrichtet, die gemessen an westlichen Standards suboptimal wären: große Klassen, vorlesungsartige Methoden (vgl. auch DAAD 2005) mit unnachgiebigen normreferentiellen Assessments in strengem Klassenklima. Dennoch erzielten sie gleiche oder bessere Leistungen (besonders in naturwissenschaftlichen Fächern) als vergleichbare westliche Lernende. Asiatische Studierende seien darüber hinaus ,,passive Auswendiglerner" (Cortazzi \& Jin 2001), erzielten aber ein großes Maß an Verständnis. Das Gegensatzpaar der westlichen Betrachtung Auswendiglernen vs. Verstehen besteht allerdings so nicht. Chinesische Studierende nutzen vielmehr eine Art „verstehendes Auswendiglernen“ (Marton et al. 1996) als Lernstrategie. Ausgehend von der allgemeinen Prämisse, dass Auswendiglernen nur beschränkt zu guten Ergebnissen in (Sprach)lernprozessen führt, müssten chinesische Studierende generell also schlechtere Leistungen erbringen als Studierende aus anderen Weltregionen, was empirisch nicht zu bestätigen ist (Marton et al. 1996, Watkins \& Biggs 2001). Gelöst wird dieses scheinbare Paradox durch den Ansatz von Marton et al. (1996: 82), die mit Hilfe ihrer Forschungen mit Lehrkräften aus der VR China und Hongkong herausgefunden haben, dass unter „Auswendiglernen“ im westlichen Forschungskontext „mechanisches Auswendiglernen“ verstanden werde, das nicht zwangsläufig mit Verstehen einher geht. Der gesamte Prozess des Auswendiglernens - so wie es die chinesischen Lehrkräfte sehen - führe demgegenüber zur Vertiefung und Entwicklung des Verstehens:

In Western countries memorization and rote learning are generally equated and it is commonly believed that they do not lead to understanding. A new way of seeing the relationship between memorization and understanding as being intertwined was identified in this study. In addition, a distinction was found 'within' memorization, rather than 'between' memorization and understanding. These results enable us to see the traditional Asian practice of repetition or memorization can have different purposes. On the one hand, repetition can be associated with mechanical rote learning; on the other hand, memorization can be used to deepen and develop understanding. If memorization is understood in this latter way, the paradox of the Asian learner is solved (Marton et. al 1996: 82).

Nutzt man also im Ausspracheunterricht diesen Aspekt aus, können Methoden, die im westlichen Kontext eher auf Ablehnung treffen, durchaus zu guten Ergebnissen führen. Beispielsweise könnten formelhafte Texte, die bestimmte prosodische Formen beinhalten - einmal auswendig gelernt - dazu dienen, prosodische Merkmale zu schulen und ihnen modellhaften Charakter für andere Inhalte zu geben. 
In der Literatur finden sich häufig Hinweise auf die „Passivität“ chinesischer Studierender (Kleppin 1987; Mok et al. 2001; Zeilinger 2006). Sie wird häufig mit der Angst vor Fehlern, die einen Gesichtsverlust nach sich ziehen würden, oder mit dem Wunsch, innerhalb der Gruppe nicht aufzufallen und somit die Gruppenharmonie nicht zu stören, begründet. Fragen stellen würde als Kritik am Lehrenden empfunden und das Beantworten von Fragen ohne direkt aufgefordert worden zu sein würde als Ausbrechen aus dem Klassenverband betrachtet. Bürner-Kotzam \& Jensen (1988: 69) haben eine ,grundsätzliche Zurückhaltung der Studentinnen mit ihrem aufmerksamen freundlich lächelnden Schweigen" beobachtet, die Fragen zwar leise, aber meist korrekt beantworten und sich ansonsten passiv verhielten. Diese ,als ,fein' empfundene Zurückhaltung entspreche immer noch dem Frauenideal“, so begründeten die Studentinnen ihr Verhalten (ebd.). Im Zusammenhang mit möglicherweise kulturell bedingter Passivität chinesischer Studierender werden mögliche Ursachen beschrieben:

Wäre die Passivität kulturell bedingt, müsste der Lehrer diese akzeptieren und seine Lehrmethode danach ausrichten. Sei jedoch davon auszugehen, dass die Passivität ein Resultat der schulischen Sozialisation sei, dann könne der Lehrende den Versuch unternehmen, dieses Lernverhalten zu ändern (Zeilinger 2006: 7; vgl. Mitschian 1999).

Eigene Erfahrungen im Lehrbetrieb und beispielsweise Unterrichtskonzepte für Kurse des Goethe-Instituts in der VR China bestätigen die Richtigkeit der oben beschriebenen Hypothese, dass nämlich das chinesische - vermeintlich kulturell bedingte passive Lernverhalten - doch eher auf die schulischen Erfahrungen und Sozialisation bzw. akademische Zwänge zurückzuführen ist, als auf oftmals damit in Zusammenhang gebrachte Traditionen wie die Vorbereitung auf Kaiserliche Beamtenprüfungen oder Ähnliches.

Ein großer Teil des sehr lehrerzentrierten Unterrichts erfolgt im Frontalunterricht (Cortazzi \& Jin 2001; Zeilinger 2006). Dies gilt auch für Sprachkurse, die meist in Gruppen von ca. 20 Personen, häufig auch mit mehr Studierenden abgehalten werden. Ist Deutsch im Rahmen des Studiums die zweite Fremdsprache neben einem anderen Sprach- oder Fachstudium, hat der Sprachunterricht z. T. sogar Vorlesungscharakter mit entsprechender Gruppengröße von z. T. 70 bis 120 Studierenden, wovon ich mich selbst als DAAD-Lektorin in China überzeugen konnte.

Im Rahmen meiner Untersuchung habe ich 2003 eine Befragung von 32 deutschen und 33 chinesischen Deutschlehrenden in Beijing durchgeführt, bei der verschiedene Situationen gegeben wurden, die die Lehrenden einschätzen sollten. Eine Situation beschäftigt sich mit der Korrektur, zwei andere Situationen mit Gruppenarbeit. Einige exemplarische Antworten sind in den folgenden Tabellen aufgeführt. 
Sie unterrichten im Grundkurs Aussprache. Einer Ihrer Studenten sagt: „Bitte korrigieren Sie mich jedes Mal, wenn ich einen Fehler mache!“

Antworten der deutschen Lehrenden

- Ihm vermitteln, dass ich ungern seinen Redefluss störe, aber am Ende seiner Wortmeldung auf Fehler hinweisen werde.

- Ich würde vorschlagen, mich zunächst auf die gravierenden Probleme zu konzentrieren und dann schrittweise zu anderen vorzuschreiten.

- Ich mache ihm klar, dass eine Korrektur z.B. beim Vorlesen oder bei Nachsprechübungen immer erfolgt, dass dies jedoch nicht der Fokus des Unterrichts ist, da es sich um eine Gruppe von Studenten handelt, die sämtliche Fertigkeiten erlernen soll. Ich gebe aber Tipps für vertiefende Übungen.

- Darauf kommt es nicht an, Hauptsache ich verstehe dich, höchstens bei grundsätzlichen Fehlern.

- Ich würde diesem Wunsch selbstverständlich nicht entsprechen und erklären warum.

- Ich korrigiere die größten Fehler und mache mir Notizen und korrigiere anschließend. Im Detail ist das schwierig in der Klasse. Ich weise darauf hin, dass es zunächst auf Verständlichkeit ankommt.
Antworten der chinesischen Lehrenden

- Bitte sprechen sie weiter.

- Ich bin sehr froh, dass Sie bereit sind, korrigiert zu werden. Ich werde mich auch bemühen, Ihre Fehler zu besprechen.

- Ich würde ihn aus Mangel an Zeit nicht jedes Mal korrigieren. Typische Fehler vieler Studenten würde ich schon gleich korrigieren.

- Wenn wir phonetische Übungen machen, mache ich das gern. Wenn wir aber über Inhaltliches sprechen, mache ich es erst im Nachhinein.

- Am Anfang verstehe ich das und bemerke es auch, aber im Hauptstudium werde ich das nicht machen.

- Die Aussage ist frech. Ich würde mich ärgern.

- Das ist nicht nötig, denn eine sehr gute Aussprache hat man erst dann, wenn man sich ausreichende Sprachkenntnisse angeeignet hat.

- Wenn ich das machen würde, könnten Sie nie einen Satz zu Ende sprechen. Und manche Fehler machen mehrere Studenten, die werden dann bei den anderen korrigiert.

- Tut mir leid, ich bin leider nicht dein Privatlehrer.

Sie machen im Unterricht viel Gruppenarbeit. Ein Student sagt: „Wir reden zwar viel im Unterricht, aber ich lerne nichts, weil mein Sprachpartner eine schlechtere Aussprache hat als ich."

\begin{tabular}{|c|c|}
\hline Antworten der deutschen Lehrenden & $\begin{array}{l}\text { Antworten der chinesischen } \\
\text { Lehrenden }\end{array}$ \\
\hline $\begin{array}{l}\text { - In Deutschland sprechen auch nicht alle astreines } \\
\text { Hochdeutsch. Es ist eine gute Übung, um ver- } \\
\text { schiedene Aussprachen, auch nicht ganz korrekte } \\
\text { verstehen zu lernen. } \\
\text { - Er soll die Fehler des anderen analysieren. Ich } \\
\text { ändere regelmäßig Gruppenzusammensetzungen } \\
\text { und weise darauf hin, dass jeder andere Kompe- } \\
\text { tenzen mitbringt. }\end{array}$ & $\begin{array}{l}\text { - Übung macht den Meister. } \\
\text { Fortschritte können nicht über } \\
\text { Nacht, sondern nur allmählich } \\
\text { erzielt werden. Man soll in } \\
\text { der Gruppenarbeit einander } \\
\text { helfen und gemeinsam Fort- } \\
\text { schritte machen. }\end{array}$ \\
\hline
\end{tabular}


- Ich mache ihm klar, dass dies auch eine Übung/ ein Training in Hörverstehen sein könnte. Der zentrale Aspekt der Gruppenarbeit ist das kommunikative Handeln und das erfolgt schon beim versichernden Nachfragen.

- Ich kenne dieses Problem nicht, aber wenn es das gibt, würde ich Studien dazu suchen und sie dem Studenten vorstellen.

- Das würde ich ihm ausreden.

- Es geht auch darum, die eigene Sprechfertigkeit zu trainieren und von der guten Aussprache kann der schwächere Partner nur profitieren. Die eigene (gute) Aussprache verschlechtert sich dadurch nicht zwangsläufig.
- Wenn Sie anderen helfen, eine falsche Aussprache zu korrigieren oder eine schlechte Aussprache zu verbessern, lernen Sie dabei auch viel.

- Ich würde überlegen, wie ich meinen Unterricht verbessern kann.

- Jeder hat seinen eigenen Akzent. Sie sollen sich bemühen, ihn zu verstehen und ggf. seine Aussprache korrigieren.

Auffällig im Vergleich von deutschen und chinesischen Deutschlehrenden ist, dass deutsche Lehrkräfte viel mehr mit den Studenten ÜBER den Unterricht sprechen oder den Studenten bewusst erklären, warum sie an welcher Stelle etwas Bestimmtes tun. Das führt letztlich dazu, dass Methoden erklärt werden und Vorurteile gegenüber bestimmten Übungstypen bzw. Sozialformen ausdiskutiert werden. Missverständnisse, die entstehen, weil beispielsweise bei bestimmten Übungstypen die Aussprache nicht korrigiert wird, an anderer Stelle aber schon, können so aufgeklärt werden. Voraussetzung ist allerdings, den chinesischen Studierenden diese Form der aktiven Mitarbeit nahe zu bringen, weil letztlich im Selbstverständnis von Schülern und Lehrenden in China die Lehrerpersönlichkeit immer noch "Respektsperson" ist, die unfehlbar ist und deren Aussagen und Forderungen nicht hinterfragt werden. Die Thematisierung bestimmter Verhaltensmuster der Lernenden im Unterricht würde etwaige Missverständnisse klären bzw. vermeiden helfen, genau so wie die Thematisierung der Methoden unerlässlich ist. Die Aktivierung mündlicher Sprechfertigkeit und damit auch die aktive Arbeit an Aussprache könnten unter der Voraussetzung, dass den Lernenden neue Konzepte und Methoden plausibel gemacht werden, von größerem Erfolg gekrönt sein.

\section{Schlussfolgerungen}

Die vorangegangenen Kritikpunkte führen zu dem Schluss, dass in Zukunft ein wesentlicher Aspekt der lernerorientierten methodisch-didaktischen Arbeit und Begleitforschung auf dem Gebiet der Lehreraus- und -fortbildung liegen muss. Im Prinzip wird seit den 1980er Jahren eine zum Teil nicht ausreichende Ausbildung chinesischer Deutschlehrender bemängelt (Kleppin 1987: 252; Hess 1992b: 709), die sich negativ auf die Lernleistungen der Deutschlernenden auswirkt. Dabei seien sowohl im sprachlichen als auch im methodisch-didaktischen Bereich häufig als ungenügend ausgebildet zu bezeichnende Lehrende tätig. Im Wesentlichen werde versucht, Leseverständnis anhand von Übersetzungsübungen zu entwickeln, wobei 
der Unterricht fast generell in chinesischer Sprache erfolgt (Kleppin 1987: 252). Besonders, wenn Deutsch nicht im Rahmen eines Germanistikstudiums oder in Deutschland, sondern als zweite Fremdsprache gelernt wird, trifft dies auch heute noch zu, wobei sich inzwischen die Anzahl der in Deutschland oder durch verschiedene Fortbildungsprogramme in China (z. B. Goethe-Institut) ausgebildeten chinesischen Deutschlehrenden wesentlich erhöht hat. Erst seit kurzem gibt es überhaupt an einigen Universitäten eine Ausbildung für Deutsch-als-Fremdsprache in China. Zuvor rekrutierten die Universitäten ihren Nachwuchs üblicherweise aus den Absolventinnen und Absolventen der Germanistik-Studiengänge, denen eine didaktisch-methodische Ausbildung komplett fehlt.

Im Zusammenhang mit Hinweisen, die darauf abzielen, dass Lehrende dazu tendieren, diejenigen Methoden in ihrem Unterricht einzusetzen, mit denen sie selbst gelernt haben (Mitschian 1992: 4), d. h. im Falle China gerade ein relativ schriftbasiertes Lernen, muss eben genau in der Lehrerausbildung der Schwerpunkt zukünftiger Bestrebungen liegen. Methoden zur Aussprachevermittlung beschränken sich demzufolge häufig noch auf Nachsprechen-Lassen und die theoretische Erklärung von z. B. Lippen- und Zungenstellung und Mundöffnungsgrad. Lehrende, die auf eine gute Aussprache der Studierenden achten, ernten allzu oft besonders in den höheren Semestern - verständnislose Blicke, weil sie überhaupt nicht darauf eingestellt sind korrigiert zu werden, obwohl sie inhaltlich verstanden wurden. Gerade was phonetisch/phonologische Kenntnisse betrifft, bestehen unter chinesischen Lehrenden starke Defizite. Auch z. T. sehr gut ausgebildete Germanisten haben häufig keinerlei theoretische oder praktische Kenntnisse auf dem Gebiet der Phonetik/Phonologie bzw. deren Vermittlung.

Die Forderung nach einer besseren Lehreraus- und -fortbildung beschränkt sich allerdings nicht nur auf die chinesischen Lehrkräfte. Für deutsche bzw. ausländische Lehrende gilt Entsprechendes. Gao (2003), der sich in seinem Artikel mit Lernstrategien chinesischer Studierender bei ihrer Ankunft in Großbritannien beschäftigt, sieht sowohl Nachholbedarf auf Seiten der chinesischen Lehrkräfte, die Studierende auf einen Aufenthalt im Ausland vorbereiten, und fordert sie dazu auf, die Fremdsprachenausbildung mit interkultureller Ausbildung zu vernetzen und das vorwiegend prüfungsorientierte Lernen zugunsten des Einübens authentischer Lern- und Lehrsituationen, Lernstrategientrainings und der Schulung des eigenen Urteilsvermögens zu favorisieren (Gao 2003: 56). Gleichzeitig fordert er von westlichen Lehrkräften, sich aktiver bewusst zu machen, wie groß die Unterschiede zwischen den akademischen Kulturen sind und dementsprechend zu handeln.

\section{Schlussbemerkungen}

Wurden früher in den Publikationen zum Fremdsprachenerwerb Ausspracheprobleme fast ausschließlich auf sprachliche Interferenzen mit der Muttersprache bzw. anderen gelernten Fremdsprachen zurückgeführt, wurde in diesem Artikel deutlich 
gemacht, dass durchaus auch - und nicht nur zu vernachlässigbar kleinen Anteilen - außersprachliche Faktoren beim Aussprachelernen zu beachten sind. Ansatzpunkte, welchen Einfluss die Lehr- und Lernumgebung auf das Lernen der deutschen Aussprache haben kann, wurden hier dargestellt.

Notwendig ist:

- die Verbesserung des Angebots an Sprachlern- und Ausspracheberatung mit dem Wissen über die chinesische Lernkultur;

- die Nutzung auch der konventionellen „chinesischen“ Methoden (auswendig lernen, laut lesen) für die Ausspracheausbildung (Dai 2001);

- die Förderung des mündlichen akademischen Diskurses in der Rezeptions- und Produktionsphase zur Automatisierung phonetisch-phonologischen Wissens und Könnens mit Hilfe des Einübens authentischer Lern- und Lehrsituationen;

- die Verbesserung der Lehreraus- und -fortbildung (d.h. vor allem Schulung des Beurteilungsvermögens der Aussprache von Lernenden (Gao 2003: 56)), vor allem für chinesische Lehrende;

- Schulung des interkulturellen Wissens und Handelns im Kontext unterschiedlicher akademischer Kulturen, vor allem für westliche Lehrende.

Vergegenwärtigt man sich, dass chinesische Studierende trotz oder vielleicht sogar wegen der oben zusammengefassten Faktoren fachlich-inhaltlich gesehen (v. a. in den Naturwissenschaften, vgl. Cortazzi \& Jin 2001) die gleichen oder gar bessere Leistungen als westliche Lernende erreichen, sind die - zumindest für den europäischen Leser - scheinbar negativ konnotierten Merkmale nicht unbedingt negativ zu sehen. Es geht nun darum, die herausgearbeiteten Kenntnisse so zu nutzen, dass auch für die Ausspracheleistungen positive Ergebnisse erzielt werden.

\section{Literatur}

Biggs, John \& Watkins, David (1996): The Chinese Learner in Retrospect. In: Watkins, David \& Biggs, John (Hrsg.): The Chinese Learner: Cultural, Psychological and Contextual influences. Hongkong, Melbourne, 269-285.

Bürner-Kotzam, Renate \& Jensen, Jörn (1988): Der deutsche Faschismus: Ideologie, Literatur, bildende Kunst und Architektur. Projektunterricht und Team-Teaching in der VR China. In: Info DaF 15(1), 65-75.

Celce-Murcia, Marianne \& Brinton, Donna \& Goodwin, Janet (2003): Teaching Pronunciation: A Reference for Teachers of English to Speakers of Other Languages. Cambridge.

Cortazzi, Martin \& Jin, Lixian (2001): Large Classes in China: ,Good' Teachers and Interaction. In: Watkins, David \& Biggs, John (Hrsg.), Teaching the Chinese Learner: Psychological and Pedagogical Perspectives. Hongkong, 115-134. 
DAAD (2005): Länderinformation für internationales Marketing für Bildung und Forschung in Deutschland. China. [www.gate-germany.de/downloads/ nekomako/ dokumentation/laenderinfos/China_Laenderinformation_GATE 2006.pdf; 20.09.2008).

Dai, Yingjie (2001): Moderne Gesellschaft - Traditionelle Lehrgewohnheiten? Überlegungen zur Neustrukturierung des Unterrichts Deutsch als Fremdsprache in China. In: Jahrbuch Deutsch als Fremdsprache 27, 463-473.

Dretzke, Burkhard (2006): Ausspracheschulung im Fremdsprachenunterricht. In: Jung, Udo O. (Hrsg.): Praktische Handreichung für Fremdsprachenlehrer. Frankfurt/Main, 132-140.

Edmondson, Willis \& House, Juliane (2000): Einführung in die Sprachlehrforschung. Tübingen, Basel.

Fluck, Hans-Rüdiger (2004): Zur Geschichte des Unterrichtens von Deutsch als Fachsprache in China (ca. 1870-1980). In: Fachsprache 26(1), 74-83.

Gao, Xuesong (2003): Changes in Chinese Students' Learner Strategy Use after Arrival in the UK: A Qualitative Inquiry. In: Palfreyman, David \& Smith, Richard (Hrsg.): Learner autonomy across Cultures. Language Education Perspectives. New York, 41-58.

Hess, Hans-Werner (1992a): Die Kunst des Drachentötens. Zur Situation von Deutsch als Fremdsprache in der Volksrepublik China. München.

Hess, Hans-Werner (1992b): Max und Melanie in China. Erfahrungen mit einem deutschen Medienpaket. In: Info DaF 19(6), 692-716.

Heublein, Ulrich \& Sommer, Dieter \& Weltz, Birgitta (2004): Studienverlauf im Ausländerstudium. Eine Untersuchung an vier ausgewäblten Hochschulen. DAAD. Bonn [Reihe Dokumente \& Materialien, Ref. Information, Fortbildung, Info-Center].

Hirschfeld, Ursula (2001): Der „fremde Akzent“ in der interkulturellen Kommunikation. In: Bräunlich, Margret \& Neuber, Baldur \& Rues, Beate (Hrsg.): Gesprochene Sprache transdisziplinär. Festschrift zum 65. Geburtstag von Gottfried Meinhold. Frankfurt a. M., 83-91 [Hallesche Schriften zur Sprechwissenschaft und Phonetik; 5].

Hunold, Cordula (2001): Unterrichtsmaterialien zur Phonetik im Unterricht DaF. In: Schlenker, Wolfram (Hrsg.): Intensivkurse Deutsch in China. Beijing, 201-209.

Hunold, Cordula (2005a): Chinesisch. In: Hirschfeld, Ursula \& Kelz, Hans Peter \& Müller, Ursula (Hrsg.): Phonetik International - von Albanisch bis Zulu. [www.phonetikinternational.de; 20.09.2008].

Hunold, Cordula (2005b): Chinesische Phonetik. Konzepte, Analysen und Übungsvorschläge für den ChaF-Unterricht. Bochum.

Hunold, Cordula (ersch. 2008): Untersuchungen zu segmentalen und suprasegmentalen Ausspracheabweichungen chinesischer Deutschlernender. Frankfurt [Hallesche Schriften zur Sprechwissenschaft und Phonetik].

Kirby, John \& Woodhouse, Rosamund \& Ma, Yamin (1996): Studying in a Second Language: the Experience of Chinese students in Canada. In: Watkins, David \& Biggs, John (Hrsg.): The Chinese Learner: Cultural, Psychological and Contextual influences. Hongkong, Melbourne, 141-158.

Kleppin, Karin (1987): Deutsche Lehrer - Chinesische Lerner. Zur Unterrichtssituation an den Hochschulen in China. In: Info DaF 3, 252-260. 
Krull, Christine (2004): Handout zur Tagung „Interkulturelle Kommunikation und Phonetik Blaubeuren“. Unveröff. Handout und Tagungsbeitrag.

Marton, Ference et al. (1996): Memorizing and Understanding: the Keys to the Paradox? In: Watkins, David \& Biggs, John (Hrsg.), The Chinese Learner: Cultural, Psychological and Contextual influences. Hongkong, Melbourne.

Mehlhorn, Grit (2006): Möglichkeiten einer individuellen Aussprache-Lernberatung. In: DaF 43(4), 228-232.

Mitschian, Haymo (1991): Chinesische Lerngewohnheiten. Evaluierungen für den Deutsch-alsFremdspracheunterricht in der Volksrepublik China. Frankfurt a. M.

Mitschian, Haymo (1992): Traditionen im DaF-Unterricht der VR China. Chinatypisches Lernen nach deutscher Methodik? In: Info DaF 19(1), 3-21.

Mitschian, Haymo (1997): „Traditionelles“ Lernen in China. In: Wolff, Armin \& Schleyer, Walter (Hrsg.): Fach- und Sprachunterricht. Gemeinsamkeiten und Unterschiede. Studiengänge Deutsch als Fremdsprache: Von der Theorie zur Praxis. Materialien Deutsch als Fremdsprache 43, 393-407.

Mitschian, Haymo (1999): Passivität asiatischer Lerner - Analyse einer Verhaltensbeschreibung. In: Fluck, Hans-Rüdiger \& Gerbig, Jürgen (Hrsg.): Spracherwerb Deutsch in Ost- und Zentralasien: Lebr- und Lerntraditionen im Wandel. Tübingen, 45-59.

Mok, Ida et al. (2001): Solving the Paradox of the Chinese Teacher? In: Watkins, David \& Biggs, John (Hrsg.), Teaching the Chinese Learner: Psychological and Pedagogical Perspectives. Hongkong, 161-179.

StADaF (2000): Erhebung Deutsch als Fremdsprache. [www.goethe.de/mmo/ priv/60112-STANDARD.pdf; 20.09.2008].

StADaF (2005): Erhebung Deutsch als Fremdsprache. [www.goethe.de/mmo/ priv/1459127-STANDARD.pdf; 20.09.2008].

Statistisches Bundesamt (2006): Fachserie 11, R. 4.1, WS 2005/2006.

Watkins, David \& Biggs, John (Hrsg.) (1996): The Chinese Learner: Cultural, Psychological and Contextual influences. Hongkong, Melbourne.

Watkins, David \& Biggs, John (Hrsg.) (2001): Teaching the Chinese Learner: Psychological and Pedagogical Perspectives. Melbourne.

Weidemann, Doris (2004): Interkulturelles Lernen. Erfabrungen mit dem cbinesischen 'Gesicht': Deutsche in Taiwan. Bielefeld.

Zeilinger, Miriam (2006): Beratung von ostasiatischen Studierenden. In: ZIF 11/2, 21 S. [http://zif.spz.tu-darmstadt.de/jg-11-2/beitrag/Zeilinger1.htm; 20.09.2008]. 


\title{
Kulturelle Deutungsmuster in deutschsprachigen Medienbeiträgen zum EU-Beitritt der Türkei
}

\author{
Isabelle Maringer (Leiprig)
}

\begin{abstract}
Die vorliegende Arbeit beschreibt die Entwicklung einer Methodik, die es ermöglichen soll, kulturelle Deutungsmuster in öffentlichen Diskursen aufzudecken. Anhand eines Fallbeispiels werden die einzelnen sukzessiven Analysephasen vorgestellt. Den theoretischen Ausgangspunkt der Analysen stellt eine Weiterentwicklung des Deutungsmusteransatzes von C. Altmayer dar. Mit Hilfe des neu gewonnenen Instrumentariums soll dem landeskundlichen Unterricht in Deutsch als Fremdsprache $(\mathrm{DaF})$ auf lange Sicht zu einer qualitativen inhaltlichen Aufwertung verholfen werden.
\end{abstract}

\section{Einleitung}

Der vorliegende Text stellt einen Ausschnitt aus meinem Dissertationsprojekt „Kulturelle Deutungsmuster in den deutschsprachigen Mediendebatten um den EU-Beitritt der Türkei“ dar. Das Vorhaben ist in dem noch jungen Bereich der Kulturstudien im Fach $\mathrm{DaF}$ angesiedelt, eine seit langem ausstehende Weiterentwicklung der herkömmlichen Landeskunde.

Diese sieht ihre Aufgabe traditionell in der Vermittlung der deutschsprachigen „Kultur“ im DaF-Unterricht, die sich allerdings meist in allgemein-faktischen oder stereotypen Aussagen über Deutschland bzw. die Deutschen erschöpft, oder sich mangels eigener Inhalte mit willkürlich zusammengestellten Wissensfragmenten aus fachfremden Gebieten, wie z.B. aus der Geschichte, zufrieden geben muss. Grund für dieses Manko ist u.a. das Fehlen an schlüssigen aktuellen Theorien und wissenschaftlich fundierten Konzepten, über die sich dieser Bereich als selbstständige Wissenschaft positionieren könnte.

Daraus ergibt sich die Notwendigkeit einer Transformation der Landeskunde, die sich für die Kulturstudien im Bereich $\mathrm{DaF}$ vor allem über ein neues Verständnis des Begriffs Kultur gestaltet: Kultur wird hier als Gesamtheit von „tradierten Mustern der Welt- und Wirklichkeitsdeutung" (Altmayer 2006: 51), den so genannten kulturellen Deutungsmustern, aufgefasst (1.1).

Anliegen dieses Ansatzes ist es, über die Vermittlung dieser Deutungsmuster bei den Fremdsprachenlernern einen Aufbau kulturbezogener Kompetenzen zu erreichen, der ihnen die Partizipation am deutschsprachigen Alltagswissen und -leben erleichtert. Aufgabe der Kulturwissenschaften ist also die Beschäftigung mit 
diesen kulturellen Deutungsmustern im Sinne ihrer Sichtbarmachung und Rekonstruktion innerhalb von Texten.

$\mathrm{Da}$ für den beschriebenen Bereich kein vollständig entwickeltes methodisches Instrumentarium existiert, das eine Annäherung und Rekonstruktion von Deutungsmustern in Texten ermöglicht, bleibt das Erarbeiten eines wissenschaftlich fundierten Entwurfes ein wichtiges Desiderat der beschriebenen Forschungsausrichtung.

Dementsprechend sieht die vorliegende Arbeit einen ihrer Aufgabenschwerpunkte darin, die beschriebene konzeptionelle Lücke auf methodischem Gebiet zu schließen. Um eben dies leisten zu können, hat sich eine Weiterentwicklung des Deutungsmuster-Ansatzes als notwendig erwiesen; mit Hilfe einer eigenständigen begrifflichen Differenzierung werden aus dem so präzisierten Konzept klare Prämissen entwickelt, die zur Ausarbeitung der erforderlichen Methodik dienen.

Konkret konzentriert sich das hier verfolgte Erkenntnisinteresse auf das Fallbeispiel der kulturellen Deutungsmuster, die sich in Medienbeiträgen des deutschsprachigen Diskurses zum Thema EU-Beitritt der Türkei aufspüren lassen. Besonders zwei Leitfragen stehen dabei im Vordergrund: Welche Deutungsmuster haben sich innerhalb dieses Diskurses etabliert und welche Funktion üben sie aus?

Ausgewählt wurde eben dieser Diskursausschnitt, da die Vehemenz und Emotionalität der medial geführten Debatten vermuten lassen, dass es sich nicht nur um politisch-ökonomische Streitpunkte handelt, sondern Zugehörigkeitszuweisungen und Identitätskonstruktionen der deutschsprachigen Kommunikationsgemeinschaft eine wichtige Rolle spielen. Dies verspricht ein ergiebiges Untersuchungsfeld für Deutungsmuster zu liefern, innerhalb dessen diese fortwährend zur Bestätigung oder Widerlegung herangezogen werden.

Ziel ist die Annäherung an Deutungsmuster, die sich auf das Verständnis von Europa beziehen und sich dazu auch exklusiver und inklusiver Ab- bzw. Eingrenzungsstrategien bedienen. Dazu bietet sich folgender Aufbau, der sich auch in diesem Text nachvollziehen lässt, an: Zunächst wird der Deutungsmusteransatz nach C. Altmayer vorgestellt. Dem schließt sich eine Erläuterung der eigenständigen Weiterentwicklung dieses theoretischen Modells an. In einem nächsten Schritt werden Herleitung und Zusammensetzung des analytischen Instrumentariums, mit dessen Hilfe kulturelle Deutungsmuster aus Texten herausgearbeitet und rekonstruiert werden können, dargestellt; es folgt eine kurze Skizzierung des Materialkorpus und seiner Textsorten. Ein letzter Schritt präsentiert ein praktisches Textanalysebeispiel, an dem das methodisch-analytische Vorgehen in allen Phasen ausschnittsweise durchexerziert wird; dies soll der zusätzlichen Verdeutlichung des komplexen Verfahrens dienen. 


\section{Die kulturwissenschaftliche Deutungsmusteranalyse}

Im Folgenden wird zunächst der Begriff des kulturellen Deutungsmusters nach C. Altmayer mit den sich daraus ergebenden Merkmalen und Eigenschaftszuweisungen erläutert. Diesem Überblick über die Grundlagen des DeutungsmusterAnsatzes schließt sich eine komplementäre Erweiterung des Ansatzes in elementaren Aspekten an.

\subsection{Der Deutungsmusteransatz nach C. Altmayer}

C. Altmayer leistet mit seinem Deutungsmusteransatz eine kulturwissenschaftliche Transformation der herkömmlichen Landeskunde DaF (Altmayer 2004). Er geht zur begrifflichen Präzisierung seines Kulturbegriffs grundlegend von der erkenntnistheoretischen Prämisse aus, dass Wirklichkeit ein soziales und diskursiv gedeutetes Konstrukt ist. Die gemeinsame Wirklichkeit wird ,auf der Basis von Mustern [gedeutet], die wir in der Regel [...] als selbstverständlich voraussetzen, die aber auch $[\ldots]$ zum Gegenstand diskursiver und kontroverser Deutungsprozesse werden können“ (Altmayer 2006: 51). Gelten diese Muster als ,,̈̈berlieferte, im kulturellen Gedächtnis einer Gruppe gespeicherte und abrufbare Muster von einer gewissen Stabilität" (ebd.), handelt es sich um kulturelle Deutungsmuster. Demzufolge definiert Altmayer die Kultur einer Gruppe als „den Bestand an kulturellen Deutungsmustern, der einer Gruppe als gemeinsamer Wissensvorrat für die gemeinsame diskursive Wirklichkeitsdeutung zur Verfügung steht" (ebd.). Diese Muster sind im „kollektiven Wissensfundus einer Gruppe vorhanden und dort textuell gespeichert und überliefert“ (ebd.: 52). Sie fungieren als „eine Art offener Fundus“, der „mehrere unterschiedliche und teilweise auch miteinander konkurrierende Muster bereithält" (ebd.: 53). Zudem können hier jederzeit neue Muster eingeführt oder ,„̈ltere, in tieferen Schichten des kulturellen Speichergedächtnisses abgelagerte Muster aktualisiert werden“ (ebd.).

Dabei existieren sie laut Altmayer auf zwei verschiedenen strukturellen Ebenen: (1) Auf textueller und diskursiver Ebene als präsupponierte Wissensbestände, und (2) auf der Ebene konkreter und empirischer Verstehensprozesse als verfügbare Interpretationsmuster zur Sinnzuschreibung (Altmayer 2006: 54). Die vorliegende Arbeit fokussiert ausschließlich auf die erstgenannte Ebene und beschäftigt sich infolgedessen mit den Möglichkeiten der Deutungsmusteranalyse in diesem Bereich.

Eine der Hauptaufgaben liegt nun darin, den vorgestellten DeutungsmusterAnsatz im Folgenden um zusätzliche Aspekte zu erweitern, die seinem dynamischen Charakter Rechnung tragen und eine präzisere Beschreibung des Begriffs Deutungsmusters an sich möglich machen. Damit wird ebenso der Praktikabilität einer methodischen Erfassung zugearbeitet. 


\subsection{Weiterentwicklung des DM-Ansatzes}

In meinem Forschungsvorhaben gehe ich zur Weiterführung des oben vorgestellten Ansatzes davon aus, dass ein Deutungsmuster aus einem hypothetischen Fundus heterogener Versatzstücke von Wissen besteht, die ich hier als Bedeutungsvarianten bezeichnen werde.

Diese Varianten lassen sich lediglich anhand von Texten oder Diskursen untersuchen und differieren hinsichtlich ihres textuellen Vorkommens voneinander in Quantität und Qualität, d.h. Durch die Frequenz ihrer Verwendung und durch ihre inhaltlichen Setzungen. Sie enthalten zum Teil sehr unterschiedliche interpretatorische Füllungen und bilden die Bausteine eines Deutungsmusters. Dabei lässt sich ein Deutungsmuster nicht durch scharfe Grenzziehung auf eine genaue Anzahl von Bedeutungsvarianten einschränken, denn es verfügt über keine klare Grenze.

Die Bedeutungsvarianten unterteilen sich in Bedeutungskomponenten und Bedeutungsdominanten, wobei diese Unterscheidung lediglich aus einer Beschäftigung mit der aktuellen Umsetzung im Text getroffen werden kann.

Die Bedeutungsvarianten, die sich durch inhaltliche Dichte und hohe Frequenz im Diskurs auszeichnen, definiere ich hier als Bedeutungsdominanten; sie konstituieren einzelne Diskursstränge und beeinflussen den diskursiven Aushandlungsprozess maßgeblich. Unter Bedeutungskomponenten verstehe ich eben jene Bedeutungsvarianten, die im Diskurs weder durch spezielle inhaltliche Wertigkeit noch durch besondere Häufigkeit des Vorkommens auffallen. In ihrer Formation bilden Bedeutungsdominanten und -komponenten jene präsupponierten Wissensbestände, die dem Text implizit zugrunde liegen.

Aus dieser Setzung erklärt sich der heterogene, bisweilen sogar widersprüchliche Charakter des Deutungsmusters: Es verfügt über ein bestimmtes fluktuierendes Set an Bedeutungsvarianten als eine Art dynamisches Repertoire. Dieses Repertoire scheint sich im Laufe der Zeit zu verwandeln; hier wird jedoch die Annahme vertreten, dass es sich im Laufe der Zeit nicht vollständig verändert, sondern lediglich eine Verlagerung der Wertigkeit innerhalb des BedeutungsvariantenSets stattfindet.

Es erfährt Modifikationen, indem seine Bedeutungsdominanten und Bedeutungskomponenten wechseln: Bestimmte Bedeutungsdominanten werden durch andere ersetzt; d.h. dass je nach zeitgeschichtlichem, gesellschaftlichem oder diskursivem Kontext andere Bedeutungsvarianten textuell betont werden. Die Aufteilung in Komponente und Dominante erklärt, warum kulturelle Deutungsmuster über gewisse Stabilität verfügen und dennoch Bedeutungsveränderungen unterliegen; gleichzeitig liefert sie eine Begründung für die partielle Renaissance älterer, bereits ,verschütteter" Muster, die, in das Bedeutungsvarianten-Set integriert, wieder zur Geltung kommen.

An dieser Stelle muss ergänzt werden, dass sich die Gültigkeit der vorgestellten Modellerweiterung auf Zustandsaufnahmen aus einem Diskursabschnitt bezieht; 
ein vollständiges, zeitloses Deutungsmuster abzubilden ist aus dem geschilderten Modellverständnis heraus konsequenterweise unmöglich.

Daraus ergibt sich der Anspruch an das analytische Instrumentarium, eine Annäherung an die Komplexität des Musters zu gewähren; es soll auch ein Erfassen früherer Bedeutungskomponenten ermöglichen, um gegebenenfalls Bedeutungsverlagerungen festzustellen und zu erklären.

\section{Die Verbindung mit der Wissenssoziologischen Diskursanalyse}

Die Wissenssoziologische Diskursanalyse ist eine unter diversen möglichen Forschungsperspektiven aus dem Bereich der qualitativen Sozialforschung. Sie lässt sich in ihrer methodischen Umsetzung in der sozialwissenschaftlichen Hermeneutik verorten (Keller 2004: 7ff..; Keller 2005: 175ff.). Dabei muss betont werden, dass sie gerade keine neuartige Methodik für sich etabliert, sondern auf qualitative Methoden zurückgreift und diese systematisch einbezieht, wobei hier Modifikationen nötig sein können (Keller 2004: 10f.).

Auf dieses methodische Vorgehen nimmt auch die Deutungsmusteranalyse Bezug und versucht analog dazu ein adäquates methodisches Design zu erstellen. Dies begründet sich aus gemeinsamen Grundannahmen von Wissenssoziologischer Diskursforschung und Deutungsmuster-Ansatz hinsichtlich Wissensmustern und ihrer Rolle in Diskursen (Scheufele 2003: 42ff.). Beide Forschungsperspektiven gehen davon aus,

- dass Wissen in Form von Mustern organisiert wird, die sich im Gebrauch von Sprache manifestieren;

- dass Wissen in Diskursen prozessiert, aktualisiert und modifiziert wird, und dass Diskurse signifikante Elemente enthalten, in denen diskursive Wissensmuster zur Geltung kommen.

Dieses implizite Wissen dient zur interaktiven Erzeugung von Bedeutungsstabilisierung oder -veränderung innerhalb einer Diskursgemeinschaft. Daher sehen beide Forschungsrichtungen ihre Aufgabe im Formulieren von Aussagen über die Struktur des kollektiven Wissens einer Gesellschaft (ebd.).

Zusätzlich liegt beiden ein dynamisches Kulturverständnis zugrunde: Kultur funktioniert als „Diskursfeld“ (Keller 2003: 284ff.), in dem Verhandlungen um Bedeutungszuschreibungen stattfinden. Beide Forschungsperspektiven gehen zudem von einer netzartigen Verschränkung des kulturellen Wissens aus. Damit bieten sich für das analytische Verfahren ähnliche Vorgehensweisen an, nämlich prozessorientierte Untersuchungen von Netzwerkstrukturen. 
Trotz der genannten theoretisch-methodologischen Berührungspunkte müssen hier aber auch andere intentionale Schwerpunkt- und Zielsetzungen betont werden:

Die Wissenssoziologische Diskursanalyse betont im Gegensatz zur Deutungsmusteranalyse „die Rolle sozial konstituierter Akteure in der gesellschaftlichen Produktion und Zirkulation von Wissen“ (Keller 2005: 1). Sie ,reorientiert die Diskursperspektive weg von der Frage nach Sprache und hin zum soziologischen Interesse an der Untersuchung gesellschaftlicher Wissensverhältnisse und Wissenspolitiken, zur diskursiven Konstruktion der Wirklichkeit als materialem Prozess“" (ebd.). Die Konzentration richtet sich hier auf Manifestationen von Diskursordnungen in sozialer Wirklichkeitskonstruktion, nicht auf sprachliche Strukturen. Ebenso geht es um die Möglichkeiten von Machtausübung einzelner Aktanten im Kontext von Wissensproduktion und -verbreitung. Die kulturelle Deutungsmusteranalyse dagegen beschäftigt sich mit kulturbezogenem Wissen, das schriftlich verankert und demzufolge textuell zu untersuchen ist. Kulturelle Deutungsmuster werden als textuell präsupponierte Wissensbestände verstanden, die sich zuweilen auf, zuweilen unter der Textoberfläche ansiedeln. Dieses Verständnis von Deutungsmustern schließt die Untersuchung von Prozessen der Wissens- oder Informationsaufnahme und -auswirkung auf Aktantenseite aus.

Daraus lässt sich schlussfolgern, dass die wissenssoziologischen Einzelbausteine zwar für die Deutungsmusteranalyse übernommen werden können, jedoch anderen Zielorientierungen gemäß arrangiert werden müssen.

\section{Vorstellung des Materialkorpus}

Das Materialkorpus wurde aus Artikeln, Karikaturen und Bildern aus den Printmedien, die sich mit dem Thema EU-Beitritt der Türkei beschäftigen, zusammengestellt: ZEIT, SPIEGEL, FAZ, BILD und STERN. Die Medienwahl richtet sich nach der jeweiligen Auflagenstärke und bot sich zudem wegen der qualitativen Streuung (Boulevardpresse - „,seriöse“ Zeitungen) an. Dabei wurde der Zeitraum von Mitte 2002 bis Ende 2004 als Untersuchungsfeld angesetzt: Im Dezember 2002 fand das Gipfeltreffen von Kopenhagen statt, auf dem man vereinbarte, im Dezember 2004 endgültig über den Kandidatenstatus der Türkei zu entscheiden. In diesem Zeitraum lässt sich ein deutliches Ansteigen der Zahl der Beiträge verzeichnen. Es handelt sich hier um ca. 1.000 Texte, die entweder in Form von Online-Archiven, Sichtung der Zeitungen/Zeitschriften selbst oder mit Hilfe von speziellen Dossiers geprüft wurden. Dieser Korpusabschnitt wird ergänzt durch das Internet-Leserforum zur ZEIT- Debatte: „EU-Beitritt der Türkei“, wobei sich der Untersuchungszeitraum ebenfalls auf 09.2002-12.2004 bezieht; hier sind es 1158 unterschiedlich umfangreiche Beiträge. 


\subsection{Selektive Aufteilung des Korpus in verschiedene Textsorten}

Nach der Durchsicht aller Beiträge wurden aus diesem Gesamtkorpus Einzeltexte extrahiert; dies geschah nach einer Zuteilung in Schlüsseltexte und Korrektivtexte. Darüber hinaus werden Hintergrundtexte außerhalb des eigentlichen Korpus' hinzugezogen. Diese Unterscheidung war notwendig, um das Korpus in reduzierter Form handhabbar zu machen. Sie richtete sich nach folgenden Kriterien:

- Schlüsseltexte: Als Schlüsseltexte wurden die Texte gefasst, die über eine hohe diskursive Relevanz verfügen, d.h. denen diskursintern eine überdurchschnittliche Bedeutsamkeit zugestanden wird; sie erörtern grundlegende inhaltliche Kernpunkte des Diskurses. Dazu trägt auch das symbolische Kapital des Autors bei, der in den Medien meist als Fachmann zu einem Thema gehandelt wird und deswegen innerhalb des Diskurses häufig auftritt. Als Summe der Schlüsseltexte wurden ca. 25 Texte festgesetzt.

- Korrektivtexte: Unter Korrektivtexten verstehe ich aussagekräftige Texte des Internetforums und der Printmedien, die jedoch inhaltlich weniger gehaltvoll als Schlüsseltexte sind, d.h. sie erreichen qualitativ nicht deren diskursinterne Bedeutsamkeit; auch die Autoren verfügen meist nicht über hohes symbolisches Kapital. Die Summe der Korrektivtexte beträgt ca. 40-50 Texte.

- Hintergrundtexte: Als Hintergrundtexte bezeichne ich hier die so genannten kanonischen Texte, die das Deutungsmuster selbst zum Gegenstand haben und diesbezüglich Standards gesetzt haben. Gleichzeitig verfügen sie über einen gewissen Bekanntheitsgrad und dementsprechend über eine längere Rezeptionsgeschichte, die die Rezeption des Deutungsmusters geprägt hat. Diese Definition lehnt sich an die gleich lautende begriffliche Bestimmung C. Altmayers (2004: 260 ff.) an.

Im Gegensatz zu den beiden erstgenannten Textsorten existiert dieser Textkorpusteil nicht real, sondern quasi virtuell; er muss im Laufe der empirischen Untersuchung erst zusammengestellt werden. Ich gehe davon aus, dass ein Hintergrundtext sich aus Intertextualitätsmarkierungen im Schlüsseltext aufspüren lässt. Dabei verstehe ich Intertextualitätsmarker als direkte und indirekte, d.h. Wörtliche und stark sinngemäße textinterne Verweise auf einen dem Text zugrunde liegenden Hintergrundtext. Diese Markierungen lassen sich im Zuge der Schlüsseltextanalysen herausfiltern; anschließend kann ihnen nachgegangen werden. Aufgrund der geschilderten Verfahrensabfolge stehen also die Schlüsseltextanalysen an erster Stelle; demzufolge können zu diesem Zeitpunkt kaum Aussagen über die Summe der Hintergrundtexte gemacht werden. Als sicher kann lediglich gelten, dass ein ausführliches Einbeziehen aus zeitlichen Gründen nur für 4-5 Texte geleistet werden kann. 


\section{Das methodische Vorgehen im Überblick}

\subsection{Die Schlüsseltextanalyse}

Begonnen werden soll hier mit der ersten Analysephase, der Schlüsseltextanalyse, die nur auf die Schlüsseltexte angewendet wird. Sie besteht aus drei Schritten, nämlich zuerst der Makroanalyse, anschließend der Mikroanalyse und zuletzt aus dem Kodierverfahren mit anschließender Konzeptbildung. Ziel ist das Herausarbeiten von Konzepten, die zu „,vorläufigen Bedeutungsdominanten“ komprimiert werden.

\subsubsection{Die Makroanalyse}

Nach kurzem Vorstellen des Textsortenmusters (Vorstellen von Medium, Titel, Autor, Datum, Textsorte) setzt die Makroanalyse ein, die sich v.a. mit dem Nachvollziehen indirekter sinnhafter Verbindungen unter der Textoberfläche beschäftigt, um sich inhaltlichen Tiefenstrukturen anzunähern. Dies geschieht durch Anwendung diskursanalytischer und interpretativer Instrumentarien: Zunächst wird das innere Konstruktionsgerüst des Textes mit Hilfe der Phänomenstrukturanalyse nach Keller „nachgebaut“ (Keller 1998: 232). Dazu dienen folgende Leitfragen:

- Welches Phänomen wird in dem Text entworfen?

- Welche Konsequenzen zieht das nach sich?

- Was wird als Lösungsstrategie vorgeschlagen, welche Wertebezüge werden vermittelt?

Anschließend folgt eine Argumentationskettenanalyse, die indirekte argumentative Verbindungen und unausgesprochene intratextuelle Schlussfolgerungen verdeutlicht. Der Fokus liegt auf folgenden Fragen:

- Welche Verbindungen werden hier aufgetan?

- Welche impliziten Ergänzungen werden bei Schlussfolgerungen vorgenommen?

- Wann und in welcher sprachlichen Form werden welche Argumente wiederholt eingesetzt? Dabei greife ich auf S. Toulmins Argumentationsschema zurück, allerdings auf seine von M. Pielenz erweiterte Form, die leichter handhabbar und begrifflich klarer gefasst ist (Pielenz 1993: 38ff.).

Danach werden einzelne Diskursstränge, die den Text durchziehen, nachvollzogen und festgehalten. Ziel ist es, nach dem Abschluss der Schlüsseltextanalysen Aussagen über die Frequenz der Verbindungen von Argumentationsketten, Deutungsmustern und einzelnen Diskurssträngen machen zu können. 


\subsubsection{Die Mikroanalyse}

Die Mikroanalyse greift v.a. auf linguistisches Instrumentarium zurück, um sich sprachlich-semiotischen Bedeutungsfeldern auf der Textoberfläche zu nähern. Ich gehe davon aus, dass diese bereits Hinweise auf inhaltliche Bedeutungsspeicher enthalten. Dabei interessieren v.a. Metaphernkonzepte, Personifikationen und rhetorische Figuren wie Vergleiche oder Bilder; aber auch Implikaturen, Induktionseffekte und Kollokationen. Hinsichtlich der letztgenannten Punkte wurde die Mikroanalyse von S. Schiffers analytischem Prozedere angeregt, ohne gänzlich übernommen worden zu sein (Schiffer 2004). Ein letzter Schritt stellt gegebenenfalls Intertextualitätsmarkierungen heraus, die sich im Text eingelagert haben.

\subsubsection{Das Kodierverfabren}

Das Kodierverfahren besteht in der Zuweisung von Kategorien zu den Schlagwörtern Türkei, Deutschland, Europa und EU. Zu den genannten Schlagwörtern werden nun Ankerbeispiele aus dem Text, die Syntagmen, herausgesucht und in einer Tabellenform untereinander aufgelistet (hier rechte Tabellenspalte). Aus diesem Material werden induktiv so genannte Kategorien gebildet, die Oberbegriffe; diese werden durch die Beifügung von Slots, d.h. Prädikativen Zuweisungen, in ihrer Bedeutung zusätzlich aufgefächert.

Aus Syntagmen, Slots und Kategorien wird ein verdichtetes Konzentrat der gemeinsamen Inhalte in eigenen Worten erstellt, das so genannte Konzept (hier linke Tabellenspalte). Nachdem mehrere Schlüsseltextanalysen ähnliche Konzepte zum Ergebnis hatten, werden diese auf eine abstrakte Metaebene gehoben und zur vorläufigen Bedeutungsdominante komprimiert.

Das Kodierverfahren bedient sich also eines qualitativen methodischen Instrumentariums, um die inhaltliche Bedeutungsspannbreite nicht künstlich einzuschränken, sondern um einen offenen Textzugang zu inhaltlichen Wissensbeständen zu garantieren. Das Hauptanliegen besteht auch hier darin, sich den Mustern adäquat unter Wahrung der Intersubjektivität und Transparenz zu nähern. Zusätzlich wird nach dem Prinzip minimaler und maximaler Kodierung aus der Grounded Theory gearbeitet (vgl. Böhm 2000: 475).

\subsection{Die Korrektivtextanalyse}

Die Korrektivtextanalyse stellt die zweite Phase (Validierungsphase) des analytischen Vorgehens dar und fungiert als „Testdurchlauf“ für die vorläufigen Bedeutungsdominanten, die sich hier „bewähren“ müssen. Die in den Schlüsseltextanalysen herausgearbeiteten vorläufigen Bedeutungsdominanten agieren hier als Kategorien der Kodierung. Mit ihrer Hilfe werden die ausgesuchten Korrektivtexte kodiert, indem Textbeispiele den Kategorien, d.h. Den vorläufigen Deutungsmustern, 
zugeordnet werden. Ergeben sich daraus neue Bedeutungsvarianten, wird die vorläufige Bedeutungsdominante abgeändert und damit weitergearbeitet.

Ziel ist die Überprüfung und gegebenenfalls die Modifikation der Kategorien. Bei Erreichen einer gewissen Sättigung, d.h. Wenn das Kodierverfahren die Kategorien nur noch bestätigt, wird kein Material mehr herangezogen, und die vorläufigen Bedeutungsdominanten können bestätigt werden. Gemäß der vorgenommenen Begriffsfassungen gehe ich nun davon aus, dass diese in dem hier vorliegenden Diskursausschnitt den Status von Bedeutungsdominanten einnehmen; dies gilt allerdings nur für das untersuchte Fragment des eingegrenzten medialen Diskurses. Die Bedeutungsdominante des Deutungsmusters soll im Folgenden um weitere Komponenten ergänzt werden. Diese werden aus den Hintergrundtexten herausarbeitet.

\subsection{Hintergrundtextanalyse}

Bei der Hintergrundtextanalyse handelt es sich um die dritte Analysephase, deren Aufgabe in der Ergänzung liegt. Mit Hilfe der bereits genannten Intertextualitätsmarker sollen Hintergrundtexte gefunden und zur Analyse herangezogen werden. Nur die Marker werden beachtet, die sich auf textkonstituierende inhaltliche Felder beziehen. Anschließend muss der Hintergrundtext geprüft werden, ob er zur Analyse geeignet ist bzgl. Seiner Ergiebigkeit, des Umfang seiner Rezeptionsgeschichte und der Standardsetzung hinsichtlich des Deutungsmusters.

Mit traditionellen text- und inhaltsanalytischen Mitteln werden nun Textpassagen untersucht, die das vorläufige Deutungsmuster zum Gegenstand haben. Ziel des Vorgehens ist es, mit Hilfe des Hintergrundtextes weitere Bedeutungskomponenten ausfindig zu machen. Um dem Vorwurf der subjektiven Beliebigkeit zu entgehen, wird die Kontextualisierung mit den Hintergrundtexten immer streng vom Material ausgehend vorgenommen. Daher resultiert auch die strikte Orientierung an den Intertextualitätsmarkern.

\subsection{Die Phase der Synthetisierung: Rekonstruktion des Deutungsmusters}

In dieser vierten und letzten Phase erfolgt eine Verbindung von Bedeutungskomponenten und -dominanten, die damit zu einem Deutungsmuster zusammengefügt werden. Dieses soll anschließend inhaltlich in seinen unterschiedlichen Bedeutungsvarianten möglichst ausführlich expliziert werden. Über die Wertigkeitsverlagerung von Bedeutungskomponenten zu Bedeutungsdominanten, die innerhalb des Fundus von Bedeutungsvarianten stattfinden, sollen Aussagen über die inhaltliche Stabilität und das Wandlungsvermögen der jeweiligen Bedeutungsvarianten möglich werden. Daraus leiten sich auch Schlussfolgerungen ab, was der jeweilige Wandel für den aktuellen Diskurseinsatz des Deutungsmusters mit sich bringt. Das rekonstruierte Deutungsmuster wird in seinem diskursiven Kontext gezeigt: In welchem Diskursstrang spielt dieses Muster eine Rolle, wie wird es strategisch 
eingesetzt, welche Funktion übernimmt es für den Diskurs? An dieser Stelle soll nochmals darauf verwiesen werden, dass es sich bei der Rekonstruktion eines Deutungsmusters um einen reinen Annäherungsprozess an ein gedankliches Forschungskonzept handelt.

\section{Das methodische Vorgehen anhand einer exemplarischen Schlüsseltextanalyse}

Um die oben beschriebene Vorgehensweise zu verdeutlichen, soll sie hier anhand eines praktischen Beispiels durchgespielt werden. ${ }^{\mathrm{i}}$

\subsection{Die Schlüsseltextanalyse (Gekürzte Version)}

Titel: Die türkische Frage. Unterzeile: Europas Bürger müssen entscheiden

Autor: Prof. Dr. Hans-Ulrich Wehler, Zeitung: FAZ, Datum: 19.12.2003, Nr. 295, Seite 35

Begründung Schlüsseltext:

- inhaltliche Relevanz: Vorgängerartikel und Pendant zu „Die europäische Antwort“ von W. Burgdorf vom 06.01.2004; Ausführungen zu relevanten Themengebieten

- symbolisches Kapital des Autors: Renommierter Professor für deutsche Sozialgeschichte Uni Berlin/ Bielefeld, prominenter Gegner des Beitritts.

Ressort: Feuilleton

Peritext: reiner Text, in zwei gleichgroße Spalten gegliedert; jede Spalte ist einmal durchbrochen mit einer eingefügten Zwischenunterschrift: 1. „Uneingelöstes Eheversprechen“ und 2. „Fragwürdiger Gewinn“.

Textmuster/ Genre: Glosse/ Kommentar

\section{MAKROANALYSE}

Phänomenstruktur:

Phänomen/ Kernproblem:

Die Türkei hat noch nie zu Europa gehört, deswegen gehört sie auch jetzt nicht in die EU. Sie muss zum islamisch-kleinasiatischen Kulturkreis zugeordnet werden, der weder religiöskulturell, historisch, noch wirtschaftlich mit dem europäischen Kulturkreis vereinbar ist.

Kontext/ Ursache: In einem knappen Jahr wird die EU-Prüfungskommission über den Beitrittswunsch der Türkei entscheiden. Die deutsche Regierung hat sich aus moralischen Gründen (Anschläge in der Türkei) für eine Beitrittsbefürwortung entschieden; dabei wird die Diskussion unsachlich und emotional geführt (275-277). 
Konsequenzen: Wenn es aufgrund der unsachlich geführten Debatte tatsächlich zu einem EUBeitritt der Türkei kommen sollte, würde dies Europa stark beschädigen, vielleicht sogar vernichten: In finanzieller Hinsicht (170-200), gerade hinsichtlich des Wirtschaftsmigrantenansturms (201-219); aber auch in außenpolitischer Hinsicht (riskante geostrategische Lage, ungelöste Binnenkonflikte der Türkei) und aus innenpolitischen Gründen (Steigerung der Europaskepsis bei den Bürgern, Ablehnung der EU).

Handlungsstrategie/Problemlösung: „Europas Bürger müssen entscheiden“ (Untertitel): Europas „mündige Bürger“ müssen im Europa-Wahlkampf über den Türkei-Beitritt entscheiden; sie werden als aufgeklärte Wähler noch unvorbelastet für ein Nein stimmen, im Gegensatz zu den opportunistischen Parlamenten (275-277).

Wertebezug: Entwurf von zwei streng getrennten „Kulturkreisen“: der „europäische Kulturkreis“ und der „islamisch- kleinasiatische“ Kulturkreis. Eine Vermengung zwischen beiden wäre künstlich; die Aufnahme des türkischen Staates würde den europäischen Kulturkreis beschädigen. Europa ist ein exklusiver Kreis, zu dem man naturgemäß dazugehört - oder eben nicht.

Argumentationskettenanalyse (hier nur in Auszügen)

- (30-36) Aufnahmezusage an die Türkei seit 1964: Die Türkei hat sich nicht bemüht, europakompatibler zu werden $\rightarrow$ die Türkei ist eben nicht europäisch, sondern muss sich darum bemühen, es zu werden. Natürlich gelingt es ihr nicht, und sie hat auch kein Interesse daran, denn eigentlich widerstrebt das ihrem Wesen.

- (74-78) Die Türkei ist kein verlässlicher geostrategischer Gegner, wenn es gegen einen anderen muslimischen Staat geht $\rightarrow$ sie gehören zum selben Kulturkreis; die Türkei würde im Zweifelsfall ihren eigenen und nicht den europäischen Kulturkreis unterstützen.

- (120-121) Kulturell-zivilisatorische Grenze: Diese kulturelle Grenze hat auch etwas mit Zivilisation als Entwicklung zu tun $\rightarrow$ Die europäischen Staaten sind auf einem höheren Niveau, dem der islamische Kulturkreis bisher vergeblich nacheifern (trotz der Aufholjagd seit Atatürk); der islamische Kulturkreis ist rückständig und unterentwickelt.

- (160-166) Islam und antiwestlicher Fundamentalismus: Der Islam steht gerade dem Christentum aggressiv und feindselig gegenüber $\rightarrow$ Die Türkei als muslimisches Land impliziert eine latente Bedrohung für das christlich geprägten Europa.

- (166-169) Mangel an innerislamischen Reformen $\rightarrow$ für die dringend notwendige Aufklärung steht wieder das europäische Vorbild; der Türkei fehlt die geistige Kapazität und Durchsetzungsvermögen, eine solche Reformation nachzuahmen. Sie bleibt in rückständigen religiösen Strukturen gefangen $\rightarrow$ der Islam ist nicht reformierbar, denn er ist per se rückständig und statisch. 
FEINANALYSE/ MIKROANALYSE (ebenfalls gekürzt)

\section{Stilmittel:}

Rhetorische Fragen: Um wen anders als um muslimische Fragen wird es aber bei zukünftigen Nahost-Konflikten gehen? (77-81) $\rightarrow$ Implizite Antwort: Der Islam verursacht Konflikte und kriegerische Auseinandersetzungen.

Ironie: „Multikulti-Gutmenschen“ (122), ,,es gilt derzeit nicht als schick“ (119-120); „Warum nur sollte sich Europa so attraktive Nachbarn wie ....machen?" (220-228) $\rightarrow$ Hinweise auf Sinnlosigkeit und Realitätsfremde des Diskussionsverlaufs

Metaphorische Konzepte/ Bilder/ Implikatoren:

Konzept „Eheversprechen“:

Ursprüngliche Zusage der EU als Eheversprechen an die Türkei, die jedoch keine Anstrengungen gemacht hat, um sich dieser Vereinigung als würdig zu erweisen, das heißt: sich anzugleichen. So, wie sie jetzt ist, hat sie diese Verbindung nicht verdient; deswegen ist das Versprechen an sich hinfällig (28-43). Der Türkei wird der feminine, passive Part zugewiesen; sie ist auf diese Verbindung stärker angewiesen, trägt jedoch trotz ihrer Abhängigkeit Schuld am Scheitern aus Mangel an Vermögen und Willen.

Konzept „Islam als bedrohliche Flut“:

In der Türkei existiert eine bedrohlich anschwellende Strömung des Islamismus (123-127) und eine dogmatisch verankerte Feindschaft gegen den Westen (154-156); dazu kommen neue Auswanderungswellen (10-18 Mio. verarmter Anatolier) (205-210) $\rightarrow$ Bild bedrohlicher fundamentalistischer Massen, die den Westen überschwemmen, wird entworfen $\rightarrow$ der Islam als eine fanatische, expansionsbegierige Religion; das gilt auch für die Türkei, die dem islamischen Kulturkreis angehört $\rightarrow$ äußere Bedrohung Europas

Assertion: Migrationsproblem; verarmte Anatolier; Wanderungsbewegungen (203-210)

Folge des EU-Beitritts der Türkei: Massen verarmter Anatolier drängen nach Europa; weil ihre Integration unmöglich ist, verschärft sich das interne Integrationsproblem $\rightarrow$ innere Bedrohung

Sinninferenæ: Migrationsproblem, Integration, Wanderungswellen, Augen verschließen (203$210) \rightarrow$ Migranten bringen immer Probleme mit sich, denn sie sind zu fremd, um sich zu integrieren. Sie überschwemmen und schädigen das arglose Aufnahmeland.

Kollokation: häufiges Zusammenauftreten von Islam und der adjektivischen Zuschreibung „islamistisch": islamistische Mehrheitspartei in muslimischem Staat, Islam und fundamentalistisch, Islam und radikal antiwestlicher Fundamentalismus $(54,123-138,194-186) \rightarrow$ der Islam ist als fanatische Religion ein entschiedener Gegner des Westens

Intertextualitätsmarkierungen (in den Syntagmen fettgedruckt):

„Grenze zwischen zwei Kulturkreisen“ (116-117), ,innerhalb enger Grenzen sich wandelnde Größe“ (110-111), „kulturell-zivilisatorische Grenze“ (120-121) $\rightarrow$ Verweis auf Kulturkreisthesen von Oswald Spengler und S.P. Huntingtons Kulturkampfthese 
Kodierschemata zu den Schlagwörtern „Türkei“ und „Europa“ (ebenfalls gekürzt):

(Schlagwort + Kategorie (Slot 1, Slot 2, Slot 3))

Türkei + defizitär (rückständig*, nicht demokratisch*, verarmt*)

\begin{tabular}{|c|c|}
\hline Konzept: & Syntagmen: \\
\hline $\begin{array}{l}\text { Die Türkei ist kein } \\
\text { demokratisches } \\
\text { Land, sondern hat } \\
\text { politisch- } \\
\text { gesellschaftlich und } \\
\text { wirtschaftlich erbebli- } \\
\text { che Mängel; } \\
\text { zu effektiven Refor- } \\
\text { men fehlt ibr der } \\
\text { nötige Wille; auch } \\
\text { traditionell-religiöse } \\
\text { Prägungen verbin- } \\
\text { dern Neuerungen }\end{array}$ & $\begin{array}{l}\text { - }[\ldots] \text { demokratischem Experiment in einem muslimischen Land } \\
\text { wie der Türkei endlich zum Erfolg verhelfen (84-87) } \\
\text { - }[\ldots] \text { nicht durch die Antike, das römische Recht, die Reformation, } \\
\text { geschweige die Aufklärung, nicht durch das okzidentale Bürger- } \\
\text { tum [...] geprägt worden, auch wenn die Aufholjagd seit [...] Ata- } \\
\text { türk anhält (98-109) } \\
\text { - tiefverwurzelte Bräuche wie etwa die europäisches Recht verlet- } \\
\text { zende Zwangsehe im Kinderalter oder [...] Verheiratung junger } \\
\text { Töchter [...] (140-148) } \\
\text { - wo bleibt nur eine innerislamistische Aufklärung oder Reforma- } \\
\text { tion, die sich solcher Probleme endlich annimmt? (166-169) } \\
\text { - die Türkei ist ein Land wirtschaftlicher Rückständigkeit geblie- } \\
\text { ben (170-174) } \\
\text { - [...] archaisch erstarrte Landwirtschaft, meist auf der Basis zwerg- } \\
\text { bäuerlicher Subsistenzbetriebe (179-183) } \\
\text { - }[\ldots . .] \text { vom Makel der Korruption [...] noch lange nicht befreit [...] } \\
\text { (187-191). }\end{array}$ \\
\hline
\end{tabular}

Türkei + Islam (fanatisch*, nicht vertrauenswürdig*)

\begin{tabular}{|c|c|}
\hline $\begin{array}{l}\text { Der Islam ist eine } \\
\text { potentiell fanatische } \\
\text { Religion, die alle } \\
\text { anderen Religionen } \\
\text { ablehnt. Die Türkei } \\
\text { ist durch den Islam } \\
\text { geprögt und gehört } \\
\text { qu einem anderen } \\
\text { Kulturkreis }\end{array}$ & 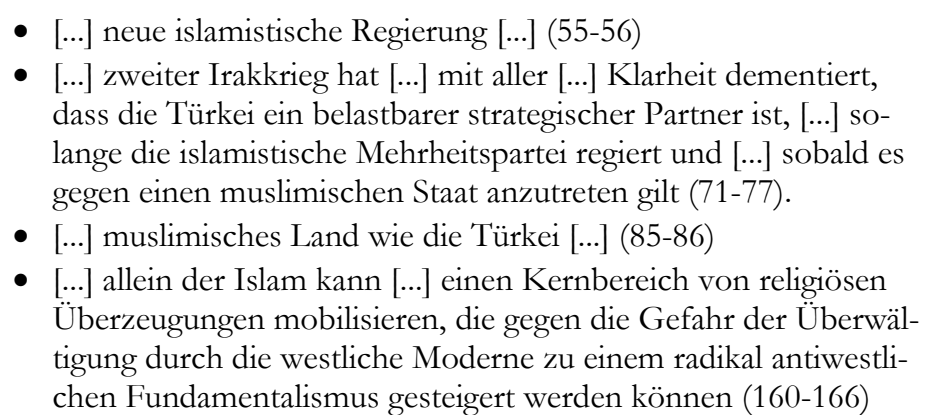 \\
\hline
\end{tabular}


Türkei + Bedrohung (agressiv*, islamistisch*, fanatisch*)

\begin{tabular}{|c|c|}
\hline $\begin{array}{l}\text { In der Türkei findet } \\
\text { eine staatlich unter- } \\
\text { stützte Rückbesin- } \\
\text { nung auf den fun- } \\
\text { damentalistischen } \\
\text { Islam statt. }\end{array}$ & $\begin{array}{l}\text { - }[\ldots] \text { vom türkischen Heer anvisierter Einmarsch [...], um [...] } \\
\text { kurdische Autonomie mit Waffengewalt zu ersticken [...] (64-72) } \\
\text { - }[\ldots . . .] \text { Regierungen in Ankara haben mit Großzügigkeit gegenüber } \\
\text { islamistischen Forderungen, ja mit Kooperation mit der bedroh- } \\
\text { lich schwellenden Strömung regiert (123-127) } \\
\text { - } \text { islamistische Schulen [...] staatlich finanziert (129-130) }\end{array}$ \\
\hline $\begin{array}{l}\text { Die Türkei ist an } \\
\text { sich schon ein poten- } \\
\text { tiell gewalttätiger } \\
\text { Staat mit hohem } \\
\text { Herrschaftsanspruch. } \\
\text { Diese Eigenschaft } \\
\text { wird durch den } \\
\text { Islam verstärkt }\end{array}$ & $\begin{array}{l}\text { - [...] (islamistische Schulen, Anmerkung I.M.) wurden von fünf- } \\
\text { zehn Prozent aller höheren Schüler besucht, und die Absolven- } \\
\text { ten drängen mit Macht in den Staatsdienst (131-134) } \\
\text { - das Kopftuch für Mädchen und Frauen, demonstratives [...] } \\
\text { Symbol orthodoxer Rechtgläubigkeit, wurde weithin wieder er- } \\
\text { laubt (134-138) } \\
\text { - } \quad[. . .] \text { allein der Islam kann [...] einen Kernbereich von religiösen } \\
\text { Überzeugungen mobilisieren, die gegen die Gefahr der Überwäl- } \\
\text { tigung durch die westliche Moderne zu einem radikal antiwestli- } \\
\text { chen Fundamentalismus gesteigert werden können (160-166) }\end{array}$ \\
\hline
\end{tabular}

Türkei + anders / fremd (nicht europäisch*, nicht europakompatibel*)

\begin{tabular}{|c|c|}
\hline $\begin{array}{l}\text { Die Türkei hat noch } \\
\text { nie zu Europa } \\
\text { gehört. } \\
\text { Sie gebört zu einem } \\
\text { anderen, rückständi- } \\
\text { gen Kulturkereis, so } \\
\text { sehr sie sich auch } \\
\text { bemühen mag. }\end{array}$ & $\begin{array}{l}\text { - }[\ldots . .] \text { neurotischer Druck, Kleinasien endlich als ,europäisch“ } \\
\text { anzuerkennen (87-89) } \\
\text { - }[\ldots . .] \text { erst recht die Türkei (ist) [...] nie Bestandteil des historischen } \\
\text { Europas gewesen (98-101). } \\
\text { - }[\ldots . .] \text { nicht durch die Antike, das römische Recht, die Reformation, } \\
\text { geschweige die Aufklärung, nicht durch das okzidentale Bürger- } \\
\text { tum [...] geprägt worden, auch wenn die Aufholjagd seit [...] Ata- } \\
\text { türk anhält (98-109). } \\
\text { - }-[\ldots . .] \text { ein auf ganz andersartiger historischer Basis beruhender } \\
\text { Staat wie die Türkei [...] (250-253) }\end{array}$ \\
\hline $\begin{array}{l}\text { Zwischen der Türkei } \\
\text { und dem Westen } \\
\text { existiert eine un- } \\
\text { überwindbare Kluft }\end{array}$ & $\begin{array}{l}\text { - }[\ldots] \text { in Hinblick auf die Türkei kommt die Grenze zwischen zwei } \\
\text { Kulturkreisen hinzu, die [...] keineswegs überwunden worden ist } \\
\text { (115-119) } \\
\text { - }[\ldots . .] \text { kulturell-zivilisatorische Grenze }(120-121)\end{array}$ \\
\hline
\end{tabular}


Europa + Fortschritt (entwickelt*, überlegen*, Vorbildfunktion*)

\begin{tabular}{|c|c|}
\hline $\begin{array}{l}\text { Europa als histo- } \\
\text { risch gewachsene und } \\
\text { Zivilisation auf } \\
\text { böherem Entwick- } \\
\text { lungsstand mit } \\
\text { Vorbildfunktion für } \\
\text { Kleinasien/Türkei }\end{array}$ & 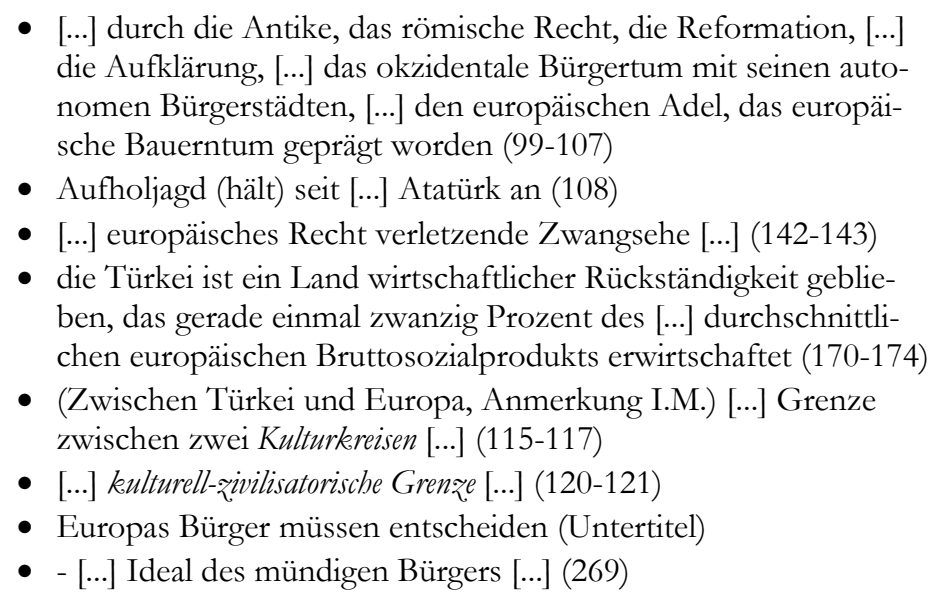 \\
\hline
\end{tabular}

\section{2 Überprüfung durch die Validierungsphase}

Hier schließt sich theoretisch als zweite Analysephase die Korrektivtextanalyse an. Diese kann hier lediglich hypothetisch durchgespielt werden: Da die Schlüsseltextanalysen noch nicht ganz abgeschlossen sind, sind endgültige Formulierungen von vorläufigen Bedeutungsdominanten noch nicht möglich. Um das hier verfolgte Procedere zu verdeutlichen, wähle ich im Folgenden zwei Konzepte aus, an denen exemplarisch das Verfahren gezeigt wird; die hier erzielten Ergebnisse bleiben aber reine Annahmen. Ich gehe also von der wiederholten Bestätigung der oben genannten Konzepte bei den Schlüsseltextanalysen aus. Diese lassen sich auf folgende vorläufige Bedeutungsdominanten komprimieren:

- „Die Türkei ist ein islamisches Land voller Defizite, das zu einem anderen Kulturkreis als Europa gehört."

- „Europa ist eine hoch entwickelte Zivilisation, die einen überlegenen, exklusiven Kulturkreis bildet.“

Sie können nun als Kategorien für die Korrektivanalyse verwendet werden. Der besseren Handhabbarkeit halber kürze ich sie auf die Stichwörter ,die Türkei ist defizitär“, „die Türkei gehört zum islamischen Kulturkreis“ und „Europa ist zivilisiert“, „Europa ist ein exklusiver Kulturkreis“. Mit Hilfe dieser Stichwörter, die als Kodes verwendet werden, lassen sich die Korrektivtexte nun kodieren. Ich gehe nun davon aus, dass die in diesem Kodierverfahren erarbeiteten Konzepte sich ohne Modifikationen in anderen Texten wiederfinden ließen. Damit würden sich die vorläufigen Bedeutungsdominanten als Dominanten im untersuchten Mediendiskurs bestätigen. 


\subsection{Die Elaborationsphase}

Hier würde sich nun die dritte Analysephase, die so genannte Elaborations- oder Ergänzungsphase anschließen. Es geht also darum, aus den Intertextualitätsmarkern einen geeigneten Hintergrundtext aufzuspüren, der für die Rezeption dieses Deutungsmusters Standards gesetzt hat. Aus den Hintergrundtexten sollen relevante Passagen ausgesucht werden, die mit Hilfe textanalytisch-interpretativer Methodik untersucht werden.

Als praktisches Fallbeispiel dazu kann hier eine Textstelle zur so genannten Kulturkreislehre dienen; sie ergibt sich aus der Intertextualitätsmarkierung „Kulturkreis“. Dieses Deutungsmuster findet sich bereits bei O. Spengler in seinem Werk von 1918, Der Untergang des Abendlandes. Seinem Buch ward trotz oder gerade wegen seiner großen Umstrittenheit enorme Popularität zuteil; es erschien 2006 in der 17. Auflage. Spengler skizziert darin die „Umrisse einer Morphologie der Weltgeschichte“ (Untertitel), wobei seine Theorie auf der Gesetzmäßigkeit eines zyklischen Geschichtsablaufes basiert: Da eine gewisse Evolutionsabfolge vorbestimmt ist, muss jede Kultur die dazugehörigen Entwicklungsstufen unweigerlich durchlaufen. Von diesen Hypothesen ausgehend zieht er Vergleiche zwischen früheren historisch-kulturellen Phänomenen und wagt Prognosen über die zukünftige Entwicklung der Welt.

Spengler sieht das Ziel seiner Untersuchung darin, „die organische Struktur der Geschichte bloß[zu]legen“ (Spengler 2006: 6). In der geschichtlichen Entwicklung von Kulturen erkennt er ,[...] Stufen [,] die durchschritten werden müssen, und zwar in einer Ordnung, die keine Ausnahme zulässt [.] Haben die für alles Organische grundlegenden Begriffe, Geburt, Tod, Jugend, Alter, Lebensdauer, in diesem Kreise vielleicht einen strengen Sinn, den noch niemand erschlossen hat?" (ebd.: 3). Seiner Meinung nach existieren die Kulturen auf unterschiedlichem Entwicklungsstand. Die Zivilisation ist die höchste Entwicklungsstufe; sie bildet sich also innerhalb einer Kultur „zu ihrer vollen und endgültigen Gestalt heraus [...] und [beendet] damit die lebendige Entwicklung der Kultur" (ebd.: 614), was dann ihren Niedergang mit sich bringt. Die ,westeuropäisch-amerikanische Kultur“ stellt für Spengler das aktuelle Beispiel dafür dar (ebd.: 3): Er sieht sie in eben diesem Stadium der Dekadenz, kurz vor ihrem Untergang.

Die Kulturen sind laut Spengler relativ streng voneinander getrennte Einheiten, auch wenn gewisse Inhalte voneinander übernommen werden: ,[...] zwischen den Seelen zweier Kulturen [liegt] eine undurchdringliche Scheidewand. [...] Jede versteht nur ein selbstgeschaffenes Bild der anderen [...] (ebd.: 763). Dabei bleibt Spenglers Entwicklungsstufen-Modell wertungsfrei: Wenn jede Kultur sich auf genau dem evolutionären Status befindet, der ihr in der morphologischen Geschichtsentwicklung zukommt, wird damit die Rivalität der Kulturen untereinander obsolet. Eine große Kultur ist als metaphysische Größe gezeichnet, als „etwas ganz Ursprüngliches und aus den tiefsten Gründen des Seelentums Aufsteigendes“ (ebd.: 760). Sie ruft das Volk als Folgeerscheinung hervor und bestimmt dessen 
Verhältnis „zu Rasse, Sprache, Land, Staat, Religion“ (ebd.: 763); nicht jene Parameter bilden ein Volk, sondern vielmehr „etwas Kosmisches und Gerichtetes“ (ebd.: 755).

Diese Kulturkreistheorie wurde mehrfach aufgegriffen; am prominentesten wurde sie in der Bearbeitung S. P. Huntingtons Clash of Civilizations, erschienen 1993. Er geht davon aus, dass das größte kriegerische Potential für das 21. Jahrhundert in dem Zusammenprall verschiedener Kulturkreise liegt. Einen Kulturkreis definiert Huntington als ,[...] die höchste kulturelle Gruppierung von Menschen und die allgemeinste Ebene kultureller Identität des Menschen [...]. Sie definiert sich sowohl durch gemeinsame Elemente wie Sprache, Geschichte, Religion, Sitten, Institutionen als auch durch die subjektive Identifikation der Menschen mit ihr." (Huntington 2006/2007: 56). Auch bei Huntington gibt es die Gesetzmäßigkeit des zyklischen Ablaufs verschiedener Entwicklungsstadien: „Sie [die Kulturkreise, I.M.] sind dynamisch; sie steigen auf und fallen [...]. Die Phasen ihrer Entwicklung kann man auf verschiedene Weise gliedern. [...] alle Theorien [sehen] den Entwicklungsgang von „Zivilisationen“ als den Weg von einer Zeit der Unruhen oder Konflikte über einen Universalstaat bis hin zu Verfall und Auflösung. " (ebd.: 58). Die einzelnen Kulturkreise sind in sich keineswegs homogen, grenzen sich aber dennoch voneinander ab: „Die Kulturen von Völkern wirken aufeinander ein und überlagern sich. [...] Gleichwohl sind Kulturkreise sinnvolle Einheiten, und wenn die Grenzlinien zwischen ihnen auch selten scharf gezogen sind, so sind sie doch vorhanden." (ebd.: 56).

Bis ins 20. Jahrhundert hinein befand sich laut Huntington „der Westen“ in der Rolle des „Universalstaates“, der die globale Weltordnung vorwiegend bestimmt hat. Er bezeichnet ihn als „die erste Zivilisation, die sich modernisierte“ und die „,beim Erwerb der Kultur der Moderne die Führung innehat“ (ebd.: 97). „Der Westen“ umfasse also das, „was man einmal das christliche Abendland zu nennen pflegte. [...] Historisch gesehen ist westliche Kultur europäische Kultur“ (ebd.: 62). Nun lasse die Welt aber allmählich ihre westliche Phase hinter sich, und damit verändere sich das machtpolitische Gewicht:“ [...] die Ideologien [verfallen], die für die späte westliche Zivilisation typisch waren, und an ihre Stelle treten Religionen [...].“ Die Konsequenz: „Die intrakulturelle Auseinandersetzung um die politischen Ideen aus dem Westen wird abgelöst von einer interkulturellen Auseinandersetzung um Kultur und Religion“" (ebd.: 73).

Das Deutungsmuster „Kulturkreis“ erfährt entscheidende Veränderungen: Spengler geht von einer metaphysischen Idee aus, die die Beseelung eines Volkes in dem Sinne erst veranlasst - davor sind Völker lediglich ,flüchtige [...] Verbände, die sich ohne erkennbare Regel [...] bilden und lösen“ (ebd.: 759), bis sie „im Banne der Kultur" erweckt werden und eine seelische Einheit bilden.

Die Kulturkreise generieren sich bei Huntington jedoch umgekehrt aus sozialhistorischen, sprachlich-kulturellen Eigentümlichkeiten von Völkern, und bilden demzufolge von Volksgemeinschaften getragene Einheiten. Konsequenz dieser Umformung ist eine aggressivere Ausrichtung des Modells: Die Kulturkreise liegen 
als feste, determinierende Fundamente den Staaten zugrunde und streben nach Herausbildung und Expansion des machtpolitischen Einflussbereiches. Dadurch werden Auseinandersetzungen wahrscheinlich, fast notwendig. Huntington fügt dem noch eine neue, letztlich explosive Ingredienz hinzu: Die Religion. Sie ist hier das ,elementare[...] Merkmal von Kulturkreisen“: „Von allen objektiven Merkmalen, die eine Kultur definieren, ist jedoch das wichtigste für gewöhnlich die Religion [...]" (ebd.: 54); „Insbesondere die großen missionarischen Religionen, das Christentum und der Islam, umfassen Gesellschaften aus den verschiedensten Rassen. Die wesentlichen Unterschiede zwischen Menschengruppen betreffen ihre Werte, Überzeugungen, Institutionen und Gesellschaftsstrukturen, nicht ihre Körpergröße, Kopfform und Hautfarbe“ (ebd.: 55). Europa selbst wird mit der „westlichen Christenheit" identifiziert (vgl. ebd.: 255); der Islam hingegen bildet einen eigenen Kulturkreis. Demzufolge liegt dem Verhältnis von Islam und Christentum ein kontinuierlicher, auch machtpolitisch ausgetragener Antagonismus zugrunde, denn „die Ursachen für dieses Konfliktmuster entspringen der Natur dieser beiden Religionen und der auf ihnen basierenden Kulturen" (ebd.: 337).

In diesem Kontext erhält die Türkei einen besonderen Stellenwert, und zwar als „das klassische zerrissene Land [Hervorhebung I.M.]“ (ebd.: 217), denn sie hat „eine einzige, herrschende Kultur, durch die es in eine bestimmte Zivilisation gehört, während die Führer des Landes den Wunsch haben, es einer anderen Zivilisation zuzuordnen" (ebd.: 216). Trotz der künstlichen Zwangsorientierung gen Westen setzt sich die indigene Kultur der Türkei durch, die per se prädestiniert ist, den islamischen Kulturkreis als „Kernstaat des Islams“(ebd.: 286 ff.) anzuführen. Dies beweist der wachsende Einfluss des radikalen Islams: „[...] die Resurgenz des Islams in der Türkei [begann], die laizistische, prowestliche Orientierung der Türkei zu untergraben.“ (ebd.: 235). Für Huntington bedeutet die Aufnahme der Türkei in die EU ein unzumutbares Risiko: ,[...] kulturelle Unterschiede [fördern] Spaltungen und Konflikte" (ebd).

Wie oberhalb erläutert wurde, liegt dem Deutungsmuster „Kulturkreis“ bei Spengler ein biomorph-wertneutrales Verständnis von Kultur zugrunde: Jede Kultur basiert auf metaphysischen Grundströmen des Daseins, die einer Gesellschaft erst die seelische Einheit verleihen, die es zum Volk macht. Sie unterliegen ihrerseits der Gesetzmäßigkeit eines zyklischen Entwicklungsablaufes, durch die ihre Lebensdauer als Volksgemeinschaft/ Nation bestimmt ist (Spengler 2006: 762).

Huntington greift die Betonung dieses zyklischen Ablaufmodells wieder auf, wobei machtpolitischen Konstellationen und das Geschick des jeweiligen Kulturkreises den eigenen Status bestimmen; jeder Kulturkreis strebt nach Hegemonialmacht. So erhält das Deutungsmuster eine viel stärker selbst bestimmte, aktivistische Komponente, die durch Hinzufügung der missionarisch-religiösen Prägung noch verschärft wird. Bei Huntington bildet jeder Kulturkreis den natürlichen Antagonisten des anderen im Ringen um machtpolitische Ausdehnung. Ein Volk versteht sich hier durch Berufung auf gemeinsame Geschichte, Sprache und v.a. Religion als ein Kulturkreis. 


\subsection{Schlussfolgerung: Synthetisierungsphase}

Auch diese Phase kann wieder lediglich theoretisch vorgestellt werden; hier erfolgt eine Verknüpfung von den oben herausgefilterten Bedeutungskomponenten mit den Bedeutungsdominanten, die in der ersten und zweiten Phase erarbeitet wurden.

Bei den oben genannten Bedeutungsdominanten „die Türkei ist defizitär“, „die Türkei gehört zum islamischen Kulturkreis“ und „Europa ist zivilisiert“, „Europa ist ein exklusiver Kulturkreis“ zeigt sich, inwieweit Bedeutungsvarianten von Huntington/Spengler übernommen wurden. Tatsächlich ordnet Wehler die Türkei dem islamischen Kulturkreis zu. Beweis dafür ist auch bei Wehler die Resurgenz des Islams, die zeigt, dass sich das Land nicht von seiner indigenen Wesensart lösen kann.

Wehler übernimmt hier Huntingtons Bedeutungsvariante: Wegen dieser Zugehörigkeit zu einem anderen Kulturkreis bedeutete die Aufnahme der Türkei generell eine Gefährdung der EU; verstärkt wird dies durch ihre Zugehörigkeit zum islamischen Kulturkreis, der am wenigsten mit dem europäischen, d.h. Christlichabendländischen Kulturkreis zu vereinbaren ist. Huntingtons Setzung der religiösen Kulturkreisfundierung und verbundenen Rivalität der Kulturkreise untereinander wird von Wehler als Bedeutungsdominante verwendet.

Aber auch die zyklische Entwicklungsstadienabfolge, die Spengler als eine vorbestimmte festhält, wird hier aufgegriffen; mit anderen Komponenten versetzt, nimmt sie eine gänzlich neue Wendung an. So zieht Wehler eine Kausalverbindung zwischen religiöser Ausrichtung und Evolutionsniveau (Wehler 2003: 166-169). Damit wird der Islam zu einer rückständigen Religion, die Fortschritt und Weiterentwicklung be- oder verhindert. Aufgrund der Zugehörigkeit zum islamischen Kulturkreis wird der Türkei ein doppeltes defizitäres Moment zugewiesen: Zum einen impliziert dieser Kulturkreis per se einen niedrigeren Entwicklungsstand im Vergleich zum europäischen; zum anderen bewirkt ihre wesenskonträre Westorientierung eine zusätzliche Schwächung und Zerrissenheit. Bei Wehler fällt auch die Bedeutungsvariante der Gesetzmäßigkeit einer zyklischen Entwicklungsabfolge weg; die Kulturkreise verbleiben statisch auf ihrem Entwicklungsniveau.

Die Bedeutungsdominanten „Europa ist hoch entwickelt“ und „Europa bildet einen eigenständigen Kulturkreis mit Vorbildfunktion“ und „die Türkei ist defizitär“ sowie „die Türkei gehört zu dem islamischen, rückständigen Kulturkreis“ aus dem Mediendiskurs greifen also Bedeutungsvarianten von Huntington auf, setzen sie aber in neue Beziehungen zueinander. Daraus ergibt sich eine andere Bedeutungsdominante, die, im medialen Kontext verwendet, enorme Sprengkraft erhält: Der Beitrittswunsch der Türkei impliziert eine latente Bedrohung der EU. Ihr unnatürliches Aufnahmebegehren ist eine Zumutung, denn sie ist Europa nicht nur wesenhaft völlig fremd, sondern verharrt zusätzlich auf einem niedrigeren Entwicklungsniveau. Ihre Aufnahme muss Europa zwangsläufig schaden, wenn nicht sogar zerstören. 


\section{$7 \quad$ Fazit und Ausblick}

Das dargestellte methodische Verfahren befindet sich in einem noch andauernden Entwicklungs- und Überarbeitungsprozess; daher wird hier abschließend ein Ausblick auf noch ausstehende Arbeitsschritte gegeben.

Durch das erarbeitete Procedere können große Materialmengen aufbereitet und bearbeitet werden. Es gewährleistet zu der Untersuchungsbandbreite auch eine gewisse Forschungstiefe: Nicht nur Textoberflächen, sondern auch inhaltliche Tiefenstrukturen können hiermit erfasst und analysiert werden. Dies geschieht unter Berücksichtigung wissenschaftlicher Gütekriterien wie Transparenz des Vorgehens, intersubjektive Objektivität und Validität.

Als problematisch erweist sich auch die strikte Trennung einzelner Bedeutungskomponenten und -dominanten voneinander; da sich bestimmte inhaltliche Bereiche überlappen, lassen sich scharfe Grenzen bisweilen kaum ziehen. Die künftige Forschungsaufgabe besteht darin, das analytische Verfahren vollständig umzusetzen, um zu fundierten inhaltlichen Aussagen zu gelangen, sprich: sich auf die kulturellen Deutungsmuster im Diskurs zu konzentrieren. Da ich davon ausgehe, dass Deutungsmuster eine diskursstrukturierende Funktion übernehmen können, schließt sich an die inhaltliche Ausführung eine Untersuchung von Funktionen von Deutungsmustern im Diskurs an, wobei ich folgende Hypothesen aufstelle: Deutungsmuster fungieren als:

- Legitimationsnachweis, als eine Art Begründung für einen Diskursstrang;

- Widerlegungsbeweis, um andere Diskurspositionen zu entkräften;

- Bindeglieder zwischen einzelnen Diskurssträngen, indem sie von den einzelnen Diskurssträngen an bestimmten Punkten zu anderen „Aussagemöglichkeiten“ weiterleiten;

- „Weichensteller“, d.h. sie wirken als Veränderungsmodul, und lenken den Diskurs in eine andere Richtung, wobei sie ihm eine neue Zusatzkomponente verleihen und schließlich zu seiner Konstituierung in eine andere Bedeutungsrichtung beitragen.

Vom jetzigen Stand der Arbeit aus betrachtet, kann dieser Bereich jedoch nur als Ausblick formuliert werden; dennoch verspricht er, eine relevante Antwort auf die Frage nach dem diskursinternen Funktionieren von Deutungsmustern geben zu können. 


\section{Literatur}

Altmayer, Claus (2004): Kultur als Hypertext. Zur Theorie und Praxis der Kulturwissenschaft im Fach Deutsch als Fremdsprache. München: Iudicium.

Altmayer, Claus (2006): Kulturelle Deutungsmuster als Lerngegenstand. In: Fremdsprachen lehren und lernen 35, 45-59.

Böhm, Andreas (2000): Theoretisches Codieren. Textanalyse in der Grounded Theory. In: Flick, Uwe et al. (Hrsg.) (2000): Qualitative Forschung. Ein Handbuch. Hamburg: Rowohlt, 470-515.

Huntington, Samuel Phillip (2006/2007): Kampf der Kulturen. Hamburg: Spiegel.

Keller, Reiner (2003): Kultur als Diskursfeld. In: Geideck, Susan \& Liebert, Wolf-Andreas (Hrsg.) (2003), Sinnformeln. Berlin/New York: De Gruyter, 283-305.

Keller, Reiner (1998): Müll-Die gesellschaftliche Konstruktion des Wertvollen. Opladen: Westdeutscher Verlag.

Keller, Reiner (2004): Diskursforschung. Wiesbaden: Verlag für Sozialwissenschaften [Qualitative Sozialforschung; 14].

Keller, Reiner (2005): Analysing Discourse. In: Forum Qualitative Sozialforschung 6(3), [http://www.qualitative-research.net; 20.09.2008].

Pielenz, Michael (1993): Argumentation und Metapher. Tübingen: Narr [Tübinger Beiträge zur Linguistik; 381].

Scheufele, Bertram (2003): Frames- Framing- Framing-Effekte. Wiesbaden: Verlag für Sozialwissenschaften.

Schiffer, Sabine (2004): Die Darstellung des Islams in der Presse. Erlangen/Nürnberg: Ergon.

Spengler, Oswald (2006): Der Untergang des Abendlandes. München: Deutscher Taschenbuch Verlag.

Wehler, Hans-Ulrich (2003): Die türkische Frage. In: Frankfurter Allgemeine Zeitung. Nr. 295, 19.12.2003, S. 35 .

i Dabei können die Analysen nur in stark gekürzter Form gezeigt werden, denn eine vollständige Analyse beläuft sich zur Zeit auf ungefähr 10 Seiten und würde den hier vorgegebenen Rahmen bei Weitem sprengen. Die angegebenen Zeilenzahlen stammen aus dem Artikel; nummeriert wurde nur der Fließtext. 


\title{
Integrierte Textentwicklung in einem Radioprojekt mit fortgeschrittenen DaF-Lernenden
}

\author{
Kristina Peuschel (Leiprig)
}

\begin{abstract}
Im folgenden Beitrag wird eine fachdidaktisch motivierte, explorativ-interpretative Studie vorgestellt, die sich dem sprachlichen Lernen der Fremdsprache Deutsch in Radioprojekten widmet. Der Schwerpunkt des Beitrages liegt auf dem Nachvollziehen von Textentwicklungsprozessen, die sich in mündlichen und schriftlichen Teilprodukt(ion)en von Lernenden zeigen. Der Beitrag soll dazu anregen, Projekte als Lehr-Lernform zu entmystifizieren, sich den Potentialen und Grenzen der Förderung sprachlichen Lernens in Projekten zuzuwenden und dahingehend weitere empirische Untersuchungen zu offenen, Autonomie fördernden Lernformen durchzuführen.
\end{abstract}

\section{Projekte im DaF-Unterricht: Forschungsstand}

Angesichts der Vielzahl von konzeptionellen Vorschlägen und Texten zu Projekten, Projektunterricht oder auch projektorientiertem Unterricht im Kontext Fremdsprachen Lehren und Lernen erstaunt der Mangel an empirischen Arbeiten, die sich mit der Lehr-Lernform Projekt beschäftigen, die spätestens seit der kommunikativen Wende fester Bestandteil pädagogischer, didaktischer und methodischer Diskussionen ist (vgl. Schart 2003a). In der Praxis der Fremdsprachenvermittlung, der Lehrerfortbildung und/oder im Bereich des Lernens mit neuen Medien erfreuen sich Projekte großer Beliebtheit (vgl. Jahn \& Schier 2004; MüllerHartmann \& Schocker-von Ditfurth 2004; Šimunek 2007 u.a.), dennoch ist die Zahl der in Fachpublikationen veröffentlichten Praxis- und Erfahrungsberichte über Projekte im DaF-Unterricht überschaubar (vgl. Peuschel 2007). Weitaus auffälliger ist, dass empirische Arbeiten zu Einzelthemen der Sprachlehrforschung und/oder Fremdsprachen(erwerbs-)forschung für Projekte fast völlig fehlen. Das wissenschaftliche Interesse am sprachlichen Lernen in Projekten erschöpft sich nicht selten in vagen Aussagen zum selbständigen Umgang mit „,nicht didaktisch gefilterter“ Fremdsprache (Dietrich 1995: 256) oder dem Wissen um die Herstellung von (implizit) sprachlichen Produkten im Projekt. Eine Ausnahme bilden hier Reuken \& Hentschel (1991), die sich dem Abbau von Sprechhemmungen in einem universitären Projektseminar zuwenden und dies empirisch begleiten.

Einen wichtigen Beitrag zur empirischen Untersuchung von Projekten als Lehr-Lernform liefert Schart (2003a, 2003b), der die Perspektive der Lehrenden auf Projektarbeit bzw. Projektunterricht ausführlich betrachtet. Er kommt zu dem 
Schluss, dass das Thema „empirische Forschung weitaus nötiger [hat] als weitere theoretische Erörterungen [...]“ (Schart 2003b: 576). Šimunek (2007) widmet sich zwar dem sprachlichen Lernen in einem als Projekte definierten Lernkontext, speziell der formelhaften Sprache in Telekollaborationsprojekten im Englischunterricht, allerdings bleibt das Projektspezifische des Lernens nur wenig betrachtet. Es bleibt zu hoffen, dass die aktuell zu beobachtende Annäherung von „Projekten“ an Begrifflichkeiten wie „Lernszenarien“ (vgl. Rasch 1997; Piepho 2003), Lernarrangements oder „Aufgabe“ (vgl. Funk 2006; Legutke 2006) in größerem Maße die empirische Perspektive auf diese Lehr-Lernform ermöglicht.

Die Variabilität sprachlicher Lernprozesse in Projekten muss zunächst explorativ-interpretativ bearbeitet werden, um dann in detaillierten Analysen empirisch abgesichert zum tatsächlichen Potential von Projekten zu gelangen. Dafür kann es hilfreich sein, spezifische Textproduktionsprozesse in Projekten kontextuell eingebettet zu betrachten und nachvollziehbar zu machen.

\section{Authentizität als Echtheit fremdsprachigen Lernens}

Im Gegensatz zur traditionellen und stark verkürzten Auffassung des Gegensatzpaares ,authentische vs. didaktisierte Texte“ und einer einseitig positiven Bewertung der einen oder anderen folge ich der Aussage von Schart, dass ausschlaggebend für den Einsatz von mehr oder minder authentischen Materialien im Fremdsprachenunterricht vor allem das ist, ,was Lernende mit den Unterrichtsmaterialien - gleich welchen Ursprungs - tun, und wie ihr Einsatz motiviert ist [...]“ (Schart 2003a: 94). Authentizität heißt nicht nur ,,authentisches Erleben von Kommunikation im Fremdsprachenunterricht" (ebd.: 96) sondern auch das Erleben inhaltlicher Relevanz auf Lernerseite.

Die von Gudjons (1994: 70) eingeforderte gesellschaftliche Praxisrelevanz von Lerninhalten in Projekten lässt sich zunächst als die Echtheit der Aufgabenstellung im Projekt verstehen. Darüber hinaus verbinden sich im Kontext Deutsch als Fremdsprache/Deutsch als Zweitsprache die Authentizität von Sprache, Orten und Situationen (vgl. Schart 2003a: 96) und die gesellschaftliche Praxisrelevanz zu einem Feld der Kritik daran, wie wenig und in welch geringem Ausmaß die Praxis des DaF/DaZ-Unterrichts in die zielsprachigen Gesellschaften hineinreicht. Authentizität bedeutet hier also das Hineinwirken der, Wirklichkeit' in Unterrichtsbzw. Lernsituationen, andererseits aber auch das Hinauswirken der Lernaktivitäten in die gesellschaftliche Wirklichkeit. Die Didaktik des Deutschen als Fremd- und Zweitsprache hat in besonderem Maße die Aufgabe Wege anzubieten, wie Lernende sprachlich und gesellschaftlich ernst zu nehmende Akteure in den Zielsprachengesellschaften sein können. Sie hat damit nicht zuletzt gesellschaftliche Partizipation zu ermöglichen. Fremdsprachenunterricht muss nicht nur dazu befähigen an zielsprachiger Kommunikation situationsadäquat teilnehmen zu können, sondern muss darüber weit hinausgehend Anlässe schaffen, in denen diese Kommuni- 
kation notwendig und für Lernende als Akteure von praktischer Relevanz wird (vgl. Peuschel 2007). Gerade Projekte aktiver Radioarbeit versuchen, die bewusste Auseinandersetzung mit der zu lernenden Sprache im Klassenzimmer mit der Echtheit, Authentizität und der Partizipation an zielsprachigen, öffentlichen medialen Diskursen zu vereinen.

\section{Aktive Radioarbeit in der Fremdsprache Deutsch}

In sprachspezifischen Projekten aktiver Medienarbeit werden verschiedene Stränge des Fremdsprachenlernens verbunden: gelebte Autonomie, Sprachverwendung durch aktive und produktive Mediennutzung und dadurch aktive Teilhabe an öffentlichen zielsprachigen Diskursen. Produktive Medienarbeit wird in der medienpädagogischen Literatur als die ,ziel-, adressaten- und beziehungsorientierte Nutzung von Audio-, Foto-, Druck-, Film-, Video und Computerhardware und Software zur Eigenproduktion von Kommunikaten in pädagogisch begründeter, projektorientierter Gruppenarbeit [...]“ definiert (Thiele 1999: 62). Interkulturelle medienpädagogische Projekte stoßen nicht selten auf sprachliche Barrieren, und beklagen nach der recht oberflächlichen Sichtung der Literatur der Nachbardisziplin DaF, dass dort „Medien meist ausschließlich zur Vermittlung von Unterrichtsinhalten verstanden [werden]“ (Maurer 2004: 61). Ebenso beklagen Medienpädagogen und Medienpädagoginnen, dass didaktische Überlegungen zum Umgang mit Sprachschwierigkeiten in der medienpädagogischen Literatur nicht gegeben werden. Auf der anderen Seite wiederum stellen beliebte Film- und Audioprojekte im Fremdsprachenunterricht nicht selten zu hohe Anforderungen an die technische und die Medienkompetenz von Sprachlehrkräften und Lernenden oder scheinen im zur Verfügung stehenden Zeitrahmen nicht machbar. Die Integration projektorientierten sprachlichen Lernens in den Unterricht, z.B. durch die Erstellung eines eigenen Hörspiels, scheint jedoch in beider Hinsicht, sprachlich und medial, zu sehr guten Ergebnissen zu führen (vgl. Wörfell 2002). Die Schnittpunkte zwischen beiden Disziplinen, DaF-Didaktik und Medienpädagogik, in der Konzeptionalisierung von Lernen als einer selbstverantwortlichen, handelnden und problemorientierten Tätigkeit liegen auf der Hand.

Aktive Medienarbeit im Fremdsprachenunterricht bedeutet eine Teilübertragung medienpädagogischer Konzepte auf das Fremdsprachenlernen unter den spezifischen Zielsetzungen des Fremdsprachenunterrichts. In aktiver Radioarbeit mit Deutschlernenden wird das Medium Radio bzw. podcast/Internet als auditives Medium für mündliche Produktionen genutzt. Alle Inhalte müssen über sprachliche oder zumindest auditive Signale vermittelt werden. Einleitungen ins Programm, Moderationen, Beiträge über ein selbst gewähltes Thema, Nacherzählungen von Liedtexten, Interviews, fiktive Hörstücke etc. sind typische (Radiosendungs)Formate, die hier als mündliche Lerneräußerungen in geplanten und strukturierten Produktionsprozessen entstehen. In Radioprojekten sind drei Aspekte 
relativ gleichberechtigt von Bedeutung - die inhaltliche Auseinandersetzung mit einem selbst gewählten Thema, die produktorientierte, medienspezifische, bewusste und absichtsvolle Modellierung der Fremdsprache und die technische Umsetzung durch wiederholte Sprachaufnahmen und deren Anordnung mit anderen Elementen zu sendefähigen Formaten. Diese verlassen den Rahmen des Klassenzimmers und werden Teil des öffentlichen medialen Diskurses, indem sie über UKW in v.a. lokalen Radiosendern ausgestrahlt, live gestreamt oder als podcast im Internet präsentiert werden. ${ }^{i}$ Das Medium Radio fordert fremd- oder mehrsprachige mündliche Produktionen und eröffnet Lernenden die Möglichkeit für und in einer realen, zielsprachigen Öffentlichkeit ihre Interessen und Meinungen zu äußern. Einzelaspekte der Entstehung und Entwicklung dieser mündlichen Äußerungen sind Gegenstand der hier vorgestellten Untersuchung.

\section{Sprachliches Lernen in Radioprojekten als integrierte Textentwicklung}

\subsection{Forschungsfragen und Untersuchungsdesign}

Die bereits angesprochene, notwendige empirische Exploration von Lernprojekten hinsichtlich ihres Potentials für das Lernen von Fremdsprachen fordert zunächst ein longitudinales Vorgehen, also die empirische Begleitung eines Radioprojekts in seinem Verlauf. Gleichzeitig erfordert dies die Konkretisierung von Fragestellungen auf Einzelaspekte im Projekt. Sprachliches Lernen in Projekten kann sich in zahlreichen sprachlichen Aktivitäten zeigen und in entsprechenden sprachlichen Produkten manifestieren, z.B. in der Interaktion zwischen den Lernenden bei der Gruppenarbeit im Projekt oder im Mailkontakt mit Muttersprachlern und Muttersprachlerinnen, in der Vorbereitung von Interviews für die Radiosendung etc. Ein Hauptinteresse der vorliegenden Studie liegt zunächst auf der Entstehung und Entwicklung der im Endprodukt „Radiosendung“ manifesten mündlichen Äußerungen der Lernenden. Obwohl in Radiosendungen medial Mündlichkeit präsentiert wird ${ }^{\mathrm{ii}}$, ist doch die Entwicklung der (mündlichen) Beiträge im Projekt von einem für das Fremdsprachenlernen typischen Neben- und Miteinander schriftlicher und mündlicher Produktionen geprägt. Dieses die produktiven Fertigkeiten integrierende Miteinander zu beschreiben und in Verbindung mit spezifischen sprachlichen Aktivitäten im Projekt zu interpretieren, ist Ziel der Studie. Folgende Forschungsfragen bilden die Basis für das Vorgehen:

1. Wie entwickeln sich mündliche und schriftliche Produktionen von Lernenden in medienspezifischen Projekten am Beispiel von Radioprojekten mit erwachsenen Deutsch-als-Fremdsprache-Lernenden?

2. Welche sprachlichen und außersprachlichen Aktivitäten tragen zur Entwicklung der Lernerproduktionen im Projekt bei? 


\subsection{Kontextualisierung der Studie - Radioprojekt im universitären}

Konversationskurs im Studiengang Dolmetschen/Übersetzen an der Karls-Universität Prag

Der Konversationskurs, in dessen Rahmen im WS 2005/2006 und im WS 2006/2007 je ein Radioprojekt durchgeführt wurde, ist fester Bestandteil des Studienangebots der Deutschabteilung der Karls-Universität Prag und wurde in den genannten Semestern von der jeweiligen DAAD- Sprachassistentin angeboten. Im Konversationskurs können studienrelevante Punkte erworben werden, der Kurs erstreckt sich über das gesamte Semester, d.h. von Mitte Oktober bis Anfang Februar, und findet einmal pro Woche jeweils 90 Minuten statt. Die Lehrkraft im WS 2005/2006 teilte den Kurs inhaltlich in zwei Teile. In den ersten ca. 45 Minuten hielten die Studierenden Referate und Vorträge zu selbst gewählten Themen vor der Seminargruppe. Im zweiten Teil (ca. 45 Minuten) widmete sich die Gruppe dem Radioprojekt, für das sich die Seminargruppe am Anfang des Semesters gemeinsam entschied. Die Projektidee stammte von der Lehrkraft. Es standen insgesamt 13 Termine für das Projekt zur Verfügung.

\begin{tabular}{|c|c|c|}
\hline Seminarwoche & $\begin{array}{l}\text { Projektphase } \\
\text { (nach Gudjons 1994: 84ff) }\end{array}$ & Allgemeine Inhalte \\
\hline 0 & Planung & Projektskizze der Lehrkraft \\
\hline $1-3$ & $\begin{array}{l}\text { Durchführung / Kooperative } \\
\text { Planung }\end{array}$ & Themenfindung \\
\hline $4-6 / 7$ & Durchführung & $\begin{array}{l}\text { Materialbeschaffung, Programmgestaltung, } \\
\text { Textproduktion }\end{array}$ \\
\hline $\begin{array}{l}7 \text { (+ Block- } \\
\text { seminar) }\end{array}$ & Durchführung & Testaufnahmen \\
\hline $8-9$ & Durchführung & $\begin{array}{l}\text { Aufnahmen, Textproduktion, Programmges- } \\
\text { taltung, Gestaltung des Beiheftes }\end{array}$ \\
\hline $10-11$ & [Ferien] & \\
\hline 12 & Durchführung & $\begin{array}{l}\text { Aufnahmen, Sendung zusammenstellen, } \\
\text { Schneiden, Projektprodukt (CD) fertig stel- } \\
\text { len }\end{array}$ \\
\hline 13 & Evaluation, Präsentation & Sendung, Abschlussdiskussion \\
\hline
\end{tabular}

Tab. 1: Projektverlauf „soundczech“.

Die Lernenden - Im WS 2005/2006 nehmen 14 Studierende am Konversationskurs teil. Die meisten Studierenden des Kurses absolvieren ihr erstes Semester an der Universität, einige bereits das dritte. Sie sind zwischen 18 und 20 Jahren alt, ken- 
nen sich (noch) nicht und haben unterschiedliche Deutschlernbiografien. Einige sind AbsolventInnen des deutschen Gymnasiums in Prag, einige haben sich bereits längere Zeit, z.T. bis zu einem Jahr, in der Bundesrepublik aufgehalten, andere wiederum haben das Nachbarland noch nie besucht. Bis auf zwei TeilnehmerInnen sprechen alle Tschechisch als Muttersprache. Eine Projektteilnehmerin ist eine Erasmusstudentin aus Spanien mit Spanisch als Muttersprache, eine andere Teilnehmerin ist deutsche Muttersprachlerin. Die Deutschkompetenzen der Studierenden variieren von selbständiger bis hin zu kompetenter Verwendung der Fremdsprache Deutsch. Systematische Sprachstandsdaten sind im Rahmen dieser Untersuchung nicht erhoben worden, dennoch lassen Details aus den Lernerinterviews interessante Einblicke in die individuellen Lernbiografien und sprachlichen Stärken und Schwächen zu.

Die Projektleiterin - Leiterin des Konversationskurses und damit Leiterin des Radioprojekts ist die an die Deutschabteilung der Karlsuniversität entsandte DAAD-Sprachassistentin, die hier ihr erstes Projekt mit Deutschlernenden durchführt. Als universitär ausgebildete $\mathrm{DaFlerin}$ verfügt sie über entsprechend fundierte Fachkenntnisse, hatte jedoch bis zum Zeitpunkt der Sprachassistenz noch keine Erfahrung mit der Durchführung von fremdsprachigen Lernprojekten. Sie trat in der Phase der Vorbereitung des Projekts an mich in meiner Rolle als „Expertin“ für Radioprojekte mit Deutschlernenden heran, um einige Hinweise zur Durchführung des Projekts zu erhalten.

Die Untersuchungsleiterin - Durch diese Kontaktaufnahme eröffnete sich für mich die Möglichkeit, nach einer Reihe von Vorstudien, die Untersuchung durchzuführen und nicht zugleich in der Rolle der Projektleiterin zu stehen. Dennoch hatte ich im Rahmen des Projekts eine zusätzliche Rolle, und zwar die der Kontaktperson zum Radiosender, dem lokalen, freien Radio der Stadt Leipzig, Radio Blau, d.h. ich war für das Senden des Produkts in der zielsprachigen Öffentlichkeit verantwortlich, wurde im Projekt von den Lernenden als native speaker und als Expertin in Sachen Radio wahrgenommen, an die sowohl technische als auch inhaltliche Fragen gerichtet werden konnten.

\subsection{Datenerhebung, -aufbereitung und -analyse}

Die Erhebung der Daten umfasst einerseits die Sammlung der schriftlich und/oder mündlich manifesten (Teil)produktionen der Lernenden im Projekt, d.h. Notizzettel, Probeaufnahmen, schriftliche Vorlagen für eingelesene Texte, Übersetzungsversuche etc., anderseits Interviews zu zwei Zeitpunkten des Projektes (Projektwoche 7, Projektwoche 12), die die eigenen sprachlichen Aktivitäten der Lernenden fokussierten. Als flankierende Daten wurden Interviews mit der Projektleiterin (Lehrkraft) durchgeführt und ebenso zwei Gruppenarbeitsphasen als Audiomitschnitt dokumentiert. 


\begin{tabular}{|l|l|}
\hline Mündlich & Schriftlich \\
\hline Tests und Probeaufnahmen & Notizen, Stichpunkte, Textentwürfe \\
\hline $\begin{array}{l}\text { Endaufnahmen für das Projektprodukt (die } \\
\text { Radiosendung “soundczech - Im Osten viel } \\
\text { Neues“) }\end{array}$ & $\begin{array}{l}\text { Textvorlage für die Aufnahmen für das Endpro- } \\
\text { dukt ,Radiosendung“ }\end{array}$ \\
\cline { 1 - 2 } $\begin{array}{l}\text { Äußerungen zur inhaltlichen Vorbereitung des } \\
\text { Projektprodukts (Umfrage unter deutschen } \\
\text { Touristen in Prag) }\end{array}$ & $\begin{array}{l}\text { E-Mails zur inhaltlichen Vorbereitung des Pro- } \\
\text { jektprodukts (z.B. an einen deutschen Liederma- } \\
\text { cher) }\end{array}$ \\
\cline { 1 - 2 } Äußerungen im Plenum in der Seminargruppe & $\begin{array}{l}\text { Übersetzungsversuche und Übersetzungen von } \\
\text { Liedtexten }\end{array}$ \\
\cline { 1 - 2 } Äußerungen in der Arbeitsgruppe & \multicolumn{2}{|l}{$\begin{array}{l}\text { (Äußerungen im Rahmen der retrospektiven } \\
\text { Interviews) }\end{array}$} &
\end{tabular}

Tab. 2: Übersicht Projektdaten „soundczech“.

Die mündlichen Äußerungen wurden mit einem digitalen Audioaufnahmegerät aufgenommen (I-river). Die mündlichen Test- und Probeaufnahmen stammen aus dem Blockseminar in Projektwoche 7, die Endprodukte aus Projektwoche 9 und 12. Die im gesamten Projektverlauf entstandenen schriftlichen Produktionen der Lernenden wurden in Projektwoche 12 eingesammelt.

Die Art und Ausführlichkeit der Aufbereitung und Transkription der Rohdaten orientiert sich an deren weiterer Verwendung in der Analyse und an den Zielstellungen der Untersuchung (vgl. Kuckartz 2007). Da an die einzelnen Daten unterschiedliche Ziele gestellt sind, unterscheidet sich auch die Art der Transkription. Die monologischen mündlichen Äußerungen der Lernenden werden zunächst in normales Schriftdeutsch übertragen, wobei Pausen mit einer Länge $(>=0,28 \mathrm{~s})$ in Klammern in den Fließtext eingetragen werden. Doppelungen von Einzelwörtern, Neuanfänge, Abbrüche usw. werden transkribiert, Intonationsmuster zunächst nicht. ${ }^{\text {iii }}$

MAJA - Zwischenprodukt_mündlich_Text 3

Aufnahmedatum: Projektwoche 7

Aufnahmeort: Studentenwohnheim, Laptop

Länge des Beitrags gesamt: 13,1 Sekunden

Art des Textes: Probeaufnahme für den Beitrag über die tschechische Band Vypsana Fixa

00/ Ok und das was jetzt spielt ist die Vypsana Fixa die ausgeschriebene Filzstift

$01 /(0,9)$ die den Lied $(0,6)$ die Taucher $(0,4)$ sinkt singt (oh Mann ich kann nicht 02/ Deutsch)

Abb. 1: Beispieltranskript mündliches Projektprodukt. 
Die Originale der schriftlichen Lernendenproduktionen, also der Notizen, Textversionen und Textvorlagen wurden zunächst gescannt und in einem zweiten Schritt in ein herkömmliches Textverarbeitungsformat übertragen.

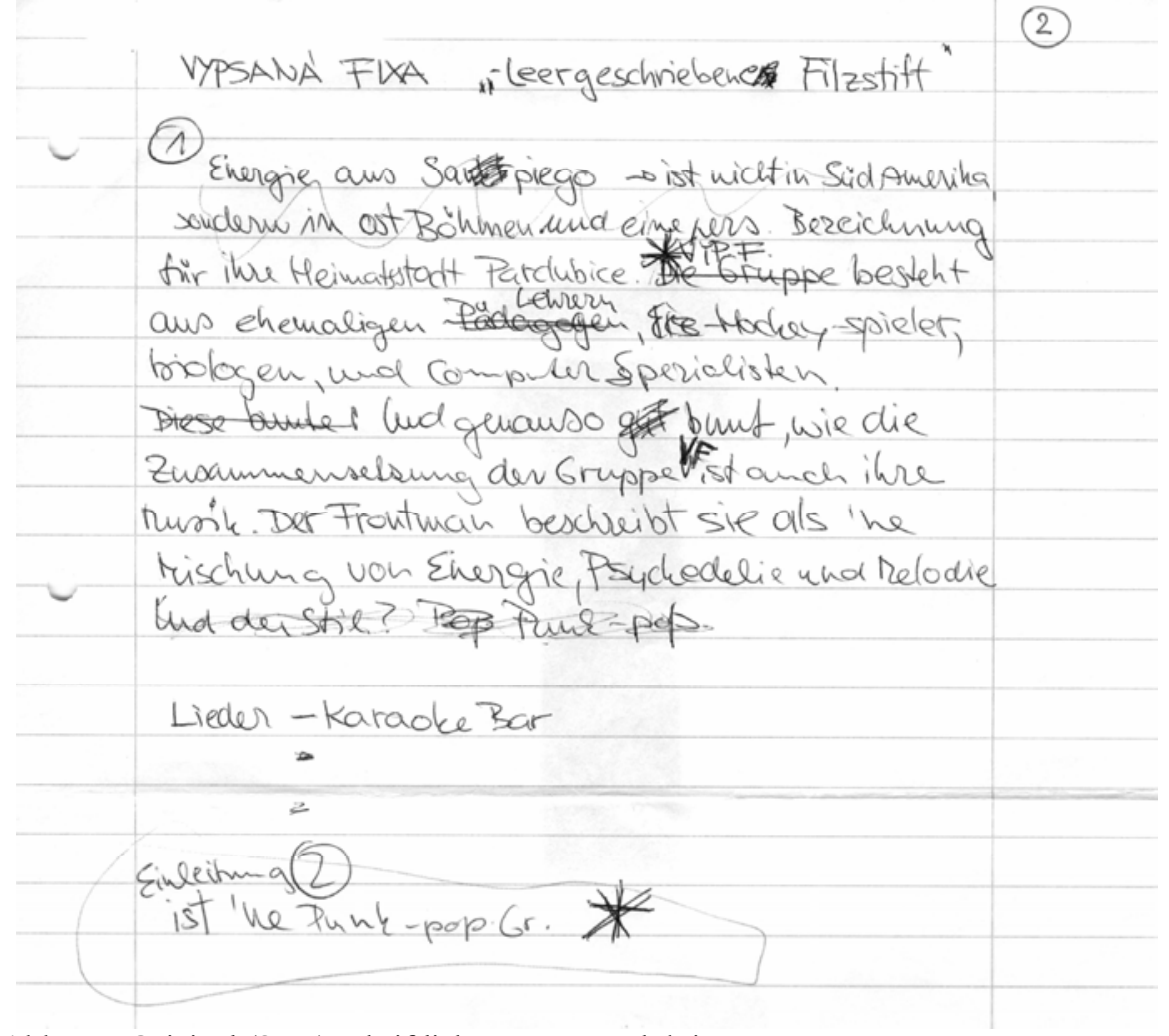

Abb. 2 - Original (Scan) schriftliche Lernerproduktion.

Für die digitalen Abschriften schriftlicher Lernendenproduktionen in ein Textverarbeitungsprogramm wurden folgende Regeln festgelegt, die jedoch mit fortschreitender Analyse der Texte weiter ausdifferenziert werden müssen. In die zeilenweise Übertragung der schriftlichen Texte wurden zunächst folgende Revisionen aufgenommen. ${ }^{\text {iv }}$

\begin{tabular}{|l|l|}
\hline Hochgestellt & $\begin{array}{l}\text { Addition, d.h. eigene Zusätze und Nachträge der Text- } \\
\text { autorInnen }\end{array}$ \\
\hline Sinfach durchgestrichen & Substitution, d.h. reparierte Passage \\
\hline Grau unterlegt & Deletion, d.h. gestrichene Passage \\
\hline & $\begin{array}{l}\text { Korrekturen und Änderungen von Mitstudierenden } \\
\text { (KoautorInnen) }\end{array}$ \\
\hline
\end{tabular}

Tab. 3: Legende: Übertragungskonvention schriftliche Lernerproduktion. 
Das Original (Abb. 2) stellt sich nach der Übertragung wie folgt dar:

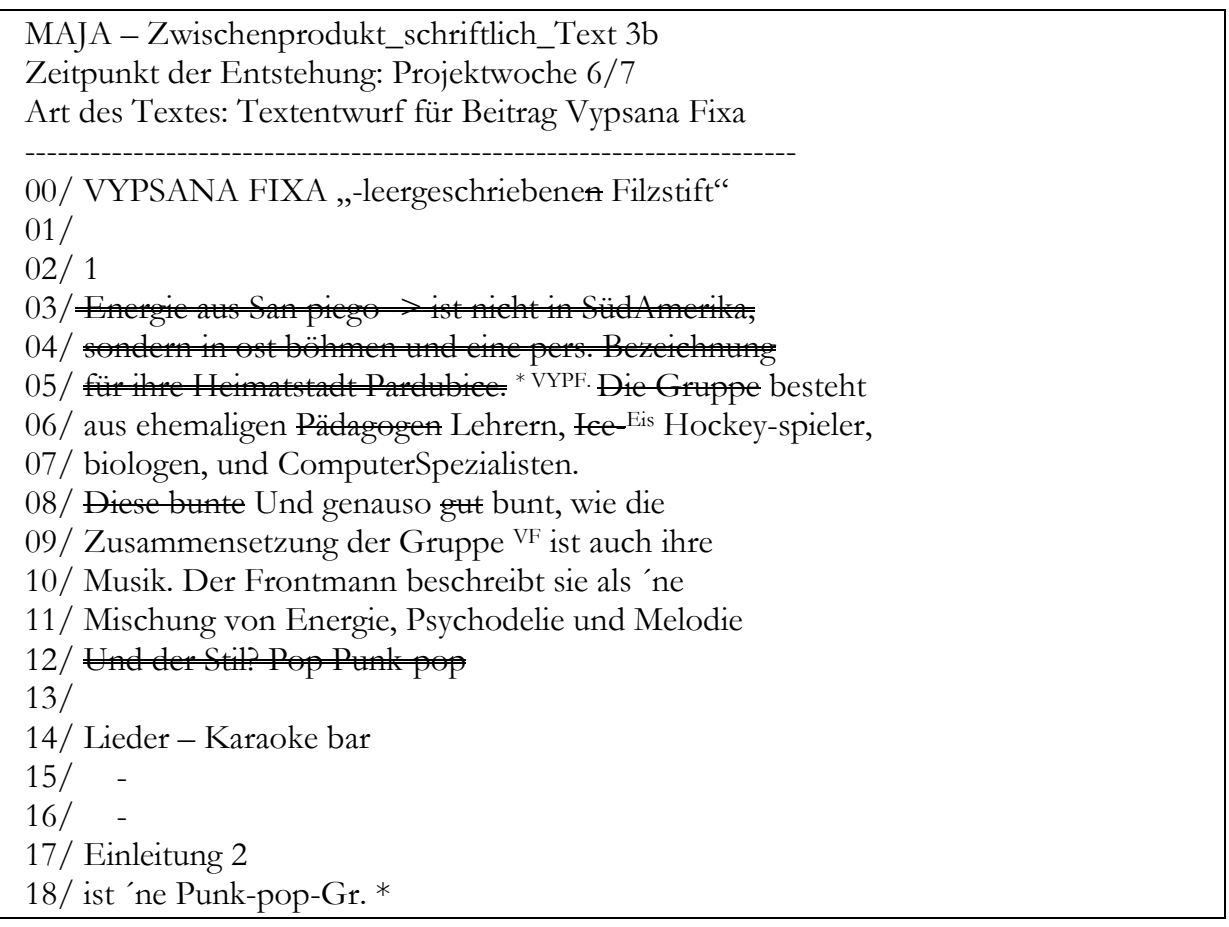

Abb. 3: Übertragungsresultat schriftliche Lernerproduktion - MAJA Textentwurf für Beitrag „Vypsana Fixa“" (Text 3b).

Ziel der nun einsetzenden Analyse der projektproduktbezogenen mündlichen und schriftlichen Produktionen ist es, Textentwicklungsprozesse nachvollziehbar zu machen und diese detailliert zu beschreiben. Die mündlichen Produktionen werden zunächst hinsichtlich ihrer Flüssigkeit untersucht (vgl. Stevener 2003), die schriftlichen Produktionen hinsichtlich der Entwicklung ihrer inhaltlichen Komplexität, morphosyntaktischen Korrektheit und ihrer Funktionalität. Dafür werden textuell manifeste Revisionen (vgl. Wrobel 1995) in verschiedenen Textversionen bis zum Endprodukt untersucht und zu den Kriterien Komplexität, Korrektheit und Funktionalität der Texte in Beziehung gesetzt. Die Arbeit an den sprachlichen Daten ist derzeit noch als zyklisch angelegte work in progress zu verstehen und soll im folgenden Kapitel am Beispiel von MAJAs Textproduktionen erläutert werden. 


\section{$5 \quad$ Interessante Zwischenergebnisse - Fertigkeiten übergreifende Textproduktion in Radioprojekten}

Ausgehend vom mündlichen Endprodukt, dem hier leider nur in der Transkription (vgl. Abb. 4) zugänglichen Radiobeitrag über die tschechische Punkpop-Band Vypsana Fixa, und der Betrachtung der Textoberfläche des Beitrags, fällt dessen große Korrektheit und stilistische Ausarbeitung sofort ins Auge. Ein einziger Versprecher, den die Sprecherin selbst repariert (Zeile 06-07: surrealischtisch surrealistischen) und ein einziger Neuanfang nach einer etwas längeren Pause (Zeile 07: ver $(0,95)$ verschieden) lassen auf eine hohe Sprechflüssigkeit schließen, ebenso wie die durchschnittlich kurze Dauer der Sprechpausen, die alle unter einer Sekunde bleiben. Eine phonetische Transkription würde vereinzelt Abweichungen von der standarddeutschen Aussprache aufzeigen, die zum großen Teil auf Interferenzen aus der Muttersprache Tschechisch zurückgeführt werden können und typische Ausspracheschwierigkeiten betreffen, wie z.B. die Artikulation von /e/-Lauten, Wortakzentverschiebungen und Vokallängenunterschiede.

MAJA - ENDPRODUKT_mündlich_Text 3

Aufnahmedatum: Projektwoche 9

Aufnahmeort: Phonetiklabor, Karlsuniversität Prag

Länge des Beitrags gesamt: 54 Sekunden

Art des Textes: Beitrag über die tschechische Band „Vypsana Fixa“

01/ Habt ihr schon mal einen leergeschriebenen Filzstift singen gehört $(0,49)$ das ist 02/ nämlich die genaue Übersetzung des Namen der ostböhmischen Punkpopband

03/ Vypsana Fixa (0,79) Vypsana Fixa besteht aus ehemaligen Lehrern $(0,29)$

04/ Eishockeyspielern Biologen und Computerspezialisten $(0,59)$ und genauso bunt

$05 /$ wie die Zusammensetzung der Band $(0,4)$ ist auch ihr Musikstil $(0,7)$ der Frontman

06/ beschreibt sie als eine Mischung aus Psychodelie und Energie gekoppelt mit

07/ Melodie $(0,7)$ die Jungs sind also keineswegs schlaffe Filzstifte ohne Saft $(0,53)$

$08 /$ und wovon sie singen $(0,4)$ eigentlich vom Leben [doch] die surrealischtisch

$09 /$ surrealistischen Texte lassen sich ver $(0,95)$ verschieden interpretieren $(0,52)$

$10 /$ zuweilen könnte man sie als ein wenig $(0,51)$ sinnentfremdet bezeichnen $(0,49)$

$11 /$ der Band geht es eben nicht um intellektuelle Ergüsse $(0,27)$ sondern hauptsächlich 12/ um Spaß

Abb. 4: MAJA Transkript Beitrag über die tschechische Band „Vypsana Fixa Endprodukt“ (Text 3).

Aufschlussreich für den Nachvollzug des innerhalb mehrerer Wochen verlaufenden Entwicklungsprozesses des Textes sind die dokumentierten Zwischenprodukte, die zeigen, wie aus einem Notizzettel eine Vorlage für den gelesenen Beitrag im Rahmen der Radiosendung „Soundczech“ entsteht. Das folgende Beispiel zeigt ein schriftliches Anfangsstadium des Textes in Projektwoche 4 und/oder 5 (Abb.5). Bis zu diesem Zeitpunkt hatten sich die Studierenden in Arbeitsgruppen aufgeteilt, 
die sich je einem spezifischen musikalischen Stil und damit einzelnen Bands und Künstlerinnen widmen, um diese in der Sendung vorzustellen. In der insgesamt sehr aktiven Arbeitsgruppe von MAJA waren drei weitere Studentinnen, wobei MAJA mit einer vergleichsweise häufigen Präsenz als Sprecherin in der Radiosendung eine sehr aktive Rolle innehat.

Das Transkript des Stichpunktzettels von MAJA (Abb. 5) weist die parallele Verwendung von Deutsch und Tschechisch auf (Zeile 08: Koncert; Zeile 10: Musikfestivala) und relativ häufig grammatische Normabweichungen (Zeile 09: Texten in Tschechisch; Zeile 11: Einer der beliebsten Gruppen). Nur sehr wenige der im Endprodukt vorhandenen Inhalte waren bereits hier angedacht und niedergeschrieben (Zeile 04: Namen der Band; Zeile 08: Mischung von Energie, Psychodelie, Melodie; Zeile 10: Sty/il -> Punk-pop).

MAJA - Zwischenprodukt_schriftlich_Text 3a

Zeitpunkt der Entstehung: Projektwoche 4/5

Art des Textes: Stichpunktzettel

$01 /$

$02 /$

03/ FIXA

04/ Namen der Band

$05 /$

$06 /$

$07 /$

08/ 1. Koncert 1994

$09 /$

10/ Musikfestivala

11/ Einer der beliebsten Gruppen XX jungen/r Leute

$12 / \mathrm{CDS}-7 \mathrm{E}$

\section{Karaoke bar}

schnell - Energie

spielen auch in Dt., Holland, Polen, Italien

Mardis Charakeristik:

Mischung von Energie, Psychodelie, Melodie. Texten in Tschechisch.

Sty/il -> Punk-pop

Abb. 5: MAJA Stichpunktzettel (Text 3a).

Zahlreiche Ideen und Propositionen sind nicht in das Endprodukt eingegangen, so z.B. Angaben zum Preis einer CD (Zeile 12: CDS - 7 E), zum ersten Konzert, den Spielorten oder Informationen zu Festivals oder den tschechischen Texten (Zeilen 04, 08, 09). Allerdings wird letzteres beispielsweise in einem Interview aufgegriffen, dass MAJA und eine Mitstudentin mit einem Mitglied der Band Vypsana Fixa für die Radiosendung führen, übersetzen und medial aufbereiten.

In der weiteren Entwicklung des Textes (vgl. Abb. 3, Text 3b) wird die Übersetzung des Namens der Band hinzugefügt (Zeile 00: VYPSANA FIXA „,leergeschriebenen Filzstift"), die Mitglieder der Band werden beschrieben (Zeile 05 - 07: * VYPF. Die Gruppe besteht aus ehemaligen Pädagegen Lehrern, Ice-Eis Hockey-spieler, biologen, und ComputerSpezialisten.), eine Formulierung über die Herkunft der Band wird verworfen (Zeile 03 - 05: $\Longrightarrow$ nicht in Süd merita, sonden in böhmen und eine pers. Bezehnung für ihre 
Eine hier entwickelte Formulierung wird bis zur Endversion nicht weiter geändert (Zeile 10 - 11: Der Frontmann beschreibt sie als 'ne Mischung von Energie, Psychodelie und Melodie) und beinhaltet den Versuch, mündlichsprachige Elemente ('ne) einzubringen. Die Information über den Stil der Band wird als Frage formuliert aber nicht zu Ende geführt und wieder verworfen (Zeile 12: Sußerdem sind bereits die Struktur und Funktion des Textes innerhalb der gesamten Sendung ersichtlich (Zeile 02: 1; Zeile 17: Einleitung 2). Deutlich erkennbar ist die inhaltliche Erweiterung des Textes durch neue Informationen, durch ausformulierte Sätze und durch eine stärkere Strukturierung des Textes.

Während bis hierhin alle Revisionen von der Autorin selbst vorgenommen wurden, weist die anschließende Textversion (Abb. 6, Text 3c) außerdem Korrekturen und Änderungen einer Mitstudentin auf.

MAJA - Zwischenprodukt_schriftlich_Text 3c

Zeitpunkt der Entstehung: Projektwoche 7

Art des Textes: Textentwurf für Beitrag „Vypsana Fixa“

00/ Haben Sie schon mal 'ne leerg. F. 'nen leergeschriebenen Filzstift `signgen gehört?

01/ Das ist nämlich die genaue Übersetzung des Namen

02/ der östböhmischen Punk-Pop-Gruppe. - VYP.F.

02/ VF besteht aus ehemaligen Lehrern, Eis-hockey Spieler,

04/ Biologen und Comp-Spezialisten.

05/ Und genauso bunt, wie die Zusammensetzung der

06/ Band-Ge VF, ist auch ihre Musik. Musikstil / die Stilbreite ihrer Musik

07/ Der Frontman beschreibt sie als eine Mischung -aus

08/ Psychedilly u. Energie gekoppelt mit Melodie

09/ Die Jungs sind also keineswegs schlaffe Filzstifte ohne Saft.

10/

11/ und wozu wovon sie singen? Eigentlich vom Leben,

12/ aber ihre surrealistischen Texte kann man auch als

13 etwas sinnentfremdet bezeichnen. Kurz gesagt,

Abb. 6: MAJA Textentwurf für Beitrag „Vypsana Fixa“ (Text 3c).

In dieser Folgeversion werden als wichtige Neuerung die zukünftigen Adressaten des Textes, d.h. die Hörerschaft der Sendung, explizit angesprochen (Zeile 00: Haben Sie schon mal 'ne leerg. F. ‘signgen gehört?) und damit die Funktion des Textes spezifiziert. Die Übersetzung des Namens der Band ist in Satzform gebracht und ausformuliert (Zeile 00 - 01). Eine erneute inhaltliche Erweiterung erfährt der Text durch die Informationen zum Inhalt der Lieder (Zeile 10 - 13: [...] als den vorhergehenden Textversionen sind jedoch vor allem zwei Dinge: der Versuch einer inszenierten Mündlichkeit (Apostrophierungen, „nämlich“, kurze Sätze) und 
die zahlreichen Änderungsvorschläge der zweisprachigen Mitstudentin, die als Koautorin dem Text eine stärker umgangssprachliche Komponente gibt.

Die vierte und letzte geschriebene Version des Textes (Abb. 7, Text 3d) dient als Vorlage für den Beitrag, der in der Radiosendung als Endprodukt zu hören ist und wurde von der Mitstudentin niedergeschrieben. Es sind nur noch wenige Revisionen (Zeile 01: Habt Ihr; Zeile 07: S: erkennbar und der Text weist mit Leerzeilen und Satzanfängen auf je einer neuen Zeile eine (vor)lesefreundliche Struktur auf.

MAJA - Zwischenprodukt_schriftlich_Text 3d

Zeitpunkt der Entstehung: Projektwoche 4 - 7

Art des Textes: Textvorlage für Aufnahme für Sendung

00/ Vyspana Fixa

01/ Habt Ihr (Haben Sie) schon mal 'nen leergeschriebenen Filzstift singen gehört?

02/ Das ist nämlich die genaue Übersetzung des Namen der ostböhmischen

03/ Punk-Pop Band VYP.F.

04/ VYP.F. besteht aus ehemaligen Lehrern, Eishockeyspielern, Biologen und

05/ Computerspezialisten.

06/ Und genauso bunt wie die Zusammensetzung der Band ist auch

07/ihr Musikstil. S S

08/ Der Frontman beschreibt sie als eine Mischung aus Psychidilly

09/ u. Energie gekoppelt mit Melodie.

10/ Die Jungs sind also keineswegs schlaffe Filzstifte ohne Saft.

11/ Und wovon sie singen? Eigentlich vom Leben, doch die

12/ surrealistischen Texte lassen sich verschieden interpretieren, zuweilen

13/ könnte man sie als ein wenig sinnentfremdet bezeichnen.

14/ Der Band geht es eben nicht um intellektuelle Ergüsse, sondern

15/ hauptsächlich um Spaß

Abb. 7: MAJA Textvorlage für Aufnahme für Sendung (Text 3d).

Die Texte 3a bis 3d machen die Entwicklung der geschriebenen Vorlage eines Textes nachvollziehbar, der Teil einer Radiosendung sein soll, d.h. der als Vorlage für eine gelesene mündliche Produktion dient. Die Adressaten des Textes sind nicht die Lehrkraft oder Mitstudierende, sondern deutsche Radiohörerinnen und hörer. Neben den schriftlichen Versionen gehören zur Entwicklung des Endprodukts ebenso die mündlichen Zwischenprodukte, die MAJA im Verlauf des Projekts produziert hat. Abb. 8 zeigt noch einmal die dokumentierte Probeaufnahme von MAJA die in einer lockeren Umgebung eher spielerisch entstanden ist. 
MAJA - Zwischenprodukt_mündlich_Text 3

Aufnahmedatum: Projektwoche 7

Aufnahmeort: Studentenwohnheim, Laptop

Länge des Beitrags gesamt: 13,1 Sekunden

Art des Textes: Probeaufnahme für den Beitrag über die tschechische Band Vypsana Fixa

00/ Ok und das was jetzt spielt ist die Vypsana Fixa die ausgeschriebene Filzstift

$01 /(0,9)$ die den Lied $(0,6)$ die Taucher $(0,4)$ sinkt singt (o Mann ich kann nicht

02/ Deutsch)

Abb. 8: MAJA Probeaufnahme Beitrag Vypsana Fixa

Im Vergleich zur mündlichen Produktion des Endprodukts (vgl. Abb. 4) können morphosyntaktische Unkorrektheiten identifiziert werden (die ausgeschriebene Filzstift/den Lied) und Ausspracheschwierigkeiten beim / ng/-Laut, die zu einer phonetischen Selbstreparatur führen (Zeile 01: sinkt singt). Vergleicht man die Sprechrate und die Artikulationsratev der beiden mündlichen Äußerungen, ist neben der offensichtlichen Länge des Betrags eine Vergrößerung beider Parameter festzustellen (Tab. 4).

\begin{tabular}{|l|l|l|}
\hline & Mündliches Zwischenprodukt & Mündliches Endprodukt \\
\hline Gesamtzeit & $13,1 \mathrm{~s}$ & $53,6 \mathrm{~s}$ \\
\hline Artikulierte Zeit & $11,2 \mathrm{~s}$ & $46,0 \mathrm{~s}$ \\
\hline $\begin{array}{l}\text { Anzahl der Silben } \\
\text { (gesamt) }\end{array}$ & 35 & 218 \\
\hline Anzahl der Pausen & 3 & 14 \\
\hline $\begin{array}{l}\text { Nicht phonierte Zeit } \\
\text { (gesamt) }\end{array}$ & $1,9 \mathrm{~s}$ & $\mathbf{7 , 6} \mathrm{s}$ \\
\hline Sprechrate & $\mathbf{1 6 0 , 2}$ Silben pro Minute & $\mathbf{2 4 3 , 8}$ Silben pro Minute \\
\hline Artikulationsrate & $\mathbf{1 8 7 , 5}$ Silben pro Minute & $\mathbf{2 8 4 , 3}$ Silben pro Minute \\
\hline
\end{tabular}

Tab. 4: Vergleich Flüssigkeit MAJA Probeaufnahme und MAJA Endprodukt.

Beide mündlichen Texte unterscheiden sich außerdem in der Art der mündlichen Produktion, im Grad ihrer Elaboration, in der Intensität ihrer Vorbereitung und damit im Grad der Spontaneität der Äußerung zwischen freier mündlicher Äußerung und (vor)gelesener mündlicher Äußerung. 
Anhand des Beispiels MAJA und der Beschreibung der Entwicklung eines Einzeltextes im Mündlichen wie im Schriftlichen ist dargestellt worden, wie qualitativ hochwertige, adressatenspezifische und an den Interessen der Lernenden orientierte sprachliche Projektprodukte in einem Radioprojekt entstehen. Werden weitere Beispiele von Textentwicklungsprozessen in Radioprojekten betrachtet, wird sich vermutlich große individuelle Varianz zeigen. Dennoch scheint das Nachvollziehen integrierter, also die Grenzen der einzelnen Fertigkeiten überschreitenden Textentwicklungsprozesse Anhaltspunkte zu bieten, sprachliches Lernen in Projekten empirisch zu fassen.

Textproduktionsprozesse in (Radio)Projekten sind an einzelne Projektphasen und Projektschritte gebunden. Der Formulierung eigener Ideen in der Fremdsprache (Abb. 3, Text 3b) geht die Ideen- und Themenfindung im Projekt und in der Arbeitsgruppe voraus (Abb. 5, Text 3a). Gruppenarbeitsprozesse und Ergebnisse von inhaltsorientierten Recherchen manifestieren sich in einer größeren Informationsdichte und längeren, kooperativ revidierten Textentwürfen (Abb. 6, Text 3c), die dann schließlich, entsprechend dem Projektziel in eine vorlesefreundliche und hochgradig zielsprachennahe Version gebracht, als mündliches Endprodukt produziert werden (Abb. 7, Text 3d und Abb. 4, Text 3).

\section{Schlussfolgerungen}

Es ist sicher verfrüht, lernfördernde Aktivitäten in Radioprojekten zu benennen, bevor nicht weitere Beispiele integrierter Textentwicklung empirisch untersucht werden. Es kann jedoch gezeigt werden, dass strukturierte fremdsprachige Radioprojekte die Produktion von qualitativ hochwertigen fremdsprachigen Texten ermöglichen und sich die Lernenden dabei nicht nur mit einem selbst gewählten Thema auseinandersetzen, sondern auch mit dessen Präsentation in schriftlicher und mündlicher Form für die zielsprachige Hörendenschaft.

Sprachliches Lernen in Radioprojekten umfasst ausgeprägte mündliche und schriftliche Komponenten, die integrierend und als Teil der Aufgabe, ein auditives Projektprodukt zu erstellen, erforderlich sind. Auf dem Weg zum fertigen Radiobeitrag werden nicht nur zahlreiche sich textuell manifestierende sprachliche Aktivitäten von den Lernenden vorgenommen, sondern auch solche, die sich nur indirekt in den Texten nachweisen lassen, wie z.B. das Recherchieren von relevanten Informationen, deren adressatenspezifische Auswahl, das Anfertigen von Übersetzungen, das Einüben des Sprechens bzw. Lesens der Texte vor der endgültigen Aufnahme etc. Im weiteren Verlauf der Untersuchung ist es nötig, ein Produktionsmodell zu entwickeln, dass die produktiven Fertigkeiten integriert betrachtet und die äußeren Bedingungen der Textentwicklung gleichberechtigt zu den textinternen Entwicklungen fassen kann. Ein erweitertes Modell des Formulierens (vgl. Wrobel 1995) bietet hierfür interessante Anhaltspunkte: 
Formulieren $[\ldots]$ ist als revidierendes Formulieren selbst ein textgenerativer Prozeß, in dem Texte auf der Basis konzeptuellen, pragmatischen und sprachlichen Wissens entwickelt werden. Gerade die Inkongruenzen zwischen diesen verschiedenartigen Wissensdomänen sind es, die den Formulierungsprozeß $[\ldots]$ prägen. Formulieren ist deshalb weniger Übersetzung als vielmehr Adaption: ein Prozeß der wechselseitigen Anpassung konzeptuellen Wissens, kontextueller und kotextueller Erfordernisse und sprachlicher Strukturformen, in dem ein Text als sinnvolle Einheit dessen entsteht, was der Schreiber mit ihm meint, sagt und tut (Wrobel 1995: 124f).

Die wechselseitige Anpassung unterschiedlicher Wissensdömänen kann und sollte nicht zuletzt auch zu sprachlichen Lernprozessen führen. Lernen in Sprachlernprojekten ist sprachliches Lernen, dass lernendenorientiert auf der Grundlage einer echten Aktivität, handlungsorientiert und themenspezifisch die Auseinandersetzung mit der Zielsprache und deren aktive Verwendung fördert. Weitere Untersuchungen zum sprachlichen Lernen in Projekten sind jedoch notwendig, um detaillierte Aussagen über mögliche und tatsächliche Lernfortschritte in der LehrLernform (Radio)Projekt treffen zu können.

\section{Literatur}

Bausch, Karl-Richard; Burwitz-Melzer, Eva; Königs, Frank G. \& Krumm, Hans-Jürgen (Hrsg.) (2006): Aufgabenorientierung als Aufgabe. Tübingen: Narr.

Dietrich, Ingrid (1995): Übungen und Arbeitsformen im Projektunterricht. In: Bausch, Karl-Richard; Christ, Herbert \& Krumm, Hans-Jürgen (Hrsg.) (1995): Handbuch Fremdsprachenunterricht. Tübingen Francke, 255-258.

Edwards, Jane A. \& Lampert, Martin D. (1993): Talking Data. Transcription and Coding in Discourse Research. Lawrence Erlbaum Associates: New Jersey.

Fiehler, Reinhard; Barden, Birgit; Elstermann, Mechthild \& Kraft, Barbara (2004): Eigenschaften gesprochener Sprache. Tübingen: Narr.

Funk, Hermann (2006): Aufgabenorientierung in Lehrwerk und Unterricht - das Problem der Theorie mit der Vielfalt der Praxis. In: Bausch, Karl-Richard et al. (Hrsg.), 52-61.

Gudjons, Herbert (1994): Handlungsorientiert lehren und lernen: Scbüleraktivierung - Selbständigkeit - Projektarbeit. Bad Heilbrunn: Klinkhardt.

Henrici, Gert; Königs, Frank G. \& Zöfgen, Eckehard (Hrsg.) (2003): Mündliche Produktion in der Fremdsprache (Fremdsprachen Lebren und Lernen, 32).

Jahn, Susanne \& Schier, Carmen (2004): Seminaristisches Arbeiten im Rahmen des Sprachund Landeskundeunterrichts in Portugal. In: InfoDaF 29, 344-355.

Kuckartz, Udo (2007): Einführung in die computergestütz̧te Analyse qualitativer Daten. Wiesbaden: VS Verlag. 
Legutke, Michael (2006): Aufgabe - Projekt - Szenario. In: Bausch, Karl-Richard et al. (Hrsg.), 140-148.

Maurer, Björn (2004): Medienarbeit mit Kindern aus Migrationskontexten. München: kopaed.

Mayring, Phillip (2002): Einführung in die qualitative Sozialforschung. Eine Anleitung zum qualitativen Denken. Weinheim: Belz.

Müller-Hartmann, Andreas \& Schocker-von Ditfurth, Marita (2004): Projektorientiertes Lernen mit neuen Medien in der fremdsprachlichen Lehrerbildung. In: Barkowski, Hans \& Funk, Hermann (Hrsg.): Lernerautonomie und Fremdsprachenunterricht. Berlin: Cornelsen, 89-107.

Peuschel, Kristina (2007): Kommunikation und Teilhabe - Wie Deutschlernende in Radiound podcast-Projekten zu medial präsenten Sprachnutzenden werden. In: Deutsch als Zweitsprache 2, 33-41.

Piepho, Hans-Eberhard (2003): Von der Übungs- und Aufgabentypologie zur Szenariendidaktik - es hat sich was entwickelt. In: Legutke, Michael \& Schocker-von Ditfurth, Marita (Hrsg.) (2003): Kommunikativer Fremdsprachenunterricht: Rückblick nach vorn (Festschrift für Christoph Edelhoff). Tübingen: Narr, 59-68.

Raasch, Albert (1997): Lernen von Sprache: Diskussion und Perspektiven. In: MüllerVerweyen, Michael (Hrsg.): Neues Lernen - Selbstgesteuert - Autonom. München : GoetheInstitut, 9-32 [Standpunkte zur Sprach- und Kulturvermittlung, 7].

Reuken, Dagmar \& Hentschel, Uwe (1991): Wege zu freierem Sprechen: Erfahrungsbericht über ein Training zur Reduktion von Sprechängsten bei ausländischen Studierenden. In: Materialien DaF 34, 237-265.

Schart, Michael (2003a): Projektunterricht - subjektiv betrachtet. Eine qualitative Studie mit Lebrenden für Deutsch als Fremdsprache. Hohengehren: Schneider.

Schart, Michael (2003b): Was ist das eigentlich: Projektunterricht - Ein fiktives Gespräch über eine vage Idee. In: Info DaF 30(6), 576-593.

Schwarzmeier, Antje \& Werner, Ulrike (2007): Interaudio - Materialien für die interkulturelle Radioausbildung. Kassel: Hessische Landesanstalt für privaten Rundfunk.

Šimunek, Robert (2007): Formelhafte Sprache und Internetprojekte - Zum Sprachgebrauch und Spracherwerb fortgeschrittener Fremdsprachenlerner. Tübingen: Narr.

Stevener, Jan (2003): Aufmerksamkeit, Automatisierung und Monitoring. Zur Forschungsmethodik. In: Fremdsprachen Lehren und Lernen 32, 27-49-

Thiele, Günter A. (1999): Produktive Medienarbeit in der Schule - heute. In: Baacke, Dieter; Kornblum, Susanne; Lauffer, Jürgen, Mikos, Lothar \& Thiele, Günter A. (Hrsg.) (1999): Handbuch Medien: Medienkompetenz - Modelle und Projekte. Bonn: Bundeszentrale für politische Bildung.

Wörfell, Birgit (2002): Projektarbeit für den Bundeswettbewerb Fremdsprachen - Ein Arbeitsbericht. In: Praxis des neuspracblichen Unterrichts 49(3), 257-267. 
Wrobel, Arne (1995): Schreiben als Handlung - Überlegungen und Untersuchungen zur Theorie der Textproduktion. Tübingen: Niemeyer.

Links <letzter Zugriff: 20.09.2008>

http:/ / radiodaf.podspot.de/

http://skbpodcast.podspot.de/

http://verloren-in-berlin.podspot.de/

http://www.schulpodcasting.info

http://www.interaudio.org

http://spzwww.uni-muenster.de/ griesha/eps/wrt/ueb/index.html

i Interessante Beispiele für Lernerproduktionen finden sich unter http://radiodaf.podspot.de/ oder http://skbpodcast.podspot.de/ oder http://verloren-in-berlin.podspot.de/. Hinweise und Anregungen zum Erstellen eigener podcasts mit einem Schwerpunkt, Podcast im Fremdsprachenunterricht' bei Peuschel (2007), unter http://www. schulpodcasting.info. Zu interkultureller Radioarbeit vgl. Schwarzmeier \& Werner (2007) und www.interaudio. org.

ii $\mathrm{Zu}$ „Eigenschaften gesprochener Sprache“ vgl. einführend Fiehler et al. (2004).

iii Vgl. Edwards \& Lampert (1993); Kuckartz (2007); Mayring (2002).

iv Zu Revisionen in (muttersprachlichen) Schreibprozessen vgl. Wrobel 1995; Rau 1994; zur digitalen Übertragung schriftlicher Produktion in der Zweitsprache Deutsch vgl. Grießhaber (http://spzwww.uni-muenster.de/ griesha/eps/wrt/ueb/index. html.).

iv Mehr dazu vgl. das Themenheft „Mündliche Produktion in der Fremdsprache“ der Zeitschrift Fremdsprachen Lehren und Lernen (Henrici; Königs \& Zöfgen (Hrsg.) (2003)).

v Als Maße zur Bestimmung der Flüssigkeit mündlicher Äußerungen haben sich seit den 1980er Jahren für die Fremd- und Zweitsprachenforschung die Sprechrate (SR), die Anzahl der produzierten Silben pro Minute, und die Artikulationsrate, die Anzahl der produzierten Silben pro phonierte Minute einer Äußerung etabliert. Weitere Werte sind phonation-time-ratio (PTR) als der prozentuale Wert des Verhältnisses von phonierter zu nicht phonierter Zeit und die durchschnittliche Anzahl produzierter Silben innerhalb einer phonierten Sequenz zwischen zwei Pausen (mean lenght of runs, MLR) (vgl. Stevener 2003: 27ff). 


\title{
Kulturelles Lernen als Modifikation personaler Kulturkonstrukte - Eine Projektheuristik
}

\author{
Jan P. Pietzuch (Bielefeld)
}

\begin{abstract}
Die hier in ihrer Projektheuristik zu skizzierende Studie widmet sich auf Grundlage eines transdisziplinär konzipierten Theorie- und Methodendesigns der empirischen Erforschung individueller kultureller Lernprozesse im Kontext des Fremdsprachenerwerbs. In einem ersten Schritt positioniere ich Erkenntnisinteresse und Fragestellung des Projekts in relevanten Forschungsdiskursen, wobei verdeutlicht wird, dass seriöse kulturbezogene Lernprozessforschung nur über systematische(re) Bezüge auf Modelle und Forschungsergebnisse der Fremdsprachenerwerbsforschung sowie der neueren Sozial-, Kultur- und Bildungswissenschaften zu begründen ist. Entsprechend schlage ich eine funktional auf das empirische Design bezogene Refiguration dreier Kernkonzepte vor, in der ,Kultur‘, ,Subjekt' und ,Lernen“ mit Hilfe der analytischen Kategorien a) Diskursivität, b) Narrativität und c) Dialogizität als theoretische Konzepte aktualisiert und als empirische Konstrukte konkretisiert werden. Daraus leite ich ein vorläufiges heuristisches Modell zum kulturellen Lernen ab, skizziere dessen method(olog)ische Implikationen und stelle das hierbei zentrale Konzept der ,personalen Kulturkonstrukte zur Diskussion. Dieses operative Konzept und das zugeordnete analytische Inventar ermöglichen es, so die These, zwischen der hochgradigen Individualität kultureller Lernprozesse und deren prinzipieller Situiertheit in sprachlich-symbolisch prozessierten, sozialen Wissensordnungen zu vermitteln und kulturelles Lernen als Modifikation individuell verfügbarer Schematarepertoires empirisch zu erforschen.
\end{abstract}

\section{Einleitung}

Das Forschungsprojekt bezieht seine Relevanz einerseits aus Analysen außerwissenschaftlicher, globalgesellschaftlicher Problemlagen, die unter Verweis auf wirtschaftliche, politische und kulturelle Globalisierungsprozesse dem Sprachenund damit (inter-)kulturellen Lernen eine zentrale Bedeutung beimessen. Diesem vorwissenschaftlichen Begründungskomplex ist auch die Praxis des institutionalisierten Fremdsprachenunterrichts zuzuordnen, dessen landeskundliche Komponente sich oftmals weiterhin über deduktiv entwickelte und normativ begründete Modelle interkultureller Kompetenzen legitimiert, obgleich deren theoretische Grundannahmen wie auch deren Praktikabilität und ,Messbarkeit‘ zunehmend in Frage gestellt werden. Theoretisch ausreichend differenzierte und empirisch abgesicherte Modelle, v.a. auch zu individuellen kulturellen Lernprozessen, liegen für die 
Landeskunde des Faches Deutsch als Fremd- bzw. Zweitsprache (DaF/DaZ) bislang allenfalls in Ansätzen vor. Landeskundepraktiker/innen und die im Entstehen begriffene Fachwissenschaft, aber auch öffentliche Institutionen, wie z.B. das BMBF, melden hier dringenden Forschungsbedarf an. ${ }^{i}$

Zum anderen nimmt das Projekt seinen Ausgang in wissenschaftstheoretischen und methodologischen Entwicklungen, wie sie sich seit den 1990er Jahren in den Feldern der Sozial- und Kulturwissenschaften, in Ansätzen auch in der Fremdsprachenlehr-/lernforschung vollzogen haben. Die in diesen durch linguistic und cultural turns (vgl. Rorty 1967; Bachmann-Medick 2006) geprägten, zunehmend transdisziplinären Diskussionszusammenhängen geleistete Forschungs- und Reflexionsarbeit, v.a. die Rekonzeptualisierung grundlegender Begriffe und Kernkategorien (z.B. Kultur, Sprache, Subjekt, Lernen), bietet dem Forschungsfeld ,(inter)kulturelles Lernen' ein innovatives Potential, das bislang noch nicht im erforderlichen Maße aufgegriffen und auf theoretischer und/oder empirischer Ebene umgesetzt wird.

Das Forschungsprojekt widmet sich diesem Forschungsdesiderat und setzt sich zum Ziel, an der Entwicklung einer ebenso empirisch wie theoretisch fundierten Kulturdidaktik mitzuwirken, die sich zu wissenschaftlichen und realgesellschaftlichen Entwicklungen wie auch zur jeweiligen Fremd-/Zweitsprachen lernenden Person auf Augenhöhe positioniert.

\section{Positionierung im Diskussions- und Forschungsstand}

Die in gleichem Maße auf transdisziplinäre Theoriebildung und empirische Durchdringung des Gegenstands, fremdsprachenbezogenes kulturelles Lernen` zielende Ausrichtung der Studie erfordert eine Positionierung im Bereich des interkulturellen Lernens (2.1), im Feld der kulturwissenschaftlich orientierten Fremdsprachendidaktik und -forschung (2.2) sowie im Wissenschaftsbereich Kulturstudien DaF und damit aktuellen method(olog)ischen Diskursen der Sozial- und Kulturwissenschaften (2.3). Im Rahmen dieses Beitrags soll und kann dies nur skizzenhaft geschehen.

\subsection{Positionierung im Verhältnis zum ,Interkulturalitätsparadigma‘}

Auf thematischer Ebene nimmt das Projekt seinen ,fachhistorischen' Ausgang in den fremdsprachen- und landeskundedidaktischen Diskussionen zum sog., interkulturellen Lernen'. Dabei standen lange Zeit didaktisch-normative bzw. politischnormative Kompetenzmodelle und Zielbeschreibungen interkulturellen Lernens im Vordergrund der konzeptionellen Diskussion und didaktisch-methodischen Adaption. Hingegen erscheint die wissenschaftliche Auseinandersetzung mit (inter-) kulturellen Lernprozessen im deutschsprachigen Raum verhältnismäßig übersichtlich, obwohl insbesondere empirische Arbeiten zum , landeskundlichen` bzw. kultu- 
rellen Lernen seit längerem als ein sprachenübergreifendes Forschungsdesiderat gelten (vgl. u.a. bereits Bender-Szymanski \& Hesse 1993; Finkbeiner \& Koplin 2001). ii

Während v.a. im Bereich des Französischen als Fremdsprache in den letzten Jahren einige Arbeiten erschienen sind, die (inter)kulturelles Lernen in Spracherwerbs- und -vermittlungskontexten nicht aus einer didaktisch-normativen, sondern aus empirischer Perspektive untersuchen iii, bleibt das Feld einschlägiger Arbeiten im Bereich der DaF-Landeskunde auf die Erforschung sog. ,Deutschlandbilder von portugiesischen und spanischen (Iberische Lektorenarbeitsgruppe 1999; Spaniel 2004) bzw. japanischen Deutschlernenden (Grünewald 2005) sowie der Veränderung solcher Bilder bei russischen Deutschlerner/innen im Rahmen eines Schüleraustauschs (Ertelt-Vieth 2005) beschränkt.iv

Im Anschluss an die bereits von Knapp-Potthoff (1997) formulierten, von $\mathrm{Hu}$ (1999) und Altmayer (2006b) nochmals systematisch erweiterten Kritikpunkte nimmt das eigene Projekt eine kritisch-konstruktive Position gegenüber dem interkulturellen Paradigma ein, welche zum einen auf empirisch-methodischer Ebene, zum anderen auf theoretisch-methodologischer Ebene entwickelt wird. Hierbei dienen die Kernkonzepte ,Kultur', ,(Lerner-)Subjekt‘ und ,Lernen“ als analytische Fixpunkte zur Rekonstruktion einer diskursiven Transformation des Forschungsfeldes, in deren Folge sich die kategorialen Grundannahmen des klassischen Interkulturalitätsparadigmas in ihrer mitunter stark komplexitätsreduzierenden Funktion als nicht mehr zeitgemäß erweisen (vgl. 3). So ist auch in den internationalen Diskursen der neueren Fremdsprachenforschung und -didaktik eine deutliche Verlagerung der zentralen Forschungsfragen und -probleme zu beobachten. Nicht mehr ,interkulturelles (Fremdsprachen-)Lernen', sondern ,sprachliches Lernen als soziale Praxis in kulturell heterogenen Feldern' kann vorerst als pointierte Formulierung dieser Neuorientierung dienen (vgl. u.a. Phipps \& Gonzales 2004).

\subsection{Positionierung im Diskurs der kulturwissenschaftlich orientierten Fremdsprachendidaktik und -forschung}

In weitaus stärkerem Maße, als dies bislang in der ,Landeskunde ${ }^{6}$ des Faches DaF geschehen ist, werden in den sprachraumübergreifenden Diskussionen der Fremdsprachendidaktik und -forschung seit geraumer Zeit unterschiedliche kulturwissenschaftliche Konzepte und Ansätze auf ihre möglichen Potentiale für die fremdsprachenwissenschaftliche Theorie, Methodologie und Empirie befragt. Folgt man $\mathrm{Hu}$ (2007), kann inzwischen durchaus von einem kulturwissenschaftlichen Paradigma in der Fremdsprachenforschung gesprochen werden, dessen Produktivität in Bereichen der sprach-, kultur- und lernbezogenen Theoriebildung, der Weiterentwicklung empirischer Forschungsmethoden sowie der didaktisch-methodischen Reflexion eine pauschale Kritik am ,modischen Metadiskurs über letztlich fachfremde Themen'kaum mehr sinnvoll erscheinen lässt. 
Der heuristische Rahmen der eigenen Studie knüpft dabei v.a. an die sozial- und kulturtheoretische Rekonzeptualisierung des Zusammenhangs von individuellen Sprachlernprozessen, sozial-kommunikativen Praktiken und kulturellen Kontexten an, wie sie sich in der zunehmend kritischen Auseinandersetzung mit bis dato vorherrschenden, ,modernen' individual- und kognitionspsychologischen bzw. psycholinguistischen Ansätzen zum Spracherwerb abzeichnet.v Im Zuge der sich spätestens seit Firth \& Wagner (1997) intensivierten sog. cognitive-social debate vi und mit der Etablierung des ,soziokulturellen Paradigmas' der Spracherwerbsforschung, das aktuell gar als new mainstream SLA theory bezeichnet wird (Swain \& Deters 2007), sowie auch in verwandten Forschungsansätzen wie bspw. den language socialization studies (vgl. expl. Kramsch 2002), rücken soziale, kontextuelle und kulturelle Faktoren des Spracherwerbs, v.a. also auch der Zusammenhang von Sprachlernprozessen, soziokultureller Partizipation und personaler Identitätskonstruktion ins Zentrum des theoretischen und empirischen Interesses der neueren Fremdsprachenerwerbsforschung (vgl. u.a. Lave \& Wenger 1991; Wenger 1998; Lantolf 2000; Norton 2000; Block 2003; Watson-Gegeo 2004; Zuengler \& Miller 2006; Block 2007; Lantolf 2007).

Diese sozialkonstruktivistisch akzentuierte Umstellung des theoretischen und methodologischen Instrumentariums, die Verlagerung des Erkenntnisinteresses auf den Einfluss bspw. institutioneller und kultureller Settings, aber auch von Sprachund Kulturbiografien auf individuelle Lernprozesse, sowie die empirische Neuorientierung u.a. auf ethnographische, konversations- und diskursanalytische Studien (vgl. expl. Duff 2002; Kramsch 2000) zeigen schließlich, so die forschungsleitende These der Studie, deutliche Parallelen zur kulturwissenschaftlichen Wende in der Landeskunde des Faches DaF, wie sie sich derzeit insbesondere im Wissenschaftsbereich Kulturstudien DaF abzeichnet.

\subsection{Positionierung im Wissenschaftsbereich Kulturstudien DaF}

Im Zentrum der institutionellen und zunehmend auch diskursiven Etablierung des vergleichsweise jungen Wissenschaftsbereichs Kulturstudien DaF steht die kulturwissenschaftliche Transformation der herkömmlichen Landeskunde und die Herausbildung eines genuin kulturbezogenen fremdsprachenwissenschaftlichen Forschungsbereichs. Dabei wird den erheblichen theoretischen und forschungspraktischen Defiziten der DaF-Landeskundevii ein innovatives kulturwissenschaftliches Theorie- und Forschungsprogramm gegenübergestellt, das sich nicht mehr über das klassische Modell der Bezugswissenschaften und die Adaption von Inhalten, Gegenständen und Forschungsfragen anderer Disziplinen legitimiert, sondern sich über ,facheigene, d.h. aus der sozialen Praxis des Fremdsprachenlernens und lehrens stammende, Probleme und Erkenntnis leitende Interessen konstituiert, diese mittels (davon abhängiger) transdisziplinär konzipierter Forschungsinstrumentarien bearbeitet und gewonnene Erkenntnisse systematisch in die Praxis zurückführt (vgl. v.a. Altmayer 2004, 2007). Unter Berücksichtigung veränderter glo- 
balgesellschaftlicher, lebens- und damit auch sprachlehr-/lernpraktischer Problemlagen knüpfen die Kulturstudien darüber hinaus an eben jene transdisziplinären Diskussionszusammenhänge der neueren Kulturwissenschaften an, die auf die Notwendigkeit der Reformulierung klassischer Forschungsfragen und Erkenntnisinteressen und damit der Neubestimmung ihrer conceptual kits, d.h. theoretischer, methodologischer und empirischer Instrumentarien, verweisen (vgl. expl. Bachmann-Medick 2006).

Entsprechend basiert das von Claus Altmayer mit seiner Schrift Kultur als $\mathrm{Hy}$ pertext vorgelegte Forschungsprogramm einer kulturwissenschaftlichen Textanalyse auf einer Rekonzeptualisierung konstitutiver Kategorien. So wird u.a. das fremdsprachendidaktisch-landeskundliche Erkenntnisinteresse des ,Fremdverstehens zwischen empirisch-kognitionswissenschaftlichen, konstruktivistischen und normativ-hermeneutischen Ansätzen neu verortet und verstärkt an ,kulturelles Wissen angebunden. Damit wird Fremdverstehen als komplexe lernerseitige Fähigkeit verstanden, in der Auseinandersetzung mit fremdsprachlichen ,Texten` aller Art individuelle und/oder kulturell tradierte Muster der Welt- und Selbstdeutung zu reflektieren, zu relativieren sowie im Zuge einer , angemessenen' Sinn(re)konstruktion ggfs. zu modifizieren (vgl. dazu Altmayer 2004: 36ff.)

Das eigene Projekt schließt an Altmayers theoretische Grundlegung kulturwissenschaftlicher Forschungspraxis im Fach DaF insofern an, als es sich von positivistisch-szientistischen Positionen abgrenzt und - der hermeneutischen sozialwissenschaftlichen Tradition folgend - den ,sinnhaften Aufbau der sozialen Welt ${ }^{6}$ (Alfred Schütz) fokussiert. Das Forschungsinteresse gilt somit Subjekten vorgängigen und in/über Sprache sozial ,objektivierten' Wissens-, Erfahrungs- und Sinnstrukturen, die einer ,an sich' nicht erfahrbaren, sondern allein über ,Be-/Deutung' zugänglichen sozialen Wirklichkeit - und somit auch individuellen Sinngebungsund Deutungsprozessen - zugrunde liegen (vgl. z.B. Altmayer 2004: 112ff.).

Für den empirischen Fokus des Altmayerschen Ansatzes einer ,Kultur- als (Hyper-)Textanalyse ist konstitutiv, dass der Gegenstand ,Kultur ${ }^{6}$ bzw. dessen ,Operationalisierung ${ }^{\text {viii }}$ als Archiv oder Reservoire an kulturellen Deutungsmustern weder ein quantitativ-empirisch erklärbares, ,faktisches' Oberflächenphänomen, noch ein kognitionspsychologisch zu untersuchendes ,mentales ${ }^{6}$ Phänomen darstellt, wie es z.B. die kognitive Kulturanthropologie mit dem Konzept der ,cultural models' vorschlägt (vgl. Holland \& Quinn 1987). Vielmehr sind kulturelle Deutungsmuster nach Altmayer und im Anschluss an interpretative Kulturtheorien (vgl. Reckwitz 2000: 363ff.) als ,öffentliche` Phänomene und als Inhalte des kulturellen Gedächtnisses einer Sprachgemeinschaft, d.h. als textuell-medial repräsentierte und ,unterhalb der Textoberfläche' prozessierte Sinn- und Wissensstrukturen zu verstehen, die primär über qualitativ-hermeneutische Verfahren der Text- (und Medien-) Analyse rekonstruiert und damit für Lernende des Deutschen als Fremdsprache reflexiv verfügbar gemacht werden sollen. 
Damit kann ,kulturelles Lernen' als relationaler Prozess verortet werden zwischen der Ebene der Texte/Diskurse bzw. der dort präsupponierten kulturellen Deutungsmuster einerseits und der Ebene der empirischen Subjekte bzw. deren jeweils individuell aktualisierten kulturellen Deutungsschemata andererseits (siehe 4.3).

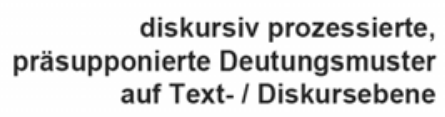

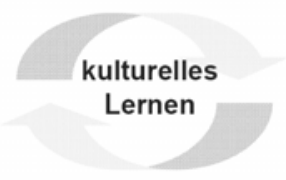

in Interaktion aktualisierte, ,personale' Deutungsschemata auf Rezipientenebene

Abb. 1: Verortung kulturellen Lernens.

Die i.w.S. textanalytische, auf die Rekonstruktion textuell-diskursiv prozessierter Deutungsmuster zielende, Ausrichtung des Altmayerschen Forschungsprogramms hat sich in kleineren Forschungsprojekten am Lehrstuhl Kulturstudien DaF und in einigen jüngeren Publikationen zu einer eigenständigen ,kulturwissenschaftlichen Diskursanalyse' weiter entwickelt und in Teilen empirisch bewährt (vgl. expl. Altmayer 2007).

Es fehlt jedoch bislang ein kompatibles method(olog)isches Instrumentarium, auf dessen Grundlage kulturelle Lernprozesse von (Lerner-)Subjekten unter Berücksichtigung je individueller, z.B. sprach- und kulturbiografischer, Ausgangs- und Bedingungslagen empirisch fundiert als prozessuale Aktualisierung, Differenzierung und Modifikation personaler Deutungsschemata in interpersonaler Kommunikation und Interaktion mit symbolisch-medial vermittelten Sinnwelten zu beschreiben wären.

\section{Erkenntnisinteresse und Fragestellung(en)}

Über die Positionierung in fremdsprachen- und kulturwissenschaftlichen Diskussionsständen und den dort formulierten (praxis- und wissenschaftsorientierten) Forschungsdesiderata lässt sich das erkenntnisleitende Interesse des Projekts als ein

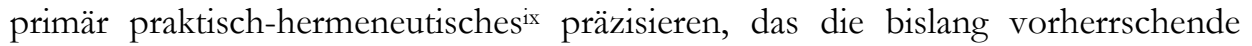
normative Perspektive auf den Gegenstand des (inter-)kulturellen Lernens überwindet und auf die empirische Deskription von ,tatsächlich'stattfindenden individuellen kulturellen Lernprozessen zielt. Dieses praxisorientierte Interesse ist jedoch allein im Rahmen seiner immanenten ,Perspektivengebundenheit', d.h. seiner spezifischen epistemologischen und methodologischen Prämissen, zu begründen und somit ohne ein gleichzeitiges theoretisches Erkenntnisinteresse nicht denkbar. Schließlich verfolgt das Projekt ein emanzipatorisches Interesse, indem es auf konzeptueller Ebene das prominente mentalistische Subjektmodell des ,(mono)kulturell determinierten' Lernenden auf ein ,kontextsensitives', neostrukturalistisches Akteurmodell umstellt (siehe 4.2). Zudem werden individuelle Prozesse der Refle- 
xion/Bewusstmachung, kulturbezogener ${ }^{6}$ Fremd- und Eigenpositionierungen und die als Bildungsprozess hypothetisch gesetzte Differenzierung von Deutungsoptionen auf Seiten der Lernenden als Erweiterung ihrer lebensweltlichen Handlungsoptionen verstanden (siehe 4.3).

Aus diesem Interessenkomplex leiten sich folgende Fragestellungen ab, wobei deren Abfolge die inhaltlichen Schwerpunkte des Projekts, deren Spezifität den Charakter einer explorativen Studie widerspiegelt:

(1) Was heißt ,kulturelles Lernen“ im Kontext spätmoderner, durch sprachlichkulturelle Heterogenität, Hybridisierung und neue Formen der Sozialisation/Subjektivation geprägter, Gesellschaften? Wie stellt sich kulturelles Lernen in sprach-, sozial-, kultur- und subjekttheoretischen Diskursen dar?

(2) D.h., mit Hilfe welches Theorien-, Begriffs- und Methodeninstrumentariums kann kulturelles Lernen im Rahmen aktueller kulturwissenschaftlicher Ansätze der Fremdsprachenforschung empirisch untersucht werden?

(3) Wie stellt sich individuelles kulturelles Lernen im Kontext des Fremd-/Zweitsprachenerwerbs unter Grundlegung des entwickelten heuristischen Frameworks auf empirischer Ebene dar? D.h., wie verändern sich die in diskursiven Sinnzuschreibungsprozessen jeweils applizierten Deutungsschemata oder ,Schematarepertoires' einzelner Individuen im Laufe der Zeit?

(4) Welche individuellen Faktoren (z.B. sprach- und kulturbiografische, ressourcenbezogene $e^{\mathrm{x}}$ und motivationale) erweisen sich in diesen Lernverläufen jeweils als konstitutiv?

(5) (Wie) Lassen sich die empirischen Ergebnisse der Einzelstudien fallvergleichend zu einem ,Prozessmodell mittlerer Reichweite‘ aggregieren, das als Grundlage für kulturdidaktische Konzeptionen dienen kann?

Aus dem o.g. praktisch-hermeneutischen Erkenntnisinteresse heraus wird zudem die Zielsetzung abgeleitet, mit Hilfe des Begriffs der ,personalen Kulturkonstrukte' ein heuristisches Konzept in die Diskussion einzuführen, das (als Effekt des cultural turn in den Kulturstudien DaF) weniger einen ,neuen', empirisch zu ergründenden ,Gegenstand' als vielmehr eine (metaphorische) Analysekategorie, einen ,operativen Begriff ${ }^{r i}$ darstellt, der es erlauben soll, Phänomene in das Blickfeld der Fremdsprachenforschung/Kulturstudien zu rücken, die bislang nur sekundär bedeutsam erscheinen (vgl. hierzu Bachmann-Medick 2006: 25ff.; siehe 4.3). Indem es unter prozessualen Gesichtspunkten insbesondere die Ebene der ,Rezipienten“ (s.o.) bzw. der aktiv sinnkonstituierenden Individuen fokussiert, soll das Konzept der ,personalen Kulturkonstrukte', vereinfacht gesagt, als komplementäres Modell zum (m.E. ebenso ,operativen') Ansatz der ,kulturellen Deutungsmuster verstanden werden, der sich bislang v.a. im Forschungsfeld kulturwissenschaftlicher Text- und Diskursanalysen bewährt hat. 


\section{Heuristischer Rahmen}

Dass der hier skizzierten empirischen Studie trotz ihres qualitativ-explorativen Charakters eine umfangreichere konzeptionelle Diskussion vorangestellt ist, mag eventuell verwundern. Nur zu bekannt scheint die in methodischen Diskussionen im Feld der qualitativen Sozialforschung bisweilen noch vorgetragene Forderung nach größtmöglicher theoretischer und methodischer Unvoreingenommenheit, nach konsequentem Hypothesenverzicht auf Seiten des Forschers. Diese Position hat sich jedoch u.a. durch die Infragestellung der ,klassischen` Dichotomie von Hypothesen testenden (quantitativen) und Hypothesen generierenden (qualitativen) Forschungsverfahren als nicht haltbar erwiesen. Auch qualitativ-explorative Forschung bedarf der expliziten Reflexion von theoretischem Vorwissen, das auf erkenntnistheoretischer Ebene hypothesenähnliche Funktionen erfüllt (vgl. Meinefeld 2007xii). Nicht nur wird die Explikation und Transparenz des theoretischen Vorverständnisses einer empirischen Studie mittlerweile als zentrales Gütekriterium qualitativer Forschung verstanden (vgl. Steinke 2007), auch gilt spätestens seit Glaser (1978) theoretische Sensibilität als Grundvoraussetzung der dichten Interpretation von Daten und der empiriebasierten Modellbildung (vgl. Strauss \& Corbin 1996).

In Form einer ,Projektheuristik' werden im Folgenden die theoretischen und methodologischen Prämissen der Studie skizziert, wobei sich dieses Reframing der konstitutiven Kategorien ,Kultur', ,Subjekt' und ,Lernen', wie bereits erwähnt, funktional auf deren Anwendung im Rahmen der empirischen Untersuchung bezieht. Aus diesem Grund rücken unter Berücksichtigung der oben explizierten erkenntnisleitenden Interessen insbesondere kulturwissenschaftliche Theorien- und Methodenkomplexe in den Blick, die sich aus der empirisch-analytischen Praxis heraus entwickelt oder sich aber bereits in anderen kulturbezogenen Forschungsfeldern empirisch bewährt haben.

\subsection{Kultur als ,Diskursfeld“ - Kultur- als Diskursanalyse}

Wie im Verweis auf veränderte lebensweltliche Ausgangslagen und aktuelle kulturtheoretische Diskussionen bereits verdeutlicht wurde, können die (mehr oder minder explizit verwendeten) monolithisch-essentialistischen Konzepte von ,Kultur oder gar ,Nationalkultur ${ }^{6}$ im Bereich der Landeskunde DaF als hochgradig problematisch und nicht anschlussfähig gelten. Das Projekt knüpft deshalb zunächst an den von Altmayer im Rahmen seines Ansatzes explizierten, wissens- und bedeutungsorientierten Kulturbegriff an, der in seiner Orientierung auf ein Archiv an überindividuellen Sinnstrukturen (kulturellen Deutungsmustern) auch weitgehend dem Grundkonsens des interpretativen Paradigmas der Kulturwissenschaften entspricht (vgl. auch Reckwitz 2000). In den letzten Jahren ist dieses Konzept im methodischen Anliegen, das deutungsmusteranalytische Verfahren weiterzuentwickeln, verstärkt mit diskursanalytischen Ansätzen, insbesondere dem der Wissenssoziologi- 
schen Diskursanalyse (vgl. Keller 2005) verknüpft worden. Neben dem dort entwickelten, innovativen analytischen Instrumentarium ${ }^{x i i}$ orientiert sich das eigene Projekt insbesondere an der Umstellung der Kernkategorie ,Kultur ${ }^{6}$ von einem Kulturbegriff, der ein „stabiles, tradiertes, den Akteuren vorgegebenes Signifikationssystem“ bezeichnet auf ein (metaphorisiertes) Konzept von Kultur als „dynamisches, konflikthaftes Diskursfeld“xiv, d.h. „als permanenter Aushandlungsprozess von symbolischen Ordnungen, Artefakten und angemessenen Praktiken [, der] nur vorübergehend sozial-räumlich feste Muster kristallisiert" (Keller 2003: 283).

Auch das Forschungsprogramm der wissenssoziologischen Diskursanalyse fokussiert als analytische Kernkategorie gesellschaftlich oder kulturell (vorübergehend) konventionalisierte Deutungsmuster, die individuelle und kollektive Erfahrungen organisieren und meist Vorstellungen (Modelle) angemessenen Handelns implizieren. Sie werden hier verstanden als ,grundlegende bedeutungsgenerierende Schemata, die durch Diskurse verbreitet werden und nahe legen, worum es sich bei einem Phänomen handelt" (Keller 2005: 238). Dabei verknüpft ein Diskurs nicht allein tradierte, im kollektiven Wissensvorrat gespeicherte Interpretationsschemata zu mehr oder minder komplexen Deutungsarrangements. Diskurse bzw. soziale Akteure vermögen auch ,neue Deutungsmuster zu generieren und auf der gesellschaftlichen Agenda zu platzieren“ (ebd.; Herv. i.O.). Deutungsmusteranalyse als Bestandteil von Diskursanalysen fokussiert also nicht nur die Rekonstruktion von Deutungsmustern in ihren historischen Erscheinungsformen (z.B. als Elemente des kulturellen Gedächtnisses), sondern ,sie betrachtet ihre Genese und ihre Veränderungen als Ergebnis der diskursiven Deutungsarbeit sozialer Akteure“ im Hier und Jetzt (ebd.).

Das von Keller vorgelegte Forschungsprogramm kann exemplarisch für eine Reihe diskurstheoretischer und -analytischer Ansätze stehen, die das methodologische und empirische Potential der Kategorie ,Diskurs' für kulturwissenschaftliche Fragestellungen hervorheben, auch wenn die vereinzelt geäußerte Forderung, den hochgradig belasteten Begriff der ,Kultur als analytische Kategorie durch den des ,Diskurses ${ }^{6} z u$ ersetzen, im Bereich der Fremdsprachenwissenschaften (noch) nicht zum Feld des ,Sag- und Denkbaren' zu rechnen ist (vgl. jedoch expl. Phipps \& Gonzales 2004). Jedoch ist festzuhalten, dass die analytisch orientierte Rekonzeptualisierung von Kultur als Diskursfeld eine je ,adaptive“ Operationalisierung des Konstrukts ,Kultur‘ in konkreten empirischen Forschungsprojekten ermöglicht und das umfangreiche Repertoire diskursanalytischer Methodiken der zu intensivierenden empirischen Forschungspraxis entscheidende Anstöße geben könnte.

Insbesondere auch, integrative ${ }^{6}$ Ansätze, die makro- und mikroanalytische, also diskurs- (i.e.S.) und konversationsanalytische Zugänge triangulieren (vgl. z.B. Hacking 2004; Jørgensen \& Philipps 2002; Te Molder \& Potter 2005; Wetherell 1998) erscheinen dabei in ihrer analytischen Überwindung der cartesianischen Dualität von ,autonomen Strukturen' und ,autonomen Subjekten'wegweisend. Das analytische Instrumentarium des eigenen Projekts orientiert sich entsprechend an dieser method(olog)ischen Haltung. 


\subsection{Menschenbilddiskussion und postcartesianische Subjektmodelle}

Dieser Schwerpunkt des eigenen heuristischen Frameworks greift zum einen die im Kontext der Menschenbilddiskussion der Fremdsprachenforschung formulierte Forderung auf, dass jede ernsthafte wissenschaftliche Beschäftigung mit sprachlich-kulturellen Lernprozessen sich ihrer impliziten anthropologischen Grundannahmen bewusst sein bzw. diese insbesondere im Rahmen einer empirischen Studie mit Fremdsprachen lernenden Individuen explizit machen sollte, um die Diskussion und rationale Kritik des zugrunde liegenden Subjektmodells zu ermöglichen (vgl. Kurtz 2003; Grotjahn 2005).

Zum anderen stellt sich auf Grundlage eines diskurstheoretisch reformulierten Kulturbegriffs und im Hinblick auf die Erforschung kultureller Lernprozesse einzelner Individuen die Frage nach dem Verhältnis von ,Kultur/Diskurs' und ,Subjekt' insofern neu, als die Mehrzahl diskursanalytischer Arbeiten - v.a. im Anschluss an Foucaults strukturalistisch geprägte ,Archäologie des Wissens' - in ihrem Interesse an den Formationsregeln und Bedingungen der Entstehung historisch-kollektiver Wissensordnungen vielmehr strukturtheoretisch als handlungsoder akteurtheoretisch orientiert sind; prägnant formuliert: den Status des ,sinnverstehenden Subjekts' dezentrieren (vgl. Reckwitz 2000: 262ff.). Das deutlichste Beispiel hierfür liefert wohl Foucault selbst, in dessen ,radikal antimentalistische[r] Theorie" diskursiver Praktiken kollektive Wissenscodes nicht mehr als mentale Phänomene erscheinen, sondern allein in den diskursiven Praktiken selbst angesiedelt sind - und das ,Subjekt ' damit ausschließlich als ,Subjektposition', die vom ,Diskurs' konstituiert wird, erscheint (ebd.).

Ein solches Verständnis des Subjekts als ,Effekt des Diskurses‘ scheint auf den ersten Blick kaum mit jenem „elaborativ-prospektiven“ Subjektmodell (vgl. 4.3) vereinbar, wie es im Rahmen der erwähnten Diskussion als reflektionsfähiges, intentional und v.a. autonom handelndes Subjekt von "reduktiv-implikativen“ Subjektmodellen abgegrenzt wird (vgl. expl. Grotjahn 2005). Die oft als ,Abschaffung' (miss)verstandene Infragestellung des autonomen Subjekts verdeutlicht z.B. auch Stäheli, wenn er resümierend postuliert, dass die Identität eines Subjekts „,nicht unabhängig von seiner Einschreibung in verschiedene diskursive Oberflächen und Dispositive gefasst werden [kann]“ - das Subjekt mithin „erst durch den Diskurs als Ort seines Sprechens, Fühlens und Handelns geschaffen [wird]“ (2000: 48). Ähnlich positioniert sich auch Jürgen Link, der die Annahme einer ,prädiskursiven Subjektivität" als naiven erkenntnistheoretischen Realismus und einen Rückfall hinter das Modell der „postdiskursiven Subjektivität“ für seriöse kultur- und diskurstheoretische Ansätze als praktisch kaum denkbar bezeichnet (vgl. DiazBone 2006). Das Projekt schließt sich dieser analytischen Sichtweise prinzipiell an, vertritt jedoch die u.a. von Stuart Hall (1996) konstruktiv gewendete Position, das die Relativierung bzw. ,Dezentrierung' des Subjektstatus' keineswegs in einer ,Abschaffung' des Subjekts mündet, sondern vielmehr ein nicht zu unterschätzendes 
Potential zur Rekonzeptualisierung des Subjektbegriffs, insbesondere für die Erforschung kultureller Lernprozesse, bietet.

I agree with Foucault that what we require here is ,not a theory of the knowing subject, but rather a theory of discursive practice' [...]. However, I believe that what this decentring requires [...] is not an abandonment or abolition of ,the subject', but a reconceptualization - thinking it in its new, displaced or decentred position within the paradigm (Hall 1996: 3).

Bei Hall erweist sich die kategoriale Umstellung des Konzepts der ,kulturellen Identität‘ auf ein Modell der ,multiplen kulturellen Identifikationen und Positionierungen' als wegweisende Reartikulation des Verhältnisses von Subjekt und diskursiven Praktiken: „[] dentities are [...] increasingly fragmented and fractured; never singular but multiply constructed across different, often intersecting and antagonistic, discourses, practices and positions“ (ebd.: 4). Dabei ist von entscheidender Bedeutung, dass hier der ,appellativen Macht' und ,Vorgängigkeit ${ }^{\star}$ der Diskurse ein Modell gegenübergestellt wird, das das Subjekt insofern zumindest partiell rehabilitiert, als es ihm - unter der Bedingung seiner multiplen Positionierung und Konstitution durch Diskurse - die prinzipielle Fähigkeit zuschreibt, sich dieser Positionierungsprozesse bewusst $\mathrm{zu}$ werden und sich in Form intentionalen sprachlichsemiotischen Handelns selbst in diesen und anderen Diskursen zu positionieren, auch wenn dies evtl. nicht mehr ,autonom“ geschieht. ${ }^{\mathrm{xv}}$ In diesem Sinne: „The concept of identity deployed here is [...] not an essentialist, but a strategic and positional one" (ebd.).

Für eine weitere Konkretisierung der heuristischen Perspektive auf (Lerner-) ,Subjekt ${ }^{6}$ und ,Subjektivität ${ }^{6}$ greift das Projekt - unter primär empirischfunktionalen Gesichtspunkten - schließlich auf drei (Akteur-)Modelle aus der sozialkonstruktivistischen Psychologie zurück, die den ,Einbau' der Subjektperspektive in die oben skizzierte diskursanalytischen Fassung ,des Kulturellen' weiterführen. An allen drei Modellen - dem narrativen Selbst (Polkinghorne 1988; Bruner 1990; Schwerdtfeger 1996), dem diskursiven bzw. positionalen Selbst (Davies \& Harré 1990; Harré \& van Langenhove 1999) und dem dialogischen Selbst (Hermans \& Kempen 1993; Hermans 2001) - interessiert dabei neben ihren methodischen Implikationen (siehe 5) insbesondere die zwar jeweils spezifische, doch immer sozialepistemologische Perspektive auf das Verhältnis von vorgängigen überindividuellen Wissensund Sinnstrukturen, von sprachlicher Interaktion und der aktiven Konstruktion von Sinn, Bedeutung, Wissen und Identität in \& durch Sprache. Was damit gemeint ist, sei exemplarisch am Modell des narrativen Selbst bzw. der Kategorie des ,Narrativen' mit folgendem Zitat erläutert:

[1] $\mathrm{t}$ is through narrativity that we come to know, understand, and make sense of the social world, and it is through narratives and narrativity that we constitute our social identities. [W] hether we are social scientists or subjects of historical research, $[\ldots]$ all of us come to be who we are (however ephemeral, multiple and 
changing) by being located or locating ourselves (usually unconsciously) in social narratives rarely of our own making (Somers 1994: 606).

Bevor angedeutet werden kann, wie die Kategorien der Dialogizität, Narrativität und Diskursivität in ihrer epistemologischen Dimension dem heuristischen Konzept zum kulturellen Lernen - und in ihrer analytisch-methodischen Dimension auch dem empirischen Forschungsdesign - zugrunde liegen, soll die projekteigene ,Subjektheuristik' vorläufig präzisiert werden: Das Projekt stellt dem (zumindest im landeskundlichen Kontext) weiterhin prominenten, mentalistischen Modell des kulturell determinierten, (v.a auch unbewusst) in kulturspezifischen Mustern ,denkenden' und handelnden Lernenden ein diskursanalytisches und postkognitivistisch informiertes Akteurmodell des Lernsubjekts als relational-dialogisches, multipositionales Selbst gegenüber, dessen Subjektivität - mithin dessen, individueller Zugang zum Kulturellen' - sich aus einer Vielzahl von vor-/bewussten diskursiven Positionierungsprozessen sowie auf Grundlage vor-/bewusster, diskursiv prozessierter Muster der Welt- und Selbstdeutung formiert. „Die Vielfältigkeit gesellschaftlicher Diskurse ist denn auch die Vielfältigkeit des Subjekts“ (Stäheli 2000: 48). Damit vertritt das Projekt ein Menschenbild, das - wie Hu formuliert - „Subjekte nicht als organismisch determinierte, wenngleich emergente Systeme auffasst, sondern deren Geltungsansprüche und Selbstdeutungsmöglichkeiten sowie lebensweltliche, historische, lokale und ökologische Einbettung ernst nimmt" (2002: 170), und somit ebenfalls den elaborativ-prospektiven Subjektmodellen zugeordnet werden kann.

\subsection{Personale Kulturkonstrukte und kulturelles Lernen}

Der sozialkonstruktivistische Grundtenor der bisherigen Ausführungen macht ersichtlich, dass es sich (auch) bei dem Begriff der ,personalen Kulturkonstrukte nicht um einen ,neuartigen', in einem ,natürlichen' Kontext ,vorliegenden' und dem Forschenden direkt zugänglichen, Gegenstand handeln kann, den bislang noch niemand entdeckt hat und den es nun in positivistischer Manier zu definieren, zu operationalisieren und zu untersuchen gilt. Vielmehr stellt dieses Konzept, wie erwähnt, einen operativen Begriff dar, auf dessen Grundlage individuelle Sichtweisen auf kulturelle Phänomene aus qualitativ-empirischer und prozessorientierter Perspektive neu in den Blick genommen werden sollen. Vereinfacht formuliert: dem Begriff der ,kulturellen Identität' als diskursives Beobachterkonstrukt des Fachdiskurses sollen individuelle kulturbezogene Deutungs- und Wissensstrukturen im oben skizzierten Sinne als Teilnehmerkonstrukt, eben als ,personales Kulturkonstrukt gegenüber gestellt werden.

Im Rahmen der method(olog)ischen Konzeption des Untersuchungsdesigns setzt sich das eigene Projekt deshalb kritisch mit dem Forschungsprogramm Subjektive Theorien (expl. Groeben et al. 1988; Scheele \& Groeben 1998) auseinander, das im deutschsprachigen Feld der Fremdsprachenforschung als einflussreichste Metho-

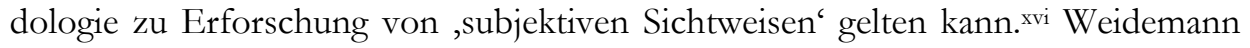


(2004) unternimmt als m.W. einzige Arbeit, die das FST der empirischen Rekonstruktion (inter)kultureller Lernprozesse zugrunde legt, zugleich eine theoretisch und empirisch begründete Problematisierung des FST in seiner Anwendung auf kulturund lernbezogene Fragestellungen. In vergleichbarer Weise münden erste konzeptuelle Versuche, diesen Forschungsansatz für das eigene Projekt fruchtbar zu machen, in einer kultur- und lern-/bildungstheoretischen Revision des FST, auf deren Grundlage das alternative Konzept der personalen Kulturkonstrukte begründet wird. Vorläufig - und wohlgemerkt im Rahmen der i.w.S. diskurstheoretisch informierten Projektheuristik - soll ein ,personales Kulturkonstrukt' charakterisiert werden als ein je individuell zur Verfügung stehendes Repertoire an (kulturellen) Deutungsmustern, Basis-Erääblungen und Ich-Positionen zu einem themenspezifischen Wissenskomplex,

- das in Form generischer Wissensstrukturen vorliegt und in der verstehensorientierten Interaktion mit Texten/Diskursen kontext- und situationsabhängig als spezifische Wissensstruktur aktualisiert wird;

- das dem Individuum als Grundlage des ,Aushandlungsprozesses ‘ zwischen subjektiv befriedigenden und dem Text/der Situation/der Interaktion ,angemessenen'Sinnzuschreibungen dient;

- das ,soziobiografisch“ konventionalisiert und u.a. von der individuellen sprachlich-kulturellen Biografie, dem individuellen Relevanzsystem und der individuellen Ressourcenlage abhängig ist;

- dessen Einzelkomponenten (Deutungsmuster, Ich-Positionen) relational-dialogisch strukturiert und narrativ eingebettet sind, jeweils Handlungsdispositionen (Modelle angemessenen Handelns) aufweisen und damit der Reflexion und Versprachlichung potentiell zugänglich sind.

Die mit diesem Konzept verbundenen theoretischen Umstellungen sollen und können an dieser Stelle nicht im Einzelnen erörtert werden, zumal sich die Praktikabilität einer solchen diskursanalytischen Perspektive auf Kultur, (Lerner-)Subjekt und kulturelle Lernprozesse erst in der empirischen Umsetzung erweisen wird. So wird u.a. nur forschungspraktisch zu ergründen sein, inwiefern die metho$\mathrm{d}(\mathrm{olog})$ ische Triangulation der Rekonstruktion von ,kognitiven Strukturen bzw. Schemata' und deren Transformation (z.B. über longitudinal eingesetzte ConceptMapping-Verfahren, vgl. z.B. Hilbert \& Renkl 2007) mit der diskurs-/konversationsanalytischen Untersuchung der interpretative repertoires (vgl. Potter \& Wetherell 1987) und der ,diskursiven Praxis‘ der Untersuchungspartner/innen (vgl. Edwards 1994) in der Auseinandersetzung mit Diskursfragmenten neue Perspektiven auf den Gegenstand individueller kultureller Lernprozesse eröffnen könnte (siehe 5).

In der Verortung ,kulturellen Lernens' zwischen Diskursen bzw. der diskursiven Konstruktion von sinnhafter Wirklichkeit einerseits und Subjekten bzw. individuellen Sinnzuschreibungen und Verstehensprozessen andererseits (vgl. 2.3) 
rekurriert das Projekt auf die von Altmayer aus seinem kulturtheoretischen Ansatz abgeleitete und bereits auf die empirische Umsetzung fokussierte Begriffsbestimmung:

Von ,kulturellem Lernen' soll [...] dann die Rede sein, wenn Lerner des Deutschen als Fremdsprache in der und veranlasst durch die Auseinandersetzung mit deutschsprachigen ,Texten' über die ihnen verfügbaren Deutungsmuster reflektieren und diese so anpassen, umstrukturieren, verändern oder weiterentwickeln, dass sie den kulturellen Deutungsmustern, von denen die Texte Gebrauch machen, weit gehend entsprechen, sie in die Lage versetzen, diesen Texten einen kulturell angemessenen Sinn zuschreiben und dazu angemessen (kritisch oder affirmativ) Stellung nehmen zu können (2006b: 256).

Im Rahmen der eigenen Projektheuristik wird das hier komplementär konzipierte Verhältnis von ,Verstehen' als aktiver Sinnkonstruktion des Individuums in der Interaktion von ,bottom up'- und ,top down'-Prozessen und von ,Verstehen' als dialogisch-normativem Verständigungsprozess in zweifacher Hinsicht fruchtbar gemacht. Auf einer deskriptiv-analytisch orientierten Ebene kann so neben dem Rückgriff auf diskursanalytische Instrumentarien u.a. an die empirische Textverstehensforschung, insbesondere auch an neuere soziokognitiv bzw. soziokulturell orientierte, auf Interaktionen fokussierte Forschungsdesigns aus dem Feld der conceptual change theory (vgl. expl. Säljö 1999) angeknüpft werden. Mit der deutlichen Betonung der reflexiven, die Prämissen eigenen Deutens und Handelns kontinuierlich hinterfragenden, Komponente wird jedoch gleichzeitig auf eine zweite, normativ-ethische Ebene verwiesen, auf der kulturelles Lernen seiner Komplexität gemäß als sprachlich-semiotisch figurierter Bildungsprozess sui generis theoretisch zu beschreiben und empirisch zu erforschen ist. In welcher Weise die hier skizzierte Perspektive dabei mit aktuellen erziehungswissenschaftlichen Debatten um eine Neufassung des Bildungsbegriffs konvergiert, kann vorerst nur exemplarisch im Verweis auf Arbeiten von Rainer Kokemohr und Hans-Christoph Koller angedeutet werden, in deren Annäherung an eine ,Theorie transformatorischer Bildungsprozesse“ zur Fundierung empirischer Bildungsforschung ein Verständnis von Bildungsprozessen als „Transformationen von Grundfiguren des Welt- und Selbstverhältnisses“" (Koller 2007: 69) entwickelt wird (vgl. Koller et al. 2007; aber auch Holzkamp 1995; Faulstich \& Ludwig 2004; Lüders 2004). xvii

In diesem Sinne sollen individuelle kulturelle Lernprozesse als relationale, narrative und diskursive Konstruktion/Modifikation personaler Kulturkonstrukte verstanden werden, wobei die oben eingeführten Kategorien der Dialogizität, der Narrativität und Diskursivität hier erneut aufgegriffen werden, um die sozialepistemologischen Grundlagen, d.h. die Situiertheit jedes (kulturellen) Lern- und Bildungsprozesses in vorgängigen kollektiven Wissensstrukturen und -ordnungen zu betonen. Gleichzeitig werden sie als ,Weisen' der aktiven (deutenden) Aneignung von und Auseinandersetzung mit je spezifisch symbolisch repräsentierten Wirklichkeiten interpretiert. Das folgende Modell versucht schließlich auch zu verdeutlichen, wie Dialogizität, Narrativität und Diskursivität als analytische Kategorien jeweils methodische 
,Zugänge ${ }^{`}$ zu Forschungs- und Lehr-/Lerngegenständen eröffnen, zudem mittelfristig als ,Strategien` zur Erschließung diskursiv prozessierter Selbst- und Weltsichten bzw. zur Aktualisierung und Restrukturierung eigener Musterrepertoires (Schemata, Basis-Erzählungen, Ich-Positionen) reformuliert und erforscht werden könnten.

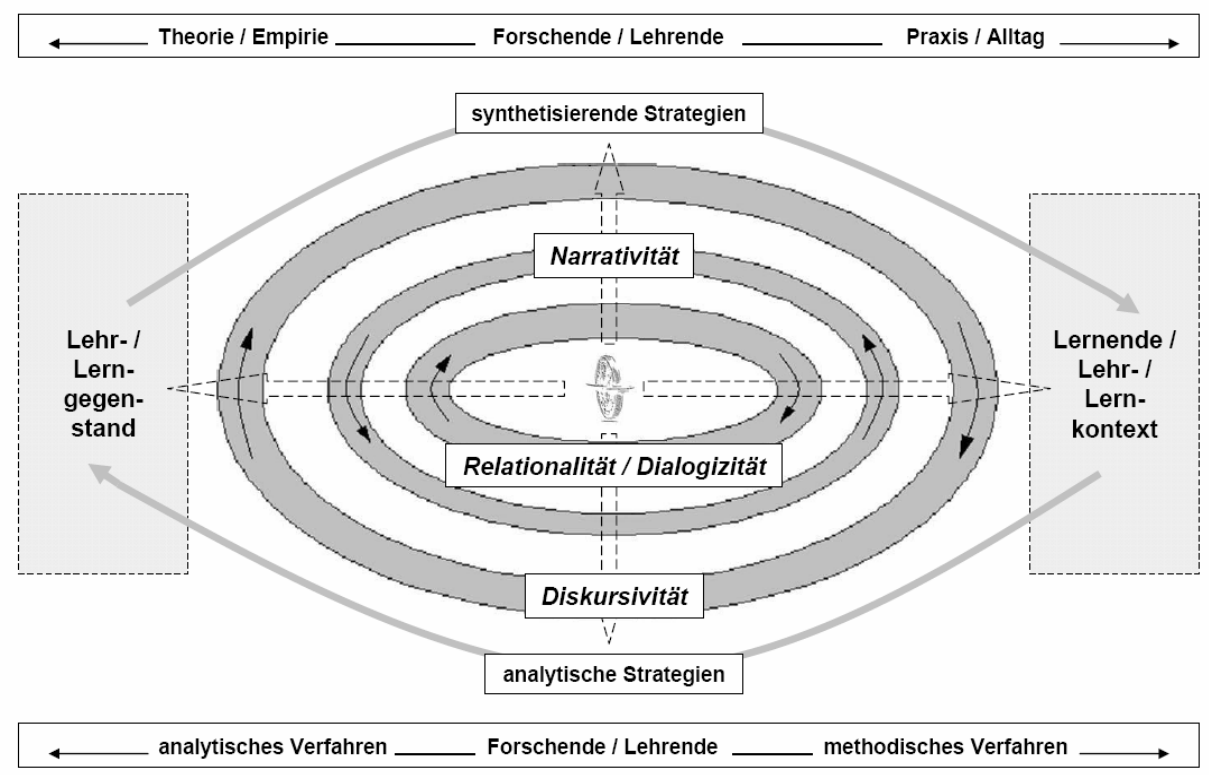

Abb. 2: Heuristisches Modell zum kulturbezogenen Lernen und Forschen.

\section{Empirisches Design}

Aus dem soweit skizzierten Erkenntnisinteresse und methodologischen Vorverständnis leitet sich ein ,hybridmethodisches' (Theorien und Methoden triangulierendes) Forschungsdesign ab, das sich am Modell einer fallvergleichenden, qualitativ-empirischen Longitudinalstudie (vgl. expl. Ortega \& Iberri-Shea 2005) orientiert und deren Ziel darin besteht, kulturelle Lernprozesse Deutsch lernender Individuen als Konstruktion und Transformation personaler Kulturkonstrukte in der Auseinandersetzung mit einem thematisch definierten ,Diskurs' ${ }^{6} \mathrm{zu}$ beschreiben. Weiterführend ist beabsichtigt, diese individuellen Konstrukttransformationen (,Lernverläufe) vergleichend aufeinander zu beziehen und zu einem datenbegründeten ,Modell mittlerer Reichweite‘ zu aggregieren. Unter Berücksichtigung der Kernfragestellung, auf Grundlage welcher individuell verfügbaren Musterrepertoires einzelne Individuen einem Diskurs als ,Gesamtdeutungsgegenstand' Sinn zuschreiben, welche impliziten Wissensstrukturen (kulturelle Deutungsmuster) dieser Diskurs seinerseits ,präsupponiert ${ }^{\star}$ und schließlich, wie sich die individuellen 
Musterrepertoires im Verlauf der deutenden Auseinandersetzung mit diesem Diskurs verändern, setzt sich die Gesamtstudie aus zwei empirischen Teilstudien zusammen - die sich, vereinfacht gesagt, jeweils auf die ,Struktur-' und die ,Akteurebene' beziehen. Sie zielen damit zum einen auf die diskursanalytisch zu rekonstruierenden Deutungsmuster, Subjektpositionen und Story-Lines auf der Text/Diskursebene, zum anderen auf die konversations- und diskursanalytisch zu untersuchenden Deutungsmuster, Basis-Erzählungen und Ich-Positionen auf der Ebene der Rezipienten ab. Zur Verdeutlichung dient folgendes Schema, das die jeweiligen Teilstudien und mehrmethodischen Zugriffe auf die Daten darstellt:

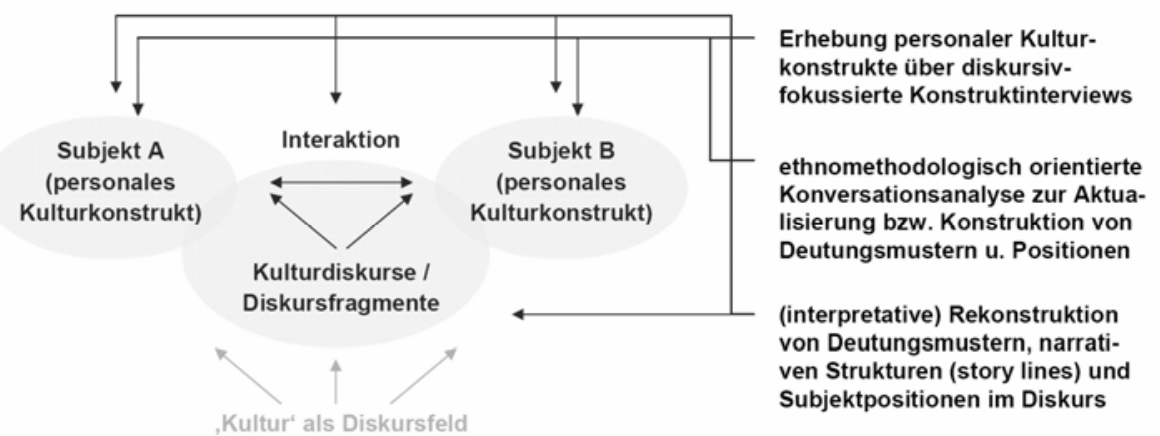

Abb. 3: Schema zum mehrmethodischen empirisch-diskursanalytischen Zugriff.

Als thematischer Rahmen (Kulturdiskurs) für die diskursanalytische Teilstudie (I) qualifiziert sich u.a. aus zeitgeschichtlichen und fachlichen Gründen der Diskurs des Neuen Patriotismus', wie er insbesondere im Kontext der Fußballweltmeisterschaft 2006, aber auch im mittelfristigen Vor- und Nachfeld eine spezifische Wirkung innerhalb und außerhalb der deutschsprachigen Diskursgemeinschaft entfaltet hat. Ziel der Analysen ist, wie erwähnt, die Aufbereitung des ,Gesamtdeutungsgegenstandes', d.h. die diskursstrukturierenden, meist in ,Erzählungen' eingebundenen, impliziten Muster und Positionen zu rekonstruieren und für die UntersuchungspartnerInnen im Rahmen der empirischen Teilstudie (II) reflexiv verfügbar zu machen. Das den Diskurs ,repräsentierende' Gesamtkorpus ${ }^{x v i i}$ setzt sich aus vier Teilkorpora zusammen, die jeweils in sog. Basistexte (zentrale Schlüsseltexte), Metatexte (kommentierende Beiträge) und Hintergrundtexte (zur sozial- und kulturgeschichtlichen Kontextualisierung) gegliedert sind, wobei insbesondere die Schlüsseltexte einer detaillierten Mikroanalyse mittels sequenz- und inhaltsanalytischer Verfahren sowie einer funktionalen Analyse der sprachlich-semiotischen Mittel (Bilder, Metaphorik, 'markierte' Lexik, Argumentationsmuster etc.) unterzogen werden. ${ }^{\text {xix }}$ 
1. Korpus Hörfunk: „Schwarz-Rot-Gold“(Sendereihe Deutschlandfunk 2006);

2. Korpus Medien: „Du bist Deutschland!“- Kampagne (2005);

3. Korpus Film: „Deutschland - ein Sommermärchen“(Sönke Wortmann, 2006);

4. Korpus Musik: „Wir sind Wir“(Peter Heppner \& Paul van Dyk); "Es ist was es ist" (Mia).

Für die Erstellung des Gesamtkorpus' ist v.a. maßgeblich, dass ausgewählte Diskursfragmente als textuelle Grundlage der empirischen Studie (II) dienen sollen. Die Auswahl der Schlüsseltexte erfolgt somit auch unter motivationalen bzw. rezipientenorientierten Gesichtspunkten.

Die sich derzeit in Arbeit befindliche empirische Teilstudie (II) soll an dieser Stelle in Form einer schematischen Darstellung der einzelnen Datenerhebungsphasen und der jeweiligen methodischen Zugriffe skizziert werden.

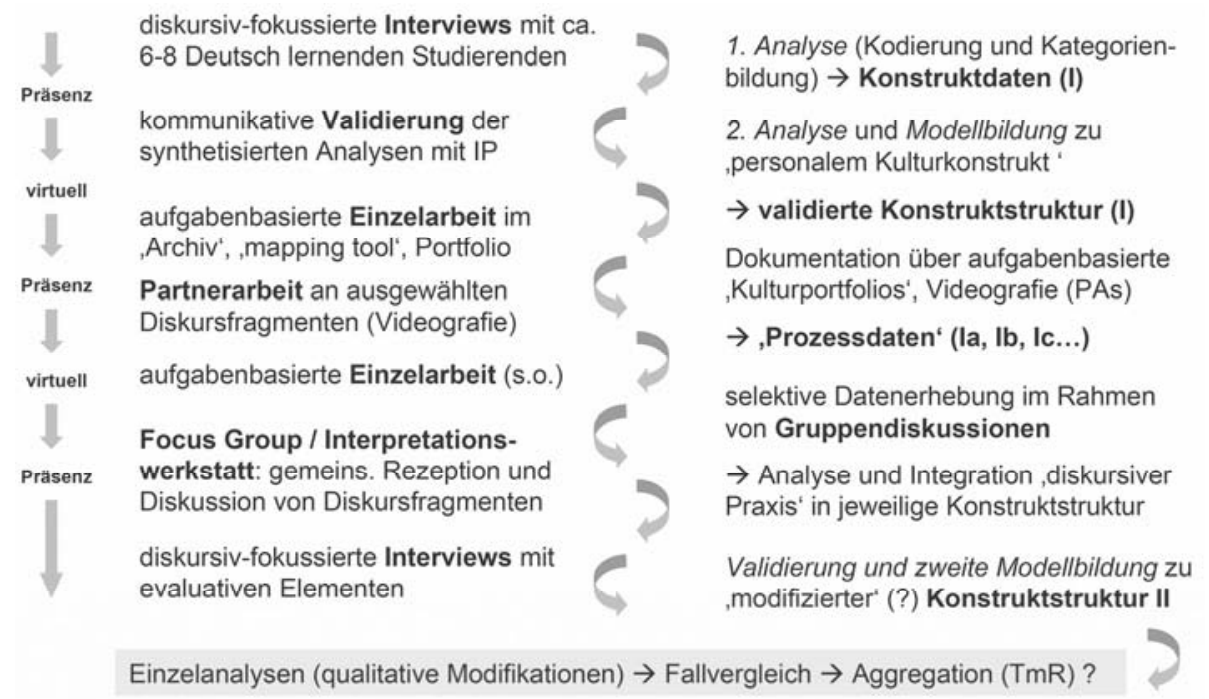

Abb. 4: Empirische Teilstudie II - Phasen und Verfahren der Datenerhebung.

$\mathrm{Zu}$ den zentralen Aspekten dieses Verfahrens gehört die Erhebung unterschiedlicher Datenformate, die vorläufig benannt sind als (a) Konstruktdaten, (b) Prozessdaten und (c) Interaktionsdaten - wobei unter (a) zum einen mündliche Daten aus den zwei diskursiv-fokussierten Konstruktinterviews (Ullrich 1999; König 1990), zum anderen auch visuelle Daten zum personalen Konstrukt fallen, die mittels mapping software als elektronische concept maps erhoben werden. Als (b) Prozessdaten dienen primär jene concept maps, die von den Untersuchungspartner/innen (UP) selbstständig während des ca. vierwöchigen Untersuchungszeitraumes bearbeitet und aktualisiert werden. Im Archiv (Lernplattform) setzen sich die UP mit einem Teil der in Studie (I) analysierten und selektierten Materialien (Texte, Bilder, Plakate, Videos, 
Musik etc.) auseinander und dokumentieren ihre Arbeit mit einem aufgabenbasierten ,Kulturportfolio'. Unter (c) fallen schließlich die Daten aus den videografierten Partnerarbeiten, sowie die Daten aus der Gruppendiskussion, in der ein Diskursfragment (z.B. ein Musikvideo) diskutiert wird. Gemäß der beabsichtigen Triangulation von empirischer Textverstehensforschung (,kognitive Strukturen) und Diskursanalyse (,diskursive Praxis) soll diesen Interaktionsdaten in den noch ausstehenden Analysen ein besonderes Augenmerk zukommen.

\section{Ausblick}

Dem hier skizzierten Forschungsprojekt liegen zwei Zielperspektiven zugrunde, die sich jeweils auf die Bereiche der Forschung und Lehre im Feld der kulturwissenschaftlich fundierten Fremdsprachenlehr-/lernforschung und -praxis beziehen. Zum einen soll die funktionale, d.h. auf, Operationalisierung ${ }^{6}$ und Anwendung fokussierte, Aktualisierung zentraler kultur-, subjekt- und lerntheoretischer Kernkonzepte und deren empirische Umsetzung - im Sinne des methodologischen Mehrwerts - einen Beitrag zur Weiterentwicklung des Diskussions- und Forschungsfeldes des fremdsprachenbezogenen (inter-)kulturellen Lernens leisten.

Zum anderen zielt das Forschungsprojekt - im Sinne des praktischen Mehrwerts - darauf ab, mit den Ergebnissen der Fallstudien und dem evtl. zu entwickelnden Prozessmodell die empirisch und kulturwissenschaftlich fundierte Diskussion curricularer und didaktisch-methodischer Ansätze zur sog. ,Landeskunde' im Bereich DaF/DaZ zu beleben und damit mittelfristig auf außer/akademische Lehr-/Lernkontexte Einfluss zu nehmen. Schließlich könnte ein prozessdaten- (statt kompetenz-)basiertes Modell auch in der Diskussion zur Evaluation sog. interkultureller Kompetenzen als Korrektiv wirken und gegenüber einem unter ethischen Gesichtspunkten höchst bedenklichen Abtesten von Persönlichkeitsprofilen alternative Wege aufweisen.

\section{Literatur}

Altmayer, Claus (2003): Deutsch als Fremdsprache und Kulturwissenschaft. In: Altmayer, Claus \& Forster, Roland (Hrsg.): Deutsch als Fremdsprache: Wissenschaftsanspruch -

Teilbereiche - Bezugsdisziplinen. Frankfurt a.M. [u.a.]: Peter Lang , 109-134.

Altmayer, Claus (2004): Kultur als Hypertext. Zu Theorie und Praxis der Kulturwissenschaft im Fach Deutsch als Fremdsprache. München: Iudicium.

Altmayer, Claus (2006): Kulturelle Deutungsmuster als Lerngegenstand. Zur kulturwissenschaftlichen Transformation der Landeskunde. In: Fremdsprachen Lehren und Lernen 35, 44-59.

Altmayer, Claus (2007). Kulturwissenschaftliche Diskursanalyse im Kontext des Faches Deutsch als Fremdsprache - Ziele und Verfahren. In: Redder, Angelika (Hrsg.): 
Diskurse und Texte. Festschrift für Konrad Ehlich zum 65. Geburtstag. Tübingen: Stauffenburg, 575-584.

Bachmann-Medick, Doris (2006): Cultural Turns. Neuorientierungen in den Kulturwissenschaften. Reinbek: Rowohlt.

Bechtel, Mark (2003): Interkulturelles Lernen beim Sprachenlernen im Tandem. Eine diskursanalytische Untersuchung. Tübingen: Narr.

Bender-Szymanski, Dorothea \& Hesse, Hermann-Günter (1993): Interkulturelles Lernen: Ein psychologischer Zugang über Alltagstheorien von Lehrern und Schülern. In: Unterrichtswissenschaft 21(2), 147-166.

Block, David (2003): The social turn in second language acquisition. Edinburgh: Edinburgh University Press.

Block, David (2007): Second Language Identities. London \& New York: Continuum.

Bourdieu, Pierre (1983): Ökonomisches Kapital, kulturelles Kapital, soziales Kapital. In: Kreckel, Reinhard (Hrsg.): Soziale Ungleichheiten. Göttingen: Schwartz, 183-198.

Breidbach, Stephan (2007): Bildung, Kultur, Wissenschaft. Reflexive Didaktik für den bilingualen Sachfachunterricht. Münster [u.a.]: Waxmann.

Bröckling, Ulrich (2007): Genealogie der Subjektivierung - ein Forschungsprogramm. In: Ders.: Das unternebmerische Selbst. Soziologie einer Subjektivierungsform. Frankfurt a.M.: Suhrkamp, 19-45.

Bruner, Jerome (1990): Acts of Meaning. Cambridge [u.a.]: Harvard University Press.

Brunzel, Peggy (2002): Kulturbezogenes Lernen und Interkulturalität. Zur Entwicklung kultureller Konnotationen im Fran₹ösischunterricht der Sekundarstufe I. Tübingen: Narr.

Busse, Dietrich \& Teubert, Wolfgang (1994): Ist Diskurs ein sprachwissenschaftliches Objekt? Zur Methodenfrage der historischen Semantik. In: Dies. \& Hermann, Fritz (Hrsg.): Begriffsgeschichte und Diskursgeschichte. Opladen: Westdeutscher Verlag, 10-28.

Davies, Bronwyn \& Harré, Rom (1990): Positioning. The Discursive Production of Selves. In: Journal for the Theory of Social Behaviour 20(1), 45-63.

Diaz-Bone, Rainer (2006): Operative Anschlüsse. Zur Entstehung der Foucaultschen Diskursanalyse in der Bundesrepublik. Jürgen Link im Gespräch mit Rainer DiazBone. In: Forum Qualitative Sozialforschung 7(3) [http://www.qualitative-research.net; 20.09.2008].

Duff, Patricia A. (2002): The Discursive Co-Construction of Knowledge, Identity, and Difference. An Ethnography of Communication in the High School Mainstream. In: Applied Linguistics 23(3), 289-322.

Edwards, Derek (1994): Script Formulations. An Analysis of Event Descriptions in Conversation. In: Journal of Language and Social Psychology 13(3), 211-247.

Ertelt-Vieth, Astrid (2005): Interkulturelle Kommunikation und kultureller Wandel. Eine empirische Studie zum russisch-deutschen Scbüleraustausch. Tübingen: Narr. 
Faulstich, Peter \& Ludwig, Joachim (2004): Lernen und Lehren - aus "subjektwissenschaftlicher Perspektive". In: Dies. (Hrsg.): Expansives Lernen. Baltmannsweiler: Schneider-Verlag Hohengehren, 10-28.

Finkbeiner, Claudia \& Koplin, Christine (2001): Fremdverstehensprozesse und interkulturelle Prozesse als Forschungsgegenstand. In: Müller-Hartmann, Andreas \& Schocker-v. Ditfurth, Marita (Hrsg.): Qualitative Forschung im Bereich Fremdsprachen lehren und lernen. Tübingen: Narr, 114-136.

Firth, Alan \& Wagner, Johannes (1997): On Discourse, Communication, and (Some) Fundamental Concepts in SLA Research. In: The Modern Language Journal 81(3), 285300 .

Gee, James P. (2005). An Introduction to Discourse Analysis. Theory and Method. London [u.a.]: Routledge.

Glaser, Barney (1978): Theoretical Sensitivity. Mill Valley, CA: Sociology Press.

Groeben, Norbert; Wahl, Diethelm; Schlee, Jörg \& Scheele, Brigitte (1988): Forschungsprogramm Subjektive Theorien. Eine Einfübrung in die Psychologie des reflexiven Subjekts. Tübingen: Francke.

Grotjahn, Rüdiger (2005): Subjektmodelle. Implikationen für die Theoriebildung und Forschungsmethodologie der Sprachlehr- und Sprachlernforschung. In: Zeitschrift für Fremdsprachenforschung 16(1), 23-56.

Grünewald, Matthias (2005): Bilder im Kopf. Eine Longitudinalstudie über die Deutschland- und Deutschenbilder japanischer Deutschlernender. München: Iudicium.

Habermas, Jürgen (1968): Erkenntnis und Interesse. Frankfurt a.M.: Suhrkamp.

Hacking, Ian (2004): Between Michel Foucault and Erwing Goffman: Between Discourse in the Abstract and Face-to-Face Interaction. In: Economy \& Society 33(3), 277-302.

Hall, Stuart (1996): Introduction: Who needs ,Identity? In: Hall, Stuart \& DuGay, Paul (Hrsg.): Questions of Cultural Identity. London [u.a.]: Sage, 1-17.

Harré, Rom \& Van Langenhove, Luk (Hrsg.) (1999): Positioning Theory. Oxford: Blackwell.

Hermans, Hubert J. M. \& Kempen, Harry (1993): The Dialogical Self. Meaning as Movement. San Diego, CA: Academic Press.

Hermans, Hubert J. M. (2001): The Dialogical Self: Toward a Theory of Personal and Cultural Positioning. In: Culture \& Psychology 7(3), 243-281.

Hilbert, Tatjana S. \& Renkl, Alexander (2007): Concept mapping as a follow-up strategy to learning from texts: What characterizes good and poor mappers? In: Instructional Science 36(1), 36-56.

Holland, Dorothy \& Quinn, Naomi (Hrsg.) (1987): Cultural Models in Language and Thought. Cambridge: Cambridge University Press.

Holzkamp, Klaus (1995): Lernen. Subjektwissenschaftliche Grundlegung. Frankfurt [u.a.]: Campus. 
Hu, Adelheid (1999): Interkulturelles Lernen. Eine Auseinandersetzung mit der Kritik an einem umstrittenen Konzept. In: Zeitschrift für Fremdsprachenforschung, Jg. 10, H. 2, $277-$ 302.

Hu, Adelheid (2005): Kulturwissenschaft(en) und Fremdsprachenforschung - Szenen einer Beziehung. In: Schumann, Adelheid (Hrsg.): Kulturwissenschaften und Fremdsprachendidaktik im Dialog. Perspektiven eines interkulturellen Französischunterrichts. Frankfurt/M: Peter Lang, 45-57.

$\mathrm{Hu}$, Adelheid (2007): Kulturwissenschaftliche Ansätze in der Fremdsprachendidaktik. In: Hallet, Wolfgang \& Nünning, Ansgar (Hrsg.): Neue Ansätze und Konzepte der Literaturund Kulturdidaktik. Trier: WVT, 13-30.

Iberische Lektorenarbeitsgruppe der DAAD-Lektorinnen und -Lektoren (1999): Eine Erhebung zum Deutschlandbild der Germanistikstudentinnen und -studenten auf der iberischen Halbinsel. In: Info DaF 26(4), 355-377.

Jäger, Siegfried (42004): Kritische Diskursanalyse - Eine Einführung. Münster: Unrast-Verlag.

Jørgensen, Marianne W. \& Philipps, Louise (2002): Discourse Analysis as Theory and Method. London [u.a.]: Sage.

Kallenbach, Christiane (1996): Subjektive Theorien. Was Scbüler und Scbülerinnen über Fremdsprachenlernen denken. Tübingen: Narr.

Keller, Reiner (2003): Kultur als Diskursfeld. Deutungsmuster der Abfallpolitik im deutschfranzösischen Vergleich. In: Geideck, Susan \& Liebert, Wolf-Andreas (Hrsg.): Sinnformeln. Linguistische und soziologische Analysen von Leitbildern, Metaphern und anderen kollektiven Orientierungsmustern. Berlin [u.a.]: De Gruyter, 283-305.

Keller, Reiner (2005): Wissenssoziologische Diskursanalyse. Grundlegung eines Forschungsprogramms. Wiesbaden: Verlag für Sozialwissenschaften.

Knapp-Potthoff, Annelie (1997): Interkulturelle Kommunikationsfähigkeit als Lernziel. In: Knapp-Potthoff, Annelie \& Liedke, Martina (Hrsg.): Aspekte interkultureller Kommunikationsfähigkeit. München: Iudicium, 181-205.

Koller, Hans-Christoph (2007): Probleme einer Theorie transformatorischer Bildungsprozesse. In: Koller, Hans-Christoph; Marotzki, Winfried \& Sanders, Olaf (Hrsg.): Bildungsprozesse und Fremdheitserfahrung. Beiträge zu einer Theorie transformatorischer Bildungsprozesse. Bielefeld: Transcript, 69-81.

Koreik, Uwe (2001): Große Töne, wenig Musik. Zur Rolle der Landeskunde im Fach Deutsch als Fremdsprache. In: Aguado, Karin \& Riemer, Claudia (Hrsg.): Zur Theorie, Empirie und Praxis des Deutschen als Fremdsprache (und anderer Fremdsprachen).

Baltmannsweiler: Schneider Verlag Hohengehren, 39-49.

König, Eckhard (1990): Das Konstukt-Interview: Wissenschaftliche Grundlagen, Forschungsmethodik und Probleme. Paderborn (unveröff. Arbeitspapier).

Kramsch, Claire (2000): Social discursive constructions of self in L2 learning. In: Lantolf (Hrsg.), 133-153. 
Kramsch, Claire (Hrsg.) (2002): Language Acquisition and Language Socialization. Ecological Perspectives. London \& New York: Continuum.

Kurtz, Jürgen (2003): Menschenbilder in der Theorie und Praxis des Fremdsprachenunterrichts. Konturen, Funktionen und Konsequenzen für das Lehren und Lernen. In: Zeitschrift für Fremdsprachenforschung 14(1), 149-167.

Lange, Dale L. \& Paige, R. Michael (Hrsg.) (2003): Culture as the Core. Perspectives on Culture in Second Language Learning. Greenwich/Connecticut: Information Age Publishing.

Lantolf, James P. \& Pavlenko, Aneta (1995): Sociocultural Theory and Second Language Acquisition. In: Annual Review of Applied Linguistics 15, 108-124.

Lantolf, James P. (Hrsg.) (2000): Sociocultural Theory and Second Language Learning. New York/Oxford: Oxford University Press.

Lantolf, James P. (2006). Language Emergence: Implications for Applied Linguistics. A Sociocultural Perspective. In: Applied Linguistics 27(4), 717-728.

Lantolf, James P. (2007): Sociocultural Source of Thinking and its Relevance for Second Language Acquisition. In: Bilingualism: Language and Cognition 10(1), 31-33.

Lamnek, Siegfried: ( $\left.{ }^{4} 2005\right)$. Qualitative Sozialforschung. Weinheim [u.a.]: Beltz.

Lave, Jane \& Wenger, Etienne (1991): Situated learning: Legitimate peripheral participation. Cambridge: Cambridge University Press.

Lüders, Jenny (2004): Bildung im Diskurs. Bildungstheoretische Anschlüsse an Michel Foucault. In: Pongratz, Ludwig; Wimmer, Michael; Nieke, Wolfgang; Masschelein, Jan (Hrsg.): Nach Foucault. Diskurs- und machtanalytische Perspektiven der Pädagogik. Wiesbaden: VS.

Norton, Bonny (2000). Identity and Language Learning. Gender, Ethnicity and Educational Change. Harlow: Longman/Pearson.

Meinefeld, Werner ( $\left.{ }^{4} 2007\right)$. Hypothesen und Vorwissen in der qualitativen Sozialforschung. In: Flick, Uwe et al. (Hrsg.): Qualitative Forschung. Ein Handbuch. Reinbek: Rowohlt, 265276.

Ortega, Lourdes \& Iberri-Shea, Gina (2005): Longitudinal Research in Second Language Acquisition: Recent Trends and Future Directions. In: Annual Review of Applied Linguistics 25, 26-45.

Paige, R. Michael et al. (2003a): Culture Learning in Language Education. A Review of the Literature. In: Lange \& Paige (Hrsg.), 173-236.

Paige, R. Michael et al. (2003b). Assessing Intercultural Sensitivity: An Empirical Analysis of the Hammer and Bennett Intercultural Development Inventory. In: International Journal of Intercultural Relations 27(4), 467-486.

Phipps, Alison \& Gonzales, Mike (2004): Modern Languages. Learning and Teaching in an Intercultural Field. London [u.a.]: Sage.

Polkinghorne, Donald E. (1988): Narrative Knowing and the Human Sciences. New York: SUNY Press. 
Rampton, B. (1997): Second Language Research in Late Modernity: A Response to Firth \& Wagner. In: The Modern Language Journal, Jg. 81, H. 3, 329-333.

Reckwitz, Andreas (2000): Die Transformation der Kulturtheorien. Zur Entwicklung eines Theorieprogramms. Weilerswist: Velbrück Wissenschaft.

Rorty, Richard (Hrsg.) (1967): The Linguistic Turn. Essays in Philosophical Method. Chicago: University of Chicago Press.

Sarbin, Theordore R. (1986): The narrative as a root metaphor for psychology. In: Ders. (Hrsg.): Narrative psychology: The storied nature of human conduct. New York: Praeger, 3-21.

Säljö, Roger (1999): Concepts, Cognition and Discourse. From Mental Structures to Discursive Tools. In: Schnotz, Wolfgang et al. (Hrsg.): New Perspectives on Conceptual Change. Amsterdam [u.a.]: Pergamon, 81-90.

Scheele, Brigitte \& Groeben, Norbert (1998): Das Forschungsprogramm Subjektive Theorien. Theoretische und methodologische Grundzüge in ihrer Relevanz für den Fremdsprachenunterricht. In: Fremdsprachen Lebren und Lernen 27, 12-32.

Schmelter, Lars (2004): Selbstgesteuertes oder potenziell expansives Fremdsprachenlernen im Tandem. Tübingen: Narr.

Schmenk, Barbara (2005): Mode, Mythos, Möglichkeiten. oder: Ein Versuch, die Patina des Lernziels ,kommunikative Kompetenz' abzukratzen. In: Zeitschrift für Fremdsprachenforschung 16(1), 57-87.

Schmenk, Barbara (2006): Kraut und Rüben? Kulturwissenschaftliche Ansätze und mögliche Implikationen für die Fremdsprachenforschung. In: Hahn, Angela \& Klippel, Friederike (Hrsg.). Sprachen schaffen Chancen. [...] München [u.a.]: Oldenbourg, 267-278.

Schwerdtfeger, Inge C. (1996): Ansätze für eine anthropologische Begründung der Didaktik des Unterrichts Deutsch als Fremdsprache. In: Info DaF 23, 430-442.

Somers, Margaret R. (1994): The Narrative Constitution of Identity. A Relational and Network Approach. In: Theory and Society 23(5), 605-649.

Spaniel, Dorothea (2002): Methoden zur Erfassung von Deutschland-Images. Ein Beitrag zur Stereotypenforschung. In: Info DaF 29(4), 356-368.

Stäheli, Urs (2000): Poststrukturalistische Soziologien. Bielefeld: transcript.

Steinke, Ines ( $\left.{ }^{4} 2007\right)$ : Gütekriterien qualitativer Forschung. In: Flick, Uwe et al. (Hrsg.): Qualitative Forschung. Ein Handbuch. Reinbek: Rowohlt, 319-331.

Strauss, Anselm \& Corbin, Juliet (1996): Grounded Theory: Grundlagen Qualitativer Sozialforschung. Weinheim: Beltz.

Swain, Merill \& Deters, Ping (2007): ,New` Mainstream SLA Theory: Expanded and Enriched. In: The Modern Language Journal 91(5), 820-836.

Te Molder, Hedwig \& Potter, Jonathan (2005): Conversation and Cognition. Cambridge: Cambridge University Press. 
Ullrich, Carsten (1999): Deutungsmusteranalyse und diskursives Interview: Leitfadenkonstruktion, Interviewfïhrung und Typenbildung. Mannheim: Arbeitspapiere - Mannheimer Zentrum für Europäische Sozialforschung (3).

Watson-Gegeo, Karen A. (2004): Mind, Language, and Epistemology. Toward a Language Socialization Paradigm for SLA. In: The Modern Language Journal 88(3), 331-350.

Weidemann, Doris (2004): Interkulturelles Lernen. Erfahrungen mit dem chinesischen, Gesicht: Deutsche in Taiwan. Bielefeld: Transcript.

Welsch, Wolfgang (1997): Transkulturalität. In: Universitas 52, 16-24.

Wenger, Etienne (1998): Communities of Practice: Learning, Meaning, and Identity. Cambridge: Cambridge University Press.

Wetherell, Margaret (1998): Positioning and Interpretative Repertoires: Conversation Analysis and Post-Structuralism in Dialogue. In: Discourse Society 9(3), 387-412.

Wormer, Jörg (2003): Landeskunde als Wissenschaft. In: Jabrbuch Deutsch als Fremdsprache 29, 435-470.

Zuengler, Jane \& Miller, Elizabeth R. (2006): Cognitive and Sociocultural Perspectives: Two Parallel SLA Worlds? In: TESOL Quarterly 40(1), 35-58.

i Vgl. BMBF-Rahmenprogramm zur Förderung der empirischen Bildungsforschung; November 2007, [www.bmbf.de/pub/foerderung_der_empirischen_bildungsforschung.pdf; 20.09.08].

ii Vgl. im Gegensatz dazu den Überblick von Paige et al. (2003) zu einschlägigen empirischen Forschungsarbeiten im anglophonen Raum.

iii Hier ist v.a. auf Studien aus dem Gießener DFG-Graduiertenkolleg Didaktik des Fremdverstehens zu verweisen (z.B. Brunzel 2002; Bechtel 2003).

iv Als bislang einzig vergleichbare empirische Studie zu individuellen kulturellen Lernprozessen ist die Arbeit von Weidemann (2004) zu nennen, die in Form qualitativer, longitudinal angelegter Fallstudien mit deutschen Muttersprachler/innen in Taiwan jeweils individuelle Alltagstheorien zum ,Gesicht (wahren)' (face) erforscht und auf Grundlage des Forschungsprogramms Subjektive Theorien (FST) (vgl. Groeben et al. 1988) Veränderungen dieser Konstrukte im Laufe sechs- bis zwölfmonatiger Auslandsaufenthalte untersucht.

v Diese Diskussionen weisen nicht nur zentrale Bezüge zu aktuellen gesellschafts- und kulturtheoretischen Diskussionen auf, sie basieren vielmehr - im Sinne der ,Befremdung eigener Sichtweisen` bzw. des kritischen Blicks, von außen` - auf den Erkenntnisständen und Problematisierungsstrategien der (poststrukturalistischen) Anthropologie, Ethnologie, Soziologie und Sozialpsychologie (vgl. expl. Lantolf \& Pavlenko 1995; Rampton 1997); vgl. hierzu auch die ähnlich akzentuierten Plädoyers von Hu (2007) und Schmenk (2006) in Bezug auf die deutschsprachige Diskussion zu ,Kultur' und ,Sprache' in der Fremdsprachendidaktik.

vi Vgl. das Themenheft des Modern Language Journals 91(5) zur cognitive-social debate.

vii Zu diesem Ergebnis kommen auch andere Vertreter des Faches und leiten daraus den Bedarf an einer wissenschaftlichen Fundierung der Landeskunde ab (vgl. z.B. Koreik 2001, Wormer 2003).

viii Im Sinne Lamneks als „Bindeglied zwischen Theorie und Empirie“ (vgl. 2005: 132).

ix Vgl. hierzu Habermas (1969: 155ff.).

x Im Sinne des Kapitalsortenansatzes von Bourdieu (z.B. 1983: 183ff). 
xi Zu operativen Begriffen vgl. Welsch (1997): „Kulturbegriffe sind immer mehr als bloß beschreibende Begriffe, sie sind operative Begriffe. Wie andere Selbstverständigungsbegriffe auch (beispielsweise Identität, Person, Mensch), haben sie stets Einfluß auf ihren Gegenstand, verändern diesen. [...] In diesem Sinn ist die ,Realität von Kultur immer auch eine Folge unserer Konzepte von Kultur" (20).

xii „Wenn wir lernen, zwischen der prinzipiellen methodischen Offenheit und der Expliziertheit, mit der das Vorwissen reflektiert und ausformuliert wird, zu unterscheiden, wird es möglich, die Formulierung von Hypothesen mit dem Rekonstruieren gegenstandsspezifischer Bedeutungsgehalte zu vereinbaren“ (Meinefeld 2007: 272).

xiii Das Forschungsprogramm von Keller integriert wissenssoziologische und diskurstheoretische Analytiken, versteht Deutungsmusteranalyse als eine mögliche analytische Perspektive auf ,Diskurse und wird im Rahmen des Dissertationsprojekts in einer empirischen Teilstudie ebenfalls in modifizierter Form angewendet.

xiv „Kultur als Diskursfeld zu begreifen, impliziert, auf Auseinandersetzungen um Reproduktion und Veränderung von Deutungsweisen und Handlungspraktiken hinzuweisen, die vielgestaltige Strukturiertheit, (Re-)Produktion, Heterogenität und Wandelbarkeit soziokultureller [...] ,Vielheiten [hervorzuheben], die Bedeutung der diskursiven Artikulationskämpfe für die Erzeugung, Identitätsstabilisierung und Transformation solcher Diskurs-Kollektive zu betonen“ (Keller 2003: 285).

xv In welchem Verhältnis diese Sicht zu neueren deutschsprachigen Arbeiten im Feld der Gouvernementalitätsforschung bzw. zur ,Genealogie der Subjektivierung' steht, ist noch zu prüfen. Beispielhaft sei hier auf eine Studie von Bröckling (2007) verwiesen, die am Beispiel des ,unternehmerischen Selbst' ein „Bündel aus Deutungsschemata [analysiert], mit denen Menschen sich selbst und ihre Existenzweisen verstehen, aus normativen Anforderungen und Rollenangeboten, an denen sie ihr Tun und Lassen orientieren, sowie aus institutionellen Arrangements, Sozial- und Selbsttechnologien, die und mit denen sie ihr Verhalten regulieren sollen“ (7).

xvi Im mehr oder minder direkten Anschluss an Groeben et al. (1988) stehen dabei Subjektive Theorien (als relativ stabile kognitive Strukturen) von Fremdsprachenlehrenden im Zentrum des Forschungsinteresses, während subjektiven Lernertheorien, insbesondere aber deren Veränderungen, erst in neuerer Zeit verstärkt Aufmerksamkeit geschenkt wird (vgl. jedoch bereits Kallenbach 1996).

xvii Vgl. auch Breidbach 2007 mit einer vergleichbaren, m.E. äußerst fruchtbaren und v.a. ausformulierten Argumentationslinie für den Bereich des bilingualen Sachfachunterrichts.

xviii Aus forschungspraktischer Perspektive wird ein 'Diskurs' als ein „virtuelles Textkorpus“ verstanden, das als solches der Analyse nicht zugänglich und von einem „konkreten Textkorpus“ zu unterscheiden ist (vgl. Busse \& Teubert 1994).

xix Vgl. zur Strukturierung des Korpus auch Altmayer (2007). Das hier nicht näher vorzustellende diskursanalytische Vorgehen orientiert sich an forschungspragmatisch ausgerichteten Analytiken, v.a. jenen von Jäger (2004), Keller (2005), Altmayer (2007). 



\title{
Das Europäische Sprachenportfolio zwischen Traum und Wirklichkeit
}

Eine kritische Betrachtung dieses Instruments aus der Sicht italienischer LehrerInnen

\author{
Sabine C. Stricker (Trento)
}

\begin{abstract}
Das Europäische Sprachenportfolio (ESP) wurde laut Schulreform 2003 in Italiens Schulen eingeführt. Die folgende Arbeit möchte das ESP aus der Perspektive italienischer DaF-LehrerInnen betrachten; es sollen so „Traum und Wirklichkeit“ gegenübergestellt werden: „Traum“ im Sinne der theoretischen Konzepte und Ziele des ESP. „Wirklichkeit“ in Form der Erwartungen, Bedenken und Fragen aus der Sicht der Lehrenden.
\end{abstract}

Ich sehe noch einen langen Weg bis zum Ziel.i

\section{Einleitung}

Europa vereint viele verschiedene Kulturen und ebenso viele Sprachen. Dieser Pluralismus und diese Vielfältigkeit wurden vom Europarat als Charakteristikum Europas anerkannt und soll geschützt und erhalten werden (Charta der Grundrechte Nizza 2000). Die europäische Sprachenpolitik will/muss Mehrsprachigkeit fördern und fordern: die zukünftigen BürgerInnen Europas sollen zwei europäische Sprachen über die Erstsprache hinaus beherrschen (Weißbuch 1998; Brüll 1999; Kommission der europäischen Gemeinschaften 2003). Um dieses Ziel zu erreichen, hat der Europarat verschiedene Initiativen ins Leben gerufen, unter anderem den Gemeinsamen europäischen Referenzrahmen für Sprachen (GER) und das Europäische Sprachenportfolio (ESP).

Ziel der hier vorgestellten Qualifikationsarbeit ist es, den Einsatz des ESP aus unterschiedlichen Perspektiven zu betrachten und seine Auswirkungen auf den Alltag des Sprachenlernens zu untersuchen.

Das ESP aus der Sicht der Lehrenden: In drei Fokusgruppen wurden verschiedene Aspekte der Arbeit mit dem Sprachenportfolio diskutiert, aber vor allem Zweifel und Befürchtungen der Lehrpersonen festgehalten.

Das ESP aus der Sicht der Lernenden: In drei Projekten (GermanistikstudentInnen der Universität Trient und zwei Schulklassen Trentiner Gymnasien) wurde mit dem ESP gearbeitet. Durch Interviews in Form von Fokusgruppen, Fragebögen, Beobachtungen der LehrerInnen und eine Analyse der verschiedenen erstell- 
ten Sprachenportfolios wurde versucht, ein möglichst aussagekräftiges Bild der Implementierung des ESP im Trentiner Lernalltag zu zeichnen.

Im folgenden Beitrag möchte ich neben allgemeinen Aspekten des ESP auf die Resultate der Fokusgruppe der Lehrpersonen eingehen, es sollen also Traum und Wirklichkeit gegenübergestellt werden: Traum - im Sinne der theoretischen Konzepte und Ziele des ESP; Wirklichkeit - in Form der Reflexionen einer Gruppe von FremdsprachenlehrerInnen der Provinz Trient in Norditalien.

\section{Ziele des ESP}

The ELP project has two main aims: a) to motivate learners by acknowledging their efforts to extend and diversify their language skills at all levels; b) to provide a record of the linguistic and cultural skills they have acquired (to be consulted, for example, when they are moving to a higher learning level or seeking employment at home or abroad) (Schneider \& Lenz 2001: 3).

Die Ziele des ESP gehen Hand in Hand mit den Zielen der europäischen Sprachenpolitik (Council of Europe 1998; Schneider \& Lenz 2001; Ministero dell'Istruzione, dell'Università e della Ricerca 2005) und können mit folgenden Schlagworten zusammengefasst werden: Mehrsprachigkeit, Mobilität, Autonomes Lernen, Transparenz und erweiterte Leistungsbeurteilung.

Um Mehrsprachigkeit zu fördern, muss das Fremdsprachenlernen zu einem lifelong-learning werden, das soll heißen, dass das Lernen einer Fremdsprache nicht nur in einem schulischen Kontext zu sehen ist, sondern als etwas, das in jedem Lebensabschnitt unabhängig von institutionellen Kursen und Ausbildungen stattfindet. Ein vereintes Europa heißt auch ein vereinter Arbeitsmarkt, der von den ArbeitnehmerInnen eine gewisse Flexibilität fordert. Flexibilität nicht nur im Sinne eines Wohnsitzwechsels, sondern auch im Sinne der Aneignung einer neuen Sprache aus professionellen Gründen. Das Ziel wäre, Menschen dahingehend auszubilden, dass Fremdsprachenlernen im Schulbereich auch die Basis für weiteres außerschulisches Sprachenlernen schafft. Eines der wichtigsten Anliegen des ESP ist eine Veränderung unserer Haltung zum Sprachenlernen: Das Lernen einer neuen Sprache soll für Erwachsene nicht mehr als etwas Außergewöhnliches, sondern als machbare Herausforderung, für die man in seiner Schulausbildung gut genug vorbereitet wurde. Die Arbeit mit dem ESP erfordert autonomes Lernen: die schulische Sprachausbildung sollte demnach so organisiert werden, dass die Lernenden nicht lediglich eine neue Sprache lernen, sondern durch dieses Lernen auch die Fähigkeit erwerben, späteres autonomes Sprachlernen kompetent anzugehen.

Ein weiteres Ziel des ESP ist Transparenz (Schneider 1999; Parlamento europeo 2004): Welche Französischkompetenzen können von einer Person erwartet werden, die in Österreich ein „befriedigend“ im Maturazeugnis hat? Hat sie die gleichen Kompetenzen wie eine Schülerin aus dem Aostatal in Norditalien mit einem „buono“ in ihrem Abschlusszeugnis? - Wohl kaum. Kann man in Schweden 
mit diesen Informationen etwas anfangen? Wie können Schulnoten Aussagekraft haben, wenn man nichts über den Schultyp, das Curriculum, die Methode und die verwendeten Lehrwerke weiß? Um Mobilität erst möglich zu machen, muss zunächst Transparenz garantiert werden. Transparenz im Sinne einer gemeinsamen Sprache zur Beschreibung von Sprachkompetenzen, die in der Schulausbildung, aber auch außerhalb, erworben wurden. Diese gemeinsame Metasprache soll durch den Gemeinsamen Europäischen Referenzrahmen (GER) garantiert werden. Alle weiteren Daten zu Schultyp und Lehrplan können durch das ESP gegeben werden, mehr noch, das ESP bietet die Möglichkeit auch außerschulisch erworbene Kompetenzen zu dokumentieren und damit ein detailreiches, buntes Bild erworbener Kompetenzen zu zeichnen und potentiellen ArbeitgeberInnen in allen europäischen Mitgliedstaaten transparente, d.h. verständlich und vergleichbar Informationen vorzulegen.

Damit kommen wir zum letzten Ziel des ESP: Der erweiterten Leistungsbeurteilung (Schneider 1999). Das ESP will eine Plattform sein, um auch außerschulisch erworbene Kompetenzen zu dokumentieren, einerseits zur Bewusstmachung, wo überall im Alltag Fremdsprachenlernen stattfinden kann, andererseits zur Erweiterung der Informationen über erworbene Kenntnisse, indem z.B. schulische Leistungsbeurteilung mit Selbsteinschätzung gekoppelt wird. Wird zum Beispiel in einer spezifischen Sprachausbildung viel Wert auf schriftliche Kompetenzen in einer Fachsprache gelegt, die betroffene Person allerdings auch nennenswerte Kompetenzen im mündlichen Bereich zu bieten hat, so gibt das ESP Platz für diese wichtigen Zusatzinformationen.

\section{Was ist nun das ESP?}

Das ESP ist eine Sammlung von Dokumenten unterschiedlicher Art (Zeugnisse, Teilnahmebestätigungen, u. ä.) und Beispielen persönlicher Arbeiten, die von den Lernenden zusammengestellt werden und die sie immer wieder ergänzen und aktualisieren (Little \& Perclova 2000; Schneider \& Lenz 2001).

Das ESP besteht aus drei Teilen: dem Sprachenpass, der Sprachbiographie, auch Sprachlernbiographie genannt, und dem Dossier.

\subsection{Der Sprachenpass}

Der Sprachenpass zeigt, welches Niveau in den verschiedenen Fremdsprachen erreicht wurde, es dokumentiert die Resultate von Sprachlernen, aber auch interkulturellen Erfahrungen. Im Sprachenpass sollen neben den globalen Selbsteinschätzungen der Sprachkompetenzen Zeugnisse, Diplome und Bestätigungen über besuchte Sprachkurse und andere Sprachlernerfahrungen (Auslandsaufenthalte, Tandemkurse) gesammelt werden. 
Der Sprachenpass ist für alle Modelle identisch: ein DinA5-Heft, dessen graphische Gestaltung nicht besonders gut gelungen ist und das Verständnis keineswegs erleichtert. Es lassen sich z. B. die zwei Abschnitte „Überblick über Sprachlernen und interkulturelle Erfahrungen“ im eigenen Land und im Land der Zielsprache nur durch ganz genaues Hinsehen unterscheiden und die vorgesehenen Tabellen stiften auch beim Ausfüllen Verwirrung.

\subsection{Die Sprachbiographie}

Die Sprachbiografie lädt zur Reflexion ein: Ein Nachdenken über den eigenen sprachlichen Hintergrund und die Geschichte des individuellen Sprachenlernens, den besuchten Sprachunterricht, dessen Methoden, Lehrwerke und Lernziele; und ein Nachdenken über wichtige interkulturelle Erfahrungen, individuelle Lernziele und Schwerpunkte, über Lernstile und -strategien, über das „Wie kann man Sprachen lernen?“ im Vergleich zum „Wie lerne ich Sprachen?“

Die Sprachbiographie ist sozusagen die Arbeitsplattform, wo man detailliert Kompetenzen einschätzt, eigene Arbeiten beurteilt, autonomes Lernen plant und über individuelle Lernstrategien nachdenkt, wo man sich mit dem Prozess des Sprachlernens auseinandersetzt, während im Sprachenpass lediglich die Fakten und Resultate festgehalten und präsentiert werden.

Abgesehen von bestimmten Schwerpunkten finden wir aber in allen Model$\operatorname{len}^{\text {ii }}$, in unterschiedlicher Ausarbeitung und Intensität, Fragen zum sprachlichen Hintergrund. Es zählt nicht nur mit welchen Sprachen und Dialekten man aufwächst, sondern auch neben welchen Sprachen man gelebt hat. Interessant finde ich die Aufwertung der Dialekte: in manchen Modellen iii fragt man gezielt nach Erfahrungen mit und Kompetenzen in Dialekten.

Die verschiedenen Modelle des ESP sind auf ein konkretes Zielpublikum zugeschnitten, was sich sowohl in den detaillierten Kannbeschreibungen im Sinne einer unterschiedlichen Gewichtung auf bestimmte Lebensbereiche, Kompetenzen $^{\text {iv }}$ oder Fachsprachen ${ }^{v}$ als auch in Arbeitsvorschlägen zur autonomen Lernpla-

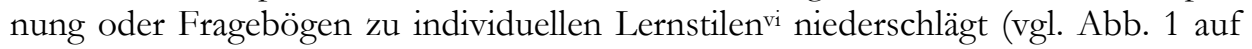
der folgenden Seite).

\subsection{Das Dossier}

Das Dossier enthält eine Auswahl von persönlichen Arbeiten, die zeigen sollen, welche Kompetenzen man in den verschiedenen Sprachen erreicht hat. Es kann sich um verschiedene Materialien handeln, sowohl schriftliche Arbeiten wie auch Audio- und Videoaufnahmen, aber auch Ergebnisse von Projektarbeiten.

Die Auswahlkriterien können unterschiedlich sein: Zeigt man Fertigkeiten und Kompetenzen so kommt das Endprodukt in Frage, will man hingegen den Lernprozess, also wichtige Lernschritte, dokumentieren, so kann man auch unkorrigierte Arbeiten aufnehmen. Generell entscheidet der/die BesitzerIn des ESP. Die 
Auswahl der Arbeiten für das Dossier wird aber auch durch die potentiellen LeserInnen $^{\text {vii }}$ des ESP beeinflusst. In einer Bewerbung für eine Arbeitstelle sind Produkte zu bestimmten Themen und Fertigkeiten von Interesse, während für einen Stipendienantrag auch Lernprozesse interessant sein können.

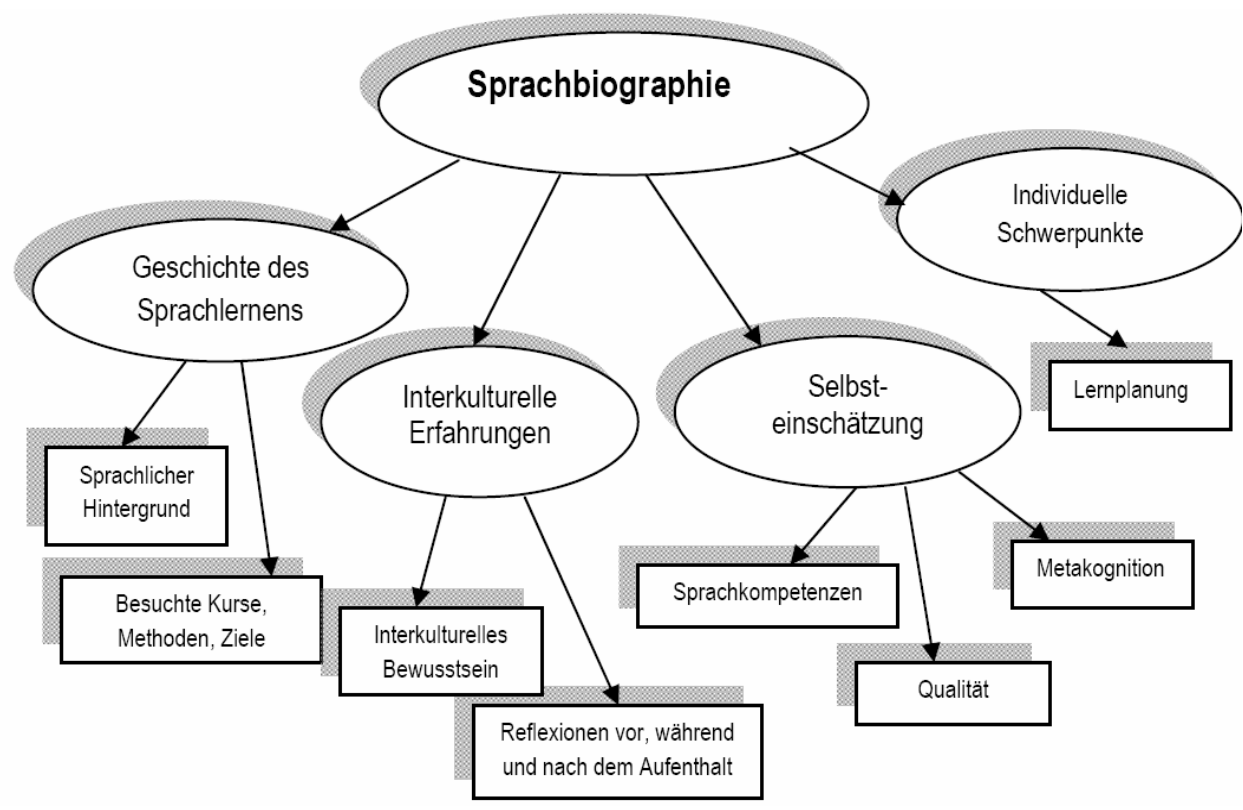

Abb. 1: Die Sprachbiographie.

\section{Die Funktionen des ESP}

Das ESP hat zwei Funktionen: eine dokumentative und eine pädagogische. In seiner dokumentativen Funktion ist es einem Ausstellungs- und Evaluationsportfolio sehr ähnlich. Das Produkt, das Resultat steht im Mittelpunkt, im Sinne einer Inventur am Ende eines Studienganges, auf der Suche nach einer Arbeitsstelle oder bei der Bewerbung für ein Stipendium.

In seiner pädagogischen Funktion ist das ESP mit einem Entwicklungs- oder Sollportfolio vergleichbar und will Hilfsinstrument für autonomes, individuelles Lernen sein. 


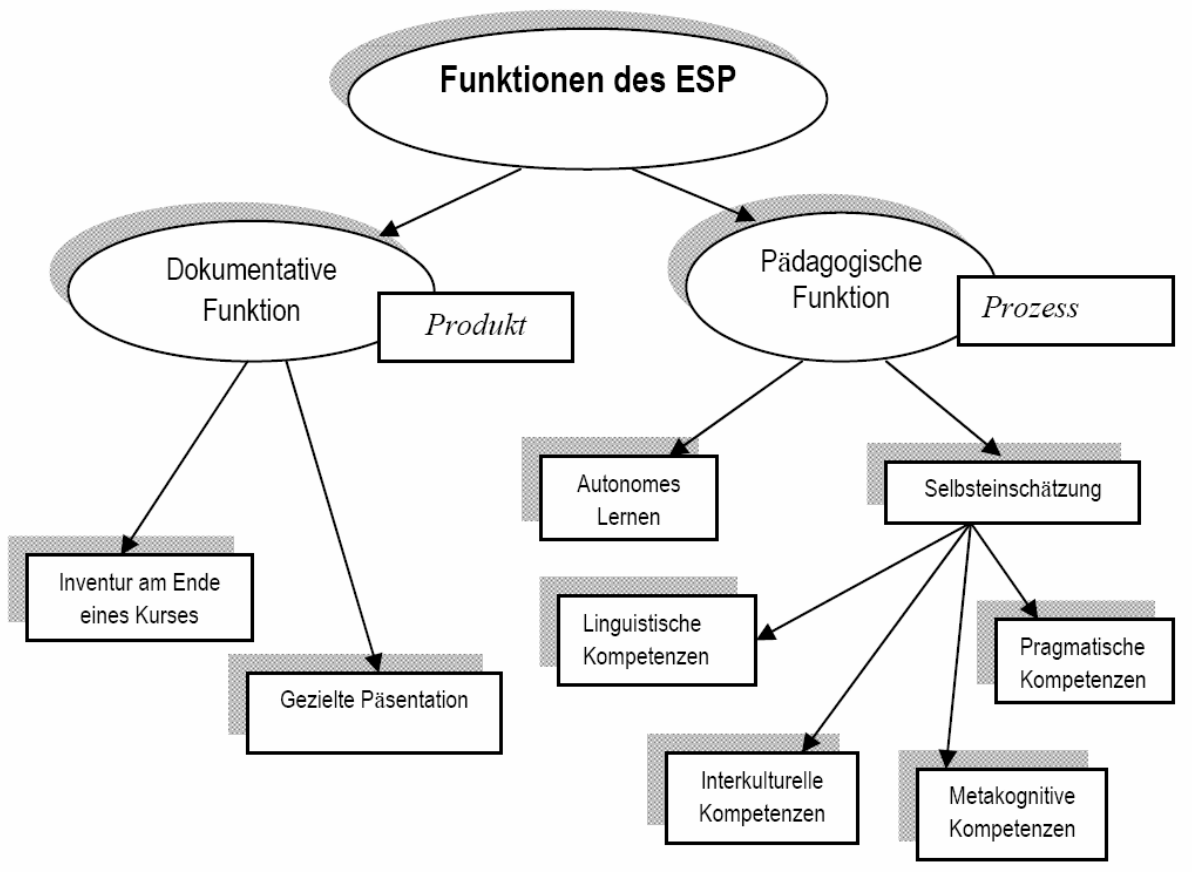

Abb. 2: Die Funktionen des ESP.

\section{Das ESP aus der Sicht der Lehrenden}

Ich finde die Idee des Portfolio gut, weil ich glaube, dass es ein wertvolles Instrument ist, da es ,international' und praxisorientiert ist.*viii

Für das ESP wurde in den Jahren 2002/2003 in Italien viel Werbung gemacht, das Goethe-Institut widmete eine Ausgabe der Zeitschrift PerVoi diesem Thema, Erfolgsberichte der ersten Pilotversuche sowohl auf nationaler (Billoto 2000; Palamidesi 2002; Ufficio Scolastico Regionale per il Piemonte del MIUR 2003) als auch auf europäischer Ebene (Schärer 2000) wurden vermittelt und die Schulreform von 2003 nahm diese Idee sogleich auf. Man setzte hohe Erwartungen in dieses Instrument.

Für Italien wurden zwischen 2002 und 2008 elf ESP-Modelle von der zuständigen Kommission des Europarates akkreditiert: 


\begin{tabular}{|l|l|l|l|l|l|l|l|l|l|l|l|l|l|l|}
\hline $\begin{array}{l}\text { Alter I } \\
\text { Modell }\end{array}$ & 7 & 8 & 9 & 10 & 11 & 12 & 13 & 14 & 15 & 16 & 17 & 18 & 19 & 20 \\
\hline 25.2002 & & & & & & & & & & & & & & \\
\hline 26.2002 & & & & & & & & & & & & & & \\
\hline 30.2002 & & & & & & & & & & & & & & \\
\hline 40.2003 & & & & & & & & & & & & & \\
\hline 48.2003 & & & & & & & & & & & & & & \\
\hline 49.2003 & & & & & & & & & & & & & & \\
\hline 54.2003 & & & & & & & & & & & & & & \\
\hline 64.2004 & & & & & & & & & & & & & & \\
\hline 65.2004 & & & & & & & & & & & & & \\
\hline 69.2004 & & & & & & & & & & & & & \\
\hline
\end{tabular}

Abb. 3: Italienische ESP-Modelle.

Alle validierten Modelle wurden in einem Pilotprojekt getestet und die Resultate sind bei allen als sehr positiv zu bewerten.

Generell stehen die Lehrkräfte diesem Instrument aber deutlich kritischer gegenüber als man sich durch die Lektüre der verschiedenen Berichte und Analysen erwarten würde (Thürmann 2007). Allem voran sollte unterstrichen werden, dass alle Projekte von den AutorInnen (zuständige Schulämter der jeweiligen Region) der ESP-Modelle durchgeführt wurden, was die Sichtweise beeinflussen mag. Zur Erklärung der durchgehend positiven Resultate dient wohl auch der Zeitpunkt der Forschungsprojekte, die alle zwischen 1998 und 2004 (Bilotto 2000; Canulli \& Pedrazzini 2003; Ufficio Scolastico Regionale per il Piemonte del Miur 2003; Civegna et al. 2007) durchgeführt wurden und die Desillusionierungsphase erst später eintritt, wenn die Pilotprojekte abgeschlossen sind und weitere Finanzierungen ausbleiben; weiters wird Misserfolg selten dokumentiert.

Im Folgenden werde ich auf die Resultate einer Fokusgruppe (Ghislandi 2004) mit Trentiner Fremdsprachenlehrerinnen ${ }^{\text {ix }}$ verschiedener Gymnasien eingehen und komme, nachdem ich im ersten Teil den „Traum“ des ESP beschrieben habe, zur mitunter etwas ernüchternden Wirklichkeit.

Ziel der Fokusgruppe (April und Oktober 2005) mit Lehrerinnen ist, eine Art Inventur der Eindrücke und Bedenken, aber auch ihrer Erwartungen zu machen. Die Form der Fokusgruppe, also einer halbstrukturierten Gruppendiskussion, erscheint die beste Art zu sein, Meinungen und die dahinter liegenden Motive zu sammeln. Die Fokusgruppe als Instrument qualitativer Forschungen erlaubt in einer Problemstellung in die Tiefe zu gehen, wo z. B. Fragebögen nicht in der gleichen Weise einzusetzen wären. Die Teilnehmerinnen haben hier die Gelegenheit, ihre Meinungen auszudrücken bzw. im Dialog mit anderen Lehrerinnen Ideen, Hypothesen und Eindrücke zu formulieren. Nachteil der Methode dieser Grup- 
pengespräche ist allerdings die Unvorhersagbarkeit des Gesprächsverlaufs, d.h. es können verschiedene Schwerpunkte vorbereitet werden, doch entscheidet am Ende die Gruppe den Gesprächsverlauf. Die Resultate einer solchen Untersuchung können wegen der geringen Zahl der Teilnehmerinnen nicht als repräsentativ angesehen werden, doch sind sie in unserem Falle eine interessante Bereicherung und sichern eine andere Sichtweise auf ein und das selbe Phänomen.

\subsection{Die Rolle der Lehrenden}

Wie sehr sollen wir präsent sein? Was ist unsere Rolle?*

Das ESP ist wie bereits erwähnt Eigentum seiner BesitzerInnen, doch kann diese Freiwilligkeit bei jungen Lernenden nicht absolut gesetzt werden, was eine mehr oder weniger starke Einbindung in den Unterricht notwendig macht. Hinzu kommt, dass unsere SchülerInnen wenig Erfahrung mit autonomem Arbeiten ${ }^{\mathrm{x}}$ haben und die Arbeit mit dem ESP für alle neu ist. Die Teilnehmerinnen der Fokusgruppe fragen sich alle, wie sehr sie in der Portfolioarbeit präsent sein sollten, was ihre Rolle sei.

Ein Großteil der Gruppe meint sogar, es sei Aufgabe der Lehrerinnen das ESP für ihre SchülerInnen zu gestalten. Dieses Missverständnis lässt sich dadurch erklären, dass die Portfolioidee im Kompetenzportfolio der Schulreform Moratti xi eine gänzlich verschiedene Interpretation gefunden hat. Hier ist es tatsächlich Aufgabe der Lehrpersonen, ein Kompetenzportfolio für die SchülerInnen zu erstellen. Aus dieser Perspektive wird verständlich, warum die Einführung des ESP von Seiten der LehrerInnen mitunter negative Reaktionen auslöste.

Dennoch bleibt die Frage nach der Rolle des ESP in der Arbeit mit der Klasse und inwieweit die Lehrperson die Arbeit mit dem ESP lenken soll/darf, noch offen. Es scheint Unsicherheit zu schaffen, die Entscheidungen, welche Arbeiten ins Dossier aufgenommen und gezeigt werden, allein den einzelnen Lernenden zu überlassen. Mehr noch: Diesen die Freiheit zu lassen, ob ein Text korrigiert oder unkorrigiert ins Dossier gegeben werden soll, ist für viele Lehrende nahezu undenkbar. Generell fällt es den Beteiligten nicht leicht, den Lernenden Autonomie zuzutrauen, was - wenn auch nur unbewusst und indirekt präsent - jede Entwicklung in Richtung Autonomie erschwert. Eine Interpretation dieser Kontroverse kann auch vorauseilender Gehorsam sein: die Lehrperson sieht ihre Rolle nicht in der Gestaltung einer Lernsituation, sondern als Lehrende, also aktiver und damit verantwortlicher Part.

Im Arbeitsdossier, da ist die Unterstützung der Lehrerin notwendig, da sie ja helfen sollte, sich zu verbessern. Wer eine Arbeit gemacht hat, die der Lehrerin gezeigt wird, um sie ins Dossier zu geben, sollte kommentiert werden, ob das gut ist oder nicht, es braucht die Korrektur der Lehrerin.* 
Die Lehrperson fühlt sich verpflichtet, wie das Zitat klar zeigt, die Produkte, die ein/e SchülerIn ins Dossier geben möchte, abzusegnen, dies spiegelt ganz klar die Diskrepanz zwischen Autonomie, Selbstverantwortung und gängiger Lern- und Lehrkultur wider. Luciano Mariani (2002: 13) spricht von den verschiedenen sfidexii, die die Arbeit mit dem Sprachenportfolio für die Fremdsprachendidaktik darstellt. Ist der/die SchülerIn Besitzerin des ESP, so sollte die Motivation von ihr/ihm kommen, was im Schulbereich zu den zu erwartenden Schwierigkeiten führt. Mariani betont auch die Konsequenzen dieses „Besitzens“, da Kompetenzen dokumentiert werden sollten, die für den Lernenden relevant und wichtig sind, was nicht unbedingt mit dem Lehrplan übereinstimmen muss.

Darüber hinaus unterstreicht Mariani richtigerweise, dass ein ESP, sollte es wirklich ein persönliches und individuelles Instrument sein, nicht nur institutionell erwartete Kompetenzen dokumentieren soll, sondern auch unerwarteten Resultaten Raum geben muss.

\subsection{Der Faktor Zeit}

Der Faktor Zeit ist für mich der heikelste Punkt, wie viel Zeit investiere ich?*

Die größten Bedenken schafft der Faktor ,Zeit“", man fragt sich, wie viel Zeit sollte/muss investiert werden. Die Angst wertvolle Unterrichtsstunden zu verlieren, wird von allen Teilnehmerinnen geteilt, da das vorgesehene Programm ohnehin schon als intensiv beschrieben wird und für neue Projekte demnach kaum Platz bleibt. Das bestätigt die bereits 2002 von Keller (2002: 20) formulierten Bedenken: „Die Arbeit mit dem Portfolio erfordert mehr Zeit als generell zur Verfügung steht und die LehrerInnen sind oft nicht bereit (oder trauen sich auch nicht) Schnitte und Streichungen an den geplanten und vorprogrammierten Unterrichtsaktivitäten vorzunehmen."

Auch stehen die Lehrerinnen, was das folgende Zitat gut wiedergibt, unter einem gewissen Leistungsdruck. Werden innovative Methoden eingesetzt, sollten möglichst schnell Resultate zu sehen sein, was allerdings bei pädagogischen Lernzielen wie Autonomie nicht leicht zu erwarten ist.

Natürlich haben wir viele Zweifel, unsere Angst ist, dass es sehr 'technisch' ist, dass man viel Zeit verliert und nichts rauskommt.*

Generell habe ich den Eindruck, dass jede Art von innovativem Arbeiten vor dem Kollegium gerechtfertigt und „verteidigt" werden muss, was diesen spürbaren Leistungsdruck erklären kann und auch durch die folgende Aussage unterstrichen wird.

Vielleicht ist ein Grund für die Bedenken mit dem Portfolio zu arbeiten, dass dadurch schwarz auf weiß auch unsere Arbeit dokumentiert wird.* 
Transparenz kann auch das Problem sein: in der Portfolioarbeit beurteilt nicht nur der/die Lehrende, sondern die Arbeit im Unterricht wird dokumentiert, um sie vorzeigen zu können, also auch von Dritten (KollegInnen und Außenstehenden) bewerten zu lassen.

Die StudentInnen des ESP-Projekts der Universität geben auch an, dass ihnen z. B. die Dokumentation und Lernplanung zu zeitaufwendig erscheint; hier sollte man sich überlegen, wie dies im Unterricht integriert werden kann, ohne dass sowohl Lehrende als auch Lernende das Gefühl haben, wertvolle Zeit zu verlieren.

\subsection{Die Dokumentationsfunktion}

Trotz aller Zweifel erwartet man sich aber von diesem Instrument, eine Brücke zwischen den verschiedenen Schulen zu sein; ein Mittel, um Kontinuität im Sprachenlernen zu garantieren. Jeder Übergang von einem Bildungszyklus zu einem anderen sollte einen Neuanfang bedeuten. Oft jedoch werden bereits gemachte Erfahrungen nicht in Betracht gezogen. Diese mangelnde Kommunikation zwischen den verschiedenen Bildungszyklen wurde in unserer Fokusgruppe intensiv diskutiert: Man erwartet sich im ESP eine Möglichkeit der Kommunikation von Lernerfahrungen und erworbenen Kompetenzen zu finden.

[...], dass sie (die SchülerInnen Anm. d.A.) ein 'Gedächtnis' haben könnten, von all dem, was sie im Laufe der Jahre gemacht haben, ihnen Resultate in die Hand zu geben, einen Beweis, das wäre, glaube ich, sehr motivierend für sie, die eigenen Fortschritte zu sehen.*

Die Ergebnisse einer Analyse von 45 Sprachbiographien ${ }^{x i i i}$ sind eher ernüchternd, weil keine zusätzlichen Informationen über den Lernprozess/die Lerngeschichte vermittelt wird, was mit der Unerfahrenheit der Lernenden mit Portfolioarbeit, mit Selbsteinschätzung und Lernplanung zu erklären ist. Tatsache bleibt jedoch, dass man mit dieser Analyse (abgesehen von einigen Aspekten) nicht viel mehr erfährt, als man bereits weiß. Je mehr Erfahrungen allerdings sowohl die LehrerInnen als auch die SchülerInnen mit diesem Lernbegleiter sammeln, desto eher kann man sich erwarten, durch die Lektüre eines ESP fundierte Informationen zu bekommen. Die Gruppe zweifelt jedoch nicht daran, dass diese Dokumentation des Lernens und das Sichtbarmachen von Lernerfolg die Motivation der LernerInnen nährt und Mut gibt, Neues auszuprobieren.

Ich finde es wichtig, dass sie (die SchülerInnen, Anm. d.A.) verstehen, was sie gelernt haben. Denn wenn du einen 15-Jährigen fragst, der 10 Jahre Deutschunterricht hatte, was er kann, dann kommt ,Nichts' als Antwort, das ist jedoch nicht richtig, denn wenn sie kommunizieren müssen, wissen sie einiges, aber sie neigen dazu, sich zu unterschätzen.* 


\subsection{Der metakognitive Aspekt des ESP}

Das ESP will in seiner pädagogischen Funktion zum Nachdenken über das eigene Lernen einladen, doch kann man auch hier sowohl aus der Sicht der Lernenden als auch aus der Sicht der Lehrenden nicht von Anfang an Erfolge erwarten. Im ESPProjekt mit den StudentInnen der Universität Trient ist z. B. dieser Aspekt der eindeutig schwächste: Die Evaluation des Arbeitens und Lernens, unabhängig von der Selbsteinschätzung sprachlicher Kompetenzen bzw. Leistungen, wird gerne vergessen, erst im Gespräch in der Gruppe finden tatsächlich Reflexionen und Diskussionen über Lernstrategien statt.

Ich finde, auch wenn man auf italienisch über das Lernen reflektiert, hilft es, die Sprache und das Lernen zu verstehen. Das ist für mich gut investierte Zeit.*

Diskussionspunkt in der Fokusgruppe ist die Frage, ob diese Reflexionen in der Erstsprache oder in der Fremdsprache geführt werden sollten. Bei niedrigeren Kompetenzniveaus ist das unvermeidbar. Vorteil bei der Reflexion in der Erstsprache ist, dass der fächerübergreifende Charakter des ESP wichtig wird und die Zusammenarbeit bzw. verstärkte Kommunikation unter den KollegInnen der anderen Sprachen fördert.

Prinzipiell ist sich die Fokusgruppe einig, dass die Einführung des ESP vom gesamten Kollegium getragen werden sollte, da es erst so effizientes Arbeiten erlaubt, sein transversaler Charakter genutzt wird, aber auch Mut zum Experimentieren gibt.

\subsection{Erfahrungen mit Selbsteinschätzung}

Meinen SchülerInnen fällt es schwer, eigene Fähigkeiten zu erkennen, das geschieht nicht so direkt.*

Sind unsere SchülerInnen fähig, sich selbst einzuschätzen?, ist eine intensiv diskutierte Frage. Man fragt sich, wie man Selbsteinschätzung üben kann.

Für die im Projekt involvierten Trentiner StudentInnen ist die Arbeit mit Selbsteinschätzungxiv völlig neu, was ihnen aber noch mehr Schwierigkeiten bereitete, war die Selbsteinschätzung von Kompetenzen in Form von Kannbeschreibungen und nicht im Sinne einer Beurteilung durch eine Note. Dies wird von der Fokusgruppe bestätigt:

Ja, ich sehe das wie ein zweischneidiges Schwert, einerseits sehr positiv, ich gebe dem Lernprozess mehr Gewicht, aber manchmal verunsichert das, vielleicht weil wir in unserer Kultur gewohnt sind, genau zu quantifizieren und das kann desorientieren. Sich selbst einschätzen zu können, ist ein Prozess der Zeit braucht, man nähert sich dem Schritt für Schritt und dann wären die Noten überholt, dann ging's auch ohne. Aber bis dahin ist es noch weit.* 
Interessant ist auch zu beobachten, dass sowohl die Lernenden als auch die Lehrenden in der Kombination Selbsteinschätzung-Fremdeinschätzung Halt suchen, was aber auch ein Schwachpunkt werden kann. Koppelt man Selbsteinschätzung immer an Fremdeinschätzung, kann dies sehr wohl helfen, Vertrauen in eigene Evaluation zu gewinnen. Es besteht aber auch die Gefahr, dass versucht wird, möglichst nahe an die Resultate der Lehrperson heranzukommen und weniger die individuelle Sicht der eigenen Kompetenzen zu eruieren. Selbsteinschätzungen stellen die subjektive Sicht des Lernenden dar und sollen als solche stehen bleiben dürfen.

Wie schwer es den Lernenden fällt mit den Kannbeschreibungen zu arbeiten, ist auch ein interessanter Punkt im Gespräch der Fokusgruppe:

Die erste Frage der Jugendlichen war: 'Was heißt das? Was ist damit gemeint?'

Die Terminologie war für Schüler nicht passend.*

Ein Kritikpunkt von vielen ESP-Modellen ist, dass die Deskriptoren (Keller 2002) sich mehr an die Lehrenden als an die Lernenden zu richten scheinen und diese für die Studierenden nicht leicht zu verstehen und zu kontextualisieren sind.

Dass es sich aber lohnt, der Selbsteinschätzung und metakognitiven Reflexionen im Unterricht Raum zu geben, wird von allen bestätigt:

\begin{abstract}
Aber es ist auch wahr, dass man als Lehrerin, genau erklären muss, welche Kompetenzen erreicht werden sollen, was man können muss, sodass der Lerner in diesem Prozess Verantwortung übernimmt und nicht nur die Lehrerin agiert und der Schüler passiv aufnimmt. Auf diese Weise wird er aktiv und kann auch die Lehrerin kontrollieren, ob tatsächlich in die richtige Richtung gearbeitet wird; aber auch die Lehrerin hat einen besseren Überblick, was zu tun ist, da man manchmal selbst das Ziel aus den Augen verliert, mit etwas anfängt, um dann ganz wo anders anzukommen. Es erlaubt also beiden mehr Transparenz, mehr Klarheit.*
\end{abstract}

\title{
$6 \quad$ Fazit
}

An der Basis all dieser perplessita ${ }^{x v}$ finden wir die Schwierigkeit der Lehrenden, sich von der Tradition wegzubewegen, die „Fremdsprache“ als Lerngegenstand zu betrachten und das Sprachenlernen im Sinne einer funktionalen Mehrsprachigkeit zu sehen und zu einem handlungsorientierten Ansatz überzugehen. Es besteht ein großer Bedarf an gezielten, sprachenübergreifenden Lehrerfortbildungen, aber auch an Peergruppen, in denen sich die Lehrpersonen in regelmäßigen Abständen austauschen können. Das ESP muss, wie bereits gesagt, vom gesamten Kollegium getragen werden, d.h. möglichst alle Dozentinnen, die mit Sprachunterricht zu tun haben, sollten gemeinsam planen, so lässt sich sicherlich auch der notwendige Zeitaufwand auf die verschiedenen Fächer verteilen. Mehr noch, die Einführung des ESP sollte als schulinternes Projekt präsentiert, von der Direktion getragen und mit den notwendigen finanziellen Mitteln unterstützt werden. Darüber hinaus 
sollten die involvierten LehrerInnen kontinuierlich von Fachleuten oder, wie das Projekt aus Turin vorsieht, von dafür ausgebildeten Tutoren (Ferrero 2003) begleitet werden. Es ist nicht sinnvoll, sich auf die Eigeninitiative und das Engagement einzelner Lehrpersonen zu stützen. In der Ausarbeitung eines längerfristigen Projektes können dann sicherlich effiziente Lösungen für die verschiedenen Problempunkte, nicht zuletzt für den anfänglichen Mehraufwand an Vorbereitungsstunden, gefunden werden. Das gesamte System (Direktion, LehrerInnen und auch Eltern) sollte sich, wie Thürmann betont (2007: 33), im Vorfeld einer Einführung des ESP fragen: „Was müssen wir an unserer Schule und in unserem Unterricht ändern, damit Portfolio-Arbeit ihr volles Potenzial entwickeln kann?" und ich würde noch hinzufügen „Sind wir wirklich dazu bereit?“, da jede Art von Neuerung mit einer mitunter verunsichernden Übergansphase einhergeht.

Weiters erscheint es zielführend, das Prinzip der erweiterten Leistungsbeurteilung soweit ernst zu nehmen, dass das ESP in der Notengebung berücksichtigt wird, was die Motivation von Seiten der Lernenden stärken, aber auch das Instrument ESP im Schulsystem besser integrieren würde. Schließlich sollten Lehrbücher diesen neuen Bedürfnissen und dem Konzept der Portfolio-Arbeit besser angepasst werden.

Zwischen Theorie (Sprachenpolitik, Schulbehörden) und Praxis (Arbeitsalltag der Lehrenden) gibt es eine große Kluft: Wenn z.B. im italienischen Bericht über die Durchführung des Aktionsplans (European Commission 2006: 6) zu lesen ist, dass in Italien „more and more" mit dem ESP gearbeitet wird, dass 10 Modelle akkreditiert und Fortbildungen organisiert wurden, dann erscheint diese Berichterstattung etwas dürftig und oberflächlich. Die von mir interviewten Lehrerinnen haben praktische alle nach unserem Projekt aufgehört, mit dem ESP zu arbeiten. ${ }^{\text {vvi }}$ Das ESP ist also noch nicht Teil des Schulalltags!

Für eine erfolgreiche Arbeit mit dem ESP ist ein, wie schon vom Konstruktivismus geforderter Paradigmenwechsel in unserer Lehr- und Lerntradition (Bleyhl 2000) notwendig und dieser erfordert Zeit. Dass sich diese Zeitinvestition lohnt, haben die positiven Reaktionen von Seiten der an den Projekten beteiligten StudentInnen und SchülerInnen gezeigt, aber der Weg ist eben noch lang.

\section{Literatur}

Bilotto, Andrea (2000): Pilot experimentation of the European Language Portfolio at the University of Calabria. In: Csillaghy, Andrea \& Gotti, Maurizio (Hrsg.): Le Lingue nell'Università del Duemilla. Udine: Forum, 321-331.

Brüll, Claudia (1999): Die Sprachenpolitik der Europäischen Union. Europa Aktuell [http://www.europa-digital.de/text/aktuell/dossier/sprachen/sprache1.shtml; 20.09.2008].

Civegna, Klaus; Duregger, Josef; Gelmi, Rita; Mussner, Rosmarie \& Siverio, Carmen (Hrsg.) (2007): Sprachen leben und erleben - Vivere le lingue - Viver la rujenedes. Bozen: PI. 
Coucil for Cultural Cooperation (2000): European Language Portfolio (ELP). Principles and guidelines. [http://www.coe.int; 20.09.2008].

Council of Europe (1998): Recommendation No. R (98) 3 of the Committee of Ministers to Member States on Access to Higher Education. [http:/ / wallis.kezenfogva.iif.hu/eu konyvtar/projektek/vocational_rehabilitiation; 20.09.2008].

Council of Europe (2000): Resolution on the European Language Portfolio. Conference of the Ministers of Education. [http://www.coe.int.; 20.09.2008].

Europaparlament (2000), Charta der Grundrechte. [http://www.europarl.europa.eu; 20.09.2008].

Europarat für kulturelle Zusammenarbeit (2001): Gemeinsamer europäischer Referenฐrahmen für Sprachen: lernen, lehren, beurteilen. Berlin [u.a.]: Langenscheidt.

European Commission (2006): Follow-up of the Action Plan on language learning and linguistic diversity. National Report Template - Italy. [http://www.coe.int.; 20.09.2008].

ESP - Modelle zum Herunterladen. [http://www.coe.int.; 20.09.2008].

Ferrero Camera, Rosalia (2003): Il Monitoraggio del modello piemontese. In: Ufficio Scolastico Regionale per il Piemonte del Miur (Hrsg.): Portfolio Europeo delle Lingue Un'esperienza piemontese. Grugliasco: Grafiche San Rocco, 51-82.

Ghislandi, Patrizia (2004): Focus Group - Modello didattico-organizzativo per i corsi online dell'Università di Trento. Unveröffentlichter Abschlussbericht Trento.

Glaboniat, Manuela; Müller, Martin; Rusch, Paul; Schmitz, Helen \& Wertenschlag, Lukas (2002): Profile deutsch. Gemeinsamer europäischer Referenzrabmen. Lernzielbestimmung, Kannbeschreibungen, Kommunikative Mittel. Berlin [u.a.]: Langenscheidt.

Goethe-Institut (2002): PerV oi. Zeitschrift für Deutschlehrerinnen und Lebrer in Italien. Rom: Internationes, Januar bis Juni 2002.

Keller, Susy (2002), Die Portfolio-Erprobung in der Schweiz. In: PerV oi. Zeitschrift für Deutschlehrerinnen und Lehrer in Italien. Rom: Internationes: Januar bis Juni 2002, 19-20.

Kommission der Europäischen Gemeinschaften (2003): Förderung des Sprachenlernens und der Sprachenvielfalt: Aktionsplan 2004 - 2006. Brüssel. [http://www.coe.int.; 20.09.2008].

Kommission der Europäischen Gemeinschaften (2007): Bericht über die Durchführung des Aktionsplans „Förderung des Sprachenlernens und der Sprachenvielfalt“. [http://www.coe.int.; 20.09.2008].

Little, David \& Perclovà, Radka (2000): The European Language Portfolio: a guide for teachers and teacher trainers. [http://www.coe.int.; 20.09.2008].

Little, David \& Simpson, Barbara, ELP (2003): The intercultural component and Learning how to learn. [http://www.coe.int.; 20.09.2008].

Little, David (2002), The European Language Portfolio in use: nine examples. [http://www.coe.int.; 20.09.2008].

Mariani, Luciano (2002): Otto Sfide del Portfolio. Alcune implicazioni pedagogiche di uno strumento potenzialmente innovativo. In: PerVoi. Zeitschrift für Deutschlehrerinnen und Lehrer in Italien. Rom: Internationes: Januar bis Juni 2002, 12-14.

Mariani, Luciano (2003): Il portfolio come strumento di valutazione formativa. [http://www.crtvolta.it/materiali/materiali.asp; 20.09.2008]. 
Ministero dell'Isttruzione, dell'Università e della Ricerca (2005) : Il Portfolio Europeo dele Lingue: informazioni di base. [http://www.pubblica.istruzione.it/argomenti/ portfolio/documenti.shtml; 20.09.2008].

Palamidesi, Flora (2002): Il Portfolio linguistico nella regione Umbria (1998 - 2000) nel quadro delle attività del Consiglio d'Europa. In : PerV oi. Zeitschrift für Deutschlehrerinnen und Deutschlebrer in Italien. Rom: Internationes, Januar bis Juni 2002, 5-7.

Parlamento europeo e del Consiglio (2004): Decisione N. 2241/2004/CE Del Parlamento Europeo e del Consiglio relativa ad un quadro comunitario unico per la trasparenza delle qualifiche e delle competenze. Gazzetta ufficiale dell’Unione europea [http://europa.eu.int; 20.09.2008].

Schärer, Rolf (2004): A European Language Portfolio. From piloting to implementation (2001-2004). Consolidated report - final version. [http://www.coe.int; 20.09.2008].

Schärer, Rolf (2000): Final report. A European Language Portfolio. Pilotproject phase 1998-2000. [http://www.coe.int; 20.09.2008].

Schneider, Günther (1999): Wozu ein Sprachenportfolio? Funktionen und Merkmale des Europäischen Sprachenportfolios (Schweizer Version). [www.unifr.ch/ids/Portfolio/pdfs/aufsatz-gu-wozu-pf.pdf; 20.09.2008].

Schneider, Günther \& Lenz, Peter (2001): European Language Portfolio: Guide for Developers. [http://www.coe.int; 20.09.2008].

Stricker, Sabine (2006): Selbstbestimmung, Selbsteinschätzung und Selbsterfahrung Autonomie im DaF-Unterricht oder Was passiert, wenn sich Sprachlernen verselbständigt? In: Abel, Andrea; Stulfesser, Mathias \& Putz, Magdalena (Hrsg.)(2006): Plurilinguismo in Europa/Mehrsprachigkeit in Europa/Multilingualism across Europe, Bolzano: EuroResearch, 183-191.

Stricker, Sabine (2007): SprachenportfolioUnitn. Sprachkompetenzen sichtbar machen. Trento: Editrice Uni Service.

Stricker, Sabine (2008): Portfolio, Portfolio delle mie brame, chi sono gli studenti del nostro reame? Il valore documentativo e pedagogico del Portfolio Europeo delle Lingue. In: Atti del convegno: L'AICLU e la politica linguistica nelle università italiane. Università di Parma im Druck (2008).

Ufficio Scolastico Regionale per il Piemonte del Miur (2003) : Portfolio Europeo delle Lingue Un'esperienza piemontese. Grugliasco: Grafiche San Rocco.

i Aussage einer Lehrerin der Fokusgruppe.

ii Viele ESP-Modelle können unter http://www.coe.int kostenlos herunter geladen werden.

iii Portfolio Europeo delle Lingue 26.2002, S. 85

iv 65.2004 Model for learners in primary education und 69.2005 Model for learners in lower secondary education, diese zwei ESP aus Südtirol widmen der Kompetenz der Mediation zwischen zwei Sprachen Platz.

v 48.2003 IT Model for learner in vocational sectors; 29.2002 IT Model for learners in higher education.

vi $\quad 54.2003$ Model for learners aged $15+$ and adults. 
vii Ich frage mich auch, inwieweit das ESP in der Arbeitswelt überhaupt bekannt ist und ob eine Informationskampagne zum ESP in der Arbeitswelt vorgesehen ist?

viii Die mit* gekennzeichneten Zitate sind Transkriptionen (einfache literarische Transkription mit den gängigen Satzzeichen) und Übersetzungen der Fokusgruppe mit Lehrerinnen aus verschiedenen Schulen von Trient. Da auch eine Französischlehrerin teilnahm, war die Kommunikationssprache Italienisch.

ix Hier spreche ich nur von Lehrerinnen, da nur Frauen anwesend waren. Die Teilnehmerinnen waren neben dieser Fokusgruppe auch in einer Arbeitgruppe zum Thema „Il portfolio europeo delle lingue come ponte tra la scuola superiore e l'università " tätig, d.h. das Thema ESP war für sie nicht völlig neu.

x Mehr zu diesem Aspekt in: Stricker (2006).

xi Die Schulreform nennt sich "Moratti” nach der damaligen Ministerin Letizia Moratti. Das Kompetenzportfolio ist mittlerweile schon wieder abgeschafft worden.

xii Herausforderungen.

xiii Mehr dazu in: Stricker (2008).

xiv Mehr dazu in: Stricker (2006).

xv Der Begriff perplessità könnte einfach mit Zweifel übersetzt werden, doch trägt er noch feinere Bedeutungen in sich, die auch Nichtverstehen, Stirnrunzeln und stutzig werden meinen.

xvi Die Zahl der befragten Lehrerinnen ist, wie bereits gesagt, statistisch nicht relevant, hat aber als Stichprobe seine Bedeutung und wird auch durch die Tatsache bestätigt, dass ich in meiner Lehrtätigkeit an der Universität Trient noch keine StudentIn mit einem im Gymnasium erstellten ESP getroffen habe. 


\title{
Die traditionelle Lehrwerkanalyse und die Diskursanalyse -
}

\author{
Zwei Methoden zur inhaltlichen Analyse von Lehrwerken für \\ Integrationskurse
}

Anja Ucharim (Leiprig)

\begin{abstract}
Der vorliegende Beitrag basiert auf einem Dissertationsprojekt in den Kulturstudien des Faches Deutsch als Fremdsprache, in dem der Zusammenhang von Wirklichkeitskonstruktion, Integrationskonzept und pädagogisch-landeskundedidaktischem Ansatz in Lehrwerken für Integrationskurse herausgearbeitet werden soll. Im Zentrum steht dabei die Analyse der in den Lehrwerken hergestellten Wirklichkeit, genauer gesagt, der sprachlichen und visuellen Mittel, mit deren Hilfe diese Wirklichkeit konstituiert wird. Zur Untersuchung dieser Mittel wird eine Methode aus der Schulbuchforschung adaptiert: die thematische Diskursanalyse, die von den Frankfurter Erziehungswissenschaftlern Höhne, Kunz und Radtke für die Analyse von Schulbüchern entwickelt (1999 und 2005) und bislang ebenso wenig wie andere diskursanalytische Verfahren zur Analyse landeskundlicher Inhalte von Lehrwerken für Deutsch als Fremd- und Zweitsprache angewendet worden ist. Um den Zusammenhang von Wirklichkeitskonstruktion, Integrationskonzept und pädagogisch-landeskundedidaktischem Ansatz zu erschließen, orientiert sich das Projekt hingegen an Leitfragen, die als traditionelle Vorgehensweise der Lehrwerkanalyse gelten können. Der Beitrag gliedert sich in zwei Teile: Im ersten Teil wird das Projekt skizziert, im zweiten Teil die Forschungsmethodik vorgestellt und anhand einer Beispielanalyse illustriert.
\end{abstract}

\section{Das Dissertationsprojekt}

Bei der Vorstellung des Dissertationsprojekts soll zunächst auf seine theoretische Verortung in den Kulturstudien des Faches Deutsch als Fremdsprache und seine Zielsetzung eingegangen werden. Anschließend werden die erkenntnisleitenden Hypothesen dargelegt und Vermutungen hinsichtlich der Ergebnisse angestellt.

Thema des Dissertationsprojekts ist die Repräsentation von Wirklichkeit in Lehrwerken für Integrationskursei; herausgearbeitet werden soll der Zusammenhang von Wirklichkeitskonstruktion, Integrationskonzept und pädagogisch-landeskundedidaktischem Ansatz. Den Ausgangspunkt für die Erschließung dieses Zusammenhangs bildet die Analyse der in den Lehrwerken hergestellten Wirklichkeit im Hinblick auf Heterogenität. 
Globalisierungsprozesse und weltweite Migrationsbewegungen bedingen eine forcierte Auflösung kulturell homogener Gesellschaften. ii Die Pluralisierung von Gesellschaften geht einerseits mit der Erodierung bislang - vorwiegend - gültiger Deutungsmuster einher und ermöglicht andererseits die Etablierung einer Vielzahl heterogener Selbst- und Wirklichkeitsdeutungen.iii Dieser Entwicklung trägt die sich in jüngster Zeit konstituierende Kulturwissenschaft des Faches Deutsch als Fremdsprache Rechnung (vgl. im Folgenden Altmayer 2006: 51 ff.): Sie geht in Anlehnung an den sozialen Konstruktivismus davon aus, dass Wirklichkeit nicht unmittelbar, sondern nur als gedeutete zugänglich ist und damit ein Konstrukt darstellt. ,Sozial' bedeutet in diesem Zusammenhang, dass ein Individuum diesen Deutungs- und Konstruktionsprozess nicht autonom vollzieht, sondern unter Rückgriff auf gesellschaftlich verankertes, in der Sozialisation erworbenes und in Diskursen $^{\text {iv }}$ als bekannt vorausgesetztes Wissen: auf kulturelle Deutungsmuster, deren Gesamtheit die Kultur einer - nicht als national zu definierenden - Kommunikationsgemeinschaft bildet. Kultur kann demnach als kollektiver Wissensfundus verstanden werden, und es ist evident, dass ein solcher Fundus mehrere und auch einander widersprechende Muster für unterschiedliche Wirklichkeitsdeutungen enthält. Wirklichkeit löst sich damit ,,aus kulturwissenschaftlicher Sicht in unzählige subjektive Perspektiven und diskursive Sinnzuschreibungen" (Altmayer 2006: 53) auf. Angesichts der sozial-konstruktivistischen Prämisse und des grundsätzlich auf Heterogenität hin angelegten Kulturbegriffs der kulturwissenschaftlich fundierten Landeskunde des Faches Deutsch als Fremdsprache kann die Annahme einer homogenen Wirklichkeit nur mehr als obsolet bezeichnet werden; Wirklichkeit, verstanden als Produkt subjektiver Deutung(en), erscheint stattdessen als komplex, ambivalent und vielschichtig, kurz: als heterogen. Das bedeutet für die Konzeption von, deutscher Wirklichkeit‘ als Gegenstand landeskundlichen Lernens in Lehrwerken für Deutsch als Fremd- und Zweitsprache, dass auch für diese Heterogenität einzufordern ist.

Daher soll zunächst untersucht werden, ob diese Lehrwerke ein vielschichtiges oder ein lediglich eindimensionales Bild von Wirklichkeit entwerfen, welche Rolle Subjekten in dieser Wirklichkeit zugewiesen wird und mit Hilfe welcher sprachlicher und visueller Mittel dies geschieht. Anschließend soll eine Verbindung zwischen der jeweiligen Wirklichkeitskonstruktion, dem Integrationskonzept und dem pädagogischen sowie dem landeskundedidaktischen Ansatz eines Lehrwerks nachgewiesen werden. Als Bezugswissenschaften sind hier neben den Kulturwissenschaften die Migrationssoziologie, die Didaktik der Landeskunde und die interkulturelle Pädagogik heranzuziehen. Da Lehrwerke, indem sie gesellschaftliche Diskurse reflektieren, zur Reproduktion von Machtstrukturen beitragen können (vgl. Höhne, Kunz \& Radtke 1999: 25), sollen in einem weiteren Schritt die in der Analyse ermittelten Wirklichkeitskonstruktionen, Rollenzuweisungen, Integrationskonzepte sowie die pädagogischen und landeskundedidaktischen Ansätze jeweils mit korrespondierenden Entwürfen in politischen und didaktischen bzw. politisch initiierten didaktischen Texten ${ }^{v}$ verglichen werden, um einen Zusammenhang zwi- 
schen den Diskursen der Lehrwerke und denen des öffentlichen Bereichs herauszuarbeiten.

Hinsichtlich der Ergebnisse wird angenommen, dass die diskursive Konstruktion von Wirklichkeit als homogen oder heterogen Konsequenzen für die Rolle hat, die den Lernenden in dieser Wirklichkeit jeweils zukommt, und mit unterschiedlichen Konzepten von Integration, Pädagogik und Landeskundedidaktik korreliert. Im Einzelnen lassen sich folgende Hypothesen aufstellen:

1. Die diskursive Konstruktion einer homogenen Wirklichkeit trägt den Lernenden spezifische Muster der Selbst- und Wirklichkeitsdeutung an, d. h. den Lernenden wird potentiell suggeriert, dass es ausschließlich die im Lehrwerk präsentierte Deutungsmöglichkeit für , die deutsche Wirklichkeit' und die eigene Rolle in dieser Wirklichkeit gebe. Eine als homogen konstruierte Wirklichkeit verweist auf ein Konzept von Integration als Assimilation, auf die Kontinuität der ,Ausländerpädagogik', die den Zugewanderten herkunftsbedingte ,kulturelle“ Defizite unterstellt (vgl. Krüger-Potratz 2005: 121 ff.), und auf eine interkulturelle Landeskundedidaktik, die einen essentialistischen Kulturbegriff zugrunde legt. Lernende werden hier vorwiegend als Repräsentanten ihrer - als homogen verstandenen - Kultur betrachtet (vgl. Altmayer 2006: 49).

2. Demgegenüber ermöglicht die diskursive Konstruktion einer heterogenen Wirklichkeit den Lernenden eigenständige Selbst- und Wirklichkeitsdeutungen. Sie verweist auf ein Konzept von Integration als Partizipation, also als „,g]leichberechtigte Teilhabe an den gesellschaftlichen Entscheidungsprozessen und Ressourcen“ (Krüger-Potratz 2005: 262), auf Ansätze einer auf dem Prinzip der Gleichheit in der Differenz beruhenden interkulturellen Bildung (vgl. Krüger-Potratz 2005: 148 ff.) und auf den kulturwissenschaftlich fundierten Ansatz des kulturellen Lernens, der die Individualität der Lernenden betont und deren Befähigung zur Partizipation an deutschsprachigen Diskursen anstrebt (vgl. Altmayer 2006: 56).

Ausgehend von den beiden Hypothesen soll die Interdependenz von Wirklichkeitskonstruktion, Integrationskonzept und pädagogisch-landeskundedidaktischem Ansatz in Lehrwerken für Integrationskurse herausgearbeitet werden. Erste Analyse-Ergebnisse weisen darauf hin, dass sich die vermuteten Zusammenhänge bestätigen werden; wichtige Aufschlüsse sind vor allem hinsichtlich der diskursiven Konstruktion von Wirklichkeit im Medium Lehrwerk zu erwarten.

\section{Die Methodik}

In den folgenden Abschnitten werden unterschiedliche Methoden zur Analyse von Lehrwerken vorgestellt; die beiden Erkenntnisinteressen des Projekts - die Konstruktion von Wirklichkeit einerseits und der Zusammenhang von Wirklich- 
keitskonstruktion, Integrationskonzept und pädagogisch-landeskundedidaktischem Ansatz andererseits - erfordern dabei die Kombination zweier Verfahren.

\subsection{Traditionelle Methoden der Lehrwerkanalyse}

Aufgrund seiner Fokussierung landeskundlicher Aspekte bezieht sich das Projekt methodisch auf die inhaltliche Lehrwerkforschung, die sich vor allem hermeneutischer Verfahren bedient. Empirisch-quantitative Methoden, die lediglich die Häufigkeit der Thematisierung ausgewählter Inhalte in Lehrwerken messen können (vgl. Bungvi 1977: 114-145), werden in der landeskundlichen Lehrwerkforschung gegenwärtig kaum angewendet. ${ }^{\text {vii }}$

Im Gegensatz zu empirischen ermöglichen hermeneutische Verfahren die Erfassung impliziter Aussagen und Wertungen sowie gesellschaftlicher Hintergründe und Machtstrukturenviii (vgl. Maijala 2004: 49). Damit gewährleisten sie einerseits eine ganzheitliche und differenzierte Darstellung der Inhalte (vgl. Krumm 1994: 25) und machen andererseits die einem Lehrwerk zugrunde liegenden Prämissen transparent (vgl. Neuner 1994: 112). Allerdings sind die Ergebnisse nicht nur an die Analysefähigkeit, sondern auch an die theoretische oder didaktische Position der Forscherin oder des Forschers gebunden (vgl. Krumm \& Ohms-Duszenko 2001: 1034). Um eine intersubjektive Nachvollziehbarkeit zu gewährleisten, ist die Offenlegung der theoretischen Verortung und die fundierte Herleitung der Analysekriterien daher ebenso unumgänglich (vgl. Abendroth-Timmer 1998: 28; Funk 1994: 109) wie eine dezidierte Rückbindung der Ergebnisse an das Text- oder Bildmaterial.

Die Analyse landeskundlicher, aber auch linguistischer oder didaktischer Gegenstände kann sich entweder an zuvor erstellten Kriterienkatalogen ${ }^{\text {ix }}$ oder an erkenntnisleitenden Fragestellungen ${ }^{x}$ orientieren. Während der Vorteil der Kataloge darin besteht, ein Vergleichsraster zu liefern (vgl. Abendroth-Timmer 1998: 27; Funk 1994: 109), können mit Hilfe von Leitfragen Inhalte differenzierter und damit exakter erfasst werden. Da diese Fragen immer vor dem Hintergrund spezifischer Theorien oder der Unterrichtspraxis entwickelt werden, sind theoretische Positionierung und normative Ansprüche - häufig im Gegensatz zu den Katalogen (vgl. Abendroth-Timmer 1998: 28; Funk 1994: 109; Thimme 1996: 95) - in der Regel transparent. Nachteile liegen jedoch auch hier in der Gefahr der Subjektivität (vgl. Maijala 2004: 54) und in der schwierigeren Handhabbarkeit für die Praxis, denn ein schematischer Vergleich ist hier nicht möglich. Der erste Nachteil kann allerdings durch die oben beschriebenen Maßnahmen relativiert und der zweite durch den Vorteil der Leitfragen, komplexe Inhalte und Zusammenhänge aufzeigen zu können, mehr als ausgeglichen werden.

Das eigene Projekt wird das traditionelle Verfahren der Lehrwerkanalyse mit Hilfe von Leitfragen anwenden, um den Zusammenhang von Wirklichkeitskonstruktion, Integrationskonzept und pädagogisch-landeskundedidaktischem Ansatz zu erschließen. Dabei werden die Leitfragen, die das Vorverständnis der Verfasse- 
rin offenlegen sollen, anhand von Theorien aus der - kulturwissenschaftlichen Landeskunde und ihrer Didaktik, der Migrationssoziologie und der interkulturellen Pädagogik entwickelt. Die Konstruktion von Wirklichkeit selbst hingegen soll mit Hilfe des im folgenden Abschnitt vorgestellten diskursanalytischen Verfahrens nachvollzogen werden.

\subsection{Die thematische Diskursanalyse}

Die Diskursanalyse, eine - mittlerweile! - gleichfalls als hermeneutisch zu bezeichnende Methode (vgl. Höhne et al. 1999: 62; Keller 2004: 72), wird in jüngster Zeit im Rahmen der Schulbuchforschung erprobt und ist bislang noch nicht zur Analyse landeskundlicher Inhalte von Lehrwerken für Deutsch als Fremd- und Zweitsprache angewendet worden. Den Einsatz dieses Verfahrens legen aber folgende Gründe nahe:

1. Das Projekt ist theoretisch in der kulturwissenschaftlich fundierten Landeskunde des Faches Deutsch als Fremdsprache zu verorten, die sich unter anderem auch der Diskursanalyse zur Ermittlung kultureller Deutungsmuster in medialen Texten und Bildern bedient. Die Anwendung dieser Methode auf LehrwerkTexte und -Bilder ergibt sich daher bereits aus der wissenschaftlichen Positionierung des Projekts.

2. Im Bereich der Schulbuchforschung wurde die Methode zur Untersuchung der Darstellung von Migrantinnen und Migranten in Lehrbüchern für Sach- und Sozialkunde eingesetzt und hat dort aufschlussreiche Ergebnisse erbracht (vgl. Höhne, Kunz \& Radtke 1999 und 2005). Die Adaption dieser bereits bewährten Methode für den Bereich des Deutschen als Fremd- und Zweitsprache bietet sich deswegen - und selbstverständlich auch aufgrund des gemeinsamen Gegenstands Lehrwerk - in besonderem Maße an.

3. Das Erkenntnisinteresse (kritischer) diskursanalytischer Verfahren richtet sich auf die diskursive Konstruktion und Reproduktion gesellschaftlicher Machtbeziehungen. Damit ergeben sich nicht nur Parallelen zu der in der Lehrwerkforschung schon immer erhobenen Forderung nach der Berücksichtigung gesellschaftlich-institutioneller Faktoren (vgl. Neuner 1979: 12 ff. und 1994: 12 ff.), sondern auch zum Erkenntnisinteresse des Projekts, in dem politische und politisch initiierte didaktische Texte in Beziehung zu den Ergebnissen der Lehrwerkanalyse gesetzt werden, um die Einbindung der Lehrwerke in ihr soziopolitisches Bedingungsgefüge offenzulegen.

Von der Vielzahl diskursanalytischer Methoden orientiert sich das Projekt an der thematischen Diskursanalyse (tDA ${ }^{\mathrm{x}}$ ), die von den Frankfurter Erziehungswissenschaftlern Thomas Höhne, Thomas Kunz und Frank-Olaf Radtke entwickelt und erstmals zur Analyse von Schulbüchern eingesetzt worden ist. Ihr Erkenntnisinteresse besteht - im Gegensatz etwa zu Bung oder Ammer - nicht in der Beurteilung 
von Lehrwerken hinsichtlich der, Wahrheit', Ausgewogenheit oder ,Objektivität ihrer Inhalte (vgl. Höhne et al. 1999: 38), sondern richtet sich vielmehr auf das Zustandekommen und die Struktur von Lehrbuchdiskursen (vgl. auch im Folgenden, Höhne, Kunz \& Radtke 2005: 27 ff.). Letztere basiert auf der regelhaften Anordnung unterschiedlicher Konstituenten eines thematischen Diskurses: typischen semantischen Merkmalen, intra- und interdiskursiven Verknüpfungen und Verweisen auf gesellschaftliches und dominantes Wissen. Dieses Wissen findet, nachdem es politisch-administrativ legitimiert und als wissenswert beurteilt worden ist, in die Lehrbücher Eingang, wird aber gleichzeitig auch von ihnen hervorgebracht und distribuiert. Häufig liegt es lediglich implizit vor ${ }^{\mathrm{xii}}$ und bedarf daher der Rekonstruktion mit Hilfe eines spezifischen Instrumentariums.

Dem analytischen Instrumentarium von Höhne, Kunz und Radtke liegen unterschiedliche linguistische Teildisziplinen, Ansätze und Theorien zugrunde: Textlinguistik, Semantik, Strukturalismus und Argumentationstheorie, aber auch Textund Bild-Semiotik (vgl. Höhne et al. 2005: 34f.). Die Vielfalt der theoretischmethodischen Bezugspunkte ermöglicht sowohl die Erfassung der unterschiedlichen Funktionsebenen eines Diskurses (vgl. Höhne et al. 2005: 34) als auch die Untersuchung sprachlicher und bildlicher Zeichen mit einem vergleichbaren Instrumentarium (vgl. Höhne et al. 1999: 68). Damit trägt das Verfahren dem Charakter des Mediums Lehrbuch Rechnung, das eine „,komplexe Montage aus Text-, Bild- und Graphikelementen darstellt" (Höhne et al. 1999: 68). Entsprechend berücksichtigt das Ablaufschema der $\mathrm{tDA}$ xiii vier Dimensionen: 1. die Diskursform, 2. intradiskursive semantische Verknüpfungen, 3. interdiskursive Anschlüsse und 4. die Bildebene (vgl. Höhne et al. 2005: 33f.). xiv

Die Analyse der Diskursform, der medialen Form eines Diskurses, orientiert sich an der Critical Layout Analysis von Kress und Van Leeuwen, denen zufolge Bedeutungen von Texten und Bildern nicht nur sprachlich oder visuell, sondern auch durch ihr Arrangement auf einer Seite hergestellt werden (vgl. Kress \& Van Leeuwen 1995: 25 und 42). Um die Aussage eines Textes oder Bildes präzise erfassen zu können, sind bei der Analyse von Printmedien demnach auch die Mittel des Layouts zu berücksichtigen: Plazierung, Markierung und Rahmung (vgl. auch im Folgenden Kress et al. 1995: 25ff.). So kann die Rechts-Links-Plazierung von Textoder Bild-Elementen Informationen hinsichtlich ihrer Aktualität strukturieren, die Zentrum-Peripherie-Plazierung hinsichtlich des Informationswerts und die ObenUnten-Plazierung hinsichtlich der Detailliertheit. Markierung erfolgt mit Hilfe unterschiedlicher visueller Mittel, die die Aufmerksamkeit der Rezipierenden unterschiedlich stark auf einzelne Elemente lenken sollen, z. B. Positionierung im Vorder- oder Hintergrund, Farbkontraste, Farbton, Bildschärfe, aber auch - wie Höhne, Kunz und Radtke ergänzend anmerken (vgl. 2005: 36) - Typographie oder Schriftgröße. An der Rahmung schließlich lässt sich ablesen, ob und in welchem Grad einzelne Text- und Bild-Elemente miteinander verbunden sind; dabei können trennende und verbindende Rahmungsmittel unterschieden werden. Der bedeutungskonstituierende Effekt des Layouts begründet seine separate Untersuchung: 
Die formale Erscheinungsweise eines Lehrwerk-Diskurses nämlich ist das Ergebnis eines komplexen Produktionsprozesses, in dem nach spezifischen Kriterien, didaktischen Prinzipien und Zielsetzungen Entscheidungen für eine bestimmte Präsentationsform und gegen eine andere getroffen wurden (vgl. Höhne et al. 1999: 67f.).

An die Analyse der Diskursform schließt sich die Diskursanalyse der Textdokumente an, die sowohl die intradiskursiven als auch die interdiskursiven Relationen der sprachlichen Zeichen berücksichtigt. ${ }^{\mathrm{xv}}$ Die intradiskursiven Relationen der sprachlichen Zeichen sollen mit Hilfe unterschiedlicher Operationen ermittelt werden. An erster Stelle steht der Prozess der Delinearisierung der einzelnen Dokumente, der der Analyse von Monosemierungsprozessen dient (vgl., auch im Folgenden, Höhne et al. 2005: 36). Monosemierung meint die Herstellung eines eindeutigen Sinns, die im Lehrbuch durch die Text-Bild-Komposition bewirkt wird. Durch die Delinearisierung, d. h. durch die Auflösung des Text-Bild-Zusammenhangs, werden diese Vereindeutigungsprozesse rückgängig gemacht, so dass jedes Text- bzw. Bild-Dokument für sich genommen und im Hinblick auf mögliche weitere thematische Anschlüsse betrachtet werden kann. Das gleiche Ziel verfolgen strukturalistische Operationen. Eine besondere Aufmerksamkeit wird den Eingangssequenzen zuteil, worunter nicht nur die Einleitungssätze eines Lehrbuchtextes, sondern unter anderem auch Überschriften und Aufgabenstellungen gefasst werden (vgl. Höhne et al. 1999: 35 f.). Da Eingangssequenzen eine leitthematische Funktion besitzen, sind sie für die thematische Bestimmung eines Diskurses von Bedeutung (vgl. Höhne 2004: 406), und da sie den Rezipierenden eine bestimmte Lesart eines Textes oder Bildes nahelegen, tragen sie wesentlich zur Monosemierung bei. Weiterhin ist das Konzept der Kobäsion relevant (vgl. Höhne et al. 2005: 37), denn durch die Analyse der unterschiedlichen Kohäsionsmittel wird die „Rekonstruktion der für einen Diskurs konstitutiven semantischen Elemente“ sowie ihrer Verknüpfungen, teilweise aber auch die „Rekonstruktion impliziten Diskurswissens“ ermöglicht ${ }^{\mathrm{xvi}}$ (vgl. Höhne et al. 2005: 33).

Innerhalb der Analyse der intradiskursiven Relationen kann besonders die Differenzmaschine als elaboriertes Verfahren im engeren Sinne bezeichnet werden (vgl. im Folgenden Höhne et al. 2005: 38 ff.). Mit ihrer Hilfe lassen sich die Strukturen von Prädikationen und Differenæsetzungen ermitteln, zwei grundlegenden und komplementären Prozessen, die die thematische Entwicklung eines Diskurses befördern, indem sie durch Auswahl und Verknüpfung semantischer Elemente spezifische Bedeutungen erzeugen und festschreiben. Die durch Prädikationen hergestellten Verknüpfungen zwischen Lexemen können dabei nicht nur in expliziten oder impliziten Merkmalszuweisungen, sondern auch in konträren bzw. kontradiktorischen Beziehungen, Differenzsetzungen, bestehen. Explizite und implizite Prädikationen können mit dem Konzept der Isotopie erfasst werden, das wiederum auf dem Konzept der semantischen Merkmale beruht. Für eine Isotopie sind vor allem semantische Äquivalenzen konstitutiv; allerdings können auch, wie anhand des folgenden Beispielsatzes deutlich wird, semantische Differenzen eine Rolle spielen: 
So besitzen in dem Satz ,Erkan traf seine deutschen Freunde. Erkan und seine Freunde gleichermaßen die Eigenschaften [ + menschlich] und [+ lebendig], während, indem nur den Freunden die Eigenschaft [+ deutsch] zukommt, Unterschiede markiert werden. Detailliert darstellen lassen sich diese Prädikations- und Differenzstrukturen durch das Modell der Differenzmaschine, das Höhne, Kunz und Radtke in Anlehnung an das semiotische Viereck von Greimas entwickelt haben und das im Folgenden lediglich verkürzt dargestellt werden kann: Auf einer Achse werden zwei Lexeme (z. B. ,deutsch` - ,türkisch), die ein gemeinsames Merkmal besitzen (z. B. [+ Nationalität]), konträr zueinander in Beziehung gesetzt. An diese beiden konträren Lexeme schließt sich jeweils eine vertikale Achse an, auf der weitere Oppositionen, die sich in einem Dokument finden, eingetragen werden können (z. B. ,wir ${ }^{6}$, ihr'). Diese dienen der näheren Bestimmung der Ausgangsopposition und formieren eine Struktur der Differenzsetzungen. Prädikationen und Differenzen können durch unterschiedliche Modalitäten - Faktizität, Möglichkeit oder Restriktion - zusätzlich entweder flexibilisiert oder affirmiert werden.

Die Analyse der intradiskursiven Relationen der sprachlichen Zeichen dient vor allem der Rekonstruktion diskurskonstitutiver semantischer Elemente und ihrer Verknüpfungen; teilweise können mit Hilfe der aufgeführten Mittel auch implizit in einem Diskurs enthaltene Wissensbestände rekonstruiert werden. Diese sind jedoch vorrangig Gegenstand der Analyse der interdiskursiven Relationen sprachlicher Zeichen.

Interdiskursive Zusammenhänge werden vom Text häufig nicht expliziert, sondern müssen im Verlauf des Rezeptionsprozesses hergestellt werden; daher fokussieren Höhne, Kunz und Radtke in diesem Analyseschritt zunächst das Phänomen der Kohärenz. Der textlinguistische Begriff der Kohärenz bezeichnet den Sinnzusammenhang eines Textes (vgl. Bußmann 1990: 389), den die Rezipierenden anhand von Kohäsionshinweisen innerhalb des Textes, vor allem aber anhand ihres sprachlichen und außersprachlichen Wissens rekonstruieren müssen (vgl. Sandig 2006: 388 f.). Kohärenz basiert demnach ,,auf der präsuppositionalen [...] Struktur des im Diskurs artikulierten Wissens“ (Höhne et al. 2005: 37). Präsuppositionen sind nach Altmayer:

die in Texten vorhandenen und in der Textstruktur nachweisbaren Voraussetzungen [...], mit denen der Text einem potenziellen Rezipienten das Verfügen über bestimmte außersprachliche Wissensbestände unterstellt und die ein potenzieller Rezipient auch anhand seines außersprachlichen Wissens rekonstruieren können muss, um den Text entweder überhaupt als sinnvolle (kohärente) kommunikative Handlung verstehen oder doch zumindest zusätzliche Sinndimensionen des Textes erschließen und zu den im Text enthaltenen Geltungsansprüchen kommunikativ angemessen Stellung nehmen zu können (2004: 198).

Aufgabe der Rezipierenden ist es also, während der Lektüre Wissensbestände, Zusammenhänge und Bedeutungsdimensionen zu erschließen, die vom Text nicht sprachlich expliziert, sondern vorausgesetzt, präsupponiert werden (vgl. Linke, Nussbaumer \& Portmann 2004: 261 ff.). Für eine Textanalyse, deren Gegenstand 
in dem Unterrichtsmedium Lehrwerk besteht, ist an diesem Konzept von besonderem Interesse, dass sich die Wissensbestände der Rezipierenden im Laufe des Rezeptionsprozesses ändern können und sich in vielen Fällen sogar ändern müssen, um Verstehen zu gewährleisten; eine solche Veränderung bedeutet Lernen (vgl. Linke \& Nussbaumer 2000: 442). Die Ermittlung des in den Lehrwerken präsupponierten Wissens lässt damit nicht nur Rückschlüsse auf das bei den Lernenden als bekannt vorausgesetzte Wissen zu, sondern auch auf seine potentielle - intendierte? - Veränderung durch die Lehrbuchtexte.

Eine Möglichkeit zur Ermittlung präsupponierter Wissensbestände in Texten stellt die Argumentationsanalyse nach Toulmin dar (vgl. Höhne et al. 2005: 37), mit deren Hilfe das offengelegt werden soll, „,was ,hinter ${ }^{6}$ der sprachlichen Argumentation steht" (Linke et al. 2004: 273). Die Argumentationstheorie unterscheidet drei Aussagetypen: die Voraussetzung (Datum), die Behauptung (Konklusion) sowie die - kausale - Verknüpfung zwischen beiden, die Schlussregel (Prämisse), die in der Regel nicht verbalisiert wird und samt der präsupponierten Wissensbestände, auf die sie sich bezieht, erschlossen werden muss (vgl. Höhne et al. 2005: 37). Die Bedeutung von Toulmins argumentationsanalytischem Schema für die Lehrwerkanalyse veranschaulicht ein Beispiel von Höhne, das einem Schulbuch für das Fach Sozialkunde entnommen ist (vgl. 2004: 401): „Sie alle [Ausländer] haben - zum Teil sehr große - Schwierigkeiten, im fremden Land Bundesrepublik Deutschland heimisch zu werden. Sie haben Schwierigkeiten mit uns, wir haben Schwierigkeiten mit ihnen“. Das Datum besteht hier - verkürzt - in dem Satz ,Sie leben in einem fremden Land (BRD)“, die Konklusion in dem Satz ,Es gibt Schwierigkeiten mit uns'. Die Schlussregel präsupponiert demnach, ,dass Fremdheit und Heimatlosigkeit per se (!) zu Problemen mit Einheimischen führen“ (Höhne 2004: 401).

Die Untersuchung interdiskursiver Relationen zielt also einerseits auf die Rekonstruktion impliziten Wissens, das in den Texten präsupponiert wird und mit Hilfe des argumentationsanlytischen Verfahrens ermittelt werden soll, und andererseits auf thematische Verknüpfungen eines Diskurses, die durch die Konzepte der Denotation und Konnotation sowie der Topoi und Tropen, darunter besonders Metaphern, Metonymien und Synekdochen, erfasst werden können (vgl. Höhne et al. 2005: 35). Im Hinblick auf das Erkenntnisinteresse des eigenen Projekts ist evident, dass sich über die Ermittlung interdiskursiver Verknüpfungen auch Anschlüsse zu den Gegenständen der Leitfragen, vor allem zum Integrationsbegriff, ergeben können.

Da Lehrbücher außer Texten auch Bilder enthalten, ist im Rahmen einer Lehrwerkanalyse nicht nur eine Text-, sondern auch eine Bildanalyse nötig (vgl. Höhne et al. 2005: 43). Die Analyse von Bildern ist deshalb von besonderer Relevanz, weil sie ähnlich wie das Layout nicht allein zur Illustration dienen, sondern ebenso wie Texte spezifische Wissensbestände präsupponieren (vgl. Altmayer 2004: 243). Bei ihrer Bildanalyse orientieren sich Höhne, Kunz und Radtke vor allem an den Arbeiten von Barthes, der Bilder als sprachlich strukturiert auffasst und damit die theoretische Grundlage schafft, um Bilder analog zu Texten analy- 
sieren zu können (vgl., auch im Folgenden, Höhne et al. 2005: 41 f.). Barthes überträgt sein Modell der denotativen und konnotativen (Schrift-)Zeichen auf Bilder, indem er zwischen nicht kodierten bildlichen und kodierten bildlichen Botschaften unterscheidet. Erstere entsprechen dabei Denotaten, letztere Konnotaten. Zu berücksichtigen ist auch, dass Bilder in Lehrwerken keine isolierte Position einnehmen, sondern in einem funktionalen Zusammenhang mit den Texten stehen, so dass auch eine Untersuchung der Text-Bild-Relationen notwendig ist. Zur näheren Bestimmung der Text-Bild-Relation greifen Höhne, Kunz und Radtke neben Barthes auf Nöth zurück. Während Barthes vor allem die Dependenz der bildlichen Botschaft von der des Textes betont, weist Nöth auf das komplementäre Zusammenwirken von Text und Bild bei der Herstellung von Bedeutung hin und führt weitere Relationen wie Redundanz, Dominanz, Diskrepanz ${ }^{\text {xvii }}$ und Kontradiktion auf (vgl. Nöth 2000: $481 \mathrm{ff}$.).

Insgesamt handelt es sich bei der tDA um ein äußerst elaboriertes Analysemodell, dessen größter Vorteil darin besteht, dass es für die spezifischen Bedürfnisse der Lehrbuchanalyse entwickelt worden und daher unmittelbar auf die Anwendung in der Praxis zugeschnitten ist. Da infolge der primären Orientierung an textlinguistischen Konzepten die Ergebnisse der Analyse und die Interpretationen an den Text zurückgebunden werden können, entspricht die tDA in hohem Maße den Forderungen nach Transparenz und intersubjektiver Nachvollziehbarkeit (vgl. Steinke 2007: 324).

\subsection{Beispielanalyse}

Im letzten Abschnitt des Beitrags soll die tDA exemplarisch angewendet werden. Das Beispiel (Abb. 1 auf der folgenden Seite) entstammt dem Lehrwerk Pluspunkt Deutsch; aus der zweiten Lektion des Bandes 1a wird ein Teil des Abschnitts B 3 behandelt, der die Überschrift „Wir brauchen einen Fernseher!“ trägt (vgl., auch im Folgenden, Pluspunkt Deutsch 1a 2003: 30).

\subsubsection{Diskursformanalyse}

Bei der Betrachtung des Layouts fällt zunächst auf, dass Seite 30 nahezu zweigeteilt ist: In der oberen Hälfte befinden sich zwei Bilder, in der unteren ein Lesetext. Die Bilder sind ebenfalls untereinander angeordnet; sie sind etwa von gleicher Größe und nehmen die Breite der Seite ein. Sie zeigen zwei Zimmer, eine Küche und ein Wohnzimmer, und verweisen damit weitläufig auf das übergeordnete Thema der zweiten Lektion, „Alte Heimat - neue Heimat“ (Pluspunkt Deutsch 1a 2003: 24). Dass an erster Stelle die Küche und an zweiter Stelle das Wohnzimmer abgebildet ist - die gleiche Reihenfolge wird in dem nachfolgenden Text wieder aufgenommen -, hat wohl keine Bedeutung. Hinsichtlich der Markanz ist zu bemerken, dass die Texte unterschiedliche Farben aufweisen: Die Überschrift ist in der Signalfarbe Rot und in einer Schriftgröße von 14 Punkten gedruckt, während für die Aufga- 
benstellung Blau und für den Lesetext Schwarz sowie jeweils eine Schriftgröße von 12 Punkten gewählt wurde. Aufgrund der Farbgebung und der Schriftgröße erweist sich die Überschrift am markantesten; ihr folgen die Aufgabenstellung und zuletzt der Text.

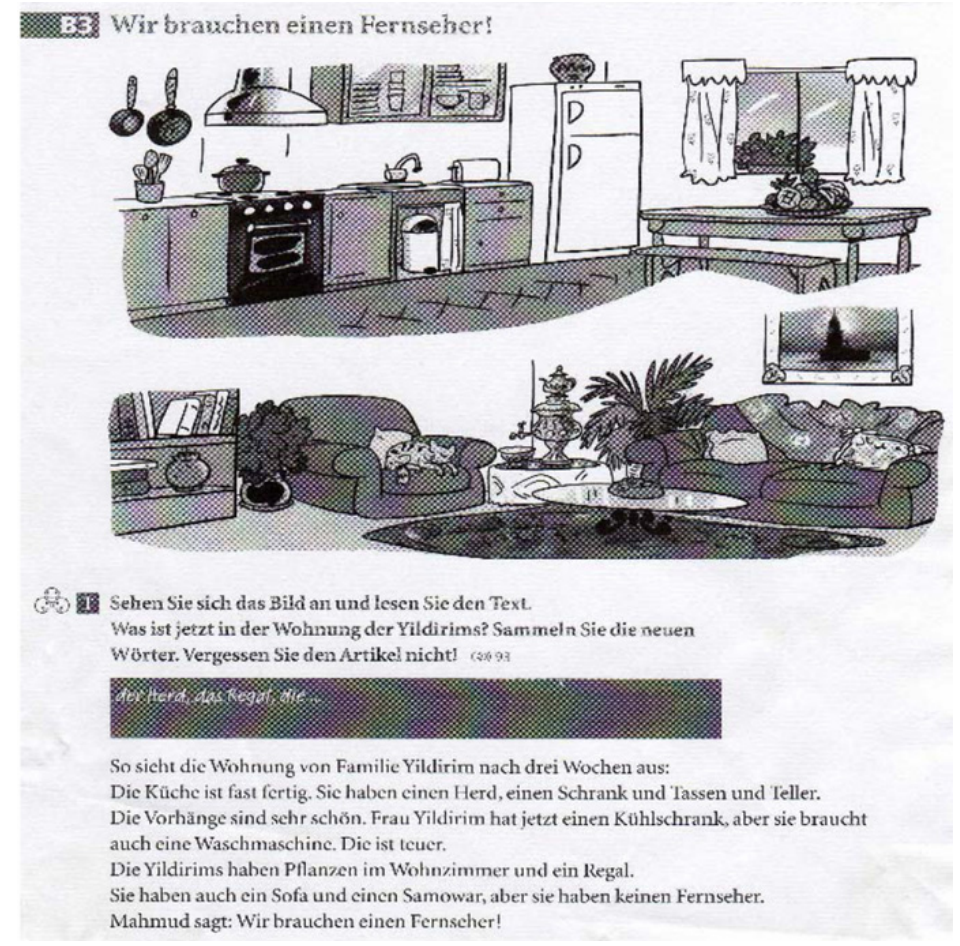

Abb. 1: Pluspunkt Deutsch 1a, Lektion 2, Abschnitt B 3: „Wir brauchen einen Fernseher!“" (S. 30; Ausschnitt).

\subsubsection{Bildanalyse}

Gemäß der Anordnung der Dokumente berücksichtigt die Analyse zunächst die nicht kodierten und dann die kodierten bildlichen Botschaften. Das erste Bild zeigt eine Küche; erkennbar ist dies an Einrichtungsgegenständen wie dem Herd mit Abzughaube, der Spüle und den Kochutensilien. Die Küche erscheint prototypisch, und sie enthält auf den ersten Blick alles, was 'dazugehört'. Dass das zweite Bild ein Wohnzimmer zeigt, ist an Einrichtungsgegenständen wie der Sofagarnitur mit Kissen und Decke, dem Couchtisch mit Gebäckschale, dem Teppich und dem Bücherregal erkennbar. Auch hier rekurriert die Art und Weise der Darstellung im Kontext ,deutscher ${ }^{6}$ Deutungsmuster - auf ein prototypisches Wissen darüber, wie ein Wohnzimmer aussieht und welches Mobiliar es enthält. Durch die Couchgarnitur, die Kissen und die warmen Rottöne, aber auch durch die Pflanzen und 
die schlafende Katze auf dem Sessel wirkt das dargestellte Zimmer sehr gemütlich, was ebenfalls zum prototypischen Wissen über Wohnzimmer gehört, und im Gegensatz zur Küche weniger unpersönlich. Dazu trägt auch der schon aufgrund seiner Größe exponierte Samowar bei, der den Raum individuell erscheinen lässt, allerdings nicht Bestandteil des Wissens über Wohnzimmer im Kontext ,deutscher Deutungsmuster ist und daher den Eindruck des ,Fremdartigen', mindestens aber des Ungewöhnlichen erweckt. Im Kontrast zu dem ,fremdartigen“ Gegenstand, dem Samowar, jedoch in Korrelation zu der Überschrift fällt auf, dass ein Fernseher fehlt, ein Gegenstand, der - spätestens seit Ende der 1960er Jahre - mit einem prototypischen Wohnzimmer assoziiert wird und dessen Besitz deshalb als 'üblich' gelten kann. In dem abgebildeten Raum ist insgesamt die Abwesenheit von etwas ,Typischem‘ oder ,Üblichem‘ und die Anwesenheit von etwas ,Untypischem‘ oder ,Fremden` zu konstatieren; hierdurch entsteht eine binär-chiastische Struktur, die durch das nachfolgende Textdokument aufgenommen und erweitert wird.

Hinsichtlich der Text-Bild-Relation ist festzustellen, dass der Text im Wesentlichen das beschreibt, was auf dem Bild zu sehen ist, und sich ihm gegenüber damit weitgehend redundant ${ }^{\text {xviii }}$ verhält. Allerdings beinhaltet er bildlich nicht darstellbare Zusatzinformationen: Er benennt Gegenstände, die, da sie fehlen, nicht zu sehen sind (Waschmaschine und Fernseher), wertet („Die Vorhänge sind sehr schön.“) und bringt Zeitlichkeit zum Ausdruck (,nach drei Wochen“, ,„jetzt").

\subsubsection{Diskursanalyse der Dokumente (intra-/interdiskursiv)}

Die Überschrift „,Wir brauchen einen Fernseher!“ fungiert in der Terminologie von Höhne, Kunz und Radtke als Eingangssequenz und ist sowohl durch ihre Größe und Farbe als auch durch ihre Positionierung als Überschrift markant hervorgehoben. Syntaktisch handelt es sich um einen Exklamativsatz; sowohl die grammatische Form als auch die typologische Gestaltung verweisen auf die Bedeutung des Besitzes eines Fernsehers. Noch unklar ist an dieser Stelle, wer den Satz ausspricht, wer sich hinter dem „wir“ verbirgt und warum „wir“ einen Fernseher brauchen.

Die Aufgabenstellung ,Sehen Sie sich das Bild an und lesen Sie den Text. Was ist jetzt in der Wohnung der Yildirims?" legt es nahe, das „wir“ mit der Familie Yildirim zu identifizieren. Der bestimmte Artikel hat dabei eine phorische Funktion: Er weist darauf hin, dass die Familie bekannt ist; ${ }^{x i x}$ dem Namen Yildirim lässt sich im Sinne einer impliziten Prädikation entnehmen, dass die Familie wohl türkischer Herkunft ist.xx Eine explizite Prädikation hingegen liegt in der Bezeichnung der dargestellten Wohnung als Wohnung der Familie Yildirim vor. Die Aufgabenstellung trägt somit zur Klärung der Situation bei: Die abgebildeten Zimmer gehören zur Wohnung der Familie Yildirim, die der Überschrift zufolge einen Fernseher braucht. Die temporale Deixis ,jetzt“ bleibt zunächst unbestimmt; aus dem gesamten Satz kann jedoch geschlossen werden, dass sich zum aktuellen Zeitpunkt Dinge in der Wohnung befinden, die es vorher nicht dort gab, dass sich die Familie also neu einrichtet. 
Der anschließende Text spezifiziert die Tempoaldeixis: „jetzt“ ist „nach drei Wochen“, ein Zeitraum der sich, wie die Lernenden aus der ersten Lektion wissen, xxi auf den Einzug in die neue Wohnung bezieht. Im Wesentlichen beschreibt der Text, wie bereits erwähnt, die Bilder.

Von der Küche erfahren die Lernenden, dass sie „fast fertig“ sei. Die Gradpartikel „fast“ verweist auf Unvollständigkeit; da aber zunächst eine Reihe typischer Einrichtungsgegenstände (Herd, Schrank, Geschirr, Vorhänge, Kühlschrank) aufgezählt wird, und auch das Bild den Eindruck einer vollständig eingerichteten Küche vermittelt, erschließt sich nicht unmittelbar, was fehlt. Dies wird erst im folgenden Satz expliziert: „Frau Yildirim hat jetzt einen Kühlschrank, aber sie braucht auch eine Waschmaschine. Die ist teuer.“ Die temporale Deixis ,jetzt" weist darauf hin, dass Frau Yildirim erst zum aktuellen Zeitpunkt - drei Wochen nach dem Einzug - einen Kühlschrank besitzt, d. h. ein Gegenstand, der typischerweise als zentraler Bestandteil jeder Küche gilt, fehlte vorher. Daraus lässt sich schließen, dass die Familie, da sie noch nicht alle ,üblichen' Einrichtungsgegenstände hat, wohl nicht innerhalb Deutschlands umgezogen, sondern nach Deutschland emigriert ist, denn die wenigsten Migrantinnen und Migranten dürften ihre gesamte Einrichtung von einem relativ weit entfernten Herkunftsland wie der Türkei in die BRD transportieren. „Jetzt“ gibt es zwar die wichtigsten Einrichtungsgegenstände; der adversative Konnektor „aber“ hebt jedoch im Gegensatz dazu das Fehlende hervor: die Waschmaschine. Die Betonung des Defizits wirkt relativierend; die positive Wertung, die in der Prädikation „Die Vorhänge sind sehr schön.“ zum Ausdruck kommt, wird damit ebenfalls eingeschränkt. Die asyndetisch mit dem vorangehenden Satz verbundene Feststellung „Die ist teuer.“ bezieht sich, wie der anaphorisch gebrauchte Artikel nahelegt, auf die Waschmaschine. Von den unterschiedlichen Konnektoren, die zwischen asyndetisch verbundenen Sätzen denkbar sind, scheint hier einzig der adversative Konnektor „aber“ angemessen. Demnach steht der Preis der Waschmaschine dem Bedürfnis entgegen, d. h. die Familie ist finanziell nicht in der Lage, bei Bedarf mehrere hundert Euro für eine Waschmaschine auszugeben. Insgesamt erhalten die Rezipierenden einen Eindruck von Defizienz, woraus der Topos vom, armen Ausländer' abgeleitet werden kann: Die Küche ist noch nicht ganz fertig, es gibt keine Waschmaschine, das Geld ist knapp.

Der Abschnitt über das Wohnzimmer ist ähnlich strukturiert wie der über die Küche: Auch hier wird zuerst aufgezählt, was vorhanden ist (Pflanzen, ein Regal, ein Sofa und ein Samowar), um es dann mit dem zu konfrontieren, was fehlt, nämlich dem Fernseher. Der Kontrast wird wiederum mit Hilfe des adversativen Konnektors „aber“ ausgedrückt: „Sie haben auch ein Sofa und einen Samowar, aber keinen Fernseher." Indem diese Feststellung durch eine auktoriale Erzählerinstanz erfolgt und überdies anhand des Bildes nachgeprüft werden kann, erscheint dieser Mangel ,objektiv ${ }^{`}$ und signifikant. Gleichzeitig wird damit die prototypische Vorstellung eines Wohnzimmers aufgerufen, zu der - im Kontext ,deutscher ${ }^{6}$ Deutungsmuster - ein Fernseher, nicht aber ein Samowar gehört. Während also der ,übliche ${ }^{6}$ Einrichtungsgegenstand Fernseher fehlt, ist ein ,unüblicher ${ }^{`}$ vorhanden. 
Die binär-chiastische Opposition zwischen dem anwesenden ,Fremden ${ }^{6}$ und dem abwesenden ,Normalen', die bereits in der Analyse des Bildes in Verbindung mit der Überschrift deutlich wurde, wird durch den Text festgeschrieben und verstärkt. Zusätzlich kann der Familie und dem Samowar als gemeinsames semantisches Merkmal das der Fremdheit zugeordnet werden: Die ,Fremdartigkeit' des Samowars korrespondiert mit der ,Fremdheit` der Familie, die, wie bereits festgestellt wurde, wohl erst vor kurzem in die BRD emigriert, dort also zunächst ebenfalls ,fremd' ist. Demnach fungiert der Samowar als Signifikant des Fremden.xxii Weiterhin führt die Feststellung eines Mangels zu der eines Bedürfnisses: Wenn die Familie keinen Fernseher besitzt, braucht sie einen. Erkannt und ausgesprochen wird dies von Mahmud, dem Sohn der Familie, dem im Text die schon in der Überschrift exponierte Forderung „Wir brauchen einen Fernseher!“ wörtlich und übereinstimmend mit der auktorialen Erzählerinstanz in den Mund gelegt wird. Eben diese Übereinstimmung mit der, objektiven“ Perspektive der Erzählerinstanz weist Mahmuds Forderung als ,richtig' aus. Der Grund für das spezifische Bedürfnis ist auf den ersten Blick nicht ersichtlich, ergibt sich aber aus dem prototypischen Wissen über Wohnzimmer: Familie Yildirim benötigt einen Fernseher, weil das ,dazugehört', ,üblich', ,normal' ist. Gegenüber dem Samowar, der als Signifikant des Fremden erscheint, erscheint der Fernseher damit als Signifikant des Dazugehörens. Diese Deutung ergibt sich zudem aus der binären Opposition zwischen den beiden Gegenständen, die auch auf die mit ihnen verbundenen Signifikate übertragen werden kann. Im Hinblick auf die Zielgruppe des Lehrwerks liegt es nahe, noch weiter zu gehen und den Fernseher in diesem Lehrbuchtext als Sinnbild der Integration bzw. sein Fehlen als Sinnbild der Desintegration zu deuten. Der Familie würde dann als zusätzliches Prädikat das der Integrationsbedürftigkeit zugeschrieben. Vor diesem Hintergrund ist es sogar möglich, den Aufforderungssatz „Wir brauchen einen Fernseher!“ als Aufforderung zur Integration zu lesen. Insgesamt also werden der türkischen Familie allein durch die Feststellung des Besitzes eines Samowars bzw. des Fehlens eines Fernsehers implizit die Prädikate ,fremd

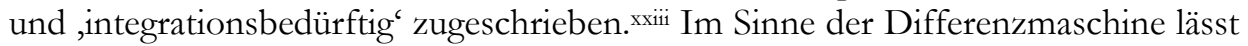
sich dann folgende binäre oder eigentlich dreifache Struktur feststellen: Fernseher - ,deutsches' Wohnzimmer - Zugehörigkeit einerseits, Samowar - ,türkisches ${ }^{6}$ Wohnzimmer - Fremdheit bzw. fehlender Fernseher - , türkisches` Wohnzimmerxxiv - Integrationsbedürftigkeit andererseits.

Zusammenfassend ergibt die Analyse der Bilder und Texte, dass sowohl in der Küche als auch im Wohnzimmer zentrale Gegenstände fehlen, die prototypisch diesen Räumen zugeordnet werden, und dass stattdessen ein Gegenstand vorhanden ist, der im Kontext deutscher Deutungsmuster, fremd' erscheint. Weitere notwendige Haushaltsgeräte besitzt die Familie erst seit kurzem bzw. stehen ihrer Anschaffung hohe Kosten entgegen. Mit Hilfe der anwesenden ,fremden' und der abwesenden ,üblichen' Gegenstände werden der türkischen Familie die Prädikate ,arm‘, ,fremd‘ und ,integrationsbedürftig' beigelegt. ${ }^{x v}$ Damit wird offensichtlich, wie ein Lehrwerk für den Integrationskurs diejenigen zu Fremden macht, die dem 
Anspruch nach mit seiner Hilfe eigentlich integriert werden sollen. Ob diese Zuschreibungen im Hinblick auf die Konstruktion von Wirklichkeit homogenisierend wirken, kann die Analyse singulärer Diskursdokumente allerdings nicht verbindlich belegen; zuverlässige Aussagen erlaubt erst die vergleichende Betrachtung weiterer Texte und Bilder.

Wie die Beispielanalyse verdeutlicht, beschränkt sich das Projekt im Sinne von Höhne, Kunz und Radtke auf die Analyse des Zustandekommens und der Struktur von Lehrbuchdiskursen und damit auf „Diskursaufklärung“ (Höhne et al. 2005: 28). Intendiert ist weder eine empirische Wirkungsforschung noch eine Rekonstruktion der Aussageabsicht der einzelnen Lehrwerkautorinnen und -autoren, sondern vielmehr die Anregung zu einer kritischen Reflexion landeskundlicher Inhalte in Lehrmaterialien. Da die thematische Diskursanalyse aufgrund ihrer engen Orientierung an den Text- und Bilddokumenten zuverlässige und intersubjektiv nachvollziehbare Ergebnisse ermöglicht, kann sie einer solchen Kritik eine solide Grundlage bieten.

\section{Literatur}

Abendroth-Timmer, Dagmar (1998): Der Blick auf das andere Land. Ein Vergleich der Perspektiven in Deutsch-, Französisch- und Russischlehrwerken. Tübingen: Narr.

Altmayer, Claus (2004): Kultur als Hypertext. Zu Theorie und Praxis der Kulturwissenschaft im Fach Deutsch als Fremdsprache. München: Iudicium.

Altmayer, Claus (2006): ,Kulturelle Deutungsmuster ${ }^{6}$ als Lerngegenstand. Zur kulturwissenschaftlichen Transformation der Landeskunde. In: Fremdsprachen Lebren und Lernen 35, 44-59.

Ammer, Reinhard (1988): Das Deutschlandbild in den Lehrwerken für Deutsch als Fremdsprache. München: Iudicium.

Barkowski, Hans et al. (1979): Kriterien zur Beurteilung von Lehrwerken für den Deutschunterricht mit ausländischen Arbeitern. In: Deutsch lernen 4(4), 3-10.

Barkowski, Hans et al. (31986): Deutsch für ausländische Arbeiter. Gutachten zu ausgewäblten Lebrwerken. Mainz: Werkmeister.

Bung, Peter (1977): Systematische Lehrwerkanalyse. Untersuchungen zum Einsatz von Contentanalysen für die Lehrwerkforschung und Lehrwerkkeritik. Kastellaun: Henn.

Bußmann, Hadumod (21990): Lexikon der Sprachwissenschaft. Stuttgart: Kröner.

Engel, Ulrich et al. (1977 u. 1979): Mannheimer Gutachten zu ausgewäblten Lehrwerken Deutsch als Fremdsprache. 2 Bde. Heidelberg: Groos.

Funk, Hermann (1994): Arbeitsfragen zur Lehrwerkanalyse. In: Kast, Bernd \& Neuner, Gerhard (Hrsg.) (1994): Zur Analyse, Begutachtung und Entwicklung von Lebrwerken für den fremdsprachlichen Deutschunterricht. Berlin: Langenscheidt, 105-111. 
Gadatsch, Michael et al. (1990): Fragen zur Beurteilung von Lehrwerken in Kursen, die vom Sprachverband - Deutsch für ausländische Arbeitnehmer e. V. gefördert werden. In: Deutsch lernen 15(1), 48-69.

Höhne, Thomas (2004): Die thematische Diskursanalyse - dargestellt am Beispiel von Schulbüchern. In: Keller, Reiner et al. (Hrsg.) (2004): Handbuch Sozialwissenschaftliche Diskursanalyse. Bd. 2: Forschungspraxis. Wiesbaden: Verlag für Sozialwissenschaften, 389-419.

Höhne, Thomas; Kunz, Thomas \& Radtke, Frank-Olaf (1999): Zwischenbericht: Bilder von Fremden. Formen der Migrantendarstellung als der, anderen Kultur' in deutschen Schulbüchern von 1981-1997. Frankfurt a. M.: Johann Wolfgang Goethe-Universität.

Höhne, Thomas; Kunz, Thomas \& Radtke, Frank-Olaf (2005): Bilder von Fremden. Was unsere Kinder aus Schulbüchern über Migranten lernen sollen. Frankfurt a. M.: Johann Wolfgang Goethe-Universität.

Istanbul Metropolitan Municipality: Homepage der Stadt. [http://www.ibb.gov.tr.; 20.09.2008].

Kast, Bernd \& Neuner, Gerhard (Hrsg.) (1994): Zur Analyse, Begutachtung und Entwicklung von Lebrwerken für den fremdsprachlichen Deutschunterricht. Berlin: Langenscheidt.

Keller, Reiner (2004): Diskursforschung. Eine Einfübrung für SozialwissenschaftlerInnen. Wiesbaden: Verlag für Sozialwissenschaften.

Kress, Gunther \& Van Leeuwen, Theo (1995): Critical Layout Analysis. In: Internationale Schulbuchforschung 17(1), 25-43.

Krüger, Georg et al. (2003): Pluspunkt Deutsch 1a. Der Integrationskurs Deutsch als Zweitsprache. Kursbuch. Berlin: Cornelsen.

Krüger, Georg et al. (2003): Pluspunkt Deutsch 1b. Der Integrationskurs Deutsch als Zweitsprache. Kursbuch. Berlin: Cornelsen.

Krüger-Potratz, Marianne (2005): Interkulturelle Bildung. Eine Einführung. Münster: Waxmann.

Krumm, Hans-Jürgen (1994): Zur Situation der Lehrwerkkritik und Lehrwerkforschung Deutsch als Fremdsprache. In: Kast, Bernd \& Neuner, Gerhard (Hrsg.) (1994): Zur Analyse, Begutachtung und Entwicklung von Lehrwerken für den fremdsprachlichen Deutschunterricht. Berlin: Langenscheidt, 23-29.

Krumm, Hans-Jürgen \& Ohms-Duszenko, Maren (2001): Lehrwerkproduktion, Lehrwerkanalyse, Lehrwerkkritik. In: Helbig, Gerhard et al. (Hrsg.) (2001): Deutsch als Fremdsprache. Ein internationales Handbuch. 2. Halbband. Berlin: De Gruyter, 1029-1041.

Linke, Angelika \& Nussbaumer, Markus (2000): Konzepte des Impliziten: Präsuppositionen und Implikaturen. In: Brinker, Klaus et al. (Hrsg.) (2000): Text-und Gesprächslinguistik. Ein internationales Handbuch zeitgenössischer Forschung. Berlin: de Gruyter, 435-448.

Linke, Angelika; Nussbaumer, Markus \& Portmann, Paul R. (52004): Studienbuch Linguistik. Tübingen: Niemeyer. 
Maijala, Minna (2004): Deutschland von außen gesehen. Geschichtliche Inhalte in Deutschlehrbüchern ausgewählter europäischer Länder. Frankfurt a. M.: Peter Lang.

Neuner, Gerhard (1979): Lehrwerkanalyse und -kritik als Aufgabenfeld der Fremdsprachendidaktik - zur Entwicklung seit 1945 und zum gegenwärtigen Stand. In: Neuner, Gerhard (Hrsg.) (1979): Zur Analyse fremdsprachlicher Lebrwerke. Frankfurt a. M.: Peter Lang, 5-39.

Neuner, Gerhard (1994): Lehrwerkforschung - Lehrwerkkritik. In: Kast, Bernd \& Neuner, Gerhard (Hrsg.) (1994): Zur Analyse, Begutachtung und Entwicklung von Lebrwerken für den fremdsprachlichen Deutschunterricht. Berlin: Langenscheidt, 8-22.

Neuner, Gerhard (1994): Lehrwerkbegutachtung für die Praxis: die Lehrstoff-, Lehrer- und Lernerperspektive. In: Kast, Bernd \& Neuner, Gerhard (Hrsg.) (1994): Zur Analyse, Begutachtung und Entwicklung von Lehrwerken für den fremdsprachlichen Deutschunterricht. Berlin: Langenscheidt, 111-118.

Nöth, Winfried (2000), Handbuch der Semiotik. Stuttgart: Metzler.

Sandig, Barbara (2006), Textstilistik des Deutschen. Berlin: De Gruyter.

Schröder, Martin Z. (2001): Krach um den Couchtisch. Berliner Zeitung, 30.10.2001, 11. [http://www.berlinonline.de/berliner-zeitung; 20.09.2008].

Steinke, Ines (52007): Gütekriterien qualitativer Forschung. In: Flick, Uwe; Kardoff, Ernst \& von Steinke, Ines (Hrsg.) (2007): Qualitative Forschung. Ein Handbuch. Reinbek: Rowohlt, 319-331.

Thimme, Christian (1996): Geschichte in Lehrwerken. Deutsch als Fremdsprache und Frañösisch als Fremdsprache für Erwachsene. Ein deutsch-französischer Lebrbuchvergleich. Baltmannsweiler: Schneider Verlag Hohengehren.

Walter, Andreas (2002): Ein Buch über Tee. [http://www.teebuch.de/teebuch.pdf; 20.09.2008].

i Lehrwerke für Integrationskurse umfassen Lehrwerke für den Sprach- und Orientierungskurs. Gegenstand der Untersuchung sind die Lehrwerke für Deutsch als Zweitsprache Berliner Plat\%, Mit uns leben, Pluspunkt Deutsch und Schritte sowie die Lehrwerke für den Orientierungskurs 30 Stunden Deutschland, Miteinander leben, Orientierungskurs: Gescbicbte, Institutionen, Leben in Deutscbland, Pluspunkt Deutsch - Der Orientierungskurs DaZ und Zur Orientierung - Deutscbland in 30 Stunden. Bei der Analyse werden sowohl die Kurs- als auch die Arbeitsbücher einschließlich der Hörtexte berücksichtigt. Kriterien für die Auswahl der Lehrwerke waren unter anderem das Selbstverständnis als Inlands- oder Zweitsprachen-Lehrwerk, die Zulassung durch das Bundesamt für Migration und Flüchtlinge und eine - relative - Aktualität.

ii Es ist davon auszugehen, dass kulturell homogene Gesellschaften im strengen Sinne nie existiert haben; Homogenität ist daher immer nur relativ zu denken.

iii Dass Globalisierungsprozesse wieder andere Formen von Homogenität hervorbringen können, ist ein Problem, auf das in diesem Beitrag nicht weiter eingegangen werden kann.

iv „Diskurs‘ wird hier mit Höhne, Kunz und Radtke als „thematisch gebundene, sprachlichsemiotische Einheit“ (2005: 30) verstanden. 
v Als Vergleichstexte wurden diejenigen Texte ausgewählt, die einen direkten Bezug zu den Integrationskursen aufweisen oder auf die sich die Lehrwerke explizit beziehen: das Zuwanderungsgesetz. der Bundesregierung, die Integrationskursverordnung des Bundesinnenministeriums, das Konzept für einen bundesweiten Integrationskurs, das Rabmencurriculum für Integrationskurse Deutsch als Zweitsprache und das Curriculum für einen bundesweiten Orientierungskurs des Bundesamts für Migration und Flüchtlinge sowie der Gemeinsame europäische Referenærahmen für das Lebren und Lernen fremder Sprachen des Europarats. Zusätzlich werden die Lehrwerke selbst - soweit das möglich ist - zu ihren pädagogischen und landeskundedidaktischen Grundlagen befragt; diese sind vor allem Vorworten, Lehrerhandbüchern und Verlagspublikationen zu entnehmen.

vi Bungs Erkenntnisinteresse richtet sich unter anderem auf die Frage, ob in Englisch-Lehrwerken für die Haupt- bzw. Oberschule ein ausgewogenes Bild der Gesellschaft gezeichnet wird. Ein ausgewogenes Bild liegt nach Bung dann vor, wenn die Gesellschaftsbereiche Politik, Wirtschaft, Familie und Kultur in Texten und Bildern gleichermaßen (d. h. jeweils zu 20 bis 30 \%) berücksichtigt sind (vgl. 1977: 121f.).

vii In jüngerer Zeit treten allerdings Mischformen auf: So geht etwa Maijala, die historische Inhalte in Lehrwerken für Deutsch als Fremdsprache untersucht, zunächst empirisch-quantitativ vor, indem sie die Häufigkeit der Thematisierung historischer Inhalte misst (vgl. 2004: 57f.). AnschlieBend wertet sie die Ergebnisse vor dem Hintergrund von Leitfragen zu Inhalt, Didaktik und Darstellung aus (vgl. 2004: 58ff.).

viii Auch Bung fordert daher eine Integration von empirisch-quantitativen und hermeneutischen Methoden (vgl. 1977: 81f.).

ix Beispiele für Kataloge sind das Mannheimer Gutachten (1977 und 1979), wodurch sich diese Art der Lehrwerkanalyse etabliert hat, die zielgruppenspezifischen Kataloge des Sprachverbandes Deutsch für ausländische Arbeitnehmer (vgl. Barkowski 1979 und 1986, Gadatsch et al. 1990) und der überaus elaborierte landeskundliche Kriterienkatalog von Ammer (1988).

x Abendroth-Timmer etwa untersucht vor dem Hintergrund der Diskussion um das interkulturelle Lernen und der Frage nach der wechselseitigen Eigen- und Fremdperspektive die unterschiedlichen Perspektiven, die in Lehrwerken deutlich werden, und folgt dabei der an Gadamer orientierten Methode der ,hermeneutischen Inhaltsanalyse' (vgl. 1998: 178f.).

xi Die Kurzform tDA wurde von Höhne, Kunz und Radtke übernommen (vgl. 2005: 28).

xii Das Interesse an der Erschließung von implizitem bzw. präsupponiertem Wissen in Diskursen teilt die tDA mit der Kulturwissenschaft des Faches Deutsch als Fremdsprache (vgl. Altmayer 2004: 198). Auch deshalb lag die Wahl dieser Methode nahe.

xiii Die Darstellung des Ablaufschemas der tDA orientiert sich vor allem an der letzten Version aus dem Jahr 2005 (vgl. Höhne et al. 2005: 33ff.). Höhne, Kunz und Radtke betonen, dass die einzelnen Instrumente nicht in jedem Fall zur Anwendung gelangen, sondern dass ,gegenstands- und dokumentenspezifisch [auf sie] zurückgegriffen“ (2005: 35) wird.

xiv Die Punkte 2 und 3 beziehen sich ausschließlich auf die Textdokumente. 'Text' wird hier alltagssprachlich als verbalsprachlicher Text verstanden.

xv In den folgenden Abschnitten werden die einzelnen Analyseinstrumente und Untersuchungsgegenstände kursiv hervorgehoben.

xvi Das implizite Diskurswissen lässt sich teilweise mit Hilfe der Kohäsionsmittel erschließen; das Ergänzen lediglich implizit im Text vorhandenen Wissens beschreibt aber vor allem einen Prozess, der für die Herstellung von Kohärenz konstitutiv ist (vgl. den Abschnitt zu den interdiskursiven Relationen). Die Grenzen sind hier häufig fließend.

xvii Von Diskrepanz ist die Rede, wenn Text und Bild zusammenhanglos nebeneinander stehen (vgl. Nöth 2000: 484). 
xviii Die Redundanz ist selbstverständlich didaktischen Gründen geschuldet.

xix Die Familie wurde bereits in der ersten Lektion eingeführt (vgl. Pluspunkt Deutsch 1a 2003: 12 f.).

xx Das Bild an der Wohnzimmerwand könnte den Jungfrauenturm, der sich auf einer kleinen Felseninsel vor Istanbul befindet, darstellen (vgl. Istanbul Metropolitan Municipality: Homepage der Stadt: http://www1.ibb.gov.tr; 20.09.2008) und damit diese Deutung unterstreichen.

xxi Herr Yildirim ist hier beim Tragen eines Umzugskartons abgebildet (vgl. Pluspunkt Deutsch 1a 2003: 12).

xxii Ein Beleg für die Kodierung des Samowars als Signifikant des Fremden findet sich in Abschnitt B 2 (vgl. Pluspunkt Deutsch 1a 2003: 29): Hier muss Mahmud, der ebenfalls schon in Lektion 1 eingeführte Sohn der Familie Yildirim (vgl. Pluspunkt Deutsch 1a 2003: 13), einem anderen - deutschen? - Jungen erklären, dass es sich bei einem Samowar um einen Teebereiter und nicht um eine Lampe handelt, wie dieser glaubt. Es kann gefolgert werden: Ein Samowar ist ein Gegenstand, der den Mitgliedern der Familie Yildirim vertraut, anderen - Deutschen? - jedoch fremd ist. Das Motiv des Samowars bzw. der Fremdheit spielt damit in zwei Abschnitten über die Wohnung der Familie Yildirim eine Rolle. Für die Analyse ist es hingegen irrelevant, dass der Samowar außer in der Türkei auch in Russland und dem Nahen Osten verwendet wird (vgl. Walter 2002: 64 f.); von Bedeutung ist hier allein seine Funktion als Sinnbild des Fremden.

xxiii Eine weitere implizite Prädikation ergibt sich möglicherweise aus der Funktion des Fernsehers als modernes Informationsmedium: Wer wie die Familie Yildirim keinen besitzt, hat keinen Zugang zu aktuellen Informationen, ist mithin nicht ,auf der Höhe der Zeit'. Das Fehlen des Fernsehers würde demnach den Topos der, rückständigen (türkischen) Migrantinnen und Migranten` evozieren. Vor dem Hintergrund, dass aktuelle Informationen auch über andere Medien zugänglich sind, ist diese Deutung allerdings nicht zwingend.

xxiv In diesem Zusammenhang ist ein Interview mit der Soziologin und Ethnologin Ayse Çaglar interessant, in dem sie u. a. auf die zentrale Position des Fernsehers in den Wohnzimmern vieler türkischer Familien hinweist (vgl. Schröder 2001: 11). Im gleichen Interview wird auch die seit etwa zwanzig Jahren zunehmende Haustierhaltung in türkischen Haushalten thematisiert, so dass die Katze, die im Wohnzimmer der Familie Yildirim auf dem Sessel liegt, nicht zwangsläufig spezifische Deutungen (etwa im Hinblick auf Integration) evoziert.

xxv Wie in einer späteren Lektion deutlich wird, ist die Familie Yildirim nicht erst vor kurzem in die BRD immigriert, sondern lebt schon länger dort (vgl. Pluspunkt Deutsch 1b 2003: 42f.). Demzufolge ist anzunehmen, dass sie auch in ihrer vorigen Wohnung weder einen Kühlschrank noch eine Waschmaschine noch einen Fernseher besessen hat. Die Zuschreibung der genannten Attribute verfestigt sich damit. 



\title{
Kulturwissenschaftliche Diskursanalyse - ein Praxisbericht
}

\author{
Paul Voerkel (Belém)
}

\begin{abstract}
In der Disziplin Deutsch als Fremdsprache herrscht heute weitgehend Konsens darüber, dass Fragen zu kulturellem Lernen sowohl in der Forschung als auch im Fremdsprachenunterricht eine wachsende Rolle spielen. Entsprechend wird nach adäquaten Verfahren gesucht, mit denen die diesbezüglichen Fragen und Bedürfnisse von Fremdsprachenlernern erfasst und im Unterricht berücksichtigt werden können. Dieser Beitrag beschreibt die Erfahrungen, die der Verfasser beim Entwickeln seiner Magisterarbeit mit der kulturellen Diskursanalyse gesammelt hat. Im Zentrum stand dabei die Frage, inwieweit mit der Beschreibung von ,kulturellen Deutungsmustern' das kulturelle Lernen im Fremdsprachenunterricht, insbesondere in Deutsch als Fremdsprache $(\mathrm{DaF})$, gefördert werden kann. Auf den folgenden Seiten sollen vor allem die Herausforderungen, Schwierigkeiten und Chancen aufgezeigt werden, die bei der Auseinandersetzung mit dem Verfahren einer solchen Diskursanalyse auftreten können.
\end{abstract}

\section{Einleitung}

In der Wissenschaft sind heutzutage Verfahren der Diskursanalyse für einen kritischen Nachvollzug des menschlichen Denkens nicht mehr wegzudenken. Auch in der im Entstehen begriffenen Teildisziplin Kulturwissenschaft DaF finden Diskursanalysen zunehmend Verwendung. Dieser neuen Teildisziplin geht es vor allem darum, anhand von Analysen die Konstruktion des menschlichen Denkens und vor allem die Strukturen von Kommunikation sichtbar zu machen, um dieses Wissen für die Unterrichtspraxis von Deutsch als Fremdsprache zu nutzen (vgl. Altmayer 2006: 54).

Mit Konzepten wie der Herausarbeitung und Interpretation von ,kulturellen Deutungsmustern' (u. a. Altmayer 2002: 14 ff.) liegt ein sich stetig erweiterndes, wissenschaftlich fundiertes Instrumentarium vor, um aus der Perspektive der Kulturwissenschaft DaF auch Verfahren einer Diskursanalyse zum Erkenntnisgewinn nutzen zu können (Altmayer 2007: 267). Ein Verfahren für die praktische Anwendung hat sich hingegen noch nicht durchgesetzt.

Auf den folgenden Seiten wird der Versuch unternommen, die vorhandenen theoretischen Konzepte sowohl aus Deutsch als Fremdsprache als auch aus Nachbardisziplinen unter kulturwissenschaftlichen Fragestellungen zusammenzuführen und anhand einer umfassenden Analyse auf ihre Relevanz für die DaF-Praxis hin 
zu überprüfen. Auf diese Weise soll gezeigt werden, wie ein Verfahren der Diskursanalyse in der Praxis angewendet werden kann; vor allem aber geht es um den Erkenntnisgewinn, der anhand einer solchen Analyse auch im Bereich der Kulturwissenschaft möglich ist.

Der vorliegende Artikel umfasst mehrere Teile. Im ersten Teil ist dargestellt, auf welcher Basis sich die Fragestellungen einer kulturwissenschaftlichen Diskursanalyse konstituieren, um dem Leser das Verständnis des Analyseverfahrens zu erleichtern, welches auch der Magisterarbeit zugrunde lag, auf welcher der Artikel beruht. Dieses Verfahren wird in einem eigenen Abschnitt offen gelegt, der zum zweiten Teil überleitet. Im zweiten Teil werden neben einem konkreten Beispiel und den Ergebnissen der Diskursanalyse vier wesentliche Probleme diskutiert, auf die ich im Laufe der Analyse gestoßen bin und welche ich versucht habe, mithilfe des beschriebenen Verfahrens zu lösen.

- Probleme der Kodierung bei einer Diskursanalyse

- Eingrenzung und Gebrauch von ,kulturellen Deutungsmustern“

- Potenzial einer Diskursanalyse zur Beschreibung der Tiefenstruktur von Diskursen

- Konkrete Anwendung von Erkenntnissen aus einer Diskursanalyse.

Wichtig ist es mir dabei, zu zeigen, an welchen Stellen nach wie vor Fragen offen geblieben sind - aus diesem Grund sind weniger fertige Antworten zu erwarten als vielmehr Vorschläge, die es zu hinterfragen gilt. Nach dem Hinweis auf Herausforderungen und mögliche Schwierigkeiten bei dem verwendeten Verfahren wird im abschließenden Ausblick danach gefragt, wo die Möglichkeiten und Grenzen für die praktische Anwendung einer kulturwissenschaftlichen Diskursanalyse zu sehen sind.

\section{Theoretischer Hintergrund der Arbeit}

Die eingangs erwähnte Magisterarbeit trägt den Titel „,Kulturelle Deutungsmuster“ im Vergleich - eine Diskursanalyse über Fußball"“ und ist in dem Jahr entstanden, in dem in der Bundesrepublik Deutschland die Fußball-Weltmeisterschaft stattfand - doch ist dieses Großereignis nicht der einzige Grund, warum Texte über Fußball das Korpus der Arbeit bilden. Im Bereich Deutsch als Fremdsprache ist der Zusammenhang zwischen Sprachlernen und kulturellem Lernen inzwischen unbestritten (Altmayer 2005: 154). Fußball wiederum wird oft als kulturelles Phänomen gedeutet, das tief in der Gesellschaft verwurzelt ist (Schiffer 2004: 1; Silva 2006: 3), Kultur prägt (u. a. Pornschlegel 2002: 103; Martínez 2002: 26f.; Gebauer 2000: 150) oder diese gar konstituiert (Adelmann, Parr \& Schwarz 2003: 7). Fußball wird in diesem Sinne als Element des kulturellen Denkens einer Nation begriffen (Wagner 2004: 146). So darf es nicht verwundern, dass es inzwischen salonfähig geworden ist, über Fußball zu sprechen, zu schreiben und zu forschen (Brussig 2003: 
175) - auch aus der DaF-Perspektive heraus. Die stetig wachsende wissenschaftliche Auseinandersetzung mit diesem Lebensbereich unterstreicht noch einmal die Stellung von Fußball als gesellschaftliches Phänomen (Hütig \& Marx 2004: 12). Nicht zuletzt aus diesem Grund kann man sich fragen, warum Fußball, oder allgemeiner gefasst: Sport, in traditionellen Lehrbüchern oder in Curricula zum Fremdsprachenlernen ( $\mathrm{DaF}$ eingeschlossen) kaum zu finden ist. ${ }^{\mathrm{i}}$ Diese Frage wird am Ende des Artikels noch einmal aufgegriffen.

Was aber ist nun unter einer „Kulturwissenschaft $\mathrm{DaF}^{\text {“ }}$ zu verstehen? Wenn auch die Grenzen der im Entstehen begriffenen Teildisziplin des Faches Deutsch als Fremdsprache bisher nicht genau abgesteckt und viele Fragen noch offen sind, so lassen sich unter kulturwissenschaftlicher Perspektive dennoch zwei Aufgaben besonders herausstellen: Erstens werden Fragen aus der Lehr- und Lernpraxis DaF aufgegriffen, die von der bisher institutionalisierten Landeskunde nicht zufriedenstellend beantwortet werden konnten (Altmayer 2006: 50), und zweitens Methoden und Vorgehensweisen angewendet, die fundierte Auseinandersetzungen mit kulturellen Fragestellungen ermöglichen und die Erkenntnisse aus den Forschungsprozessen wissenschaftlich fundiert an die (Unterrichts-)Praxis DaF zurückgeben können (Altmayer 2005: 155). Der Anspruch einer Kulturwissenschaft DaF bettet sich also wesentlich in die (spätestens in der Praxis stets wieder aktuelle) Diskussion um Inhalte des $\mathrm{DaF}$ - und insbesondere des Landeskundeunterrichts ein.

Kulturelles Lernen wird, verstanden entweder als allgemeiner Prozess oder als spezifisches Modell, inzwischen als genuiner Teil des Fremdsprachenlernens akzeptiert (u. a. Altmayer 2005: 154), wobei die wissenschaftlichen Erklärungsversuche der wechselseitigen Beziehung zwischen Sprache und Kultur bisher wenig zufriedenstellend ausfielen. Neue Erkenntnisse der Neuropsychologie können inzwischen nicht nur Aussagen zu den Arbeitsprozessen des Gehirns beim Sprachenlernen treffen, sondern sehr genau auch die Funktion bestimmter Neurone bei komplexen Kommunikationsprozessen erklären (vgl. Bauer 2005). Die u. a. von Joachim Bauer beschriebenen neuronalen Prozesse stützen ihrerseits auf teilweise verblüffende Weise Theorien zu Spracherwerbsprozessen und zu kulturellem Lernen, die seit Jahren auch im Bereich Deutsch als Fremdsprache diskutiert werden.

Aus den wissenschaftlichen Arbeiten Claus Altmayers waren neben Grundaussagen zu Textverständnis und -rezeption zwei wesentliche Gedankengänge wichtig, um dem in der Magisterarbeit angewandten Verfahren einer kulturwissenschaftlichen Diskursanalyse einen Rahmen zu geben. Zum einen betrifft es die Frage, mithilfe welcher Prozesse Menschen (und insbesondere Fremdsprachenlerner) ihre Umwelt deuten und konstruieren - das Beschreibungsmittel hierzu sind die ,kulturellen Deutungsmuster', auf die weiter unten noch explizit eingegangen wird (für eine umfassendere Definition sei auf Altmayer (2002: 16f.) verwiesen). Zum andern geht es darum, inwieweit Fremdsprachenlerner einen Diskurs in ihrer Zielsprache möglichst bewusst nachvollziehen können müssen, um in einen Lernprozess einzutreten. Dabei meint Altmayer nicht nur, dass es „eine zentrale Aufgabe der Kulturwissenschaft DaF [ist], die Lerner zur Teilnahme an einem Diskurs zu 
befähigen“ (Altmayer 2006: 54), sondern dass zudem der ,,verstehende Nachvollzug von Diskursen eine Möglichkeit dar[stellt], ,Kultur zu beschreiben“ (Altmayer 2003: 125). Auf den Aspekt der Partizipation wird am Ende des Beitrags noch einmal zurückzukommen sein, wenn nach praktischen Implikationen der Diskursanalyse für die Unterrichtspraxis DaF gefragt wird. Die Notwendigkeit einer weiterführenden Analysearbeit, welche die den Diskursen zugrunde liegenden Deutungsmuster herausarbeitet und beschreibt, wird immer wieder betont (bspw. Altmayer 2006: 57), um Lernern das nötige Material für den eigenen Lernprozess zugänglich zu machen. An dieser Stelle setzt die Magisterarbeit an, die mit einer umfassenden Textanalyse aufzeigen möchte, auf welche Weise die dem heterogenen Fußballdiskurs zugrunde liegenden Verstehensmuster zur Anregung eines ,kulturellen Lernprozesses' bei Fremdsprachenlernern beitragen können.

Um gesellschaftliche Diskurse, sei es über Fußball oder ein anderes Thema, mit einer sinnvollen Tiefe beschreiben zu können, ist ein mehrstufiges Verfahren nötig, das im folgenden Kapitel skizziert wird, wobei es vornehmlich um die einzelnen Analyseschritte geht.

\section{Das Analyseverfahren}

Wie generell bei der Arbeit mit Texten stellt sich bei einer Diskursanalyse das Problem, dass die Texte des Korpus, welche die Grundlage für die Forschung bilden, in den seltensten Fällen verfasst wurden, um analysiert zu werden. Die eigentliche Arbeit besteht entsprechend darin, das Material so zu strukturieren (und meist auch zu komprimieren), dass eine sinnvolle Interpretation möglich wird. Kurz: Das Ziel ist es, von einem geschlossenen Text zu den ,kulturellen Deutungsmustern' vordringen. Im Laufe der Analyse treten dabei immer wieder mindestens zwei Fragen auf, die schlüssige Antworten fordern:

a) Wie lese ich die Deutungsmuster aus einem Text heraus?

b) Wie begründe ich meine Deutung stichhaltig und nachvollziehbar?

Bei der Erarbeitung meiner Magisterarbeit versuchte ich diesen Fragen zu begegnen, indem ich möglichst ausführlich und nachvollziehbar die einzelnen Stufen der Analyse offen legte. Die Arbeit beruhte also auf mehreren Analyseschritten, die zwar teilweise mit anderen Begriffen bezeichnet werden, im Wesentlichen aber einem Schema der Sequenzanalyse folgen wie sie Claus Altmayer in seinem Artikel zur Kulturwissenschaftlichen Diskursanalyse (vgl. Altmayer 2007: 272ff.) exemplarisch durchführt.

Das Korpus besteht aus 75 Online-Pressetexten sechs verschiedener Tageszeitungen aus Deutschland, Argentinien und Brasilien, die sich alle mehr oder weniger direkt auf Fußballspiele der WM 2006 in Deutschland bezogen ii. Auf der Grundlage dieses Materials erschien das folgende Vorgehen am ehesten Erfolg versprechend: 
1. Bezug nehmend auf Reiner Keller (2003: 283) wurde in den zur Verfügung stehenden Texten nach Unterschieden und Gemeinsamkeiten gesucht. Hier ging es um eine erste Kategorisierung nach inhaltlichen Kriterien. Bereits in dieser Phase konnte eine Einteilung vorgenommen werden in Texte, die sich a) auf das Spielgeschehen konzentrieren und b) ein Spiel zum Anlass nehmen, um das Umfeld (im weitesten Sinne) des Fußballs zu beschreiben.

2. In einem zweiten Schritt wurden vier Basistexteiii genauer danach befragt, welche potenziellen Deutungsmuster hier dem Fußballdiskurs zugrunde liegen. Nachdem im ersten Schritt nach dem was des Diskurses gefragt wurde, geht es in diesem Schritt um das wie. Die Ergebnisse dieser Mikroanalyse wurden im Anschluss zusammengefasst, indem untersucht wurde, welche ,kulturellen Deutungsmuster' sich wiederholen und in welchem Kontext dies geschieht.

3. Der Idee einer ,zirkulären Strategie‘ von Harald Witt (2001: 15) folgend, laut der eine bestimmte Aufeinanderfolge von Forschungsschritten mehrmals durchlaufen wird um Ergebnisse in der qualitativen Sozialforschung zu optimieren, erfolgte in einem dritten Schritt die interne Gegenüberstellung der Basistexte mit weiteren deutschsprachigen Zeitungstexten. Hier lag die Aufgabe darin, die bisher gefundenen ,kulturellen Deutungsmuster ${ }^{6}$ aufmerksam zu verfolgen und zu schauen, inwieweit sie den Fußballdiskurs des untersuchten Zeitraums tatsächlich repräsentieren. Auf diese Weise wurde ein Kategoriensystem erstellt, das alle wesentlichen Deutungsmuster erfasste. Folgende Hauptkategorien, unter denen einzelne ,kulturelle Deutungsmuster' zusammengefasst wurden, konnten entsprechend herausgearbeitet werden: „deutsche Fußball-Nationalmannschaft“, „,die Deutschen“, „Fußballfans“, „Personen der Geschichte“, „aktuelle Spieler und Personen“, „Presse und Journalismus“, „Wirtschaft und Administration“, „Symbole“, „Historische Fußballmomente“ und die „WM 2006“.

4. Der Bildung entsprechender Kategorien folgte die erste Makroanalyse von Metatexten $^{\text {iv }}$, indem die gefundenen ,kulturellen Deutungsmuster ${ }^{6}$ mit aktueller Sekundär- bzw. Forschungsliteratur verglichen wurden. An dieser Stelle ging es darum, den Hintergrund von Deutungsmustern, der sich üblicherweise nicht der intuitiven Einsicht der Mitglieder einer Kommunikationsgemeinschaft ${ }^{v}$ erschließt (Altmayer 2005: 157), zu systematisieren. Dieser Schritt erscheint deswegen sinnvoll, da möglicherweise auch das Forschersubjekt bestimmte Deutungsmuster erst versteht, wenn es mit Hilfe von Meta- bzw. Sekundärtexten weitere Erklärungen über selbige zu Rate gezogen hat.

5. Der ersten folgte eine zweite Makroanalyse, indem die bisher gefundenen ,deutschen kulturellen Deutungsmuster' mit Artikeln aus der argentinischen und brasilianischen Presse konfrontiert wurden. In diesem kontrastiven Vergleich ging es um die Frage, inwieweit verschiedene Kommunikationsgemeinschaften dem Phänomen „Fußball“ auf verschiedene Weise Sinn zuschreiben, ob sich also die Muster in den Diskursen voneinander unterscheiden (Altmayer 2006: 52). Hier erfolgte nach jeder Analyse eine kleine Zusammenfassung der Ergebnisse. 
6. Die eigentliche Interpretationsarbeit erfolgte in einem letzten Schritt im Zuge der Kontextualisierung. Dabei wurden einerseits Thesen aufgestellt, die den Umgang einer bestimmten Kommunikationsgemeinschaft mit dem „Fußballdiskurs“ betreffen, und andererseits bestimmte ,kulturelle Deutungsmuster ${ }^{6}$ und ihr Potenzial noch einmal thematisieren. Mithilfe dieser Thesen können die Ergebnisse der Analyse auf ein relevantes Anwendungsgebiet zurückgeführt werden (Altmayer 2007: 274): Die Beschreibung bestimmter Deutungsmuster, bspw. durch die Einbettung in einen bestimmten historischen oder sozialen Kontext, zeigt die Tiefenstruktur auf, die einem Diskurs zugrunde liegt. Auf diese Weise können Sprachlerner angeregt werden, die ihnen bisher zur Verfügung stehenden Deutungsmuster mit den „,neuen“ Mustern zu vergleichen und so zu einem umfassenderen Textbzw. Diskursverständnis gelangen.

Um deutlich zu machen, auf welchen Elementen die kulturwissenschaftliche Diskursanalyse beruht, sei an dieser Stelle zunächst noch einmal eine (stark komprimierte) Definition von ,kulturellen Deutungsmustern'vorangestellt. Für eine ausführliche Herleitung und Definition des Konzepts und des entsprechenden Analyseverfahrens sei auf Altmayer (u. a. 2002: 16) verwiesen.

Bei Fragen der kulturwissenschaftlichen Diskursanalyse geht es letztendlich darum nachzuvollziehen, wie Menschen als Individuen ihre Umgebung und Wirklichkeit deuten. Grundlegend ist diese Frage für die zwischenmenschliche Kommunikation: Kommunikationspartner müssen zunächst von einem ähnlich gearteten Vorwissen ausgehen können, um sich überhaupt verstehen zu können. Die dabei verwendeten Muster müssen nicht jedes mal wieder neu definiert werden, sondern bilden „eine Art offene[n] Fundus, aus dem sich die Individuen für die Deutung und Bewertung von Situationen, Texten usw. nach eigenem Gusto bedienen können“ (Altmayer 2006: 52f.), das heißt, sie stehen einer Gruppe als gemeinsamer Wissensvorrat für die gemeinsame diskursive Wirklichkeitsdeutung zur Verfügung. Sehr verknappt kann also folgende Definition zum Tragen kommen: Bei ,kulturellen Deutungsmustern' handelt es sich einerseits um Strategien, mit denen sich Individuen potenziell die sie umgebende Welt erklären, und andererseits um die Menge des ,kulturellen Wissens,, das Kommunikationsgemeinschaften als Grundlage für ihre Kommunikation zur Verfügung steht.

Hier schließt sich der Kreis zur Analyse: Um beschreiben zu können, wie innerhalb einer bestimmten Kommunikationsgemeinschaft kommuniziert wird, findet ein Verfahren Verwendung, das die in dieser Gruppe öffentlich zirkulierenden Texte untersucht. Diese Texte sind Repräsentationen der Diskurse, die innerhalb dieser Kommunikationsgemeinschaft zum Tragen kommen. Bei Verfahren der kulturwissenschaftlichen Diskursanalyse werden die Diskurse einer Kommunikationsgemeinschaft nun aus einer ganz bestimmten Perspektive heraus betrachtet: Es geht darum, einzelne ,kulturelle Deutungsmuster ${ }^{6}$ zu erkennen, offen zu legen und zu beschreiben. 


\section{Ein Beispiel: Die „Nummer 10“}

Die Diskussion über ,kulturelle Deutungsmuster' findet zugegebener Weise auf einer hohen Abstraktionsebene statt. Um besser zu veranschaulichen, wie das Konzept der ,kulturellen Deutungsmuster' für das Verfahren einer kulturwissenschaftlichen Diskursanalyse genutzt werden kann, sei an dieser Stelle aus vielen möglichen Kandidaten ein Deutungsmuster aus dem Bereich Fußball herausgegriffen: Das ,kulturelle Deutungsmuster „Nummer 10“. Einerseits ist die „10“ selbstverständlich eine ganz normale Zahl und ständig in unserem Alltag präsent, andererseits ist sie aber auch eine Zahl mit gewissem Symbolwertvi. Hier wird nun der Vergleich mit dem Fußballdiskurs interessant, denn dort ist die „10“ nicht nur eine einfache Nummer. Dies illustrieren die nun folgenden Zitate.

Im Streit um die Rolle des Führungsspielers der brasilianischen Nationalmannschaft musste Weltfußballer Ronaldinho Gaúcho im Frühjahr 2007 vorübergehend das Trikot mit der „Nummer 10“ abgeben. Die Süddeutsche Zeitung schrieb dazu:

[... ] Auf dem Rücken trug er die 7. Aber in ihm wird Ronaldinho immer die 10 sein. Er lächelte und sagte: „Es ist doch egal, was auf dem Rücken steht. Wenn wir für Brasilien spielen, sind wir alle dieselbe Nummer. Die Nummer eins." [ ... ] War es seine sanfte Ironie, oder seine Art des stillen Protests? Er tauschte in Schweden seinen Ohrring. Er trug einen gewöhnlichen Brillanten statt seines üblichen, funkelnden Ohrsteckers R10. vii

Auch um den Spielmacher der deutschen Nationalmannschaft entbrannte eine heiße Diskussion, was sich nicht zuletzt an Umfragen in einem Web-Blog viii beispielhaft aufzeigen lässt:

[ ... ] Derzeit will wohl keiner der deutschen Spieler so recht das Trikot mit der Nummer 10 und damit die Spielmacherposition übernehmen. Der einzigste wo [sic!] im deutschen Spiel dafür eigentlich in Frage käme, wäre Michael Ballack, welcher allerdings seine „13“ bevorzugt. Was meint ihr, wer soll das Trikot [mit der 10] tragen? [ ... ]

$\begin{array}{lc}\text { Michael Ballack } & 5 \% \\ \text { Sebastian Deisler } & 5 \% \\ \text { Lukas Podolski } & 23 \% \\ \text { Sebastian Schweinsteiger } & 5 \% \\ \text { niemand } & 11 \% \\ \text { ein anderer } & 47 \%\end{array}$

Was für die Nationalmannschaft gilt, lässt sich auch für den FC Bayern feststellen, der vor allem im Ausland wohl wie wenige andere Vereine als Vertreter des deutschen Fußballs wahrgenommen wird. In einer Kolumne der Bildzeitung findet sich folgender Kommentar: 
[ ... ] Die breit angelegte Diskussion um eine Nummer 10 für den FC Bayern, also um einen Spielmacher oder Regisseur, geht an der Aktualität vorbei. Weil der Fußball von heute sie längst überholt hat. Dieses Thema erinnert mich an die Zeit, als Franz Beckenbauer in der Nationalmannschaft aufgehört hatte. Franz, der beste Libero aller Zeiten. $[\ldots]^{\text {ix }}$

Spätestens nach dem dritten Verweis auf die „Nummer 10“ im Zusammenhang mit Fußball wird deutlich, dass die Symbolik der „10“ über die alltäglichen Zuschreibungen hinausgeht. Auf der Suche nach einer Erklärung dafür, was die „10“ im Zusammenhang mit dem Ballsport symbolisiert, lässt sich im eingangs zitierten Artikel der Süddeutschen Zeitung folgender Hinweis finden: „Die 10 steht für Eleganz, für Anmut, für Einzigartigkeit $[\ldots]^{] ‘}$.

Woher kommt nun die Zuschreibung der genannten Eigenschaften zu der Zahl? Über die historischen Hintergründe der Verbindung findet sich im Internet folgender Hinweis:

Im Jahr 1958 hörte die 10 auf, eine Nummer zu sein. Bei der FußballWeltmeisterschaft in Schweden stürmte ein 17-jähriger Junge für Brasilien, auf dem Rücken trug er die 10, und als er im Endspiel den Ball mit der Brust stoppte, über einen Gegner hinweglupfte und volley ins Tor schoss, hatte sich die Welt verändert. Die 10 hatte begonnen, ein Mythos zu werden, ein Gesandter übersinnlicher Magie auf Erden; das Erkennungs-zeichen des besten Fußballers im Team. Der 17-jährige Junge hieß Pelé $[\ldots] .{ }^{x}$

Etwas weniger emotional gibt sich die Online-Ausgabe des Spiegel:

Über die Jahre wurden die Nummern der Einfachheit halber den Spielpositionen zugeordnet. Die Nummer 5 gehört spätestens seit Franz Beckenbauer dem freien Mann vor der Abwehr, die 10 seit Ferenc Puskás den genialen Regisseuren, die 9 dem Mittelstürmer, die 7 dem Rechtsaußen. ${ }^{x i}$

Was haben wir bis hierher über die „Nummer 10“ erfahren? Um wieder zur MetaEbene zurückzukehren, müssen wir an dieser Stelle den oben erwähnten Schritt der Kontextualisierung als Teil der Analyse vollziehen. Wenn wir die Aussagen aus der Süddeutschen Zeitung, der Bild und dem Spiegel zusammenfassen, handelt es sich bei der „Nummer 10“ um einen Fußballspieler, der die Nummer 10 auf seinem Trikot trägt und im Mannschaftsgefüge die Position des Spielmachers oder „Regisseurs“ inne hat. Die 10 ist ein „Mythos“, eine „magische Nummer“ für „Superstars“, die an „geniale Feldspieler" vergeben wird. Diese Nummerierung ist seit den 1950ern gebräuchlich und wird momentan teilweise von Fußball-Analysten hinterfragt. Assoziiert wird die Nummer mit Namen wie Pelé, Maradona, Zidane, Ronaldinho, aber auch Lothar Matthäus und Oliver Neuvillexii.

Das Bild der „Nummer 10“ taucht in verschiedensten öffentlich zirkulierenden Texten auf, die zum Teil gegenseitig aufeinander Bezug nehmen. Wir können also davon ausgehen, dass es sich hierbei, zumindest was den Fußballdiskurs angeht, tatsächlich um ein Deutungsmuster handelt: Die „Nummer 10“ erweckt positive Assoziationen, sei es hinsichtlich der Ästhetik des Spiels (Eleganz, Anmut), der 
Besonderheit (Einzigartigkeit, Genialität, Mythos, übersinnliche Magie), der Führungsstärke (Regisseur, Spielmacher, bester Spieler) oder einfach nur im Verweis auf herausragende Spielerpersönlichkeiten (die Ausnahmespieler Pelé, Maradona oder Zinedine Zidane), die im kollektiven Gedächtnis einer Kommunikationsgemeinschaft einen zentralen Platz einnehmen können. Dass nicht alle Mitglieder der deutschen Kommunikationsgemeinschaft über die Gesamtsumme der hier dargestellten Deutungsmuster verfügen ist selbstverständlich. Allerdings steht es außer Frage, dass es sich um ,kulturelle Deutungsmuster ${ }^{6}$ handelt, die potenziell für den deutschsprachigen Diskurs über Fußball abgerufen werden können.

Spätestens an diesem Punkt stellt sich eine weitere Frage: Ist das ,kollektive Wissen“, das hinter der „Nummer 10“ steht, auf die deutsche Kommunikationsgemeinschaft, ihr Denken und ihren Diskurs beschränkt, oder lässt sich eine ähnliche Verwendung auch für andere Kommunikationsgemeinschaften bzw. in anderen Sprachen nachweisen? Diese Frage motivierte in meiner Magisterarbeit die zweite Makroanalyse, den kontrastiven Vergleich von deutschen Pressetexten mit OnlineArtikeln brasilianischer und argentinischer Zeitungen. Ohne an dieser Stelle ins Detail gehen zu wollen: Auch für Argentinien (Paradebeispiel: „El Diego“ Maradona) und Brasilien (Pelé als ewiger Torschützenkönig) spielt die „Nummer 10“ eine wichtige Rolle - offensichtlich mehr noch als für Deutschland. Dass es sich nur wenige brasilianische Zeitungen versagen, sich in Kommentaren über Fußball auf die Ausnahmefigur des nationalen Ballsports, Pelé, zu beziehen, sei durch folgendes Zitat aus einem Artikel illustriert, der am 02.07.2006 (unmittelbar nach dem Ausscheiden der Seleção) in der Folba de São Paulo erschien und die vergangenen Spiele Ronaldinho Gaúchos in der brasilianischen Nationalmannschaft kritisch hinterfragt:

O jogador do Barcelona completou o aniversário de seca durante a competição e definitivamente não honrou a camisa 10 que serve até de campanha publicitária da Nike -o número que já foi usado por monstros sagrados do futebol brasileiro nos Mundiais, como Pelé, Rivellino e Zico.

Der Spieler des FC Barcelona konnte auch während der Weltmeisterschaft seine ein Jabr andauernde Durststrecke nicht beenden und ehrte damit definitiv nicht das Trikot Nummer 10, das sogar den Werbespot von „Nike” prägt - die Nummer, die bereits die „Heiligen Übermenschen" des brasilianischen Fußballs bei Weltmeisterschaften trugen, wie etwa Pelé, Rivellino und Zico. [Freie Übersetzung, P.V.]

Es bietet sich an, zum Verständnis dieses Textabschnittes über das episodische Wissen zu verfügen, dass Ronaldinho Gaúcho 2005/2006 zwar beim FC Barcelona die beste Clubsaison seines Lebens gespielt hatte, aber für die Nationalmannschaft in über einem Jahr kein Tor erzielen konnte. Aufschlussreicher für unsere Fragestellung erscheint allerdings, dass auch im brasilianischen Fußballdiskurs mit ähnlichen Deutungsmustern gearbeitet wird wie im deutschsprachigen.

Deutlich wird die Bedeutung der „10“ in Südamerikaxiii an zwei weiteren Beispielen, die den Rahmen einer kulturellen Diskursanalyse im engeren Sinne bereits 
verlassen. Das erste: Bei einer im Oktober 2007 in Belém (Nordbrasilien) durchgeführten Erhebung konnte ich feststellen, dass von 191 im Straßenbild gezählten Personen, die eindeutig eine Zahl auf ihrem Fußball-Trikot trugen, 120 Personen (oder 63\%) mit einem Trikot mit der „Nummer 10“ bekleidet waren. Die Zahlen unterstreichen den subjektiven Eindruck, dass fast zwei Drittel der Fußballtrikots, die einem im brasilianischen Alltag begegnen, mit dieser Nummer versehen sind ${ }^{\text {xiv }}$.

Das zweite Beispiel betrifft den Sprachvergleich: Sowohl im Spanischen Argentiniens als auch im Portugiesischen Brasiliens ist die Zahl „10“ Ausdruck dafür, dass alles perfekt ist - in beiden Ländern kann man auf die Frage nach dem Wohlergehen („¿Cómo andás?“ bzw. „Como vai?“) mit der Redewendung „De 10!“ bzw. ,Tudo 10!“ antworten, wenn man deutlich machen möchte, dass alles in bester Ordnung ist. Auch Produkte oder Dienstleistungen können im Zweifelsfalle mit „de 10“ betitelt werden, wenn sie vor den Augen des Sprechers ohne Tadel bestehen. In weiten Teilen Argentiniens und Brasiliens ist die „10“ zudem die beste Note in Schule oder Universität und entspricht der deutschen „1“. Eine Parallele also zu den oben erwähnten besonderen Assoziationen zur Zahl 10 im deutschsprachigen Diskurs, und gleichzeitig eine Erweiterung hin zu einer deutlich positiven Einschätzung.

Es könnte argumentiert werden, dass mit dem letzten Absatz der Rahmen der vorher erwähnten Kontextualisierung bereits überschritten sei. Dieses Argument relativiert sich allerdings, wenn man sich ins Gedächtnis ruft, dass mit Hilfe der ,kulturellen Deutungsmuster ${ }^{6}$ ein Versuch unternommen wird, die individuelle Konstruktion von Wirklichkeit auf der Basis kollektiv geteilten Wissens verstehend nachzuvollziehen. Wenn sich also thematische Überschneidungen bei der Verwendung eines bestimmten Deutungsmusters nachweisen lassen, ist nicht auszuschlieBen, dass die Thematisierung desselben, die Bewusstmachung der Deutungen also, generell beim Verstehensprozess helfen kann. Gleichzeitig unterstreicht das Verfahren der kulturellen Diskursanalyse noch einmal deutlich, dass für das Verstehen eines Diskurses weit mehr vonnöten ist als reine Vokabelkenntnis - und dass es auf alle Fälle sinnvoll sein kann, einem transdisziplinären Ansatz unter Rückgriff auf andere Fächer zu folgen.

An dieser Stelle kann wiederum der Kreis zur eingangs geäußerten Überzeugung geschlossen werden, dass Fremdsprachen- und kulturelles Lernen untrennbar miteinander verbunden sind. Um es noch einmal deutlicher zu formulieren: Es ist unabdingbar, dass Fremdsprachenlerner nicht nur Wörter und Sätze lernen, sondern auch mit den möglichen (selbst widersprüchlichen) Deutungen von Wirklichkeit konfrontiert werden, die in einem (in unserem Falle fremdsprachlichen) Diskurs nebeneinander stehen. Nur so ist es ihnen möglich, erfolgreich am Diskurs der Zielsprache zu partizipieren (vgl. Altmayer 2006: 54) und letztendlich erfolgreich mit Zielsprache und -kultur umgehen zu können. 


\section{Mögliche Probleme einer kulturwissenschaftlichen Diskursanalyse}

Bei allem Enthusiasmus für die Möglichkeiten einer kulturwissenschaftlichen Diskursanalyse sollte jedoch nicht übersehen werden, dass eine Reihe von Fragen im Bezug auf das Verfahren noch nicht zufriedenstellend geklärt werden konnten. Insbesondere betrifft das die vier Problemfelder, die in der Einführung bereits erwähnt wurden und nun zunächst besprochen werden sollen.

\section{Problem 1: Kodierung}

Der Hintergrund dieses Problems ist die Tatsache, dass die Texte, die die Grundlage einer Analyse bilden sollen, in ihrer Gesamtheit vorliegen und zunächst geordnet werden müssen, damit eine systematische Betrachtung überhaupt erst möglich wird. Die Frage ist nun, nach welchen Gesichtspunkten ein Ordnen der Texte sinnvollerweise erfolgen sollte. Nach den Erfahrungen meiner Arbeit erfolgt diese Anordnung am günstigsten anhand von Kategorien. Diese Kategorien bilden die „Kodes“, mit denen gearbeitet wird, um zu den hinter den Texten liegenden ,kulturellen Deutungsmustern' vorzudringen. Die Kodierung - oder Kategorienbildung - stellt demnach die erste Phase in der Sequenz der Analyse dar.

Meine Frage in dieser Phase zielte zunächst auf die Gesamtheit der Pressetexte: Worüber wird im jeweiligen Artikel geschrieben? Es ging also darum, alle Texte nach inhaltlichen Gesichtspunkten zu lesen und aufmerksam zu verfolgen, welche Themen sich in ihnen wiederholten. Nach dem Sichten der Texte wurden zwei Kategorien deutlich, die sich wiederum in Unterkategorien aufteilen ließen, nämlich:

a) „Fußball als Spiel“, das heißt im unmittelbaren Spielgeschehen („deutsche Fußball-Nationalmannschaft", „Deutsche“, „Fußballfans“, „beteiligte Personen“), und

b) das „Umfeld des Fußballs“ („Presse und Journalismus“, „Politik““, „Wirtschaft und Administration“, „Symbole“; dazu in einem zweiten Schritt noch „Historische Momente" und die „WM 2006").

Mithilfe dieses Vorgehens wurden die Kodes deutlich, an denen ich mich im Rahmen der Feinanalyse der Basistexte (Schritt 2) orientieren wollte.

Offen bleibt hier die Frage, ob Kategorien günstiger anhand eines deduktiven oder induktiven Vorgehens etabliert werden sollten - trägt man also bereits im Vorfeld aufgestellte Thesen an die Texte heran oder entwickelt man die Kategorien besser aus dem Material heraus? Diese Frage muss jedes Mal von Neuem unter Berücksichtigung des Gegenstandes und des gewählten Diskurses beantwortet werden (vgl. Altmayer 2007: 275). Darüber hinaus bleibt die Frage, ob sich möglicherweise objektivere Kriterien für eine Strukturierung von Texten finden lassen. 


\section{Problem 2: ,Kulturelle Deutungsmuster'-Eingrenzung und Gebrauch}

Nach der Frage nach dem Inhalt (wor̈̈ber wird geschrieben?) stellt sich im Umgang mit ,kulturellen Deutungsmustern' sodann die Frage nach dem wie - es geht im Wesentlichen also um die unterschiedlichen Perspektiven, unter denen ein Text verstanden und interpretiert werden kann. Dabei muss die Grenze zu ,Alltagswissen' scharf gezogen werden, damit nicht fälschlich aus episodischem oder individuellem Wissen ein allgemein gültiges Deutungsmuster konstituiert wird. Bei Überschreitung dieser Grenze ist die Gefahr groß, dass das Konzept der ,kulturellen Deutungsmuster ${ }^{6}$ unscharf wird und in eine Beliebigkeit abgleitet, die kein sinnvolles Arbeiten mehr zulässt. ,Kulturelle Deutungsmuster' gehen per definitio tiefer als Alltagswissen und erschließen sich dem Leser nicht unbedingt auf den ersten Blick - es geht also darum, wirkliches Hintergrundwissen zu erkennen, das einem Text zugrunde liegt, wenn nach einem objektiv nachvollziehbaren Verstehen gefragt wird. Weitere offene Fragen liegen speziell bei der Eingrenzung (Wo höre ich auf, nach weiteren Mustern eines Diskurses zu suchen?) und dem Gebrauch (Was mache ich für eine schlüssige Kontextualisierung?) von ,Kulturellen Deutungsmustern'.

\section{Problem 3: Tiefenstruktur von Diskursen}

Nicht erst durch Verfahren der kulturwissenschaftlichen Diskursanalyse wird deutlich, dass es sich bei Diskursen um unglaublich kompliziert und bunt aufgebaute Konstruktionen handelt - ebenso vielseitig wie das Leben selbst, da durch ebenso viele Individuen gestaltet, wie an ihnen teilnehmen. Dabei handelt es sich bei Diskursen keineswegs um statische Gebilde, sondern um sich fortwährend verändernde Manifestationen menschlichen Denkens.

Eine Möglichkeit sich Diskursen zu nähern liegt in der von Altmayer immer wieder genutzten Metapher des ,Hypertexts' (z. B. Altmayer 2007: 269), nach deren Idee die Deutung eines Textes immer wieder auf andere Texte verweist. Aber auch hier muss klar sein, dass unmöglich ein gesamter ,Diskurs‘ analysiert werden kann, sondern stets nur ein (kleiner) Ausschnitt. Vielleicht muss aber auch ein Diskurs nicht in seiner ganzen Breite beschrieben werden, wenn es vornehmlich um ein angemessenes (und nicht komplettes) Verstehen von Texten geht?

Der von mir beschrittene Weg auf der Suche nach der Tiefenstruktur von Diskursen besteht darin, mithilfe der Beschreibung ,kultureller Deutungsmuster möglichst umfassend einen Diskurs nachzuvollziehen. Doch auch hier bleiben sicher noch Fragen dazu offen, bis zu welcher Tiefe ein solcher durch ein so deutlich textbezogenes Verfahren tatsächlich erfasst und beschrieben werden kann - oder für das Verständnis eines Textes überhaupt erfasst und beschrieben werden muss. 


\section{Problem 4: Konkrete Anwendung von Ergebnissen einer Diskursanalyse}

Die sicher spannendste Frage stellt sich nach der Verwendung von Ergebnissen einer kulturwissenschaftlichen Diskursanalyse, denn durch das Aufzeigen der Struktur eines Diskurses allein finden Lerner nicht automatisch auf den Weg des „kulturellen Lernens“. Wichtig sind dabei ohne Zweifel drei Dinge:

a) Erstens sollte die Forschungsarbeit mit Verfahren wie der in diesem Artikel beschriebenen Diskursanalyse intensiviert werden. Denn über eine größere Anzahl von Kulturanalysen kann die Kulturwissenschaft $\mathrm{DaF}$ sich ihrem Ziel nähern, „durch die Sichtbarmachung kultureller Deutungsmuster Deutsch als Fremdsprache-Lernern ein Potenzial an kulturellen Bezügen bereit zu stellen, das diese für ihren individuellen Verstehens- und Sinnbildungsprozess nutzen und mit ihrem eigenen kulturellen Potenzial in eine sinnvolle Verbindung bringen können" (Altmayer 2006: 57) und damit ,kulturelles Lernen' anregen.

b) Neben weiteren Kulturanalysen erscheint es unabdingbar, auch die empirische Forschung voranzubringen (ebd.). Bis jetzt liegen kaum entsprechende Arbeiten vor - auch in der vorgestellten Magisterarbeit erfolgte zwar eine Auseinandersetzung mit Methoden und Instrumenten kulturwissenschaftlicher Fragestellungen, die aufgestellten Thesen konnten aber nicht mit empirischen Untersuchungen an Probanden oder Gruppen belegt werden. Hier gibt es sicher ein großes Potenzial für wissenschaftliche(s) Arbeiten.

c) Schließlich sollte nicht vergessen werden, die Ergebnisse der Diskursanalyse in den Fremdsprachenunterricht zurückzutragen. Mit der Idee der ,inszenierten Teilhabe an zielsprachlichen Diskursen" (Altmayer 2006: 54, 56) lassen sich bereits handfeste Vorschläge für das Fördern von Lernprozessen finden. Fast noch wichtiger erscheinen mir die Ergebnisse für eine angemessene Themenplanung eines DaF-Unterrichts, die dank einer Kulturwissenschaft DaF eine wissenschaftliche Grundlage bekommen kann. Hierzu werden im abschließenden Ausblick noch einige kurze Überlegungen angestellt.

\section{Ergebnisse der Arbeit}

Das hier vorgestellte Vorgehen der kulturwissenschaftlichen Diskursanalyse fand nicht nur bei der Beschreibung der „Nummer 10“ Verwendung, sondern bei einer Reihe weiterer Konzepte, die dem Fußballdiskurs zugrunde liegen und ebenso unter ,kulturellen Deutungsmustern' subsumiert werden können. Mit der Zusammenführung, dem Vergleich und der Interpretation von Deutungsmustern aus den Diskursen drei verschiedener Kommunikationsgemeinschaften wurden darüber hinaus mehrere Beobachtungen deutlich, die im Folgenden stark komprimiert als Thesen dargestellt werden. 


\section{These 1:}

Im deutschsprachigen Diskurs wird dem Umfeld des Fußballs eine größere Bedeutung beigemessen als im spanisch- bzw. portugiesischsprachigen. Auf der anderen Seite wird in Südamerika Fußball entschiedener als genuiner Teil der (Alltags-) Kultur gesehen (Gebauer 2000: 155). Laut Aussagen Schröders (2000: 63) muss diese Präsenz des Fußballs in der ,lateinamerikanischen Kultur“ nicht extra thematisiert oder ausgehandelt werden, sondern wird als Tatsache angenommen. Aus diesem Grund geht es im Fußballdiskurs Argentiniens und Brasiliens auch mehr um das Spiel (und seine Qualität) selbst als um das Umfeld (Martínez 2000: 11).

\section{These 2:}

Durch die stärkere Einbindung in die ,Kultur ${ }^{\varsigma}$ werden im südamerikanischen Diskurs jeweils auch ganz bestimmte Erwartungen geschürt. So gibt es ein genau definiertes Bild des ,jogo bonito“ in Brasilien und eines eigenständigen „La-PlataStils“ (Archetti 1997: 15; 1995: 205, 211) in Argentinien. Letztendlich ist ein ,nationaler Stil"، im Fußball jedoch spieltechnisch nicht nachweisbar, sondern ein gern gepflegter Mythos (Archetti 195: 217; Gebauer 2000: 162). Interessant hingegen sind die Vorstellungen, die aus dem Alltagsleben auf den Fußball projiziert werden. Der zunehmende Gebrauch von Fußballmetaphern im Alltag ist nur ein Beispiel hierfür.

\section{These 3:}

Über die Grenzen von ,isolierten“xv Kommunikationsgemeinschaften hinweg funktioniert der Diskurs über Fußball ähnlich, weil selbst über das eigentliche Spielgeschehen hinaus viele Übereinstimmungen in den evozierten ,kulturellen Deutungsmustern' auftreten. Diese Ähnlichkeiten in der Interpretation von Fußball beschränken sich eben keinesfalls nur auf die „Nummer 10“, sondern erstrecken sich bspw. auch auf, kulturelle Deutungsmuster" wie die „Hand Gottes“, Schlüsselereignisse (wie etwa die WM 1954 für Deutschland, die WM 1978 für Argentinien oder das Finale der WM 1950 für Brasilien) ${ }^{\text {xvi }}$ oder herausragende Spieler (wie etwa Gerd Müller, Franz Beckenbauer und Lothar Matthäus oder Diego Maradona und Pelé)xvii. Auch über konkrete Ereignisse oder Personen hinaus lassen sich ähnliche Deutungsmuster ausmachen: Fußball wird in jedem Falle als ,kollektive Erfahrung' gesehen, die sich in einer bestimmten Form (auch) auf das Gemeinschaftsgefühl auswirkt. Fußball ist zudem ein Phänomen, das eine große Begeisterung auslösen und überall eine unerklärliche Faszination ausüben kann. (Pornschlegel 2002: 105; Martínez 2002: 7; Hütig \& Marx 2004: 12, 17).

Während die ersten beiden Thesen vor allem (auch im Unterrichtsalltag) zu einer kritischen Betrachtung über den Umgang mit Texten zum Thema Fußball einladenxviii, hat die dritte These möglicherweise sehr viel weiter reichende Konsequenzen. Diese These impliziert nämlich den Gedankengang, dass ein Individuum 
nicht nur Teil einer einzigen abgeschlossen Kommunikationsgemeinschaft sein kann, die jeweils mit Hilfe potenziell gleicher ,kultureller Deutungsmuster ${ }^{6}$ kommuniziert, sondern an mehreren Kommunikationsgemeinschaften mit einem jeweils spezifischen Diskurs teilhaben kann. So ist ein Deutschlerner möglicherweise von Haus aus Teil der brasilianischen Kommunikationsgemeinschaft, hat aber Englisch gelernt und kann dadurch an englischsprachigen Diskursen teilnehmen. Darüber hinaus könnten Kommunikationsgemeinschaften auch jenseits von Sprachgrenzen gesucht werden: Die Mitglieder eines Ruderclubs oder eines Ornithologenvereins nutzen im Zweifelsfalle sehr spezifische Diskurse, für die sie auf ganz eigene Deutungsmuster zurückgreifen müssen und die für einen Großteil der entsprechenden sprachlichen Kommunikationsgemeinschaft unverständlich sind.

Als Konsequenz daraus lässt sich eine weitere Überlegung erhärten: Es gibt bestimmte Themen der menschlichen Kommunikation, die als universal gelten können - wenn man so will, ist auch Fußball eines davon. Ob man von ,universellen Daseinserfahrungen' im Sinne von Neuner (1989) spricht oder aktuellere Modelle zu Rate zieht (siehe bspw. das Vier-Kategorien-Modell in Altmayer 2006: 56), ist an dieser Stelle weniger von Belang: Wichtig erscheint die Aussage, dass bestimmte Themen für den Sprachunterricht deutlich effektiver nutzbar wären als dies gegenwärtig der Fall ist. Nicht nur durch die modernen Medien ist die Welt von heute so sehr miteinander verflochten, dass einem Lerner nicht mehr nur ,kulturelle Deutungsmuster' der eigenen (bspw. deutschen) Kommunikationsgemeinschaft zur Verfügung stehen, sondern diese zunehmend universal erscheinen. Was dies für die fremdsprachliche Unterrichtspraxis für Konsequenzen haben kann, wird im abschließenden Ausblick noch einmal zusammenfassend diskutiert.

\section{Zusammenfassung und Ausblick}

Nachdem bis hierher aufgezeigt wurde, auf welcher theoretischen Basis die Magisterarbeit beruhte, die eingesetzten Verfahren beschrieben und die Ergebnisse benannt wurden, soll es abschließend um mögliche Konsequenzen unserer Untersuchung gehen.

Ein erstes Ergebnis liegt in der Beschreibung eines Diskurses, indem exemplarisch der Fußballdiskurs in mehreren Kommunikationsgemeinschaften analysiert wurde. Dadurch konnte deutlich gemacht werden, dass Texte als Repräsentanten des menschlichen Denkens, vor allem aber Diskurse sehr breit gefächert sein können, sich sowohl auf sich selbst als auch auf die Gesellschaft beziehen und heterogen bis widersprüchlich sein können. Einmal mehr erhärtet sich also die These, dass für das Verstehen von Texten durchaus mehr notwendig ist als reine Vokabelkenntnis: Es bedarf auch einer umfassenden Kenntnis des Kontexts und eines bestimmten Vorwissens, um den Sinn eines Textes schlüssig (re-)konstruieren zu können. Mit Hilfe von ,kulturellen Deutungsmustern' steht nun ein Konzept zur Verfügung, um die große Bandbreite an Aspekten, die einem Diskurs 
zugrunde liegen, sozusagen zu kanalisieren und einen Diskurs damit überhaupt erfassbar zu machen. Dies ist wiederum eine Grundvoraussetzung dafür, fundierte Antworten auf die Fragen zu geben, die aus der DaF-Praxis in die WissenschaftsPraxis getragen werden (Altmayer 2006: 56).

Bei der Konstruktion von Wirklichkeit (Altmayer 2006: 51) liegt das zweite Ergebnis, indem aus der Perspektive einer Kulturwissenschaft DaF nachvollzogen wird, wie der Verstehensprozess eines Diskurses funktionieren kann. Mithilfe einer umfassenden Textanalyse wurde gezeigt, welche ,kulturellen Deutungsmuster ${ }^{6}$ dem Diskurs über Fußball in einer bestimmten Kommunikationsgemeinschaft potenziell zugrunde liegen. Durch die explizite Darstellung der möglichen Deutungsmuster wurde eine Strategie aufgezeigt, anhand derer durch den bewussten Vergleich verschiedener Texte eines gleichen Diskurses die Kompetenz von Fremdsprachenlernern geschult werden kann, sich auf die Heterogenität von Diskursen und auf mögliche weitere Lesarten von Texten einzustellen.

Wenn dem Phänomen der ,kulturellen Deutungsmuster ${ }^{6}$ die nötige Aufmerksamkeit entgegengebracht wird, dann ist dies eine Möglichkeit für die Lerner, weitere Diskurse zunächst zu verstehen und (aktiv) an ihnen teilzunehmen. Hier ist der Bogen geschlagen zu der eingangs aufgestellten Forderung, über die Partizipation an zielsprachlichen Diskursen in Prozesse des ,kulturellen Lernens' einzutreten (Altmayer 2006: $53 \mathrm{f}$ ).

Als drittes - und meiner Meinung nach für die Unterrichtspraxis zunächst relevantestes - Ergebnis sei nochmals auf die dritte der im sechsten Kapitel genannten Thesen verwiesen: Hier war deutlich geworden, dass in der zwischenmenschlichen Kommunikation bestimmte Themen auftreten, die unabhängig der Zugehörigkeit des Individuums zu einer spezifischen Kommunikationsgemeinschaft als universal angesehen werden können. Lerner einer Fremdsprache, egal welche Muttersprache sie sprechen, verfügen also über einen bestimmten Fundus ähnlicher ,kultureller Deutungsmuster'. Wenn diese Deutungsmuster auch, kulturspezifische‘ Variationen aufweisen mögen, die es zu beachten gilt, so verfügen die Lerner doch auf alle Fälle über ein großes Vorwissen - und dieses gilt es effektiv zu nutzen!

Es soll nun an dieser Stelle keineswegs die Forderung erhoben werden, das Thema Fußball als obligatorischen Bestandteil in den Fremdsprachenunterricht aufzunehmen. Eine Aufforderung bleibt allerdings bestehen: Auch wenn es für die Kulturwissenschaft DaF eine Fülle an Arbeit gibt, um ,kulturelles Lernen' auf eine solide wissenschaftliche Basis zu stellen, so kann durch eine bewusste Auswahl an Themen im Sprachunterricht, die das Potenzial sich ergänzender Deutungsmuster nutzt, heute schon eine deutliche Professionalisierung des Deutsch- und insbesondere Landeskundeunterrichts erreicht werden. 


\section{Literatur}

Adelmann, Ralf; Parr, Rolf \& Schwarz, Thomas (Hrsg.) (2003): Querpässe. Beiträge zur Literatur-, Kultur- und Mediengeschichte des Fußballs. Heidelberg: Synchron Wissenschaftsverlag.

Altmayer, Claus (2002): ,Kulturelle Deutungsmuster ${ }^{\varsigma}$ in Texten. Prinzipien und Verfahren einer kulturwissenschaftlichen Textanalyse in Deutsch als Fremdsprache. In: Zeitschrift für interkulturellen Fremdsprachenunterricht 6(3) [http:/ / zif.spz.tu-darmstadt.de; 20.09.2008].

Altmayer, Claus (2003): Deutsch als Fremdsprache und Kulturwissenschaft. In: Altmayer, Claus \& Foster, Roland (Hrsg.) (2003): Deutsch als Fremdsprache: Wissenschaftsanspruch Teilbereiche - Bezugsdisziplinen. Frankfurt/Main: Peter Lang, 109-134.

Altmayer, Claus (2005): Kulturwissenschaftliche Forschung in Deutsch als Fremdsprache. Acht Thesen zu ihrer Konzeption und zukünftigen Entwicklung. In: Deutsch als Fremdsprache 3/2005, 154-159.

Altmayer, Claus (2006): ,Kulturelle Deutungsmuster ${ }^{\star}$ als Lerngegenstand. Zur kulturwissenschaftlichen Transformation der ,Landeskunde‘. In: Fremdsprachen Lehren und Lernen 35, 44-59.

Altmayer, Claus (2007): Kulturwissenschaftliche Diskursanalyse im Kontext des Faches Deutsch als Fremdsprache - Ziele und Verfahren. In: Redder, Angelika (Hrsg.) (2007): Diskurse und Texte. Festschrift für Konrad Ehlich zum 65. Geburtstag. Tübingen: Stauffenberg Verlag, 267-276.

Bauer, Joachim (2005): Warum ich füble, was du fühlst. Intuitive Kommunikation und das Geheimnis der Spiegelneurone. Hamburg: Hoffmann und Campe.

Brussig, Thomas (2003): „Sich die ganze Welt vom Fußball her erklären“. Thomas Brussig im Gespräch mit Stefan Hermanns und Markus Hesselmann. In: Adelmann, Parr \& Schwarz (Hrsg.), 171-176.

Gebauer, Gunter (2000): Fußball: Nationale Repräsentation durch Körper-Inszenierungen. In: Fischer-Lichte, Erika \& Fleig, Anne (Hrsg.): Körper-Inszenierungen. Präsenz und kultureller $W$ andel. Tübingen: Attempto, 149-164.

Horne, John \& Manzenreiter, Wolfram (2004): Football goes East. Culture and the people's game in Asia. London [u.a.]: Routledge.

Hütig, Andreas \& Marx, Johannes (Hrsg.) (2004): Abseits denken. Fußball in Kultur, Philosophie und Wissenschaft. Kassel: Agon Sportverlag.

Keller, Reiner (2003): Kultur als Diskursfeld. In: Geideck, Susan \& Liebert, Wolf-Andreas: Sinnformeln. Linguistische und soziologische Analysen von Leitbildern, Metaphern und anderen kollektiven Orientierungsmustern. Berlin: De Gruyter, 283-305.

Martínez, Matías (Hrsg.) (2002): Warum Fußball? Kulturwissenschaftliche Beschreibungen eines Sports. Bielefeld: Aisthesis Verlag. 
Pornschlegel, Clemens (2002): Wie kommt die Nation in den Fußball? Bemerkungen zur identifikatorischen Funktion des Fußballs. In: Martínez (Hrsg.), 103-112.

Schiffer, Jürgen (2004): Fußball als Kulturgut. Geschichtliche, soziologische, ökonomische, rechtliche, politische und philosophische Aspekte. Eine kommentierte Bibliografie. Köln: Sport und Buch Strauß Verlag.

Schröder, Ulfert (2000): Brasilien. In: Huba, Karl-Heinz (Hrsg.) (2000): FußballWeltgeschichte. 1846 bis heute. Bilder, Daten, Fakten. München: Copress, 59-66.

Silva, Francisco Carlos Teixeira da (2006): Sobre as origens do Futebol na Alemanha. In: Hollensteiner, Stephan \& Kaufmann, Göz (2006): Pequeno Dicionário de Futebol Alemão. Publikation des DAAD-Regionalbüros, Rio de Janeiro, Bonn, 3-9.

Wagner, Christoph (2004): Wir sind wieder wer! In: Hütig \& Marx (2004), 145-160.

Witt, Harald (2001): Forschungsstrategien bei quantitativer und qualitativer Sozialforschung. In: Forum qualitative Sozialforschung 2(1) [http://www.qualitativeresearch.net; 20.09.2008].

i Fußball ist nur ein Thema unter vielen, das in Lehrmaterialien (zu unrecht) ein Schattendasein führt, obwohl es sich um ein Phänomen von weltumspannender Popularität handelt (Horne \& Manzenreiter 2004: 3).

ii Als Begründung dafür, dass Pressetexte das Korpus meiner Arbeit darstellen, soll an dieser Stelle der Hinweis genügen, dass die Pressetexte in Tageszeitungen grundsätzlich darauf abzielen, von möglichst vielen Personen verstanden zu werden und sich deswegen par excellence derjenigen 'kulturellen Deutungsmuster' bedienen, die als Allgemeingut gelten. Man darf also davon ausgehen, dass der verwendete Diskurs von der entsprechenden Kommunikationsgemeinschaft zumindest verstanden wird, wenn er nicht sogar im aktiven Sprachgebrauch Verwendung findet

iii Es handelt sich hier um vier Artikel aus der „Süddeutschen Zeitung“, die jeweils unmittelbar nach Spielen der deutschen Mannschaft veröffentlich wurden (am 20. 06., 25. 06. und [zweimal] 07. 07. 2006) und als prototypisch für den untersuchten Diskurs angesehen werden. Zum Begriff Basistext siehe auch den Beitrag von Isabell Maringer in diesem Band.

iv Als Metatexte werden hier die Texte bezeichnet, die nicht Teil des Korpus bilden, aber auf den Diskurs Bezug nehmen bzw. ihn aus einer jeweils spezifischen Perspektive näher erklären.

v Eine Kommunikationsgemeinschaft ist eine Gruppe menschlicher Individuen, die einen gemeinsamen Vorrat an Deutungsmustern teilen. Mithilfe dieses Konzepts kann vermieden werden, pauschalisierend bspw. von „den Deutschen“ zu sprechen - ein Ausländer, der an einem deutschsprachigen Diskurs teilnehmen kann, ist selbstverständlich Teil der ,deutschen Kommunikationsgemeinschaft“. Für eine ausführliche Definition sei auf Altmayer (2004: 149; 2006: 52) verwiesen.

vi Man denke etwa nur an die „10 Gebote“ in der Bibel (2. Buch Mose, 20) oder das Lied „10 kleine Jägermeister“ der Musikgruppe „Die Toten Hosen“. Die Bedeutung der Zahl Zehn als Grundlage unseres Dezimalsystems ist wohl auf die Anzahl der Finger an beiden Händen zurückzuführen. Schon der Antike galt die Zehn als vollkommene Zahl, weil sie alle Zahlen von 1 bis 9 in sich einschließt.

vii Abgerufen unter: http://www.sueddeutsche.de/sport/weltfußball/artikel/769/ 107662/. Zugriff im März 2007, heute zu finden über das Archiv der Süddeutschen Zeitung. 
viii Abrufbar unter: http://www.fussball-foren.net/fussball187.html. Artikel vom August 2005, Zugriff im März 2007 und am 20.09.2008.

ix Abrufbar unter: http://www.bild.t-online.de/BTO/sport/bundesliga/2007/04/29/breitneranstoss/trikot-10-bayern.html, Zugriff im April 2007 und am 20.09.2008.

x Abrufbar unter: http://www.sueddeutsche.de/sport/weltfussball/artikel/769/ 107662/. Zugriff am 28.03.2007 und am 20.09.2008.

xi Ursprünglich verwendeter Link: http://www.spiegel.de/sport/fusball/0,1518,447961, 00.html. Aktuell abrufbar über das Spiegel-Archiv unter dem anschaulichen Titel „Halb Mensch, halb Tier die Nummer vier" (Artikel vom 15.11.2006).

xii Diese Namen wiederum finden sich in den Zeitungsartikeln, die parallel zur WM 2006 erschienen sind.

xiii Die Formulierung „südamerikanischer Fußballdiskurs“ steht hier vor allem aufgrund des Stils, um nicht immer von „brasilianischer bzw. argentinischer Kommunikationsgemeinschaft“ sprechen zu müssen. Diese Verallgemeinerung sei insofern verziehen, als ich bei ausgedehnten Reisen durch Südamerika in anderen Ländern keine deutlichen Unterschiede zu den hier präsentierten Thesen antraf.

xiv Die hier erwähnte Beobachtung bietet schier unerschöpfliches Material für weitere Fragestellungen, die allerdings aus dem Rahmen einer „Kulturwissenschaft $\mathrm{DaF}$ “ herausfallen würden. Beispielsweise könnte gefragt werden, ob in Deutschland mehr als 1,5\% der Träger von Fußballtrikots Frauen sind (Wert der Umfrage in Brasilien), welche Bedeutung eine lokale Mannschaft in der Trikot-Hierarchie einnimmt (20\% der Trikots) und inwieweit ein Einzelspieler bereits zu aktiven Zeiten ein Mythos bzw. eine klare Referenz ist (10,5\% aller Trikots mit dem Namen Ronaldinhos und immer noch 5,2\% mit dem Ronaldos - bzw. 28\% und 14\%, wenn man nur die mit einem Namenszug versehenen Trikots einrechnet).

xv Das ,isoliert“ steht bewusst in Anführungszeichen, da die Idee von geschlossenen Kommunikationsgemeinschaften heutzutage getrost als überholt betrachtet werden darf. Die Abgrenzung beziehe ich hier also nicht auf eine fehlende Durchlässigkeit, sondern schlicht auf einzelne Sprachen.

xvi Diese glücklichen (im Falle der WM 1954 und 1978 für Deutschland bzw. Argentinien) oder tief dramatisch-traumatischen (im Falle des für Brasilien zuhause verlorenen Finales bei der WM 1950) Ereignisse sind immer wieder Ausgangspunkt für (meist aktuelle) Überlegungen über Fußball. Dies macht ihre Verankerung im „kollektiven Gedächtnis“ einer Kommunikationsgemeinschaft einmal mehr deutlich.

xvii Die genannten Personen sind - wider Erwarten? - nicht nur im deutschen Fußballdiskurs präsent, sondern ganz wesentlich auch im südamerikanischen: Gerd Müller als Torschützenkönig, Franz Beckenbauer als Spieler bei der WM 1974 fast noch mehr als als Trainer bei der WM 1990 und Lothar Matthäus als Weltfußballer 1991 und (in weitgehend unrühmlicher Rolle) als Trainer in den verschiedensten Ecken der Welt.

xviii In meiner Magisterarbeit diskutiere ich die Möglichkeit, die nicht immer ganz scharfe Trennung zwischen ,Stereotypen' und ,kulturellen Deutungsmustern' zu nutzen, indem ausgehend von Klischees oder Vorurteilen die Meinung von Lernern hinterfragt und - im Sinne eines, kulturellen Lernens ${ }^{\star}-$ mit kontrastierenden Texten konfrontiert wird. 



\title{
„Der Finger in der Wunde“ -
}

\author{
oder einige Anmerkungen zu neurowissenschaftlicher Empirie als \\ Basis didaktisch-methodischer Entscheidungen beim FSL
}

\section{Daniela Zahn (Glasgow)}

Die FLF (Fremdsprachenlernforschung) bezieht sich seit circa 10 Jahren im Zusammenhang ihres Interesses an der Entwicklung einer soliden und kohärenten Fremdsprachenlerntheorie auf Ergebnisse der neurowissenschaftlichen Sprachlernforschung, um didaktisch-methodisches Vorgehen empirisch zu untermauern. Die Übertragung der Ergebnisse der Gehirnforschung auf unterrichtliches Handeln gestaltet sich jedoch schwierig. Das liegt daran, dass die beiden Disziplinen auf unterschiedliche Grundlagenkenntnisse referieren und unterschiedliche Sachgegenstände haben Dass die Übertragung neurowissenschaftlicher Empirie auf didaktischmethodische Handeln unter bestimmten Voraussetzungen gleichwohl möglich sein kann, wird im folgenden am Beispiel prosodischer L2 Forschung zu zeigen versucht.

\section{Einleitung}

Beginnen wir unsere Betrachtungen mit einem kurzen Ausschnitt aus der Geschichte „Kannitverstan“ von Johann Peter Hebeli:

[A]uf dem seltsamsten Umwege kam ein deutscher Handwerksbursche in Amsterdam durch den Irrtum zur Wahrheit und zu ihrer Erkenntnis.... Lange betrachtete er mit Verwunderung dies kostbare Gebäude, die sechs Schornsteine auf dem Dache, die schönen Gesimse und die hohen Fenster, [...]. Endlich konnte er sich nicht enthalten, einen Vorübergehenden anzureden. „Guter Freund“, redete er ihn an, „könnt Ihr mir nicht sagen, wie der Herr heißt, dem dieses wunderschöne Haus gehört ...?“

Der Mann aber, der vermutlich etwas Wichtigeres zu tun batte und zum Unglïck gerade so viel von der deutschen Sprache verstand wie der Fragende von der holländischen, nämlich nichts, sagte kurz und schnaurig: „Kannitverstan!“" und schnurrte vorüber. Dies war nun ein holländisches Wort oder drei, wenn man's recht betrachtet, und heißBt auf deutsch so viel wie: ich kann Euch nicht verstehen. Aber der gute Fremdling glaubte, es sei der Name des Mannes, nach dem er gefragt hatte. „Das muss ein grundreicher Mann sein, der Herr Kannitverstan", dachte er und ging weiter [...]." 
Dieser kurze Ausschnitt aus der Geschichte von Hebel verdeutlicht, neben interkulturellen Differenzen, vor allem eine der Schwierigkeiten, vor der Lerner/innen einer fremden Sprache stehen: wenn eine Person eine ihr begegnende Fremdsprache nicht spricht, kann sie den Lautstrom dieser Sprache nicht ohne weiteres verstehen, weiß also nicht, wann ein Satz, eine Äußerungseinheit, ein Wort beginnt oder endet. Möchte sie diese Sprache lernen, muss sie sich erst in die Sprache hinein hören, um die einzelnen Elemente der Sprache verstehen und lernen zu können. Es stellen sich die Fragen:

Wie funktioniert dieses Hineinhören? Was passiert im Kopf, im Gehirn, wenn ein Mensch sich in etwas hineinhört? Wie könnte dieser Vorgang untersucht werden und, schließlich und endlich, wichtig für die Fremdsprachenvermittlung: Kann dieser Vorgang in irgendeiner Weise unterstützt und erleichtert werden?

Die Neurowissenschaft, oft auch nur Gehirnforschung genannt, versucht die Prozesse und Bedingungen des Sprachenlernens mit ihren eigenen Methoden zu ergründen. Inzwischen gibt es eine kaum noch zu überblickende Anzahl an

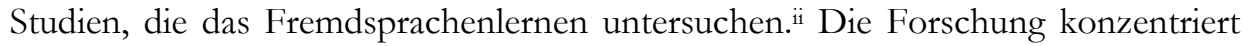
sich hauptsächlich auf folgende Punkte (vgl. Gullberg \& Indefrey 2006):

- proficiency (Niveau oder Sprachkönnensstand)

- convergence (Annäherung der Verarbeitungsprozesse von L1 und L2)

- degeneracy (Verbindung zwischen beobachtbarem Verhalten und zugrunde liegenden Prozessen)

- control (Interaktion zwischen L1 und L2)

Diese Themen werden mit Hilfe bildgebender (funktionale Magnetresonanztomographie, Positronenemissionstomographie) oder elektrophysiologischer (Ereigniskorrelierte Potentiale, Magnetencephalogramm) Verfahren untersucht. Die Ergebnisse der neurowissenschaftlichen L2-Forschung geben Anlass zu der Vermutung, dass es zwischen L1- und L2-Lernen sowohl Gemeinsamkeiten als auch Unterschiede gibt. Es ist aber gleichwohl noch nicht klar, woher die Gemeinsamkeiten und Unterschiede rühren und wie sie zu interpretieren sind. Ein Großteil der Interpretationsunsicherheit ist zurückzuführen auf die verwendeten neurowissenschaftlichen Methoden. Deshalb muss gefragt werden, wie genau L2-Verarbeitungsstudien aufgebaut sind, die diese Methoden verwenden? Und, welche Grenzen sind diesen Methoden gesetzt, die möglicherweise relevant für die Übertragung der Forschungsergebnisse auf die Didaktik / Methodik der Fremdsprachenvermittlung sind? Ich werde mich im Folgenden aus Gründen der Nachvollziehbarkeit auf die Methode der EKP, also der ereigniskorrelierten Potentiale beschränken. 


\section{Ereigniskorrelierte Potentiale}

EKP oder ereigniskorrelierte Potentiale ist eine Methode, bei der Gehirnströme durch Elektroden am Kopf von Versuchspersonen abgeleitet werden, während sie an einem experimentalpsychologischen Versuch teilnehmen. Nach der Auswertung der Daten erhält man ein typisches Kurvenmuster, eine so genannte Komponente. Für die L1-Verarbeitung hat Angela Friederici (Friederici \& Kotz 2003) ein Modell vorgelegt, dem vier Komponenten zugrunde liegen:

- ELAN (early left anterior negativity): tritt auf bei Phrasenstrukturverletzungen

- P600 (Positivierung um 600ms): tritt auf bei grammatischer Reanalyse bzw. Integration

- N400 (Negativierung um 400ms): tritt auf bei semantischen Verletzungen

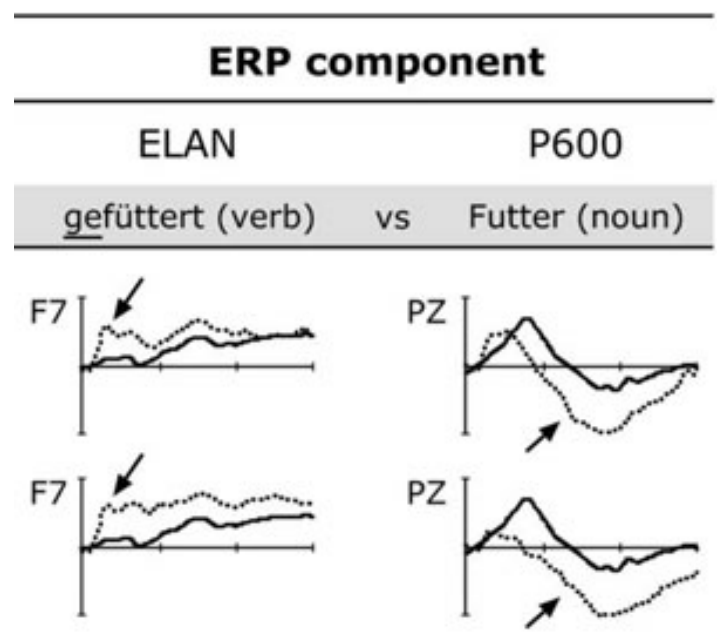

Abb. 1: Analysen von Friederici \& Kotz 2003 (Ausschnitt).

Sprachverarbeitung funktioniert laut diesem Modell wie folgt:

Language comprehension can be subdivided into three processing steps: initial structure building, semantic integration, and late syntactic integration. The two syntactic processing phases are correlated with two distinct components in the event-related brain potential, namely an early left anterior negativity (ELAN) and a late centroparietal positivity (P600). Moreover, ERP findings from healthy adults suggest that early structure-building processes as reflected by the ELAN are independent of semantic processes (Friederici \& Kotz 2003: 58). 
Das heißt, in der Muttersprache wird zuerst eine Phrasenstruktur des Satzes im Gehirn erstellt: Die Kinder essen den Kuchen. [Determiner, Nomen, Verb, Determiner, Nomen]. In diese syntaktische Struktur wird in einem zweiten Schritt die Semantik integriert. Im letzten Schritt wird reanalysiert und die syntaktische Korrektheit sichergestellt. Sprachverarbeitung, das soll an dieser Stelle ausdrücklich betont werden, ist nach Friederici also hauptsächlich syntaxgesteuert. Dieses Modell ist ein sogenanntes syntax-first model.

Das hier skizzierte Modell wird als Vergleichsgrundlage von L1 und L2-Verarbeitung herangezogen. In einer ihrer Studien resümieren Anja Hahne und Angela Friederici ihre Ergebnisse wie folgt:

When comparing the L2-learners brain responses to does of native speakers, a variety of differences were observed. Whereas semantically incorrect sentences showed an ERP pattern similar to that of native listeners (a centro-parietal N 400 effect), correct sentences elicited a greater positivity in L2-learners than in native listeners, possibly in reflection of greater difficulties in syntactic integration. For sentences containing a phrase-structure-violation, L2-learners did not show significant modulations of the syntax-related ERP-components usually seen in native listeners (Hahne \& Friederici 2001: 123).

Die Autorinnen folgern aus ihren Ergebnissen: Die syntaktische Verarbeitung von L2-Lerner/innen unterscheidet sich deutlich von L1-Muttersprachler/inne/n. Aufgrund des geringen Automatisierungsgrads können Phrasenstrukturverletzungen nicht erkannt werden, weshalb späte L2-Lerner/innen auf einer frühen Lernstufe zusätzliche Prozesse auf der Basis konzeptioneller Verarbeitung aktivieren könnten. Lexikalischer Inhalt könnte unabhängig von der morphologischen Struktur des Elements identifiziert werden und könnte dadurch das entsprechende Konzept direkt aktivieren. (vgl. ebd. 137-139; Übersetzung D.Z.)

Wollte man eine Schlussfolgerung für unser Fach aus diesen Ergebnissen ziehen, dann wäre dies folgende: Die Syntax müsste das Primat der Vermittlung sein, wenn muttersprachenähnliche Kompetenz der Lerner/inne/n erreicht werden soll, obwohl auf den unteren Niveaustufen das Lernen lexikalisch erfolgt. Die skizzierten Schlussfolgerungen aus der Beispielstudie sind indes vorschnell und so nicht aus den Untersuchungen abzuleiten. Warum? Dazu sollten wir uns die Untersuchung von Hahne \& Friederici (2001) noch einmal genauer ansehen: Die Probandengruppe bestand aus japanischen Deutschlerner/inne/n. Die Autorinnen machen leider keine Angaben dazu, wie lange die Gruppe schon Deutsch gelernt hat, welche Lehrmaterialen dabei verwendet wurden und welche methodischen Arrangements zum Einsatz kamen. Gesagt wird, dass alle nach dem Alter von 16 Jahren mit dem Lernen begonnen haben. Den Proband/inn/en wurden Sätze als Stimuli auditorisch präsentiert. Die Sätze waren entweder korrekt oder wiesen die folgenden Verletzungen auf: 
- Der Vulkan wurde gegessen. - semantisch, das Zielwort (target) ist hier gegessen

- Das Eis wurde im- gegessen. - syntaktisch, Phrasenstruktur, Präposition verlangt $\mathrm{N}$ nicht $\mathrm{V}$.

- Das Türschloss wurde im- gegessen. - kombiniert semantisch/syntaktisch.

Dazu ist folgendes anzumerken: Bei den Sätzen handelt es sich um Passivsätze. Das Passiv im Deutschen wird normalerweise verwendet, wenn das Agens einer Handlung nicht benannt werden kann oder soll, wenn ein Geschehen besonders betont wird oder bei bestimmten Formen der Aufforderung (Anordung, Ermahnung) (vgl. Rug \& Tomaszewsi 2006; Buscha et al. 1998). Die Funktion des Passiv ist somit recht spezifisch und kontextuell gebunden. Deshalb könnte es sich als problematisch erweisen, wenn passivische Sätze in einem Rezeptionsexperiment in keinen kommunikativen Kontext eingebettet sind. Des Weiteren ist die Reihenfolge der Präsentation der Sätze zufällig, d.h. die Sätze werden einzeln verarbeitet und nicht in einer kommunikativ sinnvollen Situation. Es handelt sich gewissermaßen um eine abstrakt-monologische statt um eine konkret-dialogische Situation. Es stellt sich also die Frage, wie vergleichbar die Verarbeitung von Stimulusmaterial mit der Verarbeitung von realer Kommunikation ist.

EKP-Experimente haben einen sehr restriktiven Anspruch an ihr Stimulusmaterial, es soll ,[...] kurz-dauernd, abrupt einsetzend und endend sowie physikalisch definiert [sein]“" Walesch \& Deuschl 1997: 179). Sprachstimuli sind nie nur von kurzer Dauer, auch wenn ein einzelnes Wort, wie hier gegessen als target angesehen wird. Da sprachliche Äußerungen in einen Kontext eingebettet werden müssen um verstanden zu werden, setzen sie in der Regel nicht abrupt ein - auch wenn hier versucht wurde, durch die Endstellung des Verbs der Anforderung gerecht zu werden. Zudem ist Sprache nicht rein physikalisch definiert, sondern trägt immer Inhalt, was uns zum nächsten Punkt bringt: Wie wird Sprache in diesen Experimenten aufgefasst?

Die Art und Weise, wie die Fehler (Verletzungen) im Einzelnen definiert sind (z. B. als Phrasenstruktur), legt nahe, dass die Auffassung von Sprache, die hier vertreten wird, eher als strukturell - generativistisch verstanden werden kann. Generativistische Sprachbetrachtung geht davon aus, dass Sätze von einer syntaktischen Tiefen- in eine syntaktische Oberflächenstruktur umgewandelt werden. Auf der Oberflächenstruktur erfolgt die Integration von Semantik und Phonologie. Das klingt nicht zufällig bekannt. Hier offenbart sich die Nähe zur Transformationsgrammatik. Aufgrund dieser, bei den Autorinnen nur implizit anklingenden Nähe, gehen Friederici \& Hahne davon aus, dass sich die einzelnen sprachlichen Ebenen voneinander trennen lassen, d.h. dass Semantik, Syntax etc. isoliert untersucht werden können. Natürlich ist diese Trennung ein nicht zu unterschätzender experimenteller Vorteil, lassen sich doch dadurch Stimuli sehr genau definieren.

Schwierig ist das aber für das Fach Deutsch als Fremdsprache, hat sich doch in den letzten Jahrzehnten ein funktionalistischer Blick auf Sprache als fruchtbar 
erwiesen.iii Zumindest die syntaktische Verletzung könnten funktional auch so charakterisiert ${ }^{\mathrm{iv}}$ werden:

Im Satz „Das Eis wurde im- gegessen.“ könnte die Präpositionalphrase sowohl eine unvollständige Modalangabe sein (im Zorn, Affekt ...) als auch eine Ortsangabe (im Kino). In jedem Fall bahnt ,im“ ein neutrales oder maskulines Nominal im Dativ, also eine Präpositionalphrase - verletzt wird also keine Wortkategorie (Partizip statt Nomen, so jedenfalls argumentieren Friederici \& Hahne (2001), setzen also „im gegessen“ statt „im Kino“ als Verletzungsebene an). Vielmehr wird ein unvollständiger Konstituent identifiziert. Auch das ist eine morphosyntaktische Verletzung, weil „im“ morphologische Markierungen impliziert und Kasus, Numerus und Genus der weiteren Präpositionalphrasen-Realisierung vorbahnt und prädeterminiert (zitiert aus E-Mails von Hans Barkowski vom 15. und 16.05.07).

$\mathrm{Zu}$ fragen ist, ob die semantisch distinkte Qualität der Präpositionalphrase Auswirkungen auf die Verarbeitung hat oder nicht. Der funktionalistische Blick auf Sprache legt nahe, dass Form und Bedeutung in direktem Zusammenhang stehen. Es wäre also gelinde gesagt schwierig, bei dieser Herangehensweise, Verletzungen als eindeutig syntaktisch zu beschreiben. Es fehlt an diesem Punkt ein tertium comparationis, auf dessen Basis direkte Schlussfolgerungen aus den neurowissenschaftlichen Daten auf methodisches Handeln im Unterricht erlaubt sind.

Mit Hilfe der EKP wird nicht jede Hirnaktivität gemessen, sondern fast nur die Aktivität einer größeren Anzahl von gleichzeitig feuernden Neuronen des Kortex. Auch ist die Amplitude der Hirnströme sehr gering und wird leicht durch die durch Muskelaktivität entstehende Amplitude überlagert. Deshalb sind beispielsweise Produktionsexperimente nur mit starken Einschränkungen möglich. Dennoch ist die EKP Methode ein etabliertes Verfahren, da replizierbare und reliable Forschungsergebnisse berichtet werden. Es muss jedoch erneut diskutiert werden, wie diese Ergebnisse zu interpretieren sind.

Ein Aspekt der hier skizzierten Studien ist bisher noch nicht angesprochen worden: mit welchen Methoden ist der Sprachstand von den Proband/inn/en erreicht worden? Haben unterschiedliche unterrichtliche Arrangements Einfluss auf den Lernerfolg und ist dieser Einfluss durch neurowissenschaftliche Forschung nachvollziehbar? Diese für uns so wichtigen Fragen werden bisher weniger untersucht. Deshalb könnte man mutmaßen, dass das ungesteuerte Fremdsprachenlernen der Forschungsgegenstand der neurowissenschaftlichen L2-Forschung ist und dem entsprechend für diesen Lernzusammenhang Ergebnisse liefert. Müsste deshalb nicht den verwendeten Unterrichts- und Lehrmethoden verstärkt Aufmerksamkeit geschenkt werden?

Hier könnte eine Brücke geschlagen werden, die sowohl der L2-Lernforschung hilft als auch der neurowissenschaftlichen Forschung neue Perspektiven bietet, nämlich Fragestellungen, die auf didaktisch-methodischem Erfahrungswissen basieren, neurowissenschaftlich zu untersuchen. 


\section{Prosodisches L2-Lernen neurowissenschaftlich untersucht}

Schlussfolgern wir aus den vorangegangenen Ausführungen, welche Anforderungen wir an ein neurowissenschaftliches Experiment haben, das Hinweise auf didaktisch/methodisches Vorgehen liefern kann:

1. Untersucht werden soll das "Wie?" des Lernens, d.h. ein bestimmtes methodisches Vorgehen und dessen Auswirkungen auf den Lernerfolg.

2. Das Vorgehen sollte dazu geeignet sein, sowohl den Anforderungen eines EKPExperiments zu genügen als auch zumindest prinzipiell den Ansprüchen einer funktionalistischen (situativen und interaktiven) Sprachbetrachtung gerecht zu werden.

Verschiedene Gründe sprechen für die Untersuchung eines musikalischprosodischen Trainings zu Beginn des Fremdsprachenlernens.

\section{Zu 1.: Warum bietet sich ein prosodisch-musikalisches Training für eine Untersuchung an?}

Eine Grundvoraussetzung für den Erfolg besonders zu Beginn des Fremdsprachenlernens ist das Dekodieren des fremdsprachlichen Lautstroms. Besonders wichtig ist dabei das Erkennen von Wortgrenzen und Phrasengrenzen sowie das Erkennen des Melodieverlaufs und des Satzakzentes. Diese Merkmale werden prosodisch parametrisiert. Gleichzeitig verdeutlichen sie die Struktur einer Aussage sowohl syntaktisch, indem die einzelnen syntaktischen Komponenten durch Phrasengrenzen voneinander getrennt werden, als auch semantisch, indem die Informationsstruktur der Aussage gekennzeichnet und die emotionale Stimmung des Sprechers/der Sprecherin angezeigt wird. Prosodie erfüllt also Aufgaben, die für das diskursive Sprachverstehen fundamental sind. Deshalb wird Prosodie im Fremdsprachenunterricht wieder verstärkt Bedeutung beigemessen. Dorothy Chun fasst in ihrem Handbuch die Renaissance prosodischen Lernens zusammen:

[...] prior to the 1980 's, most theoretical studies on intonation had not incorporated the changing emphasis on linguistic paradigms into their work. Thus, [...] it was not until the 1980's that the role of intonation in discourse and conversation analysis emerged as a research topic [...]. [T] he FL community, at least in the US, has been moving towards proficiency-oriented methodologies and meaning- and comprehension-based approaches but has only recently begun to recognize intonation as an important component of linguistic and communicative competence (Chun 2002: 109).

Sie folgert daraus für die Unterrichtspraxis:

However, [...] instruction proceeds systematically from word and phrase level to the level of sentence and discourse and begins with awareness training before production (...). At all levels, it is important to include perception/listening exercises as well as production/speaking activities activities (ebd.: 144). 
Das zeigen die neuesten Lehrwerke für Deutsch als Fremdsprache - allerdings fast immer auf Seiten der Produktion, nicht jedoch der Rezeption. Gleichwohl gibt es methodische Ansätze, die ein prosodisches Training als Voraussetzung für Sprachverstehen erfolgreich anwenden, allen voran die Ansätze von Ilse Chauneau (2002) und Sandra Kömer (www.aussprachetraining.de ).

Das intonatorische Training von Krömer beispielsweise basiert auf musikalischen Elementen (Blas- und Schlaginstrumente), die den Rhythmus und die Melodie der deutschen Sprache verdeutlichen. Wie sinnvoll ein solches Vorgehen tatsächlich ist, zeigt die neurowissenschaftliche Forschung: Musik und Sprache teilen die Parameter Rhythmus und Melodieverlauf, d.h. beide haben eine Syntax und auch eine Semantik. Stefan Koelsch und sein Team (2004) konnten in einem priming-Experiment feststellen, dass Sätze und Musikstücke, die ein bestimmtes Zielwort nicht bahnen (to prime), ähnliche N400 Ergebnisse erzielten. In einer weiteren Untersuchung (Koelsch et al. 2005) zeigte sich, dass sich die gleichzeitige Verarbeitung von syntaktisch inkorrekten Wörtern und Akkorden auf die mit der Verarbeitung von Morphosyntax in Verbindung gebrachte LAN (left anterior negativity) qualitativ auswirkt. Daraus zogen die Autor/inn/en den Schluss, dass musikalische und linguistische Syntaxverarbeitung eng zusammenhängen. Auch andere Studien liefern Hinweise für die Annahme, dass ein musikalisches Training den Prosodieerwerb unterstützt, so z.B. Schoen; Magne \& Besson (2004) und Thompson; Schellenberg \& Husain (2004). Einschränkend sei jedoch angemerkt, dass sich der Großteil der hier aufgeführten Studien mit prosodischen Phänomenen auf der Wort- und Satzebene befasst, also wieder nur sehr eingeschränkt auf die Diskursebene anzuwenden ist. Ein prosodisch-musikalisches Training bietet sich also zur Untersuchung an, da die Musik die Brücke zur prosodischen Grundstruktur einer Sprache baut und diese klanglich erfahrbar macht.

\section{Zu 2.: Wie ließe sich ein prosodisch-musikalisches Training neurowissenschaftlich untersuchen?}

Die EKP-Forschung hat sich dem Thema Prosodie mit unterschiedlichen Herangehensweisen angenommen. Zum einen zeigen Korinna Eckstein und Angela Friederici (2005, 2006) mit allen, auch für die vorab besprochenen EKP-Experimente zutreffenden Einschränkungen, dass prosodische Parameter die Verarbeitung von Sprache als Muttersprache auf mindestens zwei syntaktischen Verarbeitungsstufen (ELAN, P600) beeinflussen. Dennoch sind ihre Ergebnisse nicht eindeutig. Pannekamp; Toepel; Alter; Hahne \& Friederici (2005) haben nur eine mit Prosodie verbundene Komponente, das CPS (closure positive shift) ausgemacht, die, je nach experimentellem Design, Phrasengrenzen (Eckstein \& Friederici 2005, 2006) oder die durch Akzente bestimmte Informationsstruktur von Äußerungen (Hruska \& Alter 2004) anzeigt.

Als prosodische Pionierarbeit kann an dieser Stelle die Forschung von Jackson Gandour und seinem Team bezeichnet werden. Er erforscht den Einfluss von Spracherfahrung (language experience) auf die Wahrnehmung prosodischer Muster von englisch/chinesischen Bilingualenv. So konnte beispielsweise gezeigt werden, 
dass die Wahrnehmung von lexikalischen Tönen durch chinesische und englische Proband/inn/en von deren Muttersprache abhängig ist (vgl. Xu et al. 2006, Chandrasekaran 2006). Allerdings muss auch für diese Studien festgehalten werden, dass sie sich mit Phänomenen auf der Wort- und Satzebene beschäftigen und damit nur eingeschränkt den diskursiven Charakter von Sprache untersuchen.

Zusammenfassend gilt für den Bereich der prosodisch-neurowissenschaftlichen L2 Forschung leider: Ergebnisse gibt es wenige und diese sind heterogen. Besser sieht die psycholinguistische Forschungslage aus: Stellvertretend für eine Anzahl von Studien seien die Arbeiten von Slevc \& Miyake (2006) und Tanaka \& Nakamura (2005) genannt. Beiden Arbeiten ist gemeinsam, dass sie einen signifikanten statistischen Zusammenhang zwischen Musikalität und Sprachkönnen nachweisen.

\section{Ein vorläufiges Forschungsdesign}

Eine Studie müsste sich demzufolge diese Forschungsfrage zu Grunde legen: Befördert ein prosodisch-musikalisches Training die Verarbeitung einer unbekannten prosodischen Struktur?

Zur Bearbeitung der Frage eignete sich ein Vorgehen wie folgt: Grundsätzlich muss es sich um ein Pre-Post- Trainingsdesign handeln, das longitudinal angelegt ist ${ }^{\text {vi }}$ Zwei Gruppen von Nullanfängern einer Sprache müssen verglichen werden. Die zu lernende Sprache weist deutliche Unterschiede in der prosodischen Struktur auf (L1 Englisch vs. L2 Italienisch). Die Versuchsgruppe erhält Training, das musikalische Elemente in den Unterricht einbindet und auf Bewusstmachung der zu lernenden L2 Prosodie abzielt. Das Training konzentriert sich auf die prosodischen Parameter Phrasengrenzen (boundaries), Melodieverlauf und Akzente. Die Kontrollgruppe erhält kommunikativ orientierten Fremdsprachenunterricht, allerdings werden keine Elemente eines prosodisch-musikalischen Trainings eingebunden. Der Sprachunterricht darf nicht im Land der Zielsprache stattfinden, um den Einfluss der zielsprachlichen Umgebung so gering wie möglich zu halten.

Im Anschluss an die ersten 15 Stunden werden zum ersten Mal Daten erhoben: behaviorale, d.h.Verhaltensdaten wie Reaktionszeit und Korrektheit der Antworten sowie und EKP-Daten erhoben. Um dem Anspruch auf diskursiv-funktionale Sprachbetrachtung gerecht zu werden, besteht das Stimulusmaterial aus Minidialogen, d.h. einfache Fragen mit einfachen Antworten, die sich daran orientieren, was die Versuchsgruppen im Unterricht und im Training gelernt haben. Den Proband/inn/en werden in einem ersten Experiment gefilterte Minidialogevii auditorisch präsentiert, d.h. auf ihre prosodischen Parameter reduziert. Das Material enthält Dialoge mit bekannten prosodischen Mustern der L1, L2 und prosodischen Mustern einer dritten unbekannten und in ihren Merkmalen distinkten Sprache. Sie lösen eine recognition memory-Aufgabe, d.h. sie müssen entscheiden, ob das präsentierte Muster bekannt oder unbekannt ist. Zu erwarten wäre ein CPS nach 
dem Hauptakzent bei bekannten prosodischen Strukturen.(vgl. Hruska \& Alter 2004), was auf das richtige Dekodieren des präsentierten Materials hinweist. Die Versuchsgruppe müsste bessere Ergenisse aufweisen, jedoch nicht unbedingt im Bereich der Verhaltensdaten. Die EKP-Muster sollten ausgeprägter sein, was für eine tiefere Verarbeitung spräche.

In einem zweiten Experiment erfolgt eine situative Einbettung durch Minidialoge basierend auf Hruska \& Alter (2002). Die Dialoge orientieren sich wieder an dem Kenntnisstand der Probant/inn/en.
Fokus auf neuer Information (broad focus)
Anke arbeitet in Weimar.
Wo arbeitet sie?
[Sie arbeitet in WEImar] [und wohnt in JEna]
Fokus auf kontrastiver Information (narrow focus)
Anke arbeitet in Weimar und wohnt in Jena.
Wohnt sie in Weimar?
[Sie ARbeitet in Weimar] [und WOhnt in Jena.]

Die Verletzungen ergeben sich aus einer zum vorhergehenden Kontext nicht passenden Antwort. Es werden also Dialoge erstellt, in denen die Frage (Wo...?) einen broad focus in der Antwort erwarten lassen müsste. Es folgt aber ein narrow focus, d.h. die Antwort entspricht nicht der zuvor gebahnten. Beispiel:
Anke arbeitet in Weimar.
Wo arbeitet sie?
[Sie ARbeitet in Weimar] [und WOhnt in Jena.]

Die Proband/inn/en lösen eine prosodic judgement-Aufgabe, d.h. sie beurteilen die prosodische Angemessenheit (angemessen oder nicht) der Antwort auf die vorangegangene Frage. Zu erwarten wäre in diesem Dialogkontext bei inkorrekten Sätzen eine N400 gefolgt von einer P600. Dieses Muster würde darauf hindeuten, dass die nicht angemessene Antwort identifiziert wurde (N400), der Fehler als schwerwiegend eingestuft wurde und ein Reanalyseversuch (P600) unternommen wird.

Das zweite Verletzungsmuster würde eine Satzfrage (Wohnt ...?) als Ausgangspunkt haben, der eine broad focus Antwort folgt. Beispiel:

Anke arbeitet in Weimar.

Wohnt Anke in Weimar?

[Sie arbeitet in WEImar] [und wohnt in JEna]. 
Die nicht angemessene Antwort müsste eine N400 auf einem falschen Akzent auslösen. (vgl. Hruska \& Alter 2004). Das würde vermuten lassen, dass zwar die Antwort als nicht angemessen eingestuft wurde (N400), die Verletzung den Verstehensprozess aber nicht so stark beeinflusst, wie das erste Verletzungsmuster. In beiden Konditionen sollte die Versuchsgruppe bessere Verhaltensdaten und ausgeprägtere EKP-Muster zeigen, was auf den Einfluss des Trainings zurückzuführen wäre.

Nach wenigen Stunden Unterricht würde erstmals der Sprachstand der beiden Gruppen erhoben. Die Versuchsgruppe müsste zumindest bei Hörverstehensaufgaben bessere Ergebnisse aufweisen. Die Sprachstandserhebung sollte systematisch wiederholt werden, um zu überprüfen, wie lange ein möglicher Vorteil bestehen bleibt.

\section{Resümee}

Das Lernen einer neuen Sprache stellt große Herausforderungen an unser Gehirn. Ein komplexes linguistisches System muss auf verschiedenen Ebenen (Phonologie, Semantik, Syntax, Pragmatik etc.) erlernt werden. Die Sprachlehr- und -lernforschung stellt sich der Aufgabe, zu ergründen, wie dieses Lernen erleichtert und gefördert werden kann. Dazu ist es notwendig, zu erforschen, was im Gehirn passiert, wenn eine neue Sprache mit verschiedenen Methoden erlernt wird um daraus Schlussfolgerungen für die unterrichtliche Praxis ziehen zu können.

In diesem Artikel wurde am Beispiel des prosodischen L2 Lernens kurz skizziert in welcher Art und Weise neurowissenschaftliche Methoden operationalisiert werden können, damit deren Forschungsergebnisse Ansatzpunkte für didaktischmethodisches Handeln liefern können.

\section{Literatur}

Barkowski, Hans (2002): Das traditionelle Subjekt - eigenständiger Bedeutungsträger in sprachlichen Äußerungen oder abhängiger Aktant von Verben in Prädikatsfunktion? Einige grammatiktheoretische Überlegungen aus fremdsprachenunterrichtlicher Sicht. In: Barkowski, Hans \& Faistauer, Renate (Hrsg.): ... in Sachen Deutsch als Fremdsprache. Sprachpolitik - Unterricht - Interkulturelle Begegnung. Baltmannsweiler: Schneider Verlag Hohengehren, 155-168.

Barkowski, Hans: E-Mails vom 15. und 16.05.2007.

Buscha, Joachim; Freudenberg-Findeisen, Renate; Forstreuter Eike; Koch, Hermann \& Kuntzsch, Lutz (1998): Grammatik in Feldern, Ein Lehr- und Übungsbuch für Fortgeschrittene. Ismaning: Verlag für Deutsch. 
Chandrasekaran Badrinathan; Krishnan, Ananthanarayan \& Gandour Jackson T. (2006): Mismatch negativity to pitch contours is influenced by language experience. In: Brain Research 12(1), 148-56.

Chauneau, Ilse (2002): Hören, Brummen, Sprechen. Handbuch Angewandte Phonetik im Unterrichtsfach Deutsch als Fremdsprache. Stuttgart: Klett.

Chun, Dorothy (2002): Discourse intonation in L2. From theory and research to practice. Amsterdam/Philadelphia: John Benjamin.

Eckstein, Korinna \& Friederici, Angela D. (2005): Late interaction of syntactic and prosodic processes in sentence comprehension as revealed by ERPs. In: Cognitive Brain Research 25(1), 130-43.

Eckstein, Korinna \& Friederici, Angela D. (2006): It's early: ERP-Evidence for Initial Interaction of Syntax and Prosody in Speech Comprehension. In: Journal of Cognitive Neuroscience 18(10), 1696-711.

Davidson, Doug (2006): Strategies for longitudinal neurophysiology, Commentary on Osterhout et al. In: Gullberg Marianne \& Indefrey Peter (Hrsg.): The cognitive neuroscience of second language acquisition. Malden, USA/Oxford, UK : Blackwell, 231-234.

Eisenberg, Peter (2004): Grundriss der deutschen Grammatik. Der Satz. Stuttgart/ Weimar: Metzler.

Friederici, Angela D. \& Kotz, Sonja (2003): The brain basis of syntactic processes. Functional imaging and lesion studies. In: NeuroImage 20, 8-17.

Gullberg, Marianne \& Indefry, Peter (2006): Introduction. In: Dies. (Hrsg.): The cognitive neuroscience of second language acquisition. Malden, USA/Oxford, UK: Blackwell, 1-8.

Hahne, Anja. \& Friederici, Angela D. (2001): Processing a second language: late learners‘ comprehension mechanisms as revealed by event-related brain potentials. In: Bilingualism: Language and Cognition 4(2), 123-141.

Hruska, Claudia \& Alter, Kai (2004): Prosody in dialoges and single sentences: How prosody can influence speech perception. In: Steube, Anita (Hrsg.): Information structure. Theoretical and empirical aspects. Berlin/New York: De Gruyter, 211-226.

Koelsch, Stefan; Gunter, Thomas; Wittfoth, Matthias \& Sammler, Daniela (2005): Interaction between Syntax Processing in Language and in Music: An ERP Study. In: Journal of Cognitive Neuroscience 17(10), 1565-77.

Koelsch, Stefan; Kasper, Elisabeth, Sammler, Daniela, Schulze, Katrin, Gunter, Thomas \& Friederici, Angela D. (2004): Music, language and meaning: brain signatures of semantic processing. In: Nature Neuroscience 7(3), 302-7.

Krömer, Sandra (2001): Musik der Sprache. Rhythmus, Klang, Bewegung - ein neues Kon₹ept zum Erlernen einer Sprache. In: Deutsch als Zweitsprache, Extraheft.

Krumm, Hans-Jürgen (1988): Grammatik im kommunikativen Deutschunterricht, Konsequenzen für eine didaktische Grammatik und für das Lehrverhalten. In: Dahl, Johannes \& Weis, Brigitte (Hrsg.): Handbuch Grammatik im Unterricht. München: Goethe-Institut, 5-44. 
Lamers, Monique (2006): Cracking the nutshell differently. Commentary on Müller. In: Gullberg, Marianne \& Indefrey Peter (Hrsg.): The cognitive neuroscience of second language acquisition. Malden, USA/Oxford, UK: Blackwell, 271-277.

Müller, Jutta (2006): L2 in a nutshell: The investigation of second language processing in the miniature language model. In: Gullberg Marianne \& Indefrey Peter (Hrsg.): The cognitive neuroscience of second language acquisition, Malden, USA/Oxford, UK: Blackwell, 235-270.

Osterhout, Lee; McLaughlin, Judith; Pitkänen, Ilona; Frenck-Mestre, Cheryl \& Molinaro, Nicola (2006): Novice learners, longitudinal designs and event related brain potentials: A means for exploring the neurocognition of second language processing. In: Gullberg Marianne \& Indefrey Peter (Hrsg.): The cognitive neuroscience of second language acquisition, Malden, USA/Oxford, UK: Blackwell, 199-230.

Pannekamp, Anja; Toepel, Ulrike; Alter, Kai; Hahne, Anja. \& Friederici, Angela D. (2005): Prosody-driven sentence processing: an event-related brain potential study. In: Journal of Cognitive Neuroscience 17(3), 407-421.

Pickering, Martin \& Garrod, Simon (2004): Towards a mechanistic psychology of dialogue. In: Behavioral and brain science 27, 1-22.

Rug, Wolfgang \& Tomaszewski, Andreas (2001): Grammatik, mit Sinn und Verstand. Stuttgart: Klett.

Schoen Daniele; Magne, Cyrille \& Besson, Mireille (2004): The music of speech: music training facilitates pitch processing in both music and language. In: Psychophysiology 41(3), 341-349.

Slevc, L. Robert \& Miyake, Akira (2006): Individual Differences in Second-Language Proficiency: Does Musical Ability Matter? In: Psychological Science 17(8), 675-681.

Tanaka, Akihiro \& Nakamura, Kuninori (2005): Auditory memory and proficiency of second language speaking: a latent variable analysis approach. In: Psychological Report 95(3), 723-734.

Toepel, Ulrike \& Alter, Kai (2004): On the independence of information structural processing from prosody. In: Steube, Anita. (Hrsg.): Information structure. Theoretical and empirical aspects. Berlin/New York: De Gruyter, 227-240.

Thompson, William F.; Schellenberg E. Glenn \& Husain, Gabriella (2004): Decoding speech prosody: do music lessons help? In: Emotion 4(1), 46-64.

Wallesch, Claus-Werner \& Deuschl, Günther (1997): Elektrophysiologie am Menschen. In: Kischka, Udo; Wallesch C.W. \& Wolf, Gerald (Hrsg.): Methoden der Hirnforschung. Eine Einführung. Heidelberg/Berlin, 167-185.

Wing, Herbert D. (1968): Tests of musical ability and apprehension: An investigantion into measurement, distribution and development of musical capacity, 2. Aufl., London [u.a.]: Cambridge University Press. 
Xu Yisheng; Gandour Jackson T. \& Francis, Alexander L. (2006): Effects of language experience and stimulus complexity on the categorical perception of pitch direction. In: Journal of the Acoustic Society of America 120(2), 1063-1074.

Zahn, Daniela \& Döpel, Martin (2007): Optimale - Potentiale - Universell - Vernetzt: Ausgewählte kognitions- und sprachwissenschaftliche Aspekte des L2-Lernens. In: Eßer Ruth \& Krumm Hans-Jürgen (Hrsg.): Bausteine für Babylon: Sprache, Kultur, Unterricht. München: Iudicium, 54-69.

i Hebel, J.P. [http://gutenberg.spiegel.de; 07.11.2007].

ii Einen hervorragenden Überblick geben Marianne Gullberg und Peter Indefrey (2006).

iii Ich verweise ausdrücklich auf: Peter Eisenberg (2004) sowie auf Beiträge von Hans Barkowski und Hans-Jürgen Krumm.

iv Ich danke Hans Barkowski für seine sehr hilfreichen Kommentare und Anregungen zu diesem Thema.

v In ihren Veröffentlichungen sprechen Gandour und Kolleg/inn/en immer wieder von Bilingualen als Proband/inn/en. Sie erklären allerdings nicht, was sie unter Bilingualität verstehen.

vi Zu den Vorteilen dieser Vorgehensweise vgl. Osterhout et al. (2006). 




\section{Die Autor/inn/en}

Uta Großmann, M.A.; Doktorandin am Herder-Institut Leipzig; Forschungsinteressen: Vermittlung von methodisch-didaktischem Vorgehen im Unterricht DaF, Leseverstehensprozessforschung, Erforschung der Vermittlung von Wirtschaftssprache in $\mathrm{DaF}$

Barbara Haider, Mag. Dr.phil.; Lektorin an der Universität Wien, Institut für Germanistik/Deutsch als Fremdsprache; DaZ-Trainerin am Jugendbildungszentrum an der VHS Ottakring; gegenwärtige Forschungsthemen und -interessen: Deutsch am Arbeitsplatz, Deutsch für Jugendliche

Cordula Hunold, M.A., Dr.phil.; Wissenschaftliche Mitarbeiterin an der Abteilung Interkulturelle Germanistik der Universität Göttingen; Promotion im Bereich Sprechwissenschaft und Phonetik am Seminar für Sprechwissenschaft und Phonetik der Martin-Luther-Universität Halle/Wittenberg; Forschungsthemen und interessen: Untersuchungen zu Ausspracheproblemen chinesischer Deutschlernender, Interkulturelle Kommunikation Deutschland-China, Chinesisch als Fremdsprache

Isabelle Maringer, M.A.; Wissenschaftliche Mitarbeiterin am Herder-Institut der Universität Leipzig; Koordination des $\mathrm{PhD}$-Promotionsstudienganges Deutsch als Fremdsprache/Transcultural German Studies; Forschungsinteressen: Kulturstudien Deutsch als Fremdsprache, Kulturtheorien, Deutungsmusteranalysen

Kristina Peuschel, M.A.; Wissenschaftliche Mitarbeiterin am Herder-Institut der Universität Leipzig, Promotion im Bereich Didaktik/Methodik zum Spracherwerb in medienspezifischen Projekten; Forschungsinteressen: offene Lernformen, individuelle Schwierigkeiten, Lernberatung und aktive Medienarbeit im Fremdsprachenunterricht

Jan Paul Pietzuch, M.A.; Wissenschaftlicher Mitarbeiter an der Fakultät für Linguistik und Literaturwissenschaft der Universität Bielefeld (DaF/DaZ); Forschungsinteressen: linguistische und sozialwissenschaftliche Diskurstheorie/analyse, Bildungstheorie/-forschung, Auswärtige Kulturpolitik, soziokulturelle Ansätze der Spracherwerbsforschung 
Anja Ucharim, M.A.; Stipendiatin und Doktorandin am Herder-Institut Leipzig, Forschungsthemen und -interessen: Kulturstudien, Literaturwissenschaft, Lehrwerkanalyse, DaZ.

Sabine Christine Stricker, M.A., Lektorin für Deutsch als Fremdsprache an der Universität Trient, Referentin für Lehrerfortbildungen und wissenschaftliche Beraterin des Projektes „L.E.S.I. - Sprachkontakte im Kindergarten“ des Kindergartenverbandes der Provinz Trient. Forschungsschwerpunkte: Sprachenpolitik, innovative Methoden und Ansätze, frühkindliches Fremdsprachenlernen.

Paul Voerkel, M.A.; Studium in DaF, Geschichte und Hispanistik/Spanisch in Leipzig, Warschau und Curitiba; 2007/08 DAAD-Sprachassistent an der UFPA in Belém (Brasilien); Forschungsinteressen und -schwerpunkte: Kulturstudien, Diskursanalyse, angewandte Methodik/Didaktik, Curricula-Entwicklung

Daniela Zahn, M.A.; Postgraduate an der Glasgow University / Department of Psychology; Forschungsthemen und -interessen: Interaktion von Prosodie und Syntax in der Sprachverarbeitung/Sprachproduktion, psycholinguistische/neurolinguistische Forschungsmethoden und deren Übertragbarkeit auf die Sprachlehrund Lernforschung, qualitative Forschungsmethoden in der Psychologie 
Die Beiträge des Sammelbands bieten einen kaleidoskopischen Blick auf innovative Forschungsfragen und -instrumentarien laufender oder abgeschlossener Qualifikationsprojekte im Wissenschaftsbereich Deutsch als Fremd-/Zweitsprache. Unter anderem werden Studien aus der Textverstehensforschung, der critical needs analysis, der sozialwissenschaftlichen Diskursanalyse, der empirischen Didaktik und der kulturbezogenen Lernprozessforschung zur Diskussion gestellt. Dem Prinzip der lebendig-bunten Bilderfolge eines Kaleidoskops entsprechend, verzichten die Herausgeber auf eine fachsystematische Einordnung der Beiträge und erheben keinen Anspruch auf eine ,Kartografie' des derzeitigen Stands junger DaF-/DaZ-Forschung. Vielmehr betont das Bild des Kaleidoskops das Fragmentarische der Dokumentation, ermuntert aber auch dazu, die Funktionsweise eines solchen optischen Spielzeugs als Leseperspektive anzunehmen. Im Sinne des „Schönbildschauers“ (kalós: schön; eĩdos: Gestalt, Bild; skopeĩn: schauen) eröffnet der Band einen optimistischen Blick auf die Entwicklung der jungen DaF-/DaZ-Forschung und regt dazu an, den Dialog über Struktur und Instrumente der DaF-/DaZ-Nachwuchsförderung zu intensivieren.

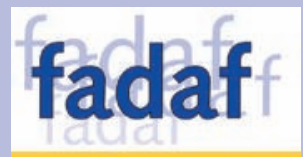

fadaf.de

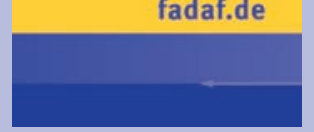

